\title{
Delayed-Enhanced Cardiovascular Magnetic Resonance in the diagnosis and management of Cardiac Sarcoidosis
}

Citation for published version (APA):

Smedema, J-P. (2019). Delayed-Enhanced Cardiovascular Magnetic Resonance in the diagnosis and management of Cardiac Sarcoidosis. [Doctoral Thesis, Maastricht University]. ProefschriftMaken Maastricht. https://doi.org/10.26481/dis.20190704js

Document status and date:

Published: 01/01/2019

DOI:

10.26481/dis.20190704js

Document Version:

Publisher's PDF, also known as Version of record

Please check the document version of this publication:

- A submitted manuscript is the version of the article upon submission and before peer-review. There can be important differences between the submitted version and the official published version of record.

People interested in the research are advised to contact the author for the final version of the publication, or visit the DOI to the publisher's website.

- The final author version and the galley proof are versions of the publication after peer review.

- The final published version features the final layout of the paper including the volume, issue and page numbers.

Link to publication

\footnotetext{
General rights rights.

- You may freely distribute the URL identifying the publication in the public portal. please follow below link for the End User Agreement:

www.umlib.nl/taverne-license

Take down policy

If you believe that this document breaches copyright please contact us at:

repository@maastrichtuniversity.nl

providing details and we will investigate your claim.
}

Copyright and moral rights for the publications made accessible in the public portal are retained by the authors and/or other copyright owners and it is a condition of accessing publications that users recognise and abide by the legal requirements associated with these

- Users may download and print one copy of any publication from the public portal for the purpose of private study or research.

- You may not further distribute the material or use it for any profit-making activity or commercial gain

If the publication is distributed under the terms of Article $25 \mathrm{fa}$ of the Dutch Copyright Act, indicated by the "Taverne" license above, 


\title{
Delayed-Enhanced Cardiovascular Magnetic Resonance in the diagnosis and management of Cardiac Sarcoidosis
}

\author{
Jan-Peter Smedema
}


(C) Jan-Peter Smedema, Cape Town 2019

Printing: ProefschriftMaken || www.proefschriftmaken.nl

ISBN 978-94-6380-419-6

All rights reserved. No part of this publication may be reproduced, stored in a retrieval system or transmitted, in any form or by any means, electronic, mechanical, photocopying, recording or otherwise, without prior permission of the author or the copyright-owning journals for previous published chapters. 


\title{
Delayed-Enhanced Cardiovascular Magnetic Resonance in the diagnosis and management of Cardiac Sarcoidosis
}

\author{
Proefschrift/Dissertation \\ ter verkrijging van de graad van doctor \\ aan de Universiteit Maastricht, \\ op gezag van de Rector Magnificius, Prof. dr. Rianne M. Letschert, \\ volgens het besluit van het college van Decanen, \\ in het openbaar te verdedigen op \\ donderdag 4 juli 2019 om 12.00 uur \\ to obtain the degree of Doctor at the University of Maastricht, \\ under the authority of the Rector Magnificus Prof. dr. Rianne M. Letschert, \\ according to the decision of the College of Deans, \\ to defend in public on Thursday, July 4, 2019 at noon. \\ door/by \\ Jan-Peter Smedema
}




\section{Promotor}

Prof. dr. H.J.G.M. Crijns

\section{Beoordelingscommissie/Assessment Committee}

Prof. dr. S. Heymans (voorzitter/chairman)

Prof. dr. F.E. Rademakers, University Hospitals Leuven, Belgium

Prof. dr. A.C. van Rossum, Amsterdam University Medical Centres

Prof. dr. P.G.A. Volders

Prof. dr. GJ. Wesseling

Financial support by Stichting Hartsvrienden RESCAR of Maastricht is gratefully acknowledged. 


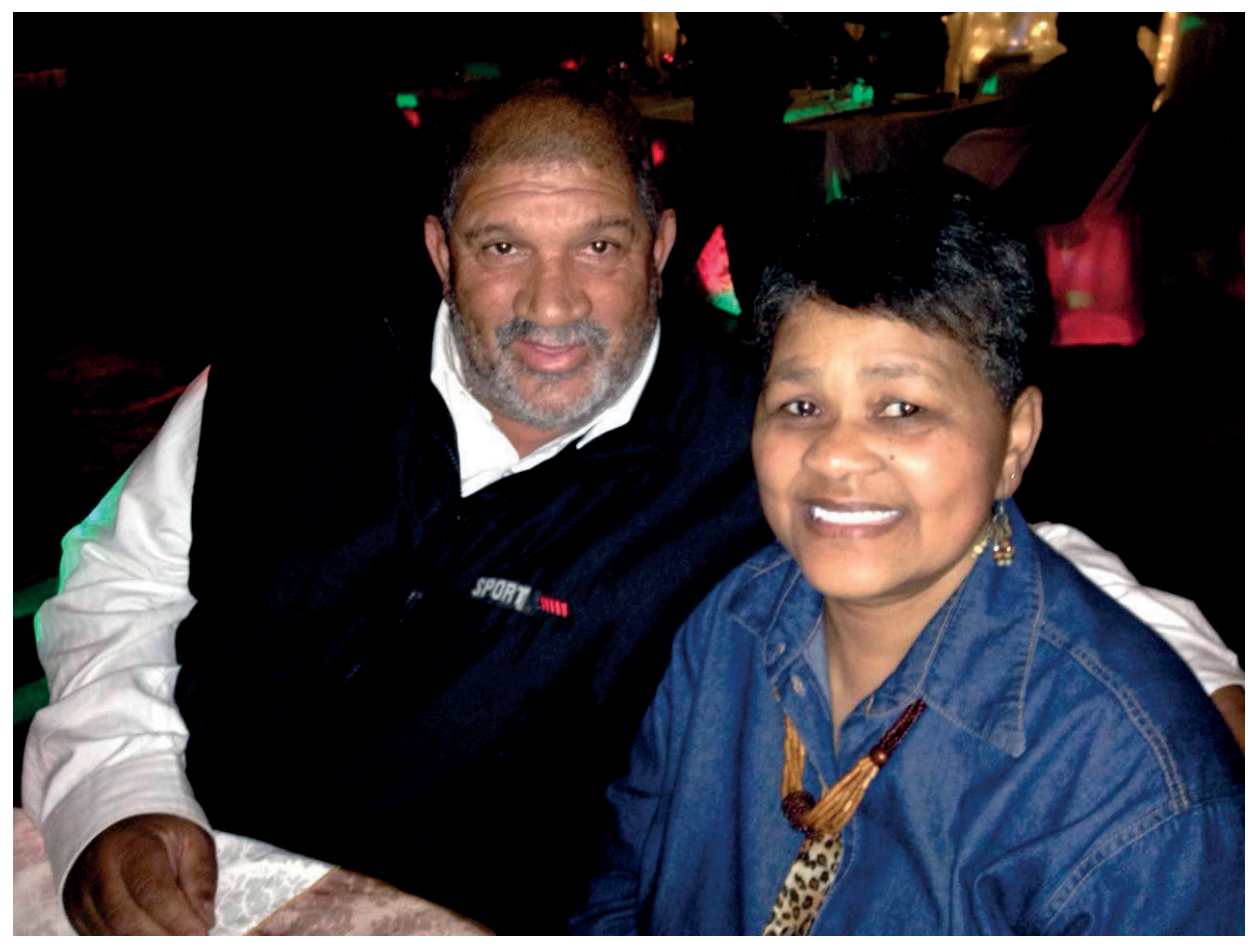

This dissertation is dedicated to mrs Specilene Francis, the first patient with cardiac sarcoidosis I encountered in Cape Town (1998). She presented with sustained monomorphic ventricular tachycardias, which originated from the lateral left ventricle. Delayed contrastenhanced cardiovascular magnetic resonance revealed extensive patchy granulomatous infiltration and focal scar (19\% of left ventricular mass), with involvement of the posterolateral papillary muscle resulting in severe eccentric mitral regurgitation. Her condition has remained stable on maximal medical treatment which initially included immune suppressive agents. A Cardioverter Defibrillator was implanted. After an initial electrical storm, she has remained free from sustained arrhythmias, device therapy and hospital admission. Specilene enjoys an active life with her husband of 37 years. 


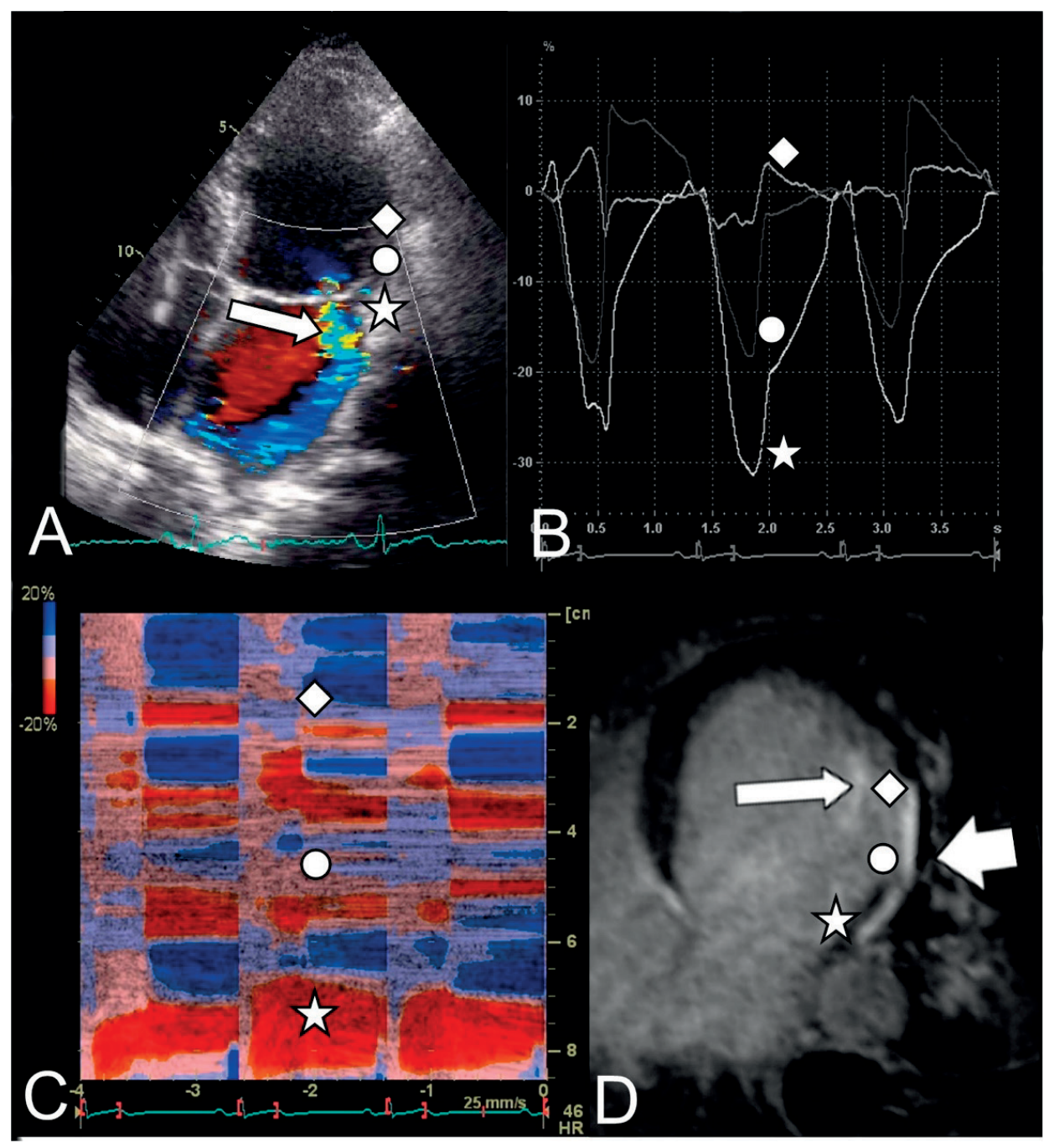

Follow up transthoracic echocardiography in mrs Francis (2005) demonstrated severe eccentric mitral regurgitation (panel A: 4 chamber view, arrow), secondary to papillary muscle dysfunction; tissue Doppler sampling of the longitudinal strain reveals normal deformation at the level of the lateral mitral annulus *, but impaired deformation of the basal ${ }^{\circ}$ and mid lateral ${ }^{\diamond}$ left ventricular segments, (panels $\mathbf{B}$ and $\mathbf{C}$ : respectively graphical and curved linear MMode representation); delayed contrast-enhanced cardiovascular magnetic resonance confirms the presence of extensive scarring of the mentioned segments (panel D: DECMR 4 chamber view, left sided arrow - contrast enhanced papillary muscle, right sided arrow - partly subendocardial, partly transmural lateral wall contrast enhancement i.e. myocardial scar tissue). (Smedema JP. Tissue Doppler Imaging in cardiac sarcoidosis. Eur J Echocard 2008;9:579-580. doi: 10.1093/ejechocard/jen073.) 


\section{Contents}

Chapter 1 Introduction 9

Chapter 2 Review - Contrast-enhanced magnetic resonance in the diagnosis and 11 management of cardiac sarcoidosis

(submitted in abbreviated form)

Chapter 3 Cardiac involvement in patients with pulmonary sarcoidosis assessed at two University Medical Centres in The Netherlands (Smedema JP, Snoep G, van Kroonenburgh MPG, van Geuns, RJ, Dassen WRM, Gorgels AP, Crijns, HJGM. Chest 2005;128(1):30-5. doi: 10.1378/chest.128.1.30.)

Chapter 4 The additional value of gadolinium enhanced magnetic resonance imaging to standard assessment for cardiac involvement in patients with pulmonary sarcoidosis (Smedema JP, Snoep G, van Kroonenburgh MPG, Gorgels APM, Crijns HJGM. Chest 2005;128(3):1623-1628. doi: 10.1378/ chest.128.3.1629.)

Chapter 5 Evaluation of the accuracy of gadolinium-enhanced cardiovascular magnetic resonance in the diagnosis of cardiac sarcoidosis (Smedema JP, Snoep G, van Kroonenburgh MJPG, van Geuns RJ, Dassen WRM, Gorgels AP, Crijns HJGM. J Am Coll Cardiol 2005;45(10):1683-1690. doi: 10.1016/j.jacc.2005.01.047.)

Chapter 6 Contrast-Enhanced Cardiac Magnetic Resonance: Distinction between cardiac sarcoidosis and infarction scar (Smedema JP, van Geuns RJ, Truter R, Mayosi BM, Crijns HJGM. Sarc V asc Diff Lung Dis 2017;34:307-314. http:// wnw.mattioli1885journals.com/index.php/sarcoidosis/article/view/5715.)

Chapter 7 Right ventricular involvement in cardiac sarcoidosis demonstrated with 161 cardiac magnetic resonance

(Smedema JP, van Geuns RJ, Ector J, Heidbuchel H, Ainslie G, Crijns HJGM. ESC Heart Failure 2017;4(4):535-544. doi: 10.1002/ ebf2.12166.) 
Chapter 8 Right ventricular involvement and the extent of left ventricular enhancement with magnetic resonance predict adverse outcome in pulmonary sarcoidosis

(Smedema JP, van Geuns RJ, Ector J, Heidbuchel H, Ainslie G, Crijns HJGM. ESC Heart Failure 2018;5(1):157-171. doi: 10.1002/ ehf2.12201.)

Chapter 9 General discussion

Chapter 10 Summary

Chapter 11 Valorisation

Chapter 12 Acknowledgements

Chapter 13 Curriculum Vitae

235 


\section{Chapter 1}

\section{Introduction}

In 1998 we encountered a 40-year-old housewife in Cape Town who presented with sustained monomorphic ventricular tachycardia, and for the first time included Delayed Contrast-Enhanced Cardiovascular Magnetic Resonance (DECMR) in the diagnostic process. Endomyocardial biopsies confirmed cardiac sarcoidosis (CS). This experience introduced me to the world of cardiovascular magnetic resonance, and its potential to diagnose and help manage this intriguing condition. Apart from several case-reports no systematic research had been performed to determine the role of DECMR in the diagnosis, management and risk assessment of CS. Most experience in the management of CS and relevant published literature on this topic originated in Japan. The only existing Diagnostic Guidelines, which at that stage did not include DECMR, had been compiled in that country. After professor Dudley Pennell offered me accredited CMR training at the Royal Brompton Hospital, I was determined to formally study the role of DECMR in CS. This journey took me from Cape Town, and London, to Rotterdam, Maastricht and eventually back to Cape Town. The diagnostic tools available at that time were resting, exercise and ambulant electrocardiography, 2D echocardiography, as well as gallium, thallium and technetiumMIBI scintigraphy. These modalities were not sensitive or specific for the diagnosis of CS, and detected abnormalities frequently non-specific in nature. The prevalence and extent of CS remained underestimated, and detailed information on the myocardial distribution of inflammation and scarring was not provided. Postmortem studies in the United States suggested that $25-30 \%$ of sarcoidosis patients had either clinical or subclinical cardiac involvement. The prevalence of CS in The Netherlands was unknown. Diagnostic evaluation with the available armamentarium provided a substantially lower prevalence of CS. The first presentation of CS in some patients unfortunately was (aborted) sudden cardiac death. Clinicians needed a safe, accurate, robust and non-invasive diagnostic tool for CS, and DECMR held great promise.

\section{The aim of our research was}

1. To determine the prevalence of cardiac involvement in patients with pulmonary sarcoidosis in The Netherlands.

2. To determine the distribution and extent of myocardial sarcoidosis with DECMR. 
3. To determine the diagnostic accuracy of DECMR for cardiac involvement in patients with pulmonary sarcoidosis compared to the gold-standard diagnostic Japanese Guidelines (1993) at that time (2005).

4. To determine the additional diagnostic value of DECMR for CS compared to the standard cardiac evaluation with resting 12-lead electrocardiography, 24-hour ambulant electrocardiography, transthoracic 2D Doppler echocardiography, and thallium or technetium-MIBI scintigraphy in patients with pulmonary sarcoidosis.

5. To determine the predictive value of DECMR for adverse cardiac outcome in patients with pulmonary sarcoidosis and cardiac involvement.

These research questions shaped the structure of this thesis. Chapter 2 reviews the relevant clinical and pathophysiological background of CS, whilst introducing DECMR and detailing its role in the management of CS. The prevalence of CS, diagnostic accuracy and additional value of DECMR in addition to standard assessment in Dutch patients with pulmonary sarcoidosis at the University Hospitals of Rotterdam and Maastricht are detailed in Chapters 3 and 4. DECMR allows for the detection and quantification of focal scar and interstitial fibrosis. By 2016 numerous reports had been published on DECMR delineating left ventricular sarcoidosis, but relatively few studies reported on right ventricular involvement. Chapter 5 describes the prevalence and distribution of right ventricular late gadolinium enhancement (LGE) in patients diagnosed with pulmonary sarcoidosis, and determines the relationship with pulmonary hypertension, ventricular volumes, mass and systolic function. The non-ischemic pattern of LGE in CS potentially distinguishes patients from those with LGE due to ischemic scar. In Chapter $\mathbf{6}$ we describe the DECMR studies of 30 patients with CS, which were compared to those, performed in 30 consecutive myocardial infarct patients, who had been managed with primary coronary interventions. Blinded observers classified patients as either suffering from CS or ischemic heart disease by assessing the distribution of LGE. The prognostic value of DECMR is discussed in Chapter 7. Chapter 8 puts the results obtained in this thesis in perspective. 
Chapter 2

\section{Review - Contrast-enhanced magnetic} resonance in the diagnosis and management of cardiac sarcoidosis 


\section{Contents}

Abbreviations 13

2.1 Introduction 15

2.2 Histopathology of cardiovascular sarcoidosis $\quad 15$

2.3 Principles of Cardiovascular Magnetic Resonance and the application in cardiac sarcoidosis $\quad 17$

2.4 Contrast agents and their application in the evaluation of cardiac sarcoidosis 19

2.5 Determining focal fibrosis (Tables 4, 5) 20

2.6 The measurement of extracellular volume and diffuse fibrosis with T1 mapping $\quad 21$

2.7 CMR assessment of myocardial inflammation in sarcoidosis 23

2.8 The potential of Ultra Small Super paramagnetic and Nano particles in the assessment of mononuclear myocardial inflammation 24

2.9 Cardiovascular Magnetic Resonance in cardiac sarcoidosis: the first $\begin{array}{ll}\text { publications } & 25\end{array}$

2.10 The CMR diagnosis and differential diagnosis of cardiac sarcoidosis 25

2.11 Isolated cardiac sarcoidosis 26

2.12 The significance of evaluating for mediastinal lymphadenopathy in cardiomyopathy 26

2.13 Role of cardiovascular magnetic resonance in the guidelines and position $\begin{array}{ll}\text { papers } & 27\end{array}$

$\begin{array}{ll}2.14 & \text { Atrial sarcoidosis } \\ 2.15 & 27\end{array}$

2.15 Right ventricular sarcoidosis $\quad 27$

2.16 First-pass myocardial perfusion and perfusion reserve in sarcoidosis 29

2.17 Pericardial sarcoidosis 29

2.18 Macro granulomas 29

2.19 Can the distribution of LGE differentiate between different $\begin{array}{ll}\text { cardiomyopathies? } & 30\end{array}$

2.20 Prognostic value of DECMR - CMR as part of risk stratification 30

2.21 The relevance of screening for subclinical cardiac sarcoidosis 31

2.22 DECMR assists in clinical management of cardiac sarcoidosis 31

2.23 Improved outcomes with modern management $\quad 32$

2.24 Conclusions $\quad 32$

2.25 New and future applications of CMR in the management of cardiac
sarcoidosis (Table 12)

2.26 Acknowledgements 33

References $\quad 34$ 


\section{Abbreviations}

18F-FDG PET = 18F-

Fluorodeoxyglucose Positron Emission Tomography

67-Ga = Gallium-67

201-Th $=$ Thallium-201

$\mathrm{ACE}=$ angiotensin converting enzyme

AIR $=$ Arrhythmia-Insensitive-Rapid

ANGIE = Accelerated and Navigator-

Gated look-locker imaging

$\mathrm{ARCV}=$ arrhythmogenic right ventricular cardiomyopathy

$\mathrm{AVB}=$ atrio-ventricular block

$\mathrm{CID}=$ cardiac implanted devices

$\mathrm{CAD}=$ coronary artery disease

$\mathrm{CMR}=$ cardiovascular magnetic resonance

$\mathrm{CRT}=$ cardiac resynchronization thera-

py

$\mathrm{CS}=$ cardiac sarcoidosis

$\mathrm{CT}=$ computed tomography

$\mathrm{CU}=$ cardiac ultrasound

DECMR = delayed contrast-enhanced

cardiac magnetic resonance

$\mathrm{DC}=$ dendritic cells

$\mathrm{ECG}=$ electrocardiogram

$\mathrm{ECV}=$ extracellular volume fraction

$\mathrm{EMB}=$ endomyocardial biopsies

GBCA $=$ Gadolinium-Based Contrast

Agents

GraSE $=$ Gradient-Spin-Echo

HASTE $=$ Half-Fourier Acquisition

Single-shot Turbo-spin-Echo

HCM = hypertrophic cardiomyopathy

HRS $=$ Heart Rhythm Society

$\mathrm{Ht}=$ hematocrit

ICD = implantable cardioverter-

defibrillator

ICS $=$ isolated cardiac sarcoidosis

IGCM = giant cell myocarditis

IR-GRE = Inversion-Recovery Gradient

Echo sequence
IR-FGRE = Inversion Recovery Fast

Gradient Echo

JMHW = Japanese Ministry of Health and Welfare

LGE = late gadolinium enhancement

$\mathrm{LV}=$ left ventricle

LVEDV = left ventricular end-diastolic volume

LVEF = left ventricular ejection fraction

$\mathrm{LVH}=$ left ventricular hypertrophy

MCLE = Multi-Contrast Late Enhancement Imaging

MDCT $=$ multi-detector computed tomography

MGC $=$ Multi-nucleated Giant Cells

$\mathrm{MFR}=$ myocardial flow reserve

MHC = Major Histocompatibility Complex

MOLLI $=$ MOdified Look-Locker Inversion recovery

MPS $=$ myocardial perfusion scintigraphy

NICM $=$ non-ischemic cardiomyopathies

$\mathrm{NSF}=$ nephrogenic systemic fibrosis

$\mathrm{nsVT}=$ non-sustained ventricular tachycardia

PAH $=$ pulmonary arterial hypertension

PES = programmed electrical stimulation

PET $=$ positron emission tomography

$\mathrm{PH}=$ pulmonary hypertension

$\mathrm{PM}=$ pacemaker

PSIR $=$ Phase-Sensitive Inversion Re-

covery

RFA $=$ radiofrequency ablation

$\mathrm{RV}=$ right ventricle

$\mathrm{RVH}=$ right ventricular hypertrophy

sVT = sustained ventricular tachycardia

SAP-T1 $=$ Short Acquisition Period -

T1 mapping 
SAPPIRE $=$ SAturation Pulse Prepared heart-rate independent Inversion REcovery

SASHA $=$ SAturation recovery SinglesHot Acquisition

SCD $=$ Sudden Cardiac Death

SCMR $=$ Society for Cardiovascular magnetic Resonance

ShMOLLI $=$ Shortened MOdified Look-Locker Inversion recovery

SPECT $=$ Single Photon Emission Computed Tomography

SPIR $=$ spectral presaturation with inversion recovery
STONE $=$ Slice-interleaved T1 mapping SSFP $=$ Steady-State-Free Precession STIR $=$ Short Tau Inversion Recovery TWAaVR $=$ T-wave amplitude in lead aVR

$\mathrm{TE}=$ echo time

$\mathrm{TI}=$ inversion time

$\mathrm{TR}=$ repetition time

$\mathrm{VT}=$ ventricular tachycardia

$\mathrm{VF}=$ ventricular fibrillation

WASOG $=$ World Association for Sarcoidosis and Other Granulomatous Disorders 


\subsection{Introduction}

Sarcoidosis is a rare inflammatory condition, during which the deranged Th1 cellular immune response, triggered by a non-specific non-organic (insecticides, building dust, fumes, cosmetics) or organic particle (molds, tree pollen, viruses, Propionibacterium, Mycobacteria), forms the hallmark non-caseating granulomas and subsequent fibrosis. (1-5) In genetically predisposed individuals this condition can potentially affect any tissue or solid organ. Approximately $20 \%$ of patients suffer from concomitant autoimmune conditions such as inflammatory bowel, thyroid or thymal disease. (1-3,5,7) In $90 \%$ of patients the lungs and mediastinal lymph nodes are predominantly affected, which suggest that exposure to the agent generally occurs by inhalation. $(\mathbf{1 - 3 , 8 )}$ Sarcoidosis originally received its name because of the "flesh like" skin lesions first described by Hutchinson in 1898 which macroscopically resembled "sarcoma". (9) Bernstein et al (1929) were the first to report cardiac involvement in a 52 year-old Caucasian tailor, which resulted in congestive heart failure and death. (10) While $5-10 \%$ of sarcoidosis patients present with clinically manifest cardiac involvement, postmortem and imaging studies have revealed the heart to be affected in 20$30 \%$ of sarcoidosis patients. The basal left ventricular segments and right side of the interventricular septum are most frequently involved. (11-23) The distribution of cardiac involvement in sarcoidosis is demonstrated by Figure 1 (24-41) while the clinical presentation of cardiac sarcoidosis (CS) has been summarized in Table 1. (12,13) The incidence and severity of cardiac involvement differs between ethnic groups, with higher incidence in Scandinavians and more severe cardiac involvement in Japanese and African-Americans. $(3,23,43-44)$ It is estimated that approximately $1-5 \%$ of sarcoidosis patients die of the condition, most commonly due to cardiac or pulmonary involvement. (3) The number of publications on CS has markedly increased over the last decades, largely due to the development of diagnostic imaging as well as the introduction of the cardiac implantable devices (CID), such as the pacemaker (PM) implantable cardioverter defibrillator (ICD) and catheter ablation of arrhythmias. (Figure 2) Table 2 (Figures 3-8) summarizes the contributions of the different relevant diagnostic modalities for CS. (45-136) The aim of this review is to provide relevant clinical and pathophysiological background on CS, whilst introducing cardiovascular magnetic resonance imaging (CMR) and detailing its role in the management of CS.

\subsection{Histopathology of cardiovascular sarcoidosis}

Inhaled antigens result in granulomatous alveolitis and activation of regional hilar and mediastinal lymph nodes. $(3,8,137,138)$ Activated lymphocytes and macrophages from regional hilar, mediastinal lymph nodes, and spleen circulate, and enter the myocardial interstitium. Micro vascular infiltration of lymphocytes and macrophages occurs at capillary level, with degradation and lamellation of the basal membrane, deformation of endothelial cells, resulting increased permeability. (139) A recent Japanese CS study confirmed the predominance of M1 pro-inflammatory macrophages and myeloid dendritic cells, which capture antigens in peripheral tissues and stimulate naïve T cells and induce T1 or T2 dif- 
ferentiation in the lymphocytic layer outside myocardial granulomas. (77) In addition antigens may enter the circulation with initial hematogenous spread followed by interstitial lymphogenic myocardial spread with local invasion. $(3,44,140)$ Resident dendritic cells engulf the antigen, and present it to CD4 T1 helper cells via the Major Histocompatibility Complex (MHC) class II receptor, initiating a pro-inflammatory response, with release of interleukins (IL1 $\beta$, IL2, IL6, TNF $\alpha$, IL12, IL18, and IFN $\gamma$ ), and chemokines. $(\mathbf{2 0 , 1 3 8 , 1 4 1 )}$ At least 3 different resident cardiac macrophage/monocyte subsets exist in the human heart, two of which are innate, embryological in origin. The resident macrophage subsets originate from bone marrow and the embryonic yolk sac, have anti-inflammatory, proregenerative capabilities, and persist by in-situ self-renewal. (142) An abundance in macrophages exists in the distal atrio-ventricular node, where they play a role in immune surveillance, with removal of infectious particles and cellular debris, myocardial regeneration and repair, and have the ability to conduct electrical signal via the gap junction connexin 43 . (143) Activation of these nodal macrophages in CS may in part explain the development of atrio-ventricular conduction disease. Activated macrophages turn into epitheloid cells, which merge and form multi-nuclear giant cells. (138) The basal septum, right-sided interventricular septum, lateral left ventricular (LV) wall and anterolateral papillary muscle have the most extensive lymphatic capillary network and are most frequency affected by granulomatous infiltration. (Figure 9) Obstruction of lymph drainage or retrograde lymphogenic spread of activated lymphocytes and macrophages from the right paratracheal lymph node, which drains the basal septum, right-sided interventricular septum, lateral left ventricular (LV) wall and anterolateral papillary muscle, may explain the predominance of myocardial involvement of these segments. (Figure 10) Activated macrophages produce lysozyme, angiotensin converting enzyme (ACE), biopterin and osteopontin. Figure 11 illustrates the progress through different stages of sarcoidosis involving the myocardium. Initially infiltration of CD4 lymphocytes causes interstitial edema, followed by migration of dendritic cells, and macrophages, which result in the formation of ill-defined interstitial granulomas. (stages I and II- exudative stages, Figures 11A and B). (19,20) Vascular involvement mainly consists of granulomatous infiltration of the media and adventitia of the small intramural coronary arteries and venules, whilst proliferation occurs of the intimal cells. $(\mathbf{1 4 4 , 1 4 5 )}$ Resulting myocyte degradation with small amounts of central necrosis in granulomas may account for the elevated highly-sensitive troponin levels, which have been reported to respond to treatment with corticosteroids. $(\mathbf{8 , 1 8 , 5 9 , 6 3 )}$ The activated proinflammatory macrophages turn into secretary epitheloid cells (activated macrophages resembling "epithelial cells" seen in intense immunological reactions - elongated in shape, with pale eosinophilic, finely granular, cytoplasm, and a central elongated nucleus). Fusion of epitheloid cells result in multi-nucleated giant cells (MGCs). MGCs are generally of the Langhans type, $4-50 \mu \mathrm{m}$ in diameter, with a large mass of cytoplasm, and a peripheral arrangement of at least 20 nuclei. MGCs may produce lysozyme and ACE. $(\mathbf{1 3 7 , 1 4 6 )}$

Stage III consists of relatively well defined non-necrotizing granulomas with abundant central epitheloid cells, MGCs, few central but plenty peripheral CD4 lymphocytes surrounded by a peripheral ring of CD4 and CD8 lymphocytes, plasma and mast cells. (Figures 4 and 11C) The peripheral CD4 lymphocytes demonstrate a shift towards Th2 cytokine profile with production of IL4, IL5, IL10, IL13 and TGF $\beta$, which are anti- 
inflammatory and result in a fibro proliferative response with extracellular matrix deposition. $(\mathbf{7}, \mathbf{1 3 8 , 1 4 6 )}$ Non-specific intra-cytoplasmic inclusion bodies may be observed in some MGCs. Asteroid bodies are stellate shaped inclusion bodies, 5-25 $\mu \mathrm{m}$ in size, of filamentous micro tubular material. Schaumann or Conchoidal bodies are oval, concentrically laminated bodies, generally $25-200 \mu \mathrm{m}$ in size, and consist of calcified proteins. Elongated birefringent crystalline cholesterol esters measure 1-20 $\mu \mathrm{m}$ in size. And lastly HamazakiWesenberg bodies, which measure 3-15 $\mu \mathrm{m}$ in diameter, which are giant intra and extra cellular lysosomes. $(\mathbf{8 , 2 0 , 1 3 7 )}$ Granuloma formation is a strategy during with the immune system aims to sequestrate particles, microbes, or pathogens which evade effective phagocytosis and killing. (20) Table 3 summarizes the specific and differentiating characteristics of granulomatous myocarditis. (147-152)

The final stages (IV) of CS consist of peri-granulomatous fibro-hyaline replacement with deposition of collagen. (Figure $11 \mathrm{C}$ and D) $(\mathbf{7 , 1 9})$ Overt evidence of necrosis of the cardiomyocytes as seen in idiopathic giant cell myocarditis (IGCM) does not occur in CS. (7) Cardiomyocytes atrophy, loose striations, with electron microscopic evidence of mitochondrial disruption, swelling, and loss of mitochondrial enzymes. Hyaline sclerosis, mucoprotein and glycoprotein deposition usually starts at the periphery of the tubercle, and gradually replaces the epitheloid cells. $(18,147,153)$

\subsection{Principles of Cardiovascular Magnetic Resonance and the application in cardiac sarcoidosis}

Spinning atomic nuclei with odd numbers of protons, such as hydrogen $(\mathrm{H})$, have magnetic momentum, and can be used for CMR. In the presence of a strong external magnetic field of a clinical CMR scanner (B0, 0.5-3 Tesla, 5000-50 000 fold the strength of the earths magnetic field) the parallel and anti-parallel spinning $\mathrm{H}$ protons align along the bore $(\mathrm{Z}$ axis) of the scanner. Slightly fewer align in the higher energy anti-parallel state, resulting in a net magnetization vector aligned to the external magnet. Individual nuclei precess around the direction of the external field in a phase that is different for each individual nucleus. When applied intermittent electromagnetic field pulses (pulse sequences) carry a radiofrequency which is identical to that of the $\mathrm{H}$ nuclei (Larmor frequency, $63.75 \mathrm{MHz}$ at 1.5 Tesla), energy is transferred which causes them to jump to the antiparallel state, precess in phase, and flips them into the X-Y plane to a degree determined by the flip angle of the pulse sequence. When the radiofrequency current is switched off, the nuclei relax and realign to $\mathrm{B} 0$, generating a radio frequency $(\mathrm{RF})$ signal, which is detected with receiver coils (a surface coil placed over or around the tissue or organ of interest or the magnet bore). The longitudinal, spin-lattice (surroundings) relaxation time (T1) describes how quickly the net magnetization vector recovers to its ground state in the direction of the external static magnetization field $\mathrm{B} 0$. The $\mathrm{T} 2$ or the spin -spin relaxation time relates to the decay of transverse magnetization (signal vector in the transverse $\mathrm{Y}$ axis/plane) after the resonance frequency pulse, and results from natural interactions at atomic and molecular levels. T2* (star) reflects the inhomogeneity's in the main magnetic field and field distortions produced by the tissue which cause the transverse magnetization to decay faster than predicted by 
natural atomic and molecular mechanisms (T2).Three constantly changing orthogonal magnetic gradients allow for the spatial localization of emitted radio waves and the reconstruction of images of the human body. To create an image the magnetic resonance signal from the H-protons needs to contain information about where the protons are positioned. By adding a small magnetic gradient along the main magnetic field in caudal to cranial direction an imaging slice can be selected. Because the frequency of precession of the protons depends on the local field strength, a narrow band of frequencies will only excite a thin slice of spins through the body. With change in excitation frequency another parallel slice can be acquired later. By using a combination of gradients in all three directions, it is possible to acquire a slice in any direction through the body. Frequency and phase encoding are used to obtain information for the individual points within a slice, or pixels. For the phase encoding a short temporary change in the magnetic field is applied between the radiofrequency $(\mathrm{RF})$ excitation pulse and the signal read out. This change will influence the frequency of precessing, resulting in a shift in the phase of precessing of the spins, dependent on the duration of this switch. By repeating this process with different duration of the temporary gradients, signals with different phase encoding are acquired. Frequency encoding is used to differentiate pixels with the same phase encoding. A magnetic gradient during read out of the signal results in a specific shift of the resonance frequency, likewise the effect of the slide-encoding gradient, for pixels with the same shift. Combining phase and frequency information allows the creation of a grid in which each pixel has a defined combination of phase and frequency codes. With a Fast Fourier Transform in both the frequency and phase encoding the raw digitized data are transformed into an image. The image resolution is determined by the obtained field of view (FoV, generally $240-400 \times 240$ $400 \mathrm{~mm}$ ), the number of frequency encodings (128-512 on the $\mathrm{X}$-axis), the number of phase encoding steps (128-512 on the Y-axis), and the selected slice thickness $(6-10 \mathrm{~mm}$, with/without gaps). In practice the image resolution is not determined by the pixels (picture elements, $0.5-2 \times 0.5-2 \mathrm{~mm})$ or voxel sizes $(0.5-2 \times 0.5-2 \times 6-10 \mathrm{~mm})$, but signal-tonoise ratio (SNR). Decreases in voxel size are generally associated with decreases in SNR and increases in acquisition time. The operator will need to individualize the acquisition settings for each individual patient as to answer the clinical questions. Image contrast is determined by intrinsic tissue magnetic longitudinal (T1) and transverse (T2) relaxation times determined by the type and structure of the atoms. Protons in different tissues produce signal at different relaxation rates, resulting in unique imaging signatures of different tissues. The signal strength is determined by the strength of the external magnetic field $\mathrm{B} 0$. Most cardiovascular studies are performed with 1.5 Tesla scanners/magnets. Although 3T decrease the acquisition time, increase signal strength and SNR, these advantages may be off set by the higher susceptibility to artifacts. When one uses more than one RF pulse, a so-called pulse sequence, one can use pulses with different flip angles $\left(90^{\circ}\right.$ or $\left.180^{\circ}\right)$. The flip angles of the pulses and the time between the pulses will determine the tissue signal. The time between the pulse sequences are called time to repeat (TR). TE is time to echo, which can be chosen by the operator, influencing the resulting signal and thus the image. The shorter the TE time, the stronger the signal, the better the signal-to-noise ( $\mathrm{SN}$ ) ratio, but the weaker the contrast. The longer the TE the more T2 weighted the study. By manipulating the number of pulses, their flip angles, the TR, and TE the CMR operator can 
generate T1 weighted, T2 weighted or proton-weighted images. By altering the timing and strength of the alternating gradients, as well as the administration of external contrast agents, which alter the longitudinal relaxation times or spoil magnetization, differences in T1 and T2 values in tissues can be exploited to enhance and differentiate specific tissues of interest. Rapid imaging sequences allow for real time imaging, but most images requiring high spatial resolution and tissue contrast require electrocardiographical (ECG) timing to allow for the sampling of imaging data over several cardiac cycles. The large majority of investigations are performed with 2D imaging, with the acquisition of one slice of data in one breath-hold (generally lasting 11-20 seconds). Navigator triggered free breathing imaging may be considered in patients who are unable to hold their breath. CMR contrast agents predominantly alter the $\mathrm{T} 1$ relaxation time of the $\mathrm{H}$ nuclei resulting in stronger i.e. brighter signal. ECG triggered bright-blood sequences (b-SSFP) allow for functional assessment of myocardial segmental and global systolic left and right myocardial functions. $(153,154,155)$ Tables 4 (Figures 12-16) (38,57,99 132,134,158-171,175-179) and 5 (Figures 12-20) $(27,28,31,32,33,35,36,38,39,161,163,164,167,175,180,181,183-185)$ summarize the CMR sequences used in the evaluation of patients with suspected CS. The Society of Cardiovascular Magnetic Resonance (SCMR) has published protocols, which aim to optimize and standardize the evaluation of non-ischemic cardiomyopathies (NICM) and include recommendations for the assessment of patients with suspected CS. $(\mathbf{1 5 7 , 1 5 8 )}$

\subsection{Contrast agents and their application in the evaluation of cardiac sarcoidosis}

Contrasted CMR is mainly used to detect active granulomatous inflammation and chronic focal or diffuse interstitial scar. (Figure 14) $\mathbf{( 1 6 8 , 1 7 1 , 1 8 3 - 1 8 8 )}$ Although first-pass perfusion may be used to evaluate myocardial perfusion deficits due to coronary vasculitis or microcirculatory obstruction due to infiltrating granulomas, this is not generally used in clinical practice as it does not improve diagnostic or prognostic accuracy in CS. (187) The normal intra-cellular compartment contains approximately $75-80 \%$ of myocardial volume, with the remaining $25-30 \%$ representing the interstitial volume. Increased capillary permeability, interstitial expansion due to cellular, granulomatous inflammation, lymphatic obstruction and fibrosis due to collagen deposition result in an increased interstitial volume of distribution with decreased washout of the gadolinium-based contrast agents (GBCA). (Figure 21) $(130,131,156-158,167,170,185,188,189)$ Table 6 summarizes the GBCA used in CMR. (185) GBCA consist of gadolinium, a biologically inert water soluble tracer obtained from rare earth, linked to different ligands such as diethylene- triaminepentaacetic acid (DTPA), which render it the capabilities to distribute freely in the intravascular and extra-cellular interstitial space. Gadolinium is the critical paramagnetic element responsible for the enhancement properties of GBCA, but the chemical structure of the ligand determines the degree of enhancement, half-life, distribution, pharmacokinetics and toxicity. GBCA do not cross the cell membrane unless damaged by ischemia, toxins, or as in CS, inflammation. Histopathological studies have revealed evidence of limited central cardiomyocyte necrosis in which case GBCA will accumulate intra-cellularly. (165) With the 
clinically used intravenous dosages of $0.1-0.2 \mathrm{mmol} / \mathrm{kg}$ of GBCA the longitudinal (T1) relaxation time is shortened, with little effects on the T2 relaxation time. (186) Most investigators evaluating CS have used gadolinium-diethylenetriaminepentaacetic acid (Gd DTPA), gadopentetate dimeglumine, gadodiamide or gadoversetamide at $1.5 \mathrm{~T}$ field strength with dosages differing from $0.1-0.2 \mathrm{mmol} / \mathrm{kg}$. Few have used gadobenate dimeglumine (BOPTA), with higher reported T1 signal. The myocardial T1 with BOPTA is 15 msec shorter without significant difference of the T1 relaxation time in the blood pool. This results in slightly greater extra-cellular volume (ECV) measurements with Gd-DTPA in the order of 0.01 , greater T2 relaxivity in blood plasma and interstitial fluid, with higher contrast-to-noise (CNR) and signal-to-noise ratio (SNR) of LGE compared to remote normal myocardium. Lower dosages of BOPTA are needed compared to Gd-DTPA to deliver same diagnostic quality. Gd-BOPTA also prolongs the enhancement of blood, which can result in lower sensitivity for small subendocardial scars. The higher intra- and inter-observer variability with Gd-BOPTA, may however leave Gd-DTPA the preferred agent. (191) The use of GBCA carries a very low rate of adverse events (0.01-2.4\%), lower compared to low osmolar non-ionic iodinated contrast agents for computed tomography.

\section{(191-193)}

The most relevant safety issue is its restricted use to patients with preserved renal function. Nephrogenic systemic fibrosis (NSF) has been reported in a small subgroup of patients with an estimated glomerular filtration rate $<30 \mathrm{ml} / \mathrm{min} / 1.73 \mathrm{~m}^{2}$ The FDA has published guidelines for the use of GBCA in this population. (194)

\subsection{Determining focal fibrosis (Tables 4, 5)}

Until the introduction of inversion-recovery (fast) gradient echo (IR-GRE) sequences the delayed enhanced spin echo (SE) sequence was used to determine the presence, localization, distribution and amount of focal fibrosis i.e. LGE in ischemic and non-ischemic cardiomyopathies. (161,163,164,195-197) (Figures 12,14,15) IR-GRE sequences consist of a conventional SE sequence preceded by a $180^{\circ}$ inverting pulse. The time between the inverting pulse and the $90^{\circ} \mathrm{SE}$ pulse is called the inversion time (TI). Ten to fifteen minutes after the administration of the GBCA the inversion pulse is administered. The function of the inverting pulse is to flip the initial longitudinal magnetization (M0) of all tissues in the imaged slice or volume to the point opposite to the direction of the main magnetic field (B0). During the TI interval these inverted tissues undergo T1 relaxation as they variably seek to re-establish magnetization along the Z-axis. At the $90^{\circ} \mathrm{SE}$ pulse the longitudinal magnetizations of different tissues are now separated based on their different intrinsic T1 relaxation times. Varying the TI parameter controls the degree of separation and thus image contrast. The TI is generally optimized on individual basis, ranging from 200-360 msec, and chosen as to suppress ("null") signal from normal, un-enhanced, myocardium so that contrast with the bright, contrast-enhanced myocardium is optimal. The TI is timedependent - as time lapses from administration the TI may need adjustment. The PhaseSensitive Inversion Recovery (PSIR) acquisition sequence obviates the need for adjustment, it has been designed to null the myocardium over a range of inversion times. Generally two 
separate phase encoding directions and two orthogonal DECMR views are generated confirm true LGE and to exclude artifact. (Figure 22) Diffuse interstitial inflammation (early stage of CS) or diffuse fibrosis will not be visualized by IR-GRE sequences, as the areas with little interstitial gadolinium will be nulled compared to the areas containing most gadolinium.

LGE by CMR has become the gold standard for assessing acute myocardial infarct scar (AMI), chronic myocardial infarct scar (CMI), and focal scar in NICM such as CS. (Figures 20, 23)

Although IR-GRE sequences have become the acquisition sequence of choice, there is currently no uniform approach for the CMR quantification of focal myocardial fibrosis in NICM.

Investigators have used different amounts of a variety of contrast agents (single vs. double dose 0.1-0.2), different delays between administration of contrast agents and the acquisition of images, different inversion times - fixed or individual optimization, different slice thicknesses (thicker slices, higher SNR), magnetic field strengths (higher strength, higher SI, higher SNR, CNR), and different methods of quantifying the amount of LGE visual interpretation with manual planimetry, semi-automated methods using signal intensity (SI) at a fixed number of standard deviations (SD, 2 - 6 SD) above remote unenhanced myocardium, subjective segmental scoring by independent experienced readers or the fullwidth at half-maximum method (FWHM) whereby the amount of LGE is delineated at $50 \%$ of the maximum SI in enhanced myocardium. $\mathbf{( 5 7 , 1 3 4 , 1 9 8 , 1 9 9 - 2 0 1 )}$ The reproducibility of LGE in NICM seems worse compared to ischemic scar, and LGE volume may vary substantially depending on the condition studied and quantification technique used. (202) The $>2$ SD technique generated LGE volumes up to two times higher compared to the manual planimetry, FWHM, and $>6$ SD methods. Regardless of the disease under study the FWHM method gives LGE volume similar to the manual quantification and seems most reproducible, reducing required sample size by half. (200)

Currently the recommended reporting of myocardial scar with $>2$ SD above the signal of mean remote unenhanced myocardium in the same slice. (203) LGE of the relatively thin walled right ventricle (RV) was visually assessed by dividing the RV in 12 segments.

(204) (Figures 7,8,9, 12)

\subsection{The measurement of extracellular volume and diffuse fibrosis with T1 mapping}

The normal extra cellular myocardial volume (ECV) measures approximately $23 \%$ and extra cellular matrix expansion by edema, interstitial fibrosis, deposition of iron, lipid, collagen, amyloid, or cellular infiltration as determined by the use of native and post-contrast T1 maps is diagnostic of myocardial dysfunction and an independent biomarker of poor outcome. (Figure 21) Native T1 mapping and the assessment of the ECV by the use of native and post-contrast T1 maps are currently considered as the most promising methods and may also detect myocardial ischemia, and myocyte necrosis due to ischemia in acute infarction. (189,205-213,216,228) T1 mapping refers to the pixel wise quantification of the 
myocardial longitudinal relaxation time. Global T1 measurements can be determined either by analyzing the intensity of a group of pixels in a parametric T1 map, but also by deriving an exponential T1 recovery curve based on regions of interest (ROIs) from a number of SSFP images with varied inversion times i.e. (Shortened) MOdified Look-Locker inversionrecovery sequences (MOLLI, ShMOLLI) and saturation recovery sequences SAturation recovery Single-sHot Acquisition (SASHA). Many widely used myocardial T1 mapping sequences use breath-hold acquisitions that limit precision - free breathing high-contrast SASHA (SASHA-HC) may provide accurate T1 maps with higher precision than MOLLI in acquisitions less than 30 seconds. Native (contrast-free) myocardial T1 mapping with MOLLI, ShMOLLI evaluates the whole myocardium, including capillaries, myocytes, and blood, and is able to detect and quantify myocardial ischemia, and extra-cellular expansion due to edema, collagen, amyloid, lipid or iron deposition on a pixel by pixel basis. (Figures 15,16) Since it obviates the need for contrast agents it can be applied in patients with renal impairment, is heart rate independent resulting in less image artifacts, and is less reliant on reference values. Standardization is however an issue, and locally derived normal values need to be determined in each patient population. Variability is introduced when determining ECV by different contrast agents and field strengths. Usually acquisition is obtained in end-diastole but systolic acquisitions, with lower T1 values, are more optimal in AF. Relatively fast breath-hold T1 mapping like MOLLI have made assessment of ECV a reality. Stable measurements are generated 5-10 minutes after a bolus of contrast agent.

The ECV fraction is derived from the ratio of changes in pre and post contrast signal in the extracellular myocardium and extracellular LV blood pool water (plasma). $(205,207,209,210,212)$ The conventional approach requires a blood test to determine hematocrit $(\mathrm{Ht})$ and post-processing integrates the $\mathrm{Ht}$ in calculations of the ECV (\%). The inverse linear relationship between the Ht and the native blood pool T1, allows for noninvasive estimation of the Ht. (214) Recently several investigators demonstrated the feasibility of estimating the ECV based on this synthetic, non-invasive, pre-contrast blood pool T1 based ECV, which allowed an immediate inline ECV map to be generated. (215-217) T1 and ECV measurements will likely very helpful in the diagnosis, management and prognostication of CS, due to its ability to evaluate focal or diffuse intra- and extracellular interstitial disease processes. $(\mathbf{1 1 2 , 1 7 1 , 1 7 3 , 2 1 8 )}$.

This technique is based on the change in T1 values after injection of gadolinium DTPA, an extracellular contrast agent. ECV is estimated from repeated native T1 acquisitions at baseline and at 10 minutes after contrast administration. The change in 1/T1 in tissue and blood pool is used to determine the contrast agent concentrations, the ratio of which is an estimation of ECV, followed by a correction of red cell concentration in the blood pool (hematocrit (Ht)). The Ht can be estimated from the native values of blood T1 precluding the need for laboratory tests.

Motion between the images will adversely impact T1 measurements, and should be corrected.

Relatively fast breath-hold T1 mapping like MOLLI have made assessment of ECV a reality stable measurement 5-10 minutes after bolus of contrast agent. Measuring the ECV is superior to native or post-contrast T1mapping in determining collagen volume fraction 
and risk assessment in large cohorts, and has been well-validated and highly reproducible across different vendors.

Confounders for post-contrast T1 mapping are renal clearance, contrast relaxivity, body weight, anemia, and the time between acquisition and administration of the contrast-bolus, $(219,220)$

\subsection{CMR assessment of myocardial inflammation in sarcoidosis}

Several case-reports and studies have reported on the value of SE T2 weighted, fat and blood suppressed T2 weighted (spectral presaturation with inversion recovery, SPIR; short tau inversion recovery, STIR), early relative and late post-contrast T1weighted imaging in patients with CS. (30,122,131,132,159-161,175,197,221-224) (Figure 13).

Due to the patchy distribution of the disease and lack of histological correlation the sensitivity and specificity of these sequences for the diagnosis of CS are unknown. T2 weighted imaging detects edema but is prone to low-signal-to-noise motion artifacts, imperfect blood suppression in areas of slow blood flow, and subjective visual interpretation. $\mathbf{( 1 7 4 , 2 2 4 )}$ Hypo intense lesions on T2 weighted imaging signify fibrosis, while LGE demonstrates both active inflammation as well as focal fibrosis. $\mathbf{( 3 8 , 1 6 5 , 1 8 9 )}$ (Figure 14) The concept of relative early enhancement (ERE) consists of comparing the signal intensities (SI) in regions of the left ventricle to skeletal muscle, such as the major pectoral or paraspinal muscles, 4 minutes after administering GBCA using SE black blood imaging. An increased regional global signal intensity (SI) in the myocardium signifies expanded interstitium reflecting inflammation by edema (relative SI $\geq 2$ ) and hyperemia (relative SI $\geq 4$ ) and, although no direct comparisons have been made, in all likelihood may be similar findings with T1 and T2 mapping. (38,165,175,189,224).

Regional myocardial T2 or transverse relaxation time mapping overcomes the challenges of image quality, reproducibility, and subjective assessment. Images are acquired with different echo times and are used to estimate the T2 values. Although the spatial resolution with T2-STIR is finer, allowing for more detailed subjective focal disease inspection, T2 mapping is heart rate independent, resulting in fewer artifacts, and less reliant on reference values. (224) A parametric image or map is generated with each pixel reflecting the calculated T2 relaxation time. Maps can be analyzed visually on a grey or color scale and regions of interest can be drawn for quantitative analysis. (Figure 16) At least three single-shot images are generated with increasing T2 preparation times to construct a relaxation curve, with long repetition times to prevent $\mathrm{T} 1$ longitudinal relaxation from distorting the images. Crouser used T2 mapping in a retrospective analysis comparing 50 suspected CS patients to healthy controls, whilst using a cut off value of $59 \mathrm{~ms}$ based on previous studies in viral myocarditis, and determined that mapping had a sensitivity of $54 \%$ and specificity of $100 \%$ for the diagnosis of CS. (171) In 41\% of patients global T2 was prolonged in the absence of LGE, indicative its superior sensitivity.

The combination of these different sequences potentially helps distinguish inflammation from chronic sequellae. $(38,164,165,171,174,189,213,216,222,224,225,228)$ The assessment of active granulomatous myocardial inflammation is important for diagnostic, 
treatment and prognostic perspectives. Active granulomatous myocardial inflammation appears more arrhythmogenic and may potentially respond to immune-suppressive treatment. $(11,56,79,115,116,174,221,230,231)$.

Positron Emission Tomography (PET), Single Photon Emission Computed Tomography (SPECT) and Single Photon Emission Computed Tomography Dual Isotope Scintigraphy have been demonstrated useful in CS, with FDG, Octreotide and ${ }^{68} \mathrm{Ga}$-DOTA-TOC the most sensitive tracers. (111,112,122,126,174,231,233) (Figures 6A,7B,8) PET CT is currently considered the most sensitive modality for active CS. $(\mathbf{1 1 5}, \mathbf{1 2 1}, \mathbf{1 2 2 , 1 2 6 , 1 3 1 , 1 7 4 , 2 3 2 , 2 3 3 )}$ Isotope scintigraphy is particularly useful when CMR is contra-indicated, but exposes the patients to ionizing radiation and is limited by image resolution. $\mathbf{( 2 2 4 , 2 2 6 )}$ The high sensitivity of FDG-PET for myocardial inflammation, and the superior image resolution, functional and structural information of modern CMR (Table 2) combined, make hybrid FDG PET/CMR the diagnostic and management tool of choice. (124-130,234,235) The relatively thin RV wall currently precludes breath-hold parametric mapping, but respiratory-gated techniques may provide a future solution. (Figure $7,8,12)$

\subsection{The potential of Ultra Small Super paramagnetic and Nano particles in the assessment of mononuclear myocardial inflammation}

Ultra small super paramagnetic particles of iron oxide (USPIOs) consist of an iron oxide core surrounded by a carbohydrate or polymer coating, small ( $<20$ to $250 \mathrm{~nm}$ ) enough to extravasate through diseased microvasculature, where they may be engulfed by tissue resident macrophages.

Accumulation of USPIOs reduces the T2* decay time and creates signal deficits that can be visualized and quantified by $\mathrm{T} 2 * \mathrm{CMR}$. Thus the cellular inflammatory process can be imaged at tissue level. This technique can provide a non-invasive method for the diagnosis and monitoring of the tissue macrophage activity in the myocardium, and potentially allow for the evaluation of novel therapeutic interventions. (236) The current limited preclinical and clinical data confirm the potential of both magnetic and perfluorocarbon nano particles for the diagnosis of active myocarditis with histological correlation of imaging signal. $(\mathbf{2 3 7}, \mathbf{2 3 8})$ In addition to iron oxide particles, intravenous perfluorocarbon nano particles ${ }^{19} \mathrm{~F}$ MRI may become useful in diagnosing and monitoring inflammatory cells macrophages, dendritic cells, granulocytes - in the myocardium. In contrast to the regular ${ }^{1} \mathrm{H}$ NMR imaging fluorine particles are virtually absent in the human body, which means the absence of background noise -resulting in high specificity. Immune cell infiltration was successfully imaged in the pre-clinical beating mouse heart model of autoimmune myocarditis with a high field research magnet of $9.4 \mathrm{~T}$ with simultaneous acquisition of ${ }^{19} \mathrm{~F}$ and the matching ${ }^{1} \mathrm{H}$ images. This depicts hot spots of ${ }^{19} \mathrm{~F}$ phagocytized by circulating macrophages, and creates the possibility of early detection and delineation of inflammation. Since these technologies are highly specific and have the ability to quantify they hold great promise for the diagnosis and management of sarcoidosis. $(\mathbf{1 7 4 , 2 3 9 , 2 4 0 )}$ 
A substantial percentage of CS receives treatment with (biventricular) pacemakers (PMs) or implantable cardioverter defibrillators (ICDs). $(\mathbf{9 5 , 9 6 , 2 4 1 )}$ Off-label CMR scans in non-conditional pacemaker dependent patients and those with implantable cardioverter defibrillators for relevant diagnostic or management reasons can be safely performed with use of a strict protocol. (239,240,244,245) Since 2008 MRI compatible ('conditional') leads and pacemakers have become available, further reducing the extremely low risk of device interference $(0,3 \%)$ by the radiofrequency energy and magnetic fields. $(244,245)$

\subsection{Cardiovascular Magnetic Resonance in cardiac sarcoidosis: the first publications}

The first case-reports describing the application of uncontrasted T1 and T2-weigthed spin echo imaging were by Riedy et al in 1988 (180) (Figure 17) and Dupuis et al in 1994. (245) Chandra in 1996 were the first to report segmental LV dysfunction on bright blood ('cine') imaging and mediastinal lymphadenopathy in a patient with dilated cardiomyopathy secondary to CS. (246) The authors reported superior image quality and suggested CMR should be considered for diagnostic and therapeutic monitoring purposes in the each patient with suspected CS. In 2001 Shimada et al were the first to report the diagnostic findings and response to steroid therapy with gadolinium-enhanced CMR in 8 of 16 sarcoidosis patients. (162) Vignaux et al were the first to report the long-term response to steroid treatment with more comprehensive CMR imaging including cine imaging, and (un)contrasted T1 and T2 -weighted studies. $(\mathbf{1 5 9 , 1 6 0 )}$

\subsection{The CMR diagnosis and differential diagnosis of cardiac sarcoidosis}

The definite diagnosis of CS is based on histological confirmation by EMB, but due to the patchy, predominantly epicardial and mid-myocardial, LV distribution of granulomatous sarcoid infiltration, RVseptal EMBs suffer from sampling error and may be "false negative". (75-77, 80,81,247,248) (Figure 24) In the absence of diagnostic EMBs the current guidelines allow for the "clinical" ( "at least probable", or "possible") diagnosis of CS to be made when ECG (conduction disease, supra/ventricular arrhythmias) and/or (noninvasive) diagnostic imaging (CU, PET/CT, Tc-MIBI, Thallium, Gallium, DECMR, delayed enhanced MDCT, invasive ventriculography) reveal abnormalities associated with CS (segmental myocardial motion abnormalities, hypertrophy, atrophy, focal scar, uptake, reversed perfusion defects) in the presence of histologically confirmed extra-cardiac sarcoidosis. Alternative conditions, such as hypertrophic cardiomyopathy (HCM), arrhythmogenic right ventricular cardiomyopathy (ARVC), amyloidosis, Chagas disease, viral myocarditis, dilated cardiomyopathy (DCM), or coronary artery disease (CAD), need to be excluded. (11,46,152,163,164,168,178,218,249-256) (Table 7) (Figures 23,24,25,26) The diagnosis of CS is certain when the EMB demonstrates the histopathological hallmark of this condition, non-caseating granulomas in the absence of etiologic agents, microbes or fungi. 
Although DECMR or electrophysiological endomyocardial mapping guided EMBs have decreased sampling error, the mid-myocardial or epicardial LV distribution of granulomas limits the diagnostic accuracy of trans venous EMBs. (74) (Figure 20) In the absence of diagnostic endomyocardial histology DECMR and PET have been demonstrated to be the most accurate diagnostic imaging test in patients with diagnostic extra-cardiac histopathology. (11,12,257,258) Table 2 describes the advantages and limitations of the different diagnostic modalities used until now. DECMR has rapidly developed as the most useful. Lately the number of (peer-reviewed) publications has steadily increased from 10-15/year to 100150/year. (Figure 2)

\subsection{Isolated cardiac sarcoidosis}

Cardiac sarcoidosis without clinically apparent extra-cardiac disease so-called "isolated cardiac sarcoidosis" (ICS) has recently gained more attention and acceptance as a clinical entity. $(259,260,261,262)$ Although the true prevalence of ICS is not known due to its inherent diagnostic challenge, estimates range from to $5.5-25 \%$ of CS. $(\mathbf{9 5 , 2 6 2 )}$ ICS may be more prevalent amount Scandinavian (Finish) and Asian (Japanese) sarcoidosis patients. $(259,262,263)$ A thorough clinical work-up of patients with suspected sarcoidosis including delayed enhanced CMR and whole-body F-18-fluorodeoxyglucose Positron Emission Tomography (FDG-PET) guided sampling of (mediastinal/hilar) lymph nodes and right or left ventricular endomyocardial biopsies (EMB) markedly increases diagnostic accuracy for histologically confirmed CS. (259,262,264,265) The current HRS (2014) and JSSOGD (2006) guidelines do not provide for the diagnosis of ICS i.e. CS in the absence of histological evidence of extra-cardiac or cardiac sarcoidosis. The definite diagnosis of CS is based on histological confirmation by EMB, but due to the patchy, predominantly epicardial and mid-myocardial, LV distribution of granulomatous sarcoid infiltration, RV septal EMBs suffer from sampling error and may be "false negative". (11,266) It is urgently necessary to formulate evidence based diagnostic criteria for "ICS". Several recent publications included diagnostic guidelines for ICS based on imaging tests with major emphasis on contrasted CMR, whole body PET/CT, and hybrid PET/CMR. (95,261,263,267-271) When assessed with whole body 18FDG PET isolated CS was found to be rare, with only 1 of 31 CS patients without extra-cardiac uptake. PET and DECMR are the crucial diagnostic imaging tests used to diagnose CS in the absence of extra-cardiac or cardiac tissue diagnosis. $(95,262,267-271)$

\subsection{The significance of evaluating for mediastinal lymphadenopathy in cardiomyopathy}

Riedy et al (1988) were the first to report on CMR evidence of CS and mediastinal lymphadenopathy in a patient with CS. In patients diagnosed with idiopathic dilated cardiomyopathy and negative endomyocardial biopsies, computed tomography (CT) or PET/CT should be employed to assess for mediastinal lymphadenopathy. Due to sampling error 
endomyocardial biopsies (EMB) may be negative in 50-70\% of patients with CS. (75) Histological assessment of sampled mediastinal nodes may reveal underlying sarcoidosis as the potentially reversible etiology of the cardiac condition. $(264,265,274-276)$ A thorough clinical work-up of patients with suspected sarcoidosis including delayed enhanced CMR and whole-body 18FDG-PET guided sampling of (mediastinal/hilar) lymph nodes and RV or LV EMBs markedly increases diagnostic accuracy for histologically confirmed CS. $(259,262,264,265)$

\subsection{Role of cardiovascular magnetic resonance in the guidelines and position papers}

Sofar CMR has been included in the Diagnostic and Management Guidelines of 5 professional Societies. (11,46,251-254,266,277-279) (Table 8) Since the first Diagnostic Guidelines by the the Japanese Ministry of Health and Welfare in 1993 (JMHW) the prominence of CMR has increased from being a minor to becoming a major contributor to the diagnosis of CS with prognostic relevance. $(11,46,254,277,278)$

\subsection{Atrial sarcoidosis}

Supraventricular arrhythmias occur in up to $32 \%$ of patients diagnosed with CS, with atrial fibrillation $(18 \%)$ being most common and multifactorial in etiology. $(280,281)$ Despite the limited image resolution due to the atrial wall thickness only measuring $4 \mathrm{~mm}$, atrial infiltration and scarring can be demonstrated by T2 weighted and LGE CMR. Both have been reported to be the substrate of atrial fibrillation, flutter and sinus node dysfunction. $(\mathbf{2 4}, \mathbf{2 8 2}, \mathbf{2 8 3})$ Though not yet studied in CS, atrial CMR strain analysis may potentially provide additional valuable information in patients with (suspected) CS. Impaired atrial booster function may carry valuable diagnostic and prognostic information, representing evidence of (sub)clinical (atrial) CS, myocardial fibrosis and non-compliance, as well as longterm risk of SVT. (281-284)

\subsection{Right ventricular sarcoidosis}

Until recently limited attention has been given to RV involvement in CS, its prevalence, relevance and prognostic value. (285) CMR is the preferred imaging tool to diagnose and delineate RV disease. (286-288) RV volumes, mass, and function can be quantified without geometric assumptions, with excellent intra- and inter-observer agreement and good interstudy reproducibility. $(\mathbf{1 3 3 , 1 8 9 , 2 8 9 , 2 9 1 )}$ Although there are numerous reports on DECMR delineating LV sarcoidosis, relatively few studies have reported on RV involvement. (204,285,291-293) RV LGE is present in approximately $15 \%$ of patients diagnosed with pulmonary sarcoidosis, and in $38-49 \%$ of patients with left ventricular enhancement. 
$(29,30,285)$ (Figures 7,12) The presence of RV LGE correlates with pulmonary arterial hypertension (PAH), RV systolic dysfunction, hypertrophy and dilation. $(\mathbf{2 9 , 3 0 )}$ RV sarcoid means more extensive and active CS, and also more active extra-cardiac i.e. mediastinal and hilar sarcoidosis $\mathbf{( 2 9 , 3 0 , 2 9 2 )}$ (Figure 9) Biventricular LGE at presentation may be the strongest, independent predictor of adverse outcome during long-term follow up of sarcoidosis patients. $(\mathbf{3 0}, \mathbf{1 1 5}, \mathbf{2 0 4 , 2 9 2 , 2 9 6 )}$ Predominant or isolated RV involvement is rare, with the large majority of patients with RV LGE also suffering from LV disease. (28-30) More extensive LV LGE is associated with RV involvement. (134,189,204,295,298) RV LGE, and inflammation as demonstrated with FDG PET, have been associated with ventricular tachy-arrhythmias. (134,204,230,241,298) (Figures 7,8) The reported prognostic relevance of RV disease may at least partly reflect the extent of LV arrhythmogenic substrate. Several post-transplant and post-mortem studies in sarcoidosis patients have reported $\mathrm{RV}$ involvement to range from $6 \%$, in patients dying from alternate causes, to as high as $65 \%$ in those dying sudden cardiac deaths. $(\mathbf{1 6 , 1 8 , 1 4 5 , 2 9 9 , 3 0 0 )}$ Generally, patients with congestive failure were found to have extensive biventricular sarcoid, and in those who had died suddenly, active granulomatous infiltration and patchy scar were present. RV outflow tract involvement was rare. Eleven recent studies employing DECMR in the assessment of cardiac sarcoidosis, reported on RV disease in sarcoidosis. $(29,133,134,189,204,230,241,285,289,298,301) R V$ enhancement was reported in 6 of these studies, and ranged from $2 \%$ in unselected to $48 \%$ in high-risk populations. $(\mathbf{1 3 2}, \mathbf{1 8 9}, \mathbf{2 0 4 , 2 8 5 , 2 8 9 )}$ The presence of RV LGE correlates with hypertrophy, dilation and systolic dysfunction. (29) RV LGE in sarcoidosis may be caused by direct granulomatous infiltration, but also be related to PAH. (302) RV hypertrophy, dilation, dysfunction and LGE may be secondary to $\mathrm{PH}$, and are associated with worse prognosis. (302,303) (Figure 20) $\mathrm{PH}$ is found in $6-28 \%$ of the general sarcoidosis outpatient setting, and may be secondary to pulmonary fibrosis, angiitis and/or congestive heart failure. (303) (Figure 27) RV dilation and systolic impairment in sarcoidosis have been demonstrated to predict adverse outcome in sarcoidosis. $(\mathbf{1 0 5}, \mathbf{1 3 4}, \mathbf{2 0 4}, \mathbf{2 3 1}, \mathbf{2 4 1 , 2 9 8 , 3 0 1 , 3 0 4 )}$ In patients with predominant RV disease, CS needs to be differentiated from arrhythmogenic right ventricular cardiomyopathy (ARVC). (Figure 25) Distinguishing features favoring sarcoidosis consist of an older age of onset, a non-familiar pattern, wider QRS complexes, septal involvement with atrio-ventricular conduction disease, multiple arrhythmogenic foci, particularly RV apical tachycardia, concomitant LV disease, and the presence of mediastinal lymphadenopathy. (305) LGE of the septum and insertion points may result from delayed wash-out of gadolinium due to altered myocardial fiber strain, fiber disarray, ischemia, and fibrosis, secondary to RV pressure or volume overload, and resulting septal shift. $\mathbf{( 3 0 0 , 3 0 6 , 3 0 7 )}$ (Figure 27) Ventricular insertion point enhancement has been reported in HCM, atrial septal defects, severe PAH, tetralogy of Fallot, transposition of the great arteries and even a proportion of healthy veteran endurance athletes. (306-311) (Figure 23) The amount of insertion point enhancement correlates with mean pulmonary arterial pressures, RV mass, volume and ejection fraction. (300) PH in sarcoidosis is associated with adverse outcome, particularly when accompanied by RV dysfunction, and/or lung fibrosis. $(302,303)$ 


\subsection{First-pass myocardial perfusion and perfusion reserve in sarcoidosis}

Bogabathina was the first to report non-specific first-pass perfusion defects and widespread patchy LGE with DECMR in a patient with severe active CS. (169) Only a single study has systematically evaluated myocardial stress perfusion CMR in 43 asymptomatic sarcoidosis patients, 34 of which had evidence of diffuse interstitial fibrosis and an impaired CMR myocardial perfusion reserve index (MPRI). (312) Follow up of 18/34 patients a year later revealed an increased T2 ratio and LGE in 6/9 patients with initial evidence of myocardial inflammation. Treatment with corticosteroids resulted in a normalized CMR scan at 6 months follow up, without any cardiovascular symptoms or events during this period.

Kruse et al demonstrated regional impairment of coronary circulatory function in segments with sarcoid-mediated myocardial inflammation with ${ }^{18} \mathrm{FDG} /{ }^{13} \mathrm{NH} 3 \mathrm{PET} / \mathrm{CT}$. Effective immune-suppression mediated decrease in inflammation was associated with preserved myocardial flow reserve (MFR) at follow-up whereas MFR significantly worsened in regions without changes or even increases in inflammation. (313) Previous studies with dipyridamole thallium and technetium perfusion scintigraphy had demonstrated similar reversible perfusion defects at rest redistribution and after dipyridamole. $(\mathbf{1 1 0}, \mathbf{1 1 4}, \mathbf{2 5 1}, \mathbf{3 1 4})$ These findings suggest a subclinical functional myocardial micro vascular perfusion disorder, which may be amenable to immune-suppressive treatment. The clinical and prognostic relevance of these findings is currently unclear.

\subsection{Pericardial sarcoidosis}

Sarcoidosis rarely affects the pericardium, with only a few case reports describing granulomatous pericarditis with effusion, tamponade and constrictive pericarditis as a chronic sequellae. (39-42) DECMR allows for the assessment of pericardial thickness, inflammation, fibrosis, fluid and septal motion. (Tables 4 and 5)

\subsection{Macro granulomas}

Granulomatous proliferation may result in large inflammatory masses, most frequently infiltrating the interventricular septum. DECMR delineates the inflammatory nature, precise localization, size and response to immune-suppressive treatment of the contrastenhancing mass. $(33,180,183,184)$ (Tables 4,5) (Figure 17) 


\subsection{Can the distribution of LGE differentiate between different cardiomyopathies?}

\section{A pattern-based approach to assessment with DECMR in cardiomyopathies}

The distribution pattern of LGE in ischemic heart disease generally includes the subendocardium and may involve the mid- and epicardial layers, as ischemia and subsequent myocardial cell necrosis advances after occlusion of the perfusing epicardial coronary. $(\mathbf{1 6 8 , 3 1 5})$ The focal scar pattern in NICM is not restricted to the distribution of coronary arteries and does not progress from endocardial to epicardial. (168,178,315-318) Dilated cardiomyopathy may be differentiated from specific infiltrative or inflammatory cardiomyopathies by the predominantly linear and patchy mid-myocardial LGE. (168,178,315-318) The pattern of LGE in CS is patchy, multi-segmental, not related to coronary artery territories, predominantly involving the mid myocardial and subepicardial layers. Near transmural scar is present in a minority of patients. $(\mathbf{9 9 , 1 3 2 , 1 6 8 , 1 8 3 , 1 9 7 , 3 1 6 - 3 2 8 , 3 2 0 - 3 2 7 )}$ The pattern of LGE in CS needs to be distinguished from viral myocarditis, Anderson-Fabry and Chagas disease, which also predominantly affect the mid-myocardium and epicardium. (168,316-322) The clinical settings, symptomatology, (family) history, and geographical region will help determine the most likely etiology and differentiate between these conditions. In additional to LV enhancement RV dilation and LGE is present in up to $30 \%$ of CS patients, which may help differentiate CS patients from viral myocarditis, AndersonFabry and Chagas disease. (29,30,168,316-322) The junction of RV and LV and the rightsided interventricular septum are predominantly affected. $(\mathbf{2 9 , 3 0 )}$ These findings may be explained by primary RV myocardial involvement, but alternatively the presence of $\mathrm{PAH}$, resulting from extensive pulmonary sarcoidosis, has been considered a possible cause of RV fibrosis. $(\mathbf{3 1 8 , 3 3 0 )}$ (Figures $\mathbf{7 , 9 , 1 8 , 2 0 )}$ Smedema et al compared the LGE distribution in 30 CS patients with subsequent patients with ischemic infarct scar in blinded fashion, and were able to differentiate between these conditions in $80 \%$ of cases. (330) In a number of infarct patients who underwent primary PCI the LGE was distributed in an atypical pattern more suggestive of NICM. (328) These findings suggest that DECMR is helpful in the non-invasive differentiation between CS and ischemic scar. In conclusion, the presence of sole mid-layer, epicardial and patchy three layer LGE with a non-vascular distribution, in patients with extra-cardiac sarcoidosis suggests cardiac involvement, and differentiates these patients from patients with coronary artery disease and a variety of other NICMs. (330)

\subsection{Prognostic value of DECMR - CMR as part of risk stratification}

Symptomatic CS carries a poor prognosis unless adequately managed with medical therapy, cardiac implantable devices (CID), arrhythmia ablation, or, in advanced irreversible heart failure, heart transplantation. (11,12,95,99,204,230,238,328,329) Apart from symptomatic presentation (CCF, syncope, palpitations), ECG (late potentials, Sylvester QRS score, nsVT or sVT on ambulatory rhythm monitoring), inducible sVT/VF with PES, impaired global 
longitudinal LV strain, impaired systolic LV or RV functions (cardiac ultrasound, CMR, MDCT, myocardial perfusion scintigraphy (MPS)/SPECT), LV and/or RV LGE on DECMR, and LV and/or RV FDG uptake with PET are predictors of admission for CCF, PM implantation and/or cardiac death. (11,14,29,29,35,5052,84,100,115,116,135,136,201, 226,301, 330-338) The presence, amount, transmural extent, LV and particular RV LGE carry strong independent risk of adverse outcome. Table 9 presents the baseline data of the 9 DECMR studies included in our meta-analysis, which have provided prognostic data. $(30,133,177,204,289,298,339-341)$ We conducted our meta-analysis based on the methods published by Hulten et al. (134) Since Hulten's original publication, two additional studies were published, which fulfilled the inclusion criteria. $(30,341)$ Tables 10 and 11 provide detail on the prognostic relevance of any (LV, RV, biventricular) LGE or RV LGE for sVT, admission for CCF, PM implantation for high-grade AVB, cardiovascular death, or all cause death. Figures 29-36 depict the respective pooled relative risk (RR) of any myocardial LGE or RV LGE for these adverse events. Myocardial LGE predicts future cardiovascular adverse events and death. RV LGE, inflammation and impaired systolic function have previously been associated with adverse outcome, particularly ventricular tachyarrhythmias. (95,133,204, 230,238,301) Biventricular LGE at presentation may be the strongest, independent predictor of adverse outcome during long-term follow up. (30) The significance of small asymptomatic myocardial scar needs further study. Several prospective studies have demonstrated a favorable long-term outcome in NICM, including CS, when LV scar was $<8 \%$ of LV mass. $\mathbf{( 3 0 , 1 0 0 , 3 3 3 , 3 3 6 , 3 3 9 , 3 5 2 ) ~ L G E ~ i s ~ t h e ~ u n d e r l y i n g ~ p a t h o - ~}$ physiological substrate of myocardial dysfunction, conduction disease and ventricular arrhythmias. DECMR allows for direct quantification of focal scar, and as expected this parameter out-performs secondary markers of myocardial disease such as LVEDV and LVEF.

\subsection{The relevance of screening for subclinical cardiac sarcoidosis}

Approximately $5-10 \%$ of sarcoidosis patients present with symptoms of CS. Although routine DECMR has revealed small asymptomatic scar in $20-25 \%$ of sarcoidosis patients, the significance of these findings is uncertain. $(\mathbf{3 0 , 9 9 , 3 4 3 )}$ The prognosis of small asymptomatic scar seems favorable. Current guidelines advise against routine evaluation with $\mathrm{PET} / \mathrm{CT}$ and/or CMR in sarcoidosis patients without cardiovascular symptoms and normal ECGs and/or CUS, despite the fact that myocardial scar may remain undetected with this basic baseline assessment. $(\mathbf{1 1}, \mathbf{4 6 , 2 5 2 , 2 5 4 , 2 7 8 , 3 4 3 )}$ There is currently no consensus concerning the intervals of assessment with advanced imaging technology such as PET/CT and CMR in symptomatic patients.

\subsection{DECMR assists in clinical management of cardiac sarcoidosis}

DECMR guides the interventionalist in obtaining representative EMBs by the preprocedural delineation of the localization and extent of granulomatous inflammation. Preproce- 
dural imaging will customize the approach to endocardial, epicardial, RV or LV. $(\mathbf{7 7 , 8 1 )}$ In patients with newly diagnosed AVB and/or myocardial dysfunction DECMR will help diagnose and manage CS as the potential reversible cause. (7,12,13,222,263,332,344) (Figures 6,19) In PM patients with progressive systolic LV dysfunction DECMR helps differentiate progressive CS from pacing-induced cardiomyopathy. (345) In patients with LBBB, LV dyssynchrony and systolic dysfunction DECMR will help predict a favorable response to biventricular pacing, by localizing and quantifying LV scar. $(346,347)$ DECMR assists the electrophysiologist in identifying and delineating the arrhythmic substrate and determine the optimal, endocardial, epicardial or hybrid, approach needed for successful ablation. (226,348-354) DECMR provides crucial information concerning the transmural distribution of scar which surface mapping cannot provide. $(351,352,354)$ The amount, localization and distribution of LGE have been associated with the risk of VT and SCD. Midmyocardial LGE involving $25-75 \%$ of the wall thickness predicts inducible VT at PES. $(30,100,351,353)$ Asymptomatic CS patients with limited LGE $(<8 \%)$, preserved LVEF and negative PES have a favorable prognosis $(30,100)$ DECMR provides post-ablation feedback concerning the depth and distribution of the lesions are linked with ablation failure. Eventually real-time DECMR guided endomyocardial mapping and ablation will increase procedural success rates. $(\mathbf{3 5 4 , 3 5 5 , 3 5 6 , 3 5 7 )}$ In addition to PET DECMR will help optimize immune-suppressive treatment for CS, and risk-stratify i.e. when to implant an ICD by evaluating systolic LV and RV functions, defining and quantifying myocardial scar. $(11,30,293,332,358)$

\subsection{Improved outcomes with modern management}

Long-term outcomes in pts with CS have remarkably improved due to accurate diagnostic imaging, modern medical heart failure treatment, the introduction of CIDs, arrhythmia ablation and transplantation in selected patients. (11,12,25,27,28,98,230,331,335,336,361) Five-year survival rates have improved from $41 \%$ to current 5 and 10 -year survival rates of over $90 \%$. (30,98,263,328,329,358,359-361) Current annual mortality rates range from 0 $4.2 / 100$ pts compared to $7.5-12 / 100$ pts as previously reported by Yazaki (2001) and Fleming (1974). (20,30,48,359,362)

\subsection{Conclusions}

DECMR has become an important modality in the diagnosis, risk stratification and management of CS. In addition to accurately and reproducibly quantifying myocardial structure and functions, DECMR characterizes myocardial inflammation, infiltration, focal and interstitial fibrosis, thereby guiding management, resulting in improved outcomes. 


\subsection{New and future applications of CMR in the management of cardiac sarcoidosis (Table 12)}

Ultra-high field CMR and compressed-sensing techniques will shorten scan times, improve image quality and spatial resolution. (212) Mapping multiple relaxation times simultaneously will similarly save time and remove confounders introduced by the interaction of relaxation times. Three-dimensional mapping allows for more complete characterization of disease distribution, so-called CMR fingerprinting of the myocardium, which implies the generation of structural, mechanical, functional as well as biochemical information. $(210,212,367)$ DECMR is not accurate in detecting and quantifying RV scar. Additional RV CMR strain imaging will enhance the diagnostic accuracy for RV sarcoidosis, and may assist in increasing the success rate of RV ablation procedures. (371) Detailed information concerning focal scar and diffuse biventricular interstitial edema, fibrosis, as well as segmental and global systolic functions and myocardial strain, will improve risk stratification and help determine which patients will benefit from ICD implantation. Future, prospective research will determine whether management decisions based on parametric mapping will improve outcomes. Hybrid PET/CMR imaging which combines the advantages of DECMR (image resolution, scar imaging, high specificity) with those of PET (high sensitivity for inflammation), will reduce examination time and cost, while individualized treatment based on serial investigations may help improve outcome. (125,126,235,278,377-380) Recent advances have made real-time CMR guided EMB in patients with suspected isolated CS and ablations of (supra)ventricular arrhythmias a reality. $(368,370,371)$

\subsection{Acknowledgements}

We gratefully acknowledge Alfred Musekiwa's expert statistical support, as well as GeertJan Zonneveld, Michael Wyeth, and Stella van Schalkwijk's skill in drawing the figures. 


\section{References}

1. Valeyre D, Prasse A, Nunes H, Uzunhan Y, Brillet PY, Müller-Quernheim J. Sarcoidosis. Lancet 2014;383:1155-1167. doi: 10.1016/S0140-6736(13)60680-7.

2. Constabel U, Hunninghake GW. ATS/ERS/WASOG statement on sarcoidosis: Sarcoidosis Statement Committee, American Thoracic Society, European Respiratory Society, World Association for Sarcoidosis and Other Granulomatous Disorders. Eur Respir J 1999;14(4):735-737.

3. Statement on sarcoidosis. Joint Statement of the American Thoracic Society (ATS), European Respiratory Society (ERS) and World Association of Sarcoidosis and Other Granulomatous Disorders (WASOG) adopted by the ATS Board of Directors and by the ERS Executive Committee, February 1999. Am J Resp Crit Care Med 1999;160(2):736-755.

4. Crouser ED, Amin EN. Severe sarcoidosis phenotypes: An occupational hazard? Chest 2016;150(2):263-265. doi: 10.1016/j.chest.2016.02.663.

5. Chen ES, Moller DR. Etiology of sarcoidosis. Clin Chest Med 2008;29:365-377. doi: 10.1016/j.ccm.2008.03.011.

6. Asakawa N, Uchida K, Sakakibara M, Omote K, Noguchi K, Tokuda Y, Kamiya K, Hatanaka KC, Matsuno Y, Yamada S, Asakawa K, Fukasawa Y, Nagai T, Anzai T, Ikeda Y, Ishibashi-Ueda H, Hirota M, Orii M, Akasaka T, Uto K, Shingu Y, Matsui Y, Morimoto S, Tsutsui H, Eishi Y. Immuno-histochemical identification of Propionibacterium acnes in granuloma and inflammatory cells of myocardial tissues obtained from cardiac sarcoidosis patients. PLos One 2017;12(7):e0179980. doi.org/10.1371/journal.pone.0179980

7. Kandolin R. Cardiac Sarcoidosis and Giant Cell Myocarditis in Finland. Academic Dissertation, University of Helsinki Heart Centre 2015. ISBN 978-951-51-1769-4.

8. Rosen Y. Pathology of sarcoidosis. Sem Resp Crit Care Med 2007;28(1):36-52.

9. Hutchinson I. Cases of Mortimer's malady, a form of lupus pernio. Arch Surg 1898;9:307-315.

10. Bernstein M, Konzleman FW, Sidlick DM. Boeck's sarcoid - report on a case with visceral involvement. Arch Intern Med 1929;44:721-734.

11. Birnie DH, Sauer WH, Bogun F, Cooper JM, Culver DA, Duvernoy CS, Judson MA, Kron J, Mehta D, Cosedis Nielsen J, Patel AR, Ohe T, Raatikainen P, Soejima K. HRS Expert consensus statement on the diagnosis and management of arrhythmias associated with cardiac sarcoidosis. Heart Rhythm 2014;11(7):13051323. doi: 10.1016/j.hrthm.2014.03.043.

12. Birnie DH, Kandolin R, Nery PB, Kupari M. Cardiac manifestations of sarcoidosis: diagnosis and management. Eur Heart J. 2017;38(35):2663-2770. doi:10.1093/eurheartj/ehw328.

13. Birnie DH, Nery PB, Ha AC, Beanlands RS. Cardiac sarcoidosis. J Am Coll Cardiol. 2016;26;68(4):411-21. doi: 10.1016/j.jacc.2016.03.605.

14. Mehta D, Lubitz SA, Frankel Z, Wisnivesky JP, Einstein AJ, Goldman M, Machac J, Teirstein A. Cardiac involvement in patients with sarcoidosis: diagnostic and prognostic value of outpatient testing. Chest 2008;133(6):1426-35. doi: 10.1378/chest.07-2784.

15. Lynch JP, Hwang J, Bradfield J, Fishbein M, Shivkumar K, Tung R. Cardiac involvement in sarcoidosis: evolving concepts in diagnosis and treatment. Sem Resp Crit Care Med 2014;35:372-390. doi: 10.1055/s0034-1376889.

16. Roberts WC, McAllister HA Jr, Ferrans VJ. Sarcoidosis of the heart: a clinico-pathological study of 35 necropsy patients (group 1) and review of 78 previously described necropsy patients (group II). Am J Med 1977;63:86-108.

17. Silverman KJ, Hutchins GM, Bulkley BH. Cardiac sarcoid: a clinico-pathologic study of 84 unselected patients with systemic sarcoidosis. Circulation 1979;58:1204-1211.

18. Tavora F, Creswell N, Li L, Ripple M, Solomon C, Burke A. Comparison of necropsy findings in patients with sarcoidosis dying suddenly from cardiac sarcoidosis versus dying suddenly from other causes. Am J Cardiol 2009;104:571-77. doi: 10.1016/j.amjcard.2009.03.068.

19. Gozo E, Cosnow I, Cohen H, Okun L. Critical review. The heart in sarcoidosis. Chest 1971:60(4):379-388.

20. Matsui Y, Iwai K, Tachibana T, Fruie T, Shigematsu N, Izumi T, Homma AH, Mikami R, Hongo O, Hiraga Y, Yamamoto M. Clinico-pathological study on fatal myocardial sarcoidosis. Ann NY Acad Sci 1976;278:455-469.

21. Lagana SM, Parwani AV, Nichols LC. Cardiac sarcoidosis: A pathology-focused review. Arch Pathol Lab Med 2010;134:1039-1049. doi: 10.1043/2009-0274-RA.1.1 
22. Branson JH, Park JH. Sarcoidosis - Hepatic involvement: presentation of a case with fatal liver involvement including autopsy findings and review of the evidence for sarcoid involvement in the liver as found in the literature. Ann Int Med 1954;40:111-145.

23. Iwai K, Sekigichi M, Hosada Y, DeRemeree RA, Tazelaar HD, Sharma OP, Maheshwari A, Nohuchi TI. Racial difference in cardiac sarcoidosis: Incidence observed at autopsy. Sarcoidosis 1994:11(1):26-31.

24. Cain MA, Metzl MD, Patel AR, Addetia K, Spencer KT, Sweiss NJ, Beshai JF. Cardiac sarcoidosis detected by late gadolinium enhancement and prevalence of atrial arrhythmias. Am J Cardiol 2014;113(9):1556-1560. doi: 10.1016/j.amjcard.2014.01.434.

25. Ward EV, Nazari J, Edelman RR. Coronary artery vasculitis as a presentation of cardiac sarcoidosis. Circulation 2012;125:e344-e346. doi: 10.1161/CIRCULATIONAHA.110.990747.

26. Lam CSP, Tolep KA, Metke MP, Glockner J, Cooper LT. Coronary sarcoidosis presenting as acute coronary syndrome. Clin Cardiol 2009;32:68-71. doi: 10.1002/clc.20381.

27. Patel D, Xie K, Sweiss NJ, Lu Y. Sarcoid pericarditis and large vessel vasculitis detected on FDG PET/CT. Clin Nucl Med 2016;41(8):661-663. doi: 10.1097/RLU.0000000000001267.

28. Halushka MK, Yuh DD, Russell SD. Right ventricle-dominant cardiac sarcoidosis with sparing of the left ventricle. J Heart Lung Transplant 2006;28:479-82.

29. Smedema JP, van Geuns RJ, Ector J, Ainslie G, Heidbuchel H, Crijns HJGM. Right ventricular involvement in cardiac sarcoidosis demonstrated with cardiac magnetic resonance. ESC Heart Failure 2017;4(4):535-544. doi: 10.1002/ehf2.12166.

30. Smedema JP, van Geuns RJ, Ector J, Ainslie G, Heidbuchel H, Crijns HJGM. Right ventricular involvement and the extent of left ventricular enhancement with magnetic resonance predict adverse outcome in pulmonary sarcoidosis. ESC Heart Failure 2018; (1):157-171. doi: 10.1002/ehf2.12201.

31. Lønborg J, Ward M, Gill A, Grieve SM, Figtree GA. Utility of cardiac magnetic resonance in assessing rightsided heart failure in sarcoidosis. BMC Medical Imaging 2013;13:2. doi: 10.1186/1471-2342-13-2.

32. Vakil K, Minami E, Fishbein DP. Right ventricular sarcoidosis: is it time for updated diagnostic criteria? Tex Heart Inst J 2014;41(2):203-207. doi: 10.14503/THIJ-12-3086.

33. Uchida M, Shinohara T, Takahashi N, Saikawa T. Interventricular septal mass in a patient with cardiac sarcoidosis. J Cardiovasc Electrophys 2012;23:433-435. doi: 10.1111/j.1540-8167.2011.02227.

34. Pedrotti P, Ammirati E, Bonacina E, Roghi A. Ventricular aneurysms in cardiac sarcoidosis: From pathophysiology to surgical treatment through a clinical case presenting with ventricular arrhythmias. Int J Cardiol 2015;186;294-296. doi: 10.1016/j.ijcard.2015.03.256.

35. Miyazawa K, Yoshikawa T, Takamisawa I, Mahara K, Inoue K, Iguchi N, Takayama M, Umemura J, Sumiyoshi T, Tomoike H. Presence of ventricular aneurysm predicts poor clinical outcomes in patients with cardiac sarcoidosis. Int J Cardiol 2014;177(2):720-722. doi: 10.1061/j.ijcard.2014.10.024.

36. Smedema JP. Tissue Doppler Imaging in cardiac sarcoidosis. Eur J Echocardiogr 2008;9(4):579-580. doi: 10.1093/ejechocard/jen073.

37. Sato Y, Matsumoto N, Kunimasa T, Imai S, Matsuo S, Nakano Y, Yoda S, Kunimoto S, Saito S, Hirayama A. Multiple involvements of cardiac sarcoidosis in both left and right ventricles and papillary muscles detected by delayed-enhanced magnetic resonance imaging. Int J Cardiol 2008;130:288.

38. Yang Y, Safka K, Graham JJ, Roifman I, Zia MI, Wright GA, Balter M, Dick AJ, Conelly KA. Correlation of late gadolinium enhancement MRI and quantitative T2 measurement in cardiac sarcoidosis. J Magn Res Imag 2014;39(3):609-616. doi: 10.1002/jmri.24196.

39. Garrett J, O’Neill H, Blake S. Constrictive pericarditis associated with sarcoidosis. Am Heart J 1984;107:394.

40. Darda S, Zughaib ME, Alexander PB, Machado CE, David SW, Saba S. Cardiac sarcoidosis presenting as constrictive pericarditis. Tex Inst Heart J 2014;41(3):319-323. doi: 10.14503/THIJ-13-3208.

41. Marie I, Lahaxe L, Guilbert M, Dacher JN, Levesque H. Pericarditis revealing a relapse of sarcoidosis. South Med J 2009;102(12):1275-1277. doi: 10.1097/SMJ.0b013e3181bcaa0f.

42. Zelcer AA, LeJemtel TH, Jones J, Stahl J. Pericardial tamponade in sarcoidosis. Can J Cardiol 1987;3(1):1213.

43. Rybicki BA, Ianuzzi MC. Epidemiology of sarcoidosis: Recent advances and future prospects. Sem Resp Crit Care Med 2007;28(1):22-34.

44. Perry A, Vuitch F. Causes of death in patients with sarcoidosis: a morphologic study of 38 autopsies with clinico-pathologic correlations. Arch Pathol Lab Med 1995;119:167-72.

45. Chiles C, Adams GW, Ravin CE. Radiographic manifestations of cardiac sarcoid. AJR Am J Roentgenol 1985;145(4):711-714. 
46. Slart RHJA, Glaudemans AWJM, Lancellotti P, Hyafil F, Blankstein R, Schwartz RG, Jaber WA, Russell R, Gimelli A, Rouzet F, Hacker, Gheysens O, Plein S, Miller EJ, Dorbala S, Donal E. A joint procedural position statement on imaging in cardiac sarcoidosis: From the Cardiovascular and Inflammation \& Infection Committees of the European Association of Nuclear Medicine, the European Association of Cardiovascular Imaging, and the American Society of Nuclear Cardiology. J Nucl Cardiol 2018;25(1):298-319. doi: $10.1007 /$ s12350-017-1043-4.

47. Lewin RF, Mor R, Spitzer S, Arditti A, Hellman C, Agmon J. Echocardiographic evaluation of patents with systemic sarcoidosis. Am Heart J 1985;110:116-22.

48. Yazaki Y, Isobe M, Hiroe M, Morimoto A, Hiramitsu, Nakano T, Izumi T, Sekiguchi M. Prognostic determinants of long-term survival in Japanese patients with cardiac sarcoidosis treated with prednisone. Am J Cardiol 2001;88:1006-1010.

49. Burtow DJ, Tajik AJ, Baily KR, DeRemee RA, Taliercio CP. Two-dimensional echocardiographic findings in systemic sarcoidosis. Am J Cardiol 1989;63:478-482.

50. Joyce E, Nienaber MK, Katsanos S, Debonnaire P, Kamperidis V, Bax JJ, Taube C, Delgado V, Ajmone Marsan M. Subclinical left ventricular dysfunction by echocardiographic speckle- tracking strain analysis relates to outcomes in sarcoidosis. Eur J Heart Fail 2015;28:1-39e14. doi: 10.1002/ejhf.205.

51. Joyce E, Kamperidis V, Nienaber MK, Kansans S, Debonair P, Schally MJ, Taube C, Box JJ, Delgado V, Ajmone Marsan M. Prevalence and correlates of early right ventricular dysfunction in sarcoidosis and its association with outcome. J Am Soc Echocardiogr 2016;29(9):871-878. doi: 10.1016/j.echo.2016.06.001.

52. Sperry BW, Ibrahim A, Negishi K, Negishi T, Patel P, Popovic ZB, Culver D, Brunken R, Marwick TH, Tamarappoo B. Incremental prognostic value of global longitudinal strain and 18-F Fludeoxyglucose Positon Emission Tomography in patients with systemic sarcoidosis. Am J Cardiol 2017;119:1663-1669. doi: 10.1016/j.amjcard.2017.02.010.

53. Ngano N, Nagai T, Sugano Y, Morita Y, Asumi Y, Aiba T, Kanzaki K, Kusano K, Noguchi T, Yasuda S, Ogawa $\mathrm{H}$, Anzai T. Association between basal thinning of interventricular septum and adverse long-term clinical outcomes in patients with cardiac sarcoidosis. Circ J 2015;79(7):1601-1618. doi: 10.1253/circj.CJ-141217.

54. Fahy GJ, Marwick T, McCreery CJ, Quigley PJ, Maurer BJ. Doppler echocardiographic detection of left ventricular diastolic dysfunction in patients with pulmonary sarcoidosis. Chest 1996;109(1):62-66.

55. Felekos I, Aggeli C, Gialafos E, Kouranos V, Rapti A, Sfikakis P, Koulouris N, Tousoulis D. Global longitudinal strain and long-term outcomes in asymptomaic extra-cardiac sarcoid patients with no apparent cardiovascular disease. Echocardiography 2018;35(6):804-808. doi: 10.1111/echo.13846.

56. Murtagh G, Laffin LJ, Patel KV, Patel AV, Bonham CA, Laffin LJ, Hogarth DK, Medvedofsky D, Lang RM, Patel AR. Improved detection of myocardial damage in sarcoidosis using longitudinal strain in patients with preserved left ventricular ejection fraction. Echocardiography 2016;33:1344-1352. doi: 10.1111/echo.13281.

57. Kul S, Ozcelik HK, Uyarel H, Karakus G, Guvenc TS, Yalcinsoy M, Asoglu E, Kemik AS, Tasal A, Gungor S, Karaarslan E, Kart L, Goktekin O. Diagnostic value of strain echocardiography, galectin-3, and tenascin-C levels for the identification of patients with pulmonary and cardiac sarcoidosis. Lung 2014;192(4):533-542. doi: 10.1007/s00408-014-9586-5.

58. Kiko T, Yoshihisa A, Kanno Y, Yokokawa T, Abe S, Miyata-Tatsumi M, Misaka T, Oikawa M, Kobayashi A, Ishida T, Takeishi Y. Int Heart J 2018 August 11. doi: 10.1536/ihj.17-695. [Epub ahead of print]

59. Baba Y, Kubo T, Kitaoka H, Okawa M, Yamanaka S, Kawada Y, Yamasaki N, Matsumura Y, Furuno T, Sugiura T, Doi YL. Usefulness of high-sensitive cardiac troponin T for evaluating the activity of cardiac sarcoidosis. Int Heart J 2012;53(5):287-92. doi:

60. Lieberman J. The specificity and nature of serum-angiotensin-converting enzyme (serum ACE) elevations in sarcoidosis. Ann N Y Acad Sci 1976;278:488-497.

61. Fuse K, Kodama M, Okura Y, Ito M, Aoki Y, Hirono S, Kato K, Hanawa H, Aizawa Y. Levels of serum interleukin-10 reflect disease activity in patients with cardiac sarcoidosis. Jpn Circ J 2000;64(10):755-759.

62. Miyoshi S, Hamada H, Kadowaki T, Hamaguchi N, Ito R, Irifune K, Higaki J. Comparative evaluation of serum markers in pulmonary sarcoidosis. Chest 2010;137(6):1391-1397. doi: 10.1378/chest.09-1975.

63. Kandolin R, Lehtonen J, Airaksinen J, Vihinen T, Miettinen H, Kaikkonen K, Haataja P, Kertola T, Kupari M. Usefulness of cardiac troponins as markers of early treatment response in cardiac sarcoidosis. Am J Cardiol 2015;116:960-964. doi: 10.1016/j.amjcard.2015.06.021. 
64. Tanada Y, Sato Y, Sawa T, Fujiwara H, Takatsu Y. Serial measurement of high-sensitivity cardiac troponin T and N-terminal proB-type natriuretic peptide in a patient presenting with cardiac sarcoidosis. Intern Med 2012;51:3379-3381.

65. Handa T, Nagai S, Ueda S, Chin K, Ito Y, Watanabe K, Tanizawa K, Tamaya M, Mishima M, Izumi T. Significance of plasma NT-pro-BNP levels as a biomarker in the assessment of cardiac involvement and pulmonary hypertension in patients with sarcoidosis. Sarcoidosis Vasc Diffuse Lung Dis 2010;27:27-35.

66. Date T, Shinozaki T, Yamakawa M, Taniguchi I, Suda A, Hara H, Yamane T, Komukai K, Sugimoto K, Mochizuki S. Elevated plasma brain natriuretic peptide level in cardiac sarcoidosis patients with preserved ejection fraction. Cardiology 2007;107:277-280. doi: 10.1159/000095518.

67. Crouser ED, Hamzeh NY, Maier LA, Julian MW, Gillespie M, Rahman M, Baxter D, Wu X, Nana-sinkam SP, Wang K. Exosomal MicroRNA for the detection of cardiac sarcoidosis. Am J Resp Crit Care Med 2017;196(7):931-934. doi: 10.1164/rccm.201611-2183LE.

68. Fujiwara W, Kato Y, Hayashi M, Sugishita Y, Okumura S, Yoshinaga M, Ishiguro T, Yamada R, Ueda S, Harada M, Naruse H, Ishii J, Ozaki Y, Izawa H. Serum microRNA-126 and -223 as a new generation biomarkers for sarcoidosis in patients with heart failure. J Cardiol 2018;72:452-457. doi: 10.1016/j.jjcc.2018.06.004.

69. Blaschke E, Eklund A. Sarcoidosis and neopterin. Am Rev Respir Dis 1990;141(5 Pt1):1379.

70. Lacronique J, Auzeby A, Valeyre D, Traore BM, Barbosa ML, Soler P, Choudat D, Battesti JP, Touitou Y, Marsac J. Urinary neopterin in pulmonary sarcoidosis. Relationship to clinical and biologic assessment of the disease. Am Rev Respir Dis 1989;141(5 Pt1):1379.

71. Vorselaars AD, van Moorsel CH, Zanen P, Ruven HJ, Claessen AM, van Velzen-Blad H, Grutters JC. ACE and sIL-2R correlate with lungfunction improvement in sarcoidosis during methotrexate therapy. Respir Med 2015;109:279-285. doi: 10.1016/j.rmed.2014.11.009.

72. Myoren T, Kobayashi S, Oda S, Nanno T, Ishiguchi H, Murakami W, Okuda S, Okada M, Takamura G, Suga K, Matzuzaki M, Yano M. An oxidative stress biomarker, urinary 8-Hydroxy-2'-Deoxyguanosine, predicts cardiovascular-related death after steroid therapy for patients with active cardiac sarcoidosis. Int J Cardiol 2016;2012:206-213. doi: 10.1016/j.ijcard.2016.03.003.

73. Ishiguchi H, Kobayashi S, Myoren T, Kohno M, Nanno T, Murakami W, Oda S, Oishi K, Okuda S, Okada M, Suga K, Yano M. Urinary 8-Hydroxy-2'-Deoxyguanosine as a myocardial oxidative stress marker is associated with ventricular tachycardia in patients with active sarcoidosis. Circ Cardiovasc Imaging 2017;10(12). pii: e006764. doi: 10.1161/circimaging.117.006764.

74. Ardehali H, Howard DL, Hariri A, Quasim A, Hare JM, Baughman KL, Kasper EK. A positive endomyocardial biopsy result for sarcoid is associated with poor prognosis in patients with initially unexplained cardiomyopathy. Am Heart J 2005;150(3):459-463. doi: 10.1016/j.ahj.2004.10.006.

75. Uemura A, Morimoto S, Hiramitsu S, Kato Y, Ito T, Hishida H. Histologic diagnostic rate of cardiac sarcoidosis: evaluation of endomyocardial biopsies. Am Heart J 1999;138:299-302.

76. Nery P, Keren A, Healey J, Leung E, Beanlands RS, Birnie DH. Isolated cardiac sarcoidosis: establising the diagnosis with electroanatomic mapping-guided endomyocardial biopsy. Can J Cardiol 2013;29:1015.e11015.e3. doi: 10.1016/j.cjca.2012.09.009.

77. Borchert B, Lawrenz T, Bartelsmeier M, Röthemeyer S, Kuhn H, Stellbrink C. Utility of endomyocardial biopsy guided by delayed enhancement areas on magnetic resonance imaging in the diagnosis of cardiac sarcoidosis. Clin Res Cardiol 2007;96(10):759-762. doi: 10.1007/s00392-007-0557-1.

78. Honda Y, Nagai T, Ikeda Y, Sakakibara M, Asakawa N, Nagano N, Nakai M, Nishimura K, Sugano Y, Ohta-Ogo K, Asaumi Y, Aiba T, Kanzaki H, Kusano K, Noguchi T, Yasuda S, Tsutsui H, Ishibashi-Ueda H, Anzai T. Myocardial immune-competent cells and macrophage phenotypes as histo-pathological surrogates for diagnosis of cardiac sarcoidosis in Japanese. J Am Heart Assoc 2016;5(11). Pii:e004019. doi: 10.1161/JAHA.116.004019.

79. Muser D, Santangeli P, Liang JJ, Castro SA, Magnani S, Hayashi T, Garcia FC, Frankel DS, Dixit S, Zado ES, Lin D, Desjardins B, Callans DJ, Alavi A, Marchlinski FE. Characterization of the electroanatomic substrate in cardiac sarcoidosis: Correlation with imaging findings of scar and inflammation. JACC Clin Electrophysiol 2018;4(3):291-303. doi: 10.1016/j.jacep.2017.09.175.

80. Vaidya VR, Abudan AA, Vasudevan K, Shantha G, Cooper LT, Kapa S, Noseworthy PA, Cha YM, Asirvatham SJ, Deskmukh AJ. The efficacy and safety of electroanatomic mapping-guided endomyocardial biopsy: a systematic review. J Interv Card Electrophysiol 2018 Jul 12. doi: 10.1007/s10840-018-0410-7. [Epub ahead of print] PMID: 30003460 
81. Bhimaraj A, Trachtenberg B, Valderrabáno M. Robotically assisted left ventricular biopsy to diagnose cardiac sarcoidosis: A multidisciplinary innovation leading to first-in-human case. Circ Heart Fail 2018;11(3):e004627. doi: 10.1161/CIRCHEARTFAILURE.117.004627.

82. Takaya Y, Kusano KF, Nakamura K, Ito H. Outcomes in patients with high-degree atrioventricular block as the initial manifestation of cardiac sarcoidosis. Am J Cardiol 2015;115:505-509. doi: 10.1016/j.amjcard.2014.11.028.

83. Freeman AM, Curran-Everett D, Weinberger HD, Fenster BE, Buckner JK, Gottschall EB, WH Sauer, Maier LA, Hamzeh NY. Predictors of cardiac sarcoidosis using commonly available cardiac studies. Am J Cardiol 2013;112:280-285. doi: 10.1016/j.amjcard.2013.03.027.

84. Sobue Y, Harada M, Kohsikawa M, Ischikawa T, Yamamoto M, Okuda K, Kato Y, Sarai M, Watanabe A, Ozaki Y. QRS-based assessment of myocardial damage and adverse events associated with cardiac sarcoidosis. Heart Rhythm 2015;12:2499-2507. doi: 10.1016/j.hrthm.2015.09.008.

85. Homsi M, Alsayed L, Safadi B, Mahenthiran J, Das MK. Fragmented QRS complexes on the 12-lead ECG: A marker of cardiac sarcoidosis as detected by gadolinium cardiac magnetic resonance imaging. Annals of Noninvasive Electrocardiology 2009;14(4):319. doi: 10.1111/j.1542-474X2009.00320.x.

86. Sipilä K, Tuominen H, Haarala A, Tikkakoski A, Kähönen M, Nikus K. Novel ECG parameters are strongly associated with inflammatory ${ }^{18} \mathrm{~F}-\mathrm{FDG}$-PET findings in patients with suspected cardiac sarcoidosis. Intern J Cardiol 2017;249:454-460. doi:10.1016/j.ijcard.2017.07.027.

87. Matsumoto S, Hirayama Y, Saitoh H, Ino T, Miyauchi Y, Iwasaki Y, Yasutake M, Seino Y, Atarashi H, Katoh T, Mizuno K. Non-invasive diagnosis of cardiac sarcoidosis using microvolt T-wave alternans. Int Heart J 2009;50:731-739.

88. Yodogawa K, Seino Y, Ohara T, Takayama H, Kobayashi Y, Katoh T, Takano T. Non-inavasive detection of latent cardiac conduction abnormalities in patients with pulmonary sarcoidosis. Circ J 2007;71:540-545.

89. Uyarel H, Uslu N, Okmen E, Tartan Z, Kasikcioglu H, Dahi SU, Cam N. QT dispersion in cardiac sarcoidosis. Chest 2005;128:2619-2625. doi: 10.1378/chest.128.4.2619.

90. Tanaka Y, Konno T, Yoshida S, Tsuda T, Sakata K, Furusho H, Takamura M, Yoshimura K, Yamagishi M, Hayashi K. T wave amplitude in lead aVR as a novel diagnostic marker of cardiac sarcoidosis. Heart Vessels 2017;32(3):352-358. doi: 10.1007/s00380-016-0881-3.

91. Thunell M, Bjerle P, Karp K, Stjernberg N. Exercise test in patients with sarcoidosis. The importance of repolarization disturbances. Acta Med Scand 1988;223:69-73.

92. Gibbons WJ, Levy RD, Nava S, Malcolm I, Marin JM, Tardif C, Magder S, Lisbona R, Cosio MG. Subclinical cardiac dysfunction in sarcoidosis. Chest 1991;100(1):44-50.

93. Rubinstein I, Fisman EZ, Rosenblum Y, Pines A, Shiner RJ, Ben-Ari E, Baum GL, Kellermann JJ. Leftventricular exercise echocardiographic abnormalities in patients with sarcoidosis without ischemic heart disease. Isr J Med Sci 1986;22(12):865-872.

94. Suzuki T, Kanda T, Kubota S, Imai S, Murata K. Holter monitoring as a non-invasive indicator of cardiac involvement in sarcoidosis. Chest 1994;106:1021-1024.

95. Kron J, Sauer W, Mueller G, Schuller J, Bogun F, Sarsam S, Rosenfeld L, Mitiku TY, Cooper JM, Mehta D, Greenspon AJ, Ortman M, Delurgio DB, Valadri R, Narasimhan C, Swapna N, Singh JP, Danik S, Markowitz SM, Almquist AK, Krahn AD, Wolfe LG, Feinstein S, Ellenbogen KA, Crawford T. Outcomes of patients with definite and suspected isolated cardiac sarcoidosis treated with an implantable cardiac defibrillator. J Interv Card Electrophysiol 2015;43(1):55-64. doi: 10.1007/s108040-015-9978-3.

96. Betensky BP, Tschabrunn CM, Zado ES, Goldberg LR, Marchlinski FE, Garcia FC, Cooper JM. Long-term follow-up of patients with cardiac sarcoidosis and implantable cardioverter- defibrillators. Heart Rhythm 2012;9(6):884-891. doi: 10.1016/j.hrthm.2012.02.010.

97. Schuller JL, Lowery CM, Zipse M, Aleong RG, Varosy PD, Weinberger HD, Sauer WH. Diagnostic utility of signal-averaged ECG for detection of cardiac sarcoidosis. Ann Noninvasive Electrocardiol 2011;16:70-76. doi: 10.1111/j.1542-474X.2010.00411.x.

98. Mohsen A, Jimenez A, Hood RE, Dickfeld T, Saliaris A, Shorofsky S, Saba MM. Cardiac sarcoidosis: electrophysiological outcomes on long-term follow-up and the role of the implantable cardioverter-defibrillator. J Cardiovasc Electrophys 2014;25(2):171-176. doi: 10.1111/jce.12302.

99. Smedema JP, Snoep G, van Kroonenburgh MP, van Geuns RJ, Dassen WR, Gorgels AP, Crijns HJGM. Cardiac involvement in patients with pulmonary sarcoidosis assessed at two University Medical Centres in The Netherlands. Chest 2005;128(1):30-35. doi: 10.1378/chest.128.1.30. 
100. Mehta D, Mori N, Goldberg SH, Lubitz S, Wisnivesky JP, Teirstein A. Primary prevention of sudden cardiac death in silent cardiac sarcoidosis: role of programmed ventricular stimulation. Circ Arrhythm Electrophysiol 2011;4(1):43-48. doi: 10.1161/CIRCEP.110.958322.

101. Aizer A, Stern EH, Gomes JA, Teirstein AS, Eckart RE, Mehta D. Usefulness of programmed ventricular stimulation in predicting future arrhythmic events in patients with cardiac sarcoidosis. Am J Cardiol 2005;96:276-282. doi: 10.1016/j.amjcard.2005.03.059.

102. Winters SL, Cohen M, Greenberg S, Stein B, Curwin J, Pe E, Gomes JA. Sustained ventricular tachycardia associated with sarcoidosis: assessment of the underlying cardiac anatomy and the prospective utility of programmed ventricular stimulation, drug therapy and an implantable anti-tachycardia device. J Am Coll Cardiol 1991;18(4):937-943.

103. Aikawa T, Oyama-Manabe N, Naya M, Ohira H, Sugimoto A, Tsujino I, Obara M, Manabe O, Kudo K, Tsutsui H, Tamaki N. Delayed contrast enhanced computed tomography in patients with known or suspected cardiac sarcoidosis: a feasibility study. Eur Rad 2017;27(10):4054-4063. doi: 10.1007/s00330-017-4824-x.

104. Lee HJ, Im DJ, Youn JC, Chang S, Suh YJ, Hong YJ, Hur J, Choi BW. Assessment of myocardial delayed enhancement with cardiac computed tomography in cardiomyopathies: a prospective comparison with delayed enhancement cardiac magnetic resonance imaging. Int J Cardiovasc Imaging 2017;33(4):577-584. doi: 10.1007/s10554-016-1024-8.

105. Lee HJ, Im DJ, Youn JC, Chang S, Suh YJ, Hong YJ, Hur J, Choi BW. Myocardial extracellular volume fraction with Dual-Energy Equilibrium Contrast-Enhanced Cardiac CT in Non-Ischemic Cardiomyopathy: A prospective comparison with Cardiac MR Imaging. Radiology 2016;280(1):49-57. doi: 10.1148/radiol.2016151289.

106. Smedema JP, Truter R, de Klerk PA, Zaaiman L, White L, Doubell AF. Cardiac sarcoidosis evaluated with gadolinium-enhanced magnetic resonance and contrast-enhanced 64-slice computed tomography. Int J Cardiol 2006;112(2):261-263. doi: 10.1016/j.ijcard.2005.07.059.

107. Muth G, Daniel WG, Achenbach S. Late enhancement on cardiac tomography in a patient with cardiac sarcoidosis. J Cardiovasc Comput Tomogr 2008;2(4):272-273. doi: 10.1016/j.jcct.2008.06.002.

108. Kanao S, Tadamura E, Yumamuro M, Kubo S, Kimura T, Kita T, Togashi K. Demonstration of involvement of sarcoidosis by contrast-enhanced multi-slice computed tomography and delayed-enhanced magnetic resonance imaging. J Comput Assist Tomogr 2005;29:745-748.

109. Sayah DM, Bradfield JS, Moriarty JM, Belperio JA, Lynch JP 3rd. Cardiac involvement in sarcoidosis: evolving concepts in diagnosis and treatment. Sem Resp Crit Care Med 2017;38(4):477-498. doi: 10.1055/s-00371602381.

110. Mañá J. Nuclear imaging. 67 Gallium, 201 Thallium, 18F labeled fluoro-2-deoxy-D-glucose positron emission tomography. Clin Chest Med 1997;18(4):799-811.

111. Mañá J, Gámez C. Molecular imaging in sarcoidosis. Curr Opin Pulm Med 2011;17(5):325-31. doi: 10.1097/MCP.0b013e3283480d36.

112. Aggarwal NR, Snipeliskey D, Young PM, Gersh BJ, Cooper LT, Chareonthaitawee P. Advances in imaging for diagnosis and management of cardiac sarcoidosis. Eur Heart J Cardiovasc Imag 2015;16:949-958. doi:10.1093/ehjci/jev142

113. Le Guludec D, Menad F, Fraggi M, Weinmann MP, Battesti JP, Valeyre D. Myocardial sarcoidosis. Clinical value of technetium-99m sestamibi tomoscintigraphy. Chest 1994;106:1675-82

114. Nakazawa A, Ikeda K, Ito Y, Iwase M, Sao K, Ueda R, Dohi Y. Usefulness of Dual ${ }^{67}$ Gallium/ Tc ${ }^{99}$ Sestamibi SPECT scanning in the diagnosis of cardiac sarcoidosis. Chest 2004;126:1372-1376. 10.1378/chest.126.4.1372.

115. Blankstein R, Osborn M, Naya M, Waller A, Kim CK, Murthy VL, Kazemian P, Kwong RY, Tokuda M, Skali H, Padera R, Hainer J, Stevenson WG, Dorbala S, Di Carli MF. Cardiac positron emission tomography enhances prognostic assessments of patients with suspected cardiac sarcoidosis. J Am Coll Cardiol 2014;63:329-336. doi: 10.1016/j. jacc.2013.09.022

116. Muser D, Santangeli P, Casto SA, Liang JJ, Enriques A, Werner TJ, Nucifora G, Magnani S, Hayashi T, Zado ES, Garcia FC, Callans DJ, Dixit S, Desjardins B, Marchlinski FE, Alavi A. Prognostic role of serial quantitative evaluation of 18F-fluorodeoxyglucose uptake by PET/CT in patients with cardiac sarcoidosis presenting with ventricular tachycardia. Eur J Nucl Med Mol Imaging 2018;45(8):1394-1404. doi: 10.1007/s00259-018-4001-8.

117. Youssef G, Leung E, Mylonas I, Nery P, Williams K, Wisenbeg G, Gulenchyn KY, DeKemp RA, Dasilva J, Birnie D, Wells GA, Beanlands RS. The use of 18F-FDG PET in the diagnosis of cardiac sarcoidosis: a sys- 
tematic review and meta-analysis including the Ontario experience. J Nucl Med 2012;53(2):241-248. doi: 10.2967/jnumed.11.090662.

118. Yamagishi H, Shirai N, Takagi M, Yoshiyama M, Akioka K, Takeuchi K, Yoshikawa J. Identification of cardiac sarcoidosis with 13N-NH3/18F-FDG PET. J Nucl Med 2003;44(7):10s0-1036.

119. Dhar R, Ananthasubramaniam K. Rubium-82 cardiac positron emission tomography imaging: an overview for the general cardiologist. Cardiol Rev 2011;19(5):255-263. doi: 10.1097/CRD.0b013e318224253e.

120. Lapa C, Reiter T, Kircher M, Schirbel A, Werner RA, Pelzer T, Pizarro C, Skowash D, Thomas L, Schlesinger-Irsch U, Thomas D, Bundschuh RA, Bauer WR, Gärtner FC. Somatostatin receptor based PET/CT in patients with the suspicion of cardiac sarcoidosis: an initial comparison to cardiac MRI. Oncotarget 2016;47(7):77807-77814. doi: 10.18632/oncotarget.12799.

121. Reiter T, Werner RA, Bauer WR, Lapa C. Detection of cardiac sarcoidosis by macrophage-directed somatostatin receptor 2-based positron emission tomography/computed tomography. Eur Heart J 2015;36(35):2404. doi: 10.1093/eurheart/ehv278.

122. Pizarro C, Kluenker F, Dabir D, Thomas D, Gaertner FC, Essler M, Grohé C, Nickenig G, Skowasch D. Cardiovascular magnetic resonance imaging and clinical performance of somatostatin positron emission tomography in cardiac sarcoidosis. ESC Heart Failure 2018;5(2):249-261. doi: 10.1002/ehf2.12243.

123. Hotta M, Minamimoto R, Kubota S, Awaya T, Hiroi Y. 11C-4DST PET/CT Imaging of cardiac sarcoidosis: comparison with 18F-FDG and Cardiac MRI. Clin Nucl Med 2014;43(6):458-459. doi: 10.1097/RLU.0000000000002059.

124. White JA, Rajchl M, Butler J, Thompson RT, Prato FS, Wisenberg G. Active cardiac sarcoidosis: first clinical experience of simultaneous positron-emission tomography-magnetic resonance imaging for the diagnosis of cardiac disease. Circulation 2013;127(22):e639-e641. doi: 10.1161/CIRCULATIONAHA.112.001217.

125. Dweck MR, Abgral R, Trivieri MG, Robson PM, Karakatsanis N, Mani V, Palmisano A, Miller MA, Lala A, Chang HL, Sanz J, Contreras J, Narula J, Fuster V, Padilla M, Fayad ZA, Kovacic JC. Hybrid Magnetic Resonance Imaging and Positron Emission Tomography with Fluorodeoxyglucose to diagnose active cardiac sarcoidosis. JACC Cardiovasc Imaging 2018;11(1):94-107. doi: 10.1016/j.cmg.2017.02.021

126. Ohira H, Tsujino I, Ishimaru S, Oyama N, Takei T, Tsukamoto E, Miura M, Sakaue S, Tamaki N, Nishimura M. Myocardial imaging with 18-F-fluorodeoxyglucose positron emission tomography and magnetic resonance imaging in sarcoidosis. Eur J Nucl Med Mol Imaging 2008;35:933-941. doi: 10.1007/s00259-0070650-8.

127. Nekolla SG, Rischpler C, Batrice A, Schwaiger M. Cardiac PET/MRI. Curr Cardiovasc Imaging Rep 2013;6:158-168.

128. Rischpler C, Nekolla SG, Dregely I, Schwaiger M. Hybrid PET/MR imaging of the heart: potential, initial experience, and future prospects. J Nucl Med 2013;54:402-415. doi: 10.2967/jnumed.112.105353.

129. Nensa F, Poeppel TD, Beiderwellen K, Schelhorn J, Mahabadi AA, Erbel R, Heusch P, Nassenstein K, Bockisch A, Forsting M, Schlosser T. Hybrid PET/MR imaging of the heart: feasibility and initial results. Radiology 2013;268(2):366-373. doi: 10.1148/radiol.13130231.

130. Schneider S, Batrice A, Rischpler C, Eiber M, Ibrahim T, Nekolla SG. Utility of multimodal cardiac imaging with PET/MRI in cardiac sarcoidosis: implications for diagnosis, monitoring and treatment. Eur Heart J 2014;35:312. doi: 10.1093/eurheartj/eht335.

131. Smedema JP, van Kroonenburg MJPG, Snoep G, Backes W, Gorgels AP. Cardiac sarcoidosis in a patienst with hypertrophic cardiomyopathy demonstrated with CMR and Single Photon Emission Computed Tomography Dual Isotope Scintigraphy. Circulation 2004;110:e529-e531. doi: 10.1161/01.CIR.0000149749.95902.A4.

132. Smedema JP, Snoep G, van Kroonenburgh MPG, van Geuns RJ, Dassen WR, Gorgels AP, Crijns HJGM. Evaluation of the accuracy of gadolinium-enhanced cardiovascular magnetic resonance in the diagnosis of cardiac sarcoidosis. J Am Coll Cardiol 2005;45:1683-90. doi: 10.1016/j.jacc.2005.01.047.

133. Patel MR, Cawley PJ, Heitner JF, Klem I, Parker MA, Jaroudi WA, Meine TJ, White JB, Elliott MD, Kim HW, Judd RM, Kim RJ. Detection of myocardial damage in patients with sarcoidosis. Circulation 2009;120:1969-77. doi: 10.1161/CIRCLATIONAHA.109.851352.

134. Patel AR, Klein MR, Chandra S, Spencer KT, Decara JM, Lang RM, Burke MC, Garrity ER, Hogarth DK, Archer SL, Sweiss NL, Beshai JF. Myocardial damage in patients with sarcoidosis and preserved left ventricular systolic function: an observational study. Eur J Heart Fail 2011;13:1231-37. doi: 10.1093/eurjhf/hfr099.

135. Hulten E, Agarwal V, Cahill M, Cole G, Vita T, Parrish S, Bittencourt MS, Murthy VL, Kwong R, Di Carli MF, Blankstein R. Presence of late gadolinium enhancement by Cardiac Magnetic Resonance amongst pa- 
tients with suspected cardiac sarcoidosis is associated with adverse cardiovascular prognosis: A systematic review and meta-analysis. Circ Cardiovasc Imaging 2016;9(9):e005001. doi: 10.1161/CIRCIMAGING.116.005001.

136. Coleman GC, Shaw PW, Balfour PC jr, Gonzalez JA, Kramer CM, Patel AR, Salerno M. Prognostic value of myocardial scarring on CMR in patients with cardiac sarcoidosis. JACC Cardiovasc Imaging 2017;10(4):411420. doi: 10.1016/j.jcmg.2016.05.009.

137. JG Scadding, DN Mitchell. Sarcoidosis. $2^{\text {nd }}$ Edition. Springer-Science + Business Media, BV. ISBN 978-0412-21760-9. doi: 10.1007/978-1-4899-2971-6.

138. Gerke AK, Hunninghake G. The immunology of sarcoidosis. Clin Chest Med 2008;29:379-390. doi: 10.1016/j.ccm.2008.03.014.

139. Kuribayashi Y, Ohtani K, Saito T, Ide T. Electron microscopy gain a glimpse into the pathogenesis of cardiac sarcoidosis. Eur Heart J Cardiovasc Imaging 2017;18(8):944. doi: 10.1093/ehjci/jex152.

140. Iwai K, Takemura T, Kitaichi M, Kawabata Y, Matsui Y. Pathological studies on sarcoidosis autopsy. II Early change, mode of progression and death pattern. Acta Pathol Japonica 1993:43:377-385.

141. Chen ES, Moller DR. Etiology of sarcoidosis. Clin Chest Med 2008;29:365-377.

142. Epelman S, Liu PP, Mann PL. Role of innate and adaptive immunity in cardiac injury and repair. Nat Rev Immunol 2015;15(2):117-129. doi:10.1038/nri3800.

143. Munshi VK. Resident macrophages: Near and dear to your heart. Editorial. Cell 2017;169:376-377. doi: 10.1016//j.cell.2017.04.002.

144. Hulsmans M, Clauss S, Xiao L, Aguirre AD, King KR, Hanley A, Hucker WJ, Wülfers AMN, Seemann G, Courties G, Iwamoto Y, Sun Y, Savol AJ, Sager HB, Lavine KJ, Fishbein AG, Capen DE, Da Silva N, Miquerol L, Wakimoto H, Seidman SE, Seidman JG, Sadreyev RI, Naxerova K, Mitchell RN, Brown D, Libby P, Weissleder R, Swirski FK, Kohl P, Vinegoni C, Milan DJ, Ellinor PT, Nahrendorff M. Macrophages facilitate electrical conduction in the heart. Cell 2017;169:510-522.http://dx.doi.org/10.1016/j.cell.2017.03.050.

145. Virmani R, Bures JC, Roberts WC. Cardiac sarcoidosis. A major cause of sudden death in young individuals. Chest 1980;77:423-428.

146. Hamzeh N, Steckman DA, Sauer WH, Judson MA. Pathophysiology and clinical management of cardiac sarcoidosis. Nature Rev Cardiol 2015;12:278-288. doi: 10.1038/nrcardio.2015.22.

147. Okura Y, Dec GW, Hare JM, Kodama M, Berry GJ, Tazelaar HD, Bailey KR, Cooper LT. A clinical and histopathological correlation of cardiac sarcoidosis and idiopathic giant cell myocarditis. J Am Coll Cardiol 2003;41(2): 322-329.

148. Blauwet LA, Cooper LT. Ideopathic giant cell myocarditis and cardiac sarcoidosis. Heart Fail Rev 2013;1896):733-746. doi: 10.1007/s10741-012-9358-3.

149. Silber MD, Gotlieb AI, Schoen FJ. Cardiovascular pathology. Third edition, 2001. Churchill Livingstone.ISBN 0-443-06535-7 Chapter 9. Myocarditis. Chapter 16. Cardiovascular effects of systemic diseases and conditions.

150. Cooper LT, Ed. Myocarditis from bench to bedside. Humana Press 2002. ISBN 978-1-59259-319-4.

151. Ferrans VJ, Rodriguez ER, McAllister HA Jr. Granulomatous inflammation of the heart. Heart Vessels Suppl 1985;1:262-270.

152. Prasse A. The diagnosis, differential diagnosis and treatment of sarcoidosis. Dtsch Artzebl Int 2016;113:565574. doi: 10.3238/arztebl.2016.0565.

153. Ferrans VJ, Hibbs RG, Black WC, Walsh JJ, Burch GE. Myocardial degeneration in cardiac sarcoidosis. Histochemical and electron microscopic studies.Am Heart J 1965;2:159-172.

154. Mitchell DG. MRI Principles. WB Saunders Company. 1999 ISBN 0-7216-6759-7

155. Bushong S. Magnetic Resonance Imaging. Third Edition. Mosby 2003. ISBN 0-323-01485-2

156. Hurst's The Heart. Fourteenth Edition. Chapter 16: Magnetic Resonance Imaging of the Heart. McGraw Hill 2017. ISBN 0-0718-4311-6.

157. Kramer CM, Barkhausen J, Flamm SD, Kim RJ, Nagel E. Standardized cardiovascular magnetic resonance (CMR) protocols 2013 update. J Cardiovasc Magn Res 2013;15:91. doi: 10.1186/1532-429X-15-91.

158. Schulz-Menger J, Bluemke D, Bremerich J, Flamm Sd, Fogel MA, Friedrich MG, Kim RJ, von Knobelsdorff-Brenkenhoff F, Kramer CM, Pennell DJ, Plein S, Nagel E. Standard image interpretation and post processing in cardiovascular magnetic resonance: society for cardiovascular magnetic resonance (SCMR) board of trustees task force on standard post processing. J Cardiovasc Magn Reson 2013;15:35. doi: 10.1186/1532-429X-15-35. 
159. Vignaux O, Dhôte R, Duboc D, Blanche P, Devaux JY, Weber S, Legmann P. Detection of myocardial involvement in patients with sarcoidosis applying T2-weighted, contrast-enhanced and cine magnetic resonance imaging: initial results of a prospective study. J Comput Assist Tomograph 2002;26:762-767.

160. Vignaux O, Dhôte R, Duboc D, Blanche P, Dusser D, Weber S, Legmann P. Clinical significance of myocardial magnetic resonance abnormalities in patients with sarcoidosis. A 1-year follow-up study. Chest 2002;122:1895-1901.

161. Vignaux O. Cardiac sarcoidosis: spectrum of MRI features. AJR Am J Roengenol 2005;184:249-54. doi: 10.2214/ajr.184.1.01840249.

162. Shimada T, Shimada K, Sakane T, Ochiai K, Tsukihashi H, Fukiu M, Inoue S, Katoh H, Murakami Y, Ishibashi Y, Maruyama R. Diagnosis of cardiac sarcoidosis and evaluation of the effects of steroid therapy by gadolinium-DTPA-enhanced magnetic resonance imaging. Am J Med 2001;110:520-27.

163. Parsai C, O’Hanlon R, Prasad SK, Mohiaddin RH. Diagnostic and prognostic value of cardiovascular magnetic resonance in non-ischemic cardiomyopathies. J Cardiovasc Magn Reson 2012;14(1):54. doi:10.1186/1532-429X-14-54.

164. Bogaert J, Taylor AM. Clinical Cardiac MRI. Bogaert J, Dymarkowski S, Taylor AM, Muthurangu V. Eds. Chapter 7. Pages 275-352. Heart muscle diseases. Springer-Verlag Berlin Heidelberg 2012. doi: 10.1007/174_2011_360

165. Stauder NI, Bader B, Fenchel M, Kramer U, Kuhlkamp V, Miller S. Images in cardiovascular medicine. Follow-up of cardiac sarcoidosis by magnetic resonance imaging. Circulation 2005;111(11):e158-60. doi: 10.1161/01.CIR.0000158436.18971.A1.

166. Stanton KM, Ganigara M, Corte P, Celermajer DS, McGuire MA, Torzillo PJ, Corte TJ, Puranik R. The utility of cardiac magnetic resonance imaging in the diagnosis of cardiac sarcoidosis. Heart Lung Circ 2017;26(11):1191-1199. doi: 10.1016/j.hlc.2017.02.021.

167. Orii M, Hirata K, Tanimoto T, Ota S, Shiono Y, Yamano T, Matsuo Y, Ino Y, Yamaguchi T, Kubo T, Tanaka A, Akasaka T. Comparison of cardiac MRI and 18F-FDG positron emission tomography manifestations and regional response to corticosteroid therapy in newly diagnosed cardiac sarcoidosis with complete heart block. Heart Rhythm 2015;12(12):2477-2485. doi: 10.1016/j.hrthm.2015.06.031.

168. Mahrhold H, Wagner A, Judd RM, Sechtem U, Kim RJ. Delayed enhancement cardiovascular magnetic resonance assessment of non-ischemic cardiomyopathies. Eur Heart J 2005;26:1461-1474. doi:10.1093/eurheartj/ehi258

169. Bogabathina H, Olson P, Rathi VK, Biederman RWW. Case Report: Cardiac Sarcoidosis or Giant Cell Myocarditis? On treatment improvement of fulminanant myocarditis as demonstrated by Cardiovascular Magnetic Resonance Imaging. Case Rep Cardiol 2012;2012:647041. doi: 10.1155/2012/647041.

170. Slater GM, Rodriguez EM, Lima JAC, Bluemke DA. A unique presentation of cardiac sarcoidosis. Am J Roentgenol 2003;180:1738-1739. doi: 10.2214/ajr.180.6.1801738.

171. Greulich S, Kitterer D, Latus J, Aguor E, Steubing H, Kaesemann P, Patrascu A, Greiser A, Groeninger S, Mayr A, Braun N, Alscher D, Sechtem U, Mahrholdt H. Comprehensive cardiovascular magnetic resonance assessment in patients with sarcoidosis and preserved left ventricular ejection fraction. Circ Cardiovasc Imaging 2016;9:e005022. doi: 10.1161/CIRCIMAGING.116.005022.

172. Crouser ED, Ono C, Tran T, He X, Raman SV. Improved detection of cardiac sarcoidosis using magnetic resonance with myocardial T2 mapping. Am J Resp Crit Care Med 2014;189:109-112. doi: 10.1164/rccm.201309-1668LE.

173. Puntmann VO, Isted A, Hinojar R, Foote L, Carr-White G, Nagel E. T1 and T2 mapping in recognition of early cardiac involvement in sarcoidosis. Radiology 2017;285(1):63-72. doi: 10.1148/radiol.2017162732.

174. Kadkhodayan A, Chareonthaitawee P, Raman SV, Cooper LT. Imaging of inflammation in unexplained cardiomyopathy. JACC Cardiovascular Imaging 2016;9(5):603-617. doi: 10.1016/j.jcmg.2016.01.010.

175. Schulz-Menger J, Strohm O, Dietz R, Friedrich MG. Visualization of cardiac involvement in patients with systemic sarcoidosis applying contrast-enhanced magnetic resonance imaging. MAGMA 2000;11(1-2):82-83.

176. Greulich S, Deluigi CC, Gloekler S, Wahl A, Zürn C, Kramer U, Nothnagel D, Bültel H, Schumm J, Grün S, Ong P, Wagner A, Schneider S, Nassenstein K, Gawaz M, Sechtem U, Bruder O, Mahrholdt H. CMR Imaging predicts death and other adverse events in suspected cardiac sarcoidosis. J Am Coll Cardiol Img 2013;6:501-11. doi: 10.1016/j.jcmg.2012.10.021.

177. Jiji RS, Kramer CM. Cardiovascular magnetic resonance: Applications in daily practice. Cardiol Rev 2011;19(5):246-254. doi: 10.1097/CRD.0b013e31821f4d6a. 
178. Moraes LG, Higgins CB, Ordovas KG. Delayed enhancement magnetic resonance imaging in non-ischemic myocardiac disease. J Thor Imaging 2013;28:84-95. doi: 10.1097/RTI.0b013e3182828f89.

179. Dabir D, Meyer D, Kuetting D, Luetkens J, Homsi R, Pizarro C, Nadal J, Thomas D. Diagnostic value of cardiac magnetic strain analysis for detection of cardiac sarcoidosis. Fortschr Röntgenstr 2018;190:712-721. doi: 10.1055/a-0598-5099.

180. Fujimoto R, Asano T, Maezawa H, Shimojima H, Tsujiuchi M, Hori Y, Ebato M, Suzuki H. Cardiac sarcoidosis mimicking septal tumor with intermittent complete atrioventricular block. Int Heart J 2018 Nov 28;59(6):1473-1479. doi: 10.1536/ihj.17-713.

181. Riedy K, Fisher MR, Belic N, Koenigsberg DI.MR Imaging of myocardial sarcoidosis. Am J Roentgenol 1988;151:915-916.

182. Takahashi Y, Izumi C, Miyake M, Nakajima S, Nishimura S, Kuroda M, Yoshikawa Y, Amano M, Hayama Y, Imamura S, Onishi N, Tamaki Y, Enomoto S, Tamura T, Kondo H, Kaitani K, Nakagawa Y. Detecting cardiac sarcoidosis with a right atrial mass using transthoracic echocardiography. Intern Med 2016;55(4):359363. doi: 10.2169/internalmedicine.55.5423.

183. Schulz-Menger J, Wassmuth R, Abdel-Aty H, Siegel I, Franke A, Dietz R, Friedrich MG. Patterns of myocardial inflammation and scarring in sarcoidosis as assessed by cardiovascular magnetic resonance. Heart 2006;92:399-400. doi: 10.1136/hrt.2004.058016.

184. Sato T, Kanzaki H, Ishida Y, Amaki M, Ohara T, Hasegawa T, Hashimura K, Nakatani S, Yamada N, Ikeda Y, Ueda-Ishibashi H, Kitakaze M. Second left ventricular aneurysm newly developed in a patients with untreated cardiac sarcoidosis. Circ J 2010;74(11): 2477-2478. doi:

185. Saitou T, Nagai T, Kanda S, Yoshioka K, Ikari Y. A 30-day drastic thinning of the basal interventricular septum in isolated cardiac sarcoidosis masquerading as a hypertrophic cardiomyopathy. Eur Heart J Cardiovasc Imaging 2018 Nov 6. doi: 10.1093/ehjci/jey170 [Epub ahead of print]

186. Ni Y. Clinical Cardiac MRI. Bogaert J, Dymarkowski S, Taylor AM, Muthurangu V. Eds. Chapter 2. Pages 32-51. MR contrast agents for cardiac imaging. Springer-Verlag Berlin Heidelberg 2012. doi: 10.1007/174_2011_360.

187. Vöhringer M, Mahrhold H, Yilmaz A, Sechtem U. Significance of late gadolinium enhancement in Cardiovascular Magnetic Resonance Imaging. Herz 2007:32:129-137. doi: 10.1007/s00057-007-2972-5.

188. Hashimura H, Kimura F, Ishibashi-Ueda H, Morita Y, Higashi M, Nakano S, Igushi A, Uotani K, Sugimura K, Naito H. Radiologic-Pathologic correlation of primary and secondary cardiomyopathies: MR Imaging and histopathological findings in hearts from autopsy and transplantation. Radiographics 2017;37:0000-0000. doi: 10.1148/rg.2017160082

189. Cheong BY, Muthupillai R, Nemeth M, Lambert B, Dees D, Huber S, Castriotta R, Flamm SD. The utility of delayed-enhancement magnetic resonance imaging for identifying non-ischemic myocardial fibrosis in asymptomatic patients with biopsy-proven systemic sarcoidosis. Sarcoidosis Vasc Diffuse Lung Dis 2009;26:39-46. doi:

190. Rudolph A, von Knobelsdorff-Brenkenhoff F, Wassmuth R, Prothmann M, Utz W, Schulz-Menger J. Gadopentetate diglumine and gadobenate dimeglumine for enhanced cardiovascular magnetic resonance imaging. J Magn Res Imaging 2014;39:1153-1160. doi:

191. Hunt $\mathrm{CH}$, Hartman RP, Hesley GK. Frequency and severity of adverse effects of iodinated and gadolinium contrast materials: retrospective review of 456930 doses. AJR Am J Roentgenol 2009;193:1124-1127. doi:

192. Jung JW, Kang HR, Kim HM, Lee W, Min KU, Han MH, Cho SH. Immediate hypersensitivity reaction to gadolinium-based MR contrast media. Radiology 2012;264:414-422. doi: 10.1148/radiol.12112025.

193. Bruder O, Schneider, Nothnagel D, Pilz G, Lombardi M, Sinha A, Wagner A, Dill T, Frank H, van Rossum AC, Schwitter J, Nagel E, Senges J, Sabin G, Sechtem U, Mahrhold A. Acute adverse reaction to gadoliniumbased contrast agents in CMR: multi-centre experience in 17767 patients from the Euro CMR Registry. JACC Cardiovasc Imaging 2011;4:1171-1176. doi: 10.1016/j.jcmg.2011.06.019.

194. FDA drug safety communication: new warnings for using gadolinium-based contrast agents in patients with kidney dysfunction. 12/10 Update. fda.gov

195. Simonetti OP, Kim RJ, Fieno DS, Hillenbrand HB, Wu E, Bundy JM, Finn JP, Judd RM. An improved imaging technique for the visualization of myocardial infarction. Radiology 2001;218(1):215-223. doi: 10.1148/radiology.218.1.r01ja50215.

196. Kim RJ, Shah DJ, Judd RM. How we perform delayed enhancement imaging. J Cardiovasc Magn Res 2003;5(3):505-514. 
197. Smedema JP, Snoep G, van Kroonenburgh MPG, van Geuns RJ, Cheriex EC, Gorgels APM, Crijns HJGM. The additional value of gadolinium-enhanced MRI to standard assessment for cardiac involvement in patients with pulmonary sarcoidosis. Chest 2005:128:1629-1637. doi: 10.1378/chest.128.3.1629.

198. Flett AS, Hasleton J, Cook C, Hausenloy D, Quarta G, Ariti C, Muthurangu V, Moon JC. Evaluation of techniques for the quantification of myocardial scar of differing etiology using cardiac magnetic resonance. J Am Coll Cardiol 2011;4:150-156. doi: 10.1016/j.jcmg.2010.11.015.

199. White SK, Flett AS, Moon JC. Automated scar quantification by CMR: a step in the right direction. J Thorac Dis 2013;5:381-382. doi: 10.3978/j.issn.2072-1439.2013.07.22.

200. Kwong RY, Farzaneh-Far A. Measuring myocardial scar by CMR. J Am Coll Cardiol Img 2011;4(2):157-160. doi: 10.1016/j.jcmg.2010.12.004.

201. Agoston-Coldea L, Kouaho S, Sacre K, et al. High mass ( $>18 \mathrm{~g}$ ) of late gadolinium enhancement on CMR imaging is associated with major cardiac events on long-term outcome in patients with biopsy proven extracardiac sarcoidosis. Int J Cardiol 2016;222:950-6. doi: 10.1016/j.ijcard.2016.07.233.

202. Spiewak M, Malek LA, Misko J, Chojnowska L, Milsz B, Klopotowski M, Petryka J, Dabrowski M, Kepka C, Ruzyllo W. Comparison of different quantification methods of late gadolinium enhancement in patients with hypertrophic cardiomyopathy. Eur J Radiol 2010;74:e149-e154. doi: 10.1016/j.ejrad.2009.05.035.

203. Hundley WG, Bluemke D, Bogaert JG, Friedrich MG, Higgins CB, Lawson MA, McConnell MV, Raman SV, van Rossum AC, Flamm S, Kramer CM, Nagel E, Neubauer S. Society for Cardiovascular Magnetic Resonance guidelines for reporting cardiovascular magnetic resonance examinations. Journal of Cardiovascular Magnetic Resonance 2009;11:5 doi:10.1186/1532-429X-11-5

204. Crawford T, Mueller G, Sarsam S, Prasitdumrong H, Chaiyen N, Gu X, Schuller J, Kron J, Nour KA, Cheng A, Ji SY, Feinstein S, Gupta S, Ilg K, Sinno M, Abu-Hashish S, Al Mallah M, Sauer WH, Ellenbogen K, Morady F, Bogun F. Magnetic Resonance Imaging for identifying patients with cardiac sarcoidosis and preserved or mildly reduced left ventricular function at risk of ventricular arrhythmias. Circ Arrhythm Electrophys 2014;7(6):1109-1115. doi: 10.1161/CIRCEP.113.000156.

205. Mongeon FP, Jerosch-Herold M, Coelho-Filho OR, Blankstein R, Falk RH, Kwong RY. Quantification of extracellular matrix expansion by CMR in infiltrative heart disease. JACC Cardiovasc Imaging 2012;5(9):897907. doi: 10.1016/j.jcmg.2012.04.006.

206. Ugander M, Oki AJ, Hsu LY, Kellman P, Greiser A, Aletras AH, Sibley CT, Chen My, Bandettini WP, Arai AE. Extracellular volume imaging by magnetic resonance imaging provides insights into overt and subclinical myocardial pathology. Eur Heart J 2012;33(10):1268-1278. doi: 10.1093/eurheartj/ehr481.

207. Kammerlander AA, Marzluf BA, Zotter-Tufaro C, Aschauer S, Duca F, Bachmann A, Knechtelsdorfer K, Wiesinger M, Pfaffenberger S, Greiser A, Lang IM, Bonderman D, Mascherbauer J. T1mapping by CMR Imaging: from histological validation to clinical implication. J Am Coll Cardiol Img 2016;9:14-23. doi: 10.1016/j.jcmg.2015.11.002.

208. Liu A, Wijesurendra RS, Francis J, Robson MD, Neubaeur S, Piechnik SK, Ferreira VM. Adenosine stress and rest T1 mapping can differentiate between ischemic infarcted remote and normal myocardium without the need for gadolinium contrast agents. J Am Coll Cardiol Imaging 2016;9:27-36. doi: 10.1016/j.jcmg.2015.08.018.

209. Salerno M, Sharif B, Arheden H, Kumar A, Axel L, Li D, Neubauer S. Recent advances in cardiovascular magnetic resonance. Techniques and applications. Circ Cardiovasc Imaging 2017;10:e003951. doi: 10.1161/ circimaging.116.003951

210. Haaf P, Garg P, Messroghli DR, Broadbent DA, Greenwood JP, Plein S. Cardiac T1 Mapping and Extracellular Volume (ECV) in clinical practice: a comprehensive review. J Cardiovasc Magn Res 2016;18:89. doi: 10.1186/s12968-016-0308-4.

211. Puntmann VO, Carr-White G, Jabbour A, Yu CY, Gebker R, Kelle S, Hinojar R, Doltra A, Varma M, Child N, Rogers T, Suna G, Ucar EA, Goodman B, Khan S, Dabir D, Herrmann E, Zeiher AM, Nagel E. T1mapping and outcome in nonischemic cardiomyopathy. J Am Coll Cardiol Img 2016;9:40-50. doi: 10.1016/j.jcmg.2015.12.001.

212. Messroghli DR, Moon JC, Ferreira V, Grosse-Wortmann L, He T, Kellman P, Mascherbauer J, Nezafat R, Salerno M, Schelbert EB, Taylor AJ, Thompson R, Ugander M, van Heesweek RB, Friedrich MG. Clinical recommendations for cardiovascular magnetic resonance mapping of T1, T2, T2* and extracellular volume: A consensus statement by the Society for Cardiovascular Magnetic Resonance (SCMR) endorsed by the European Association for Cardiovascular Imaging (EACVI). J Cardiovascular Magn Res 2017;19:75-99. doi: 10.1186/s12968-017-0389-8. 
213. Isted A, Grigoratos C, Bratis K, Carr-White G, Nagel E, Puntmann VO. Correspondence. Native T1 in deciphering the reversible myocardial inflammation in cardiac sarcoidosis with anti-inflammatory treatment. Int J Cardiol 2016;203:459-462. doi: 10.1016/j.ijcard.2015.10.199.

214. Treibel TA, Moon JC. Synthetic extracellular volume fraction - state of play. Wiener Klin Wochenschr 2018;130(5-6):165-167. doi: 10.1007/s00508-01701287-7

215. Treibel TA, Fontana M, Maestrini V, Castelletti S, Rosmini S, Simpson J, Nasis A, Bhuva AN, Bulluck H, Abdel-Gadir A, White SK, Manisty C, Spottiswoode BS, Wong TC, Piechnik SK, Kellman P, Robson MD, Schelbert EB, Moon JC. Automatic measurement of the myocardial interstitium: synthetic extracellular volume quantification without hematocrit sampling. JACC Cardiovasc Imaging 2016;9:54-63. doi: 10.1016/j.jjcmg.2015.11.008

216. Kammerlander AA, Duca F, Binder C, Aschauer S, Zotter-Tufaro C, Koschutnik M, Marzluf BA, Bonderman D, Macherbauer D. Extracellular volume quantification by cardiac magnetic resonance imaging without hematocrit sampling. Wiener Klin Wochenschr 2018;130(5-6):190-196. doi: 10.1007/s00508-017-1267-y

217. Fent GJ, Garg P, Foley JRJ, Swoboda PP, Dobson LE, Erhayiem B,Treibel TA, Moon JC, Greenwood JP, Plein S. Synthetic myocardial extracellular volume fraction. JACC Cardiovasc Imaging 2017;10(11):14021404. doi: 10.1016/j.jcmg.2016.12.007.

218. Nyktari E, Vassiliou VS, Arzanauskaite M, Gatehouse P, Greiser A, Wechalekar A, Gilbertson J, Pierce I, Sharma R, Mohiaddin R. Challenging Occam's Razor: An unusual combination of sarcoidosis and amyloidosis. The value of cardiac magnetic resonance imaging in infiltrative cardiomyopathies. Can J Cardiol 2017;33(10):1335.e9-1335.e11. doi:10.1016/j.cjca.2017.07.004

219. Cameron D, Vassiliou VS, Higgins DM, Gatehouse PD. Towards accurate and precise T1 and extracellular volume mapping in the myocardium: a guide to current pitfalls and their solutions. MAGMA 2018;31(1):143-163. doi 10.1007/s10334-017-0631-2.

220. Mascherbauer J. Myocardial inflammation: An important pitfall during CMR T1mapping for the quantification of diffuse fibrosis in heart failure. JACC Cardiovasc Imag 2018;11(1):46-47. doi:10.1016/j.jcmg.2017.03.003.

221. Saeed M, Liu H, Liang CH, Wilson MW. Magnetic resonance imaging for characterizing myocardial diseases. Int J Cardiovasc Imaging 2017;33(9):1395-1414. doi: 10.1007/s10554-017-1127.

222. Miyazaki S, Funabashi N, Nagai T, Uehara M, Kataoka A, Takaoka H, Ueda M, Komuro I. Cardiac sarcoidosis complicated with atrioventricular block and wall thinning, edema and fibrosis in left ventricle: confirmed recovery to normal sinus rhythm and visualization of edema improvement by administration of prednisolone. Int J Cardiol 2011;150(1):e4-10. doi:

223. Mirakhur A, Anca N, Mikami Y, Merchant N. T2-weighted imaging of the heart - a pictoral review. Eur J Radiol 2013;82(10):1755-1762. doi: 10.1016/j.ejrad.2013.06.005.

224. Lota AS, Gatehouse PD, Mohiaddin RH. T2 mapping and T2* imaging in heart failure. Heart Fail Rev 2017;22:431-440. doi: 10.1007/s10741-017-9616-5.

225. Mavrogeni SI, Kitas GD, Dimitroulas T, Sfikakis PP, Seo P, Gabriel S, Patel AR, Gargani L, Bombardieri S, Matucci-Cerinic M, Lombardi M, Pepe A, Aletras AH, Kolovou G, Miszalski T, van Riel P, Semb AG, Gonzalez-Gay MA, Dessein P, Karpouzas G, Puntmann V, Nagel E, Bratis K, Karabela G, Stravropoulos E, Katsifis G, Koutsogeorgopoulou L, van Rossum AC, Rademakers F, Pohost G, Lima JAC. CMR in rheumatology: Current status and recommendations for use. Int J Cardiol 2016;135-148. doi: 10.1016/j.ijcard.2016.04.158

226. Wu KC. Sudden cardiac death substrate imaged by Magnetic Resonance Imaging. From investigational tool to clinical applications. Circ Cardiovasc Imaging 2017;10:e005461. doi: 10.1161/circimaging.116.005461

227. Crouser ED, Ruden E, Julian MW, Raman SV. Resolution of abnormal cardiac MRI T2 signal following immune suppression for cardiac sarcoidosis. J Investig Med 2016;64(6):1148-50. doi: 10.1136/jim-2016000144.

228. Ferreira VM, Piechnik SK. Seing beyond the obvious. Subclinical cardiac sarcoidosis revealed by cardiovascular magnetic resonance mapping. Circ Cardiovasc Imaging 2016;9:e005592.

229. Hamlin SA, Henry TS, Little BP, Lerakis S, Stillman AE. Mapping the future of cardiac MR imaging: casebased review of T1 and T2 mapping techniques. Radiographics 2014;34(6):1594-1561. doi: 10.1148/rg.346140030.

230. Muser D, Santangeli P, Patahk RK, Castro SA, Liang JJ, Magnani S, Hayashi T, Garcia FC, Hutchinson MD, Supple GE, Frankel DS, Riley MP, Lin D, Schaller RD, Desjardin B, Dixit S, Callans DJ, Zado ES, March- 
linski FE. Long-term outcomes of catheter ablation of ventricular tachycardia in patients with cardiac sarcoidosis. Circ Arrhythm Electr 2016;9(8):e004333. doi: 10.1161/CIRCEP.116.004333.

231. Sadek MM, Yung D, Birnie DH, Beanlands RS, Nery PB. Corticosteroid therapy for cardiac sarcoidosis: a systemic review. Can J Cardiol 2013;29:1034-1041. doi: 10.1016/j.cjca.2013.02.004

232. Orii M, Hirata K, Tanimoto T, Ota S, Shiono Y, Yamano T, Matsuo Y, Ino Y, Yamaguchi T, Kubo T, Tanaka A, Akasaka T. Comparison of cardiac MRI and 18F-FDG positron emission tomography manifestations and regional response to corticosteroid therapy in newly diagnosed cardiac sarcoidosis with complete heart block. Heart Rhythm 2015;12(12):2477-2485. doi: 10.1016/j.hrthm.5015.06.032.

233. Orii M, Imanishi T, Akasaka T. Assessment of cardiac sarcoidosis with advanced imaging modalities. BioMed Res Int 2014:897956. doi: 10.1155/2014/897956.

234. Zandieh S, Bernt R, Mirzaei S, Haller J, Hergan K. Image fusion between 18F-FDG PET and MRI in cardiac sarcoidosis: a case series. J Nucl Cardiol 2018;25(4):1128-1134. doi: 10.1007/s12350-016-0653-6.

235. Nazir MS, Ismail TF, Reyes E, Chiribiri A, Kaufmann PA, Plein S. Hydrid positron emission tomography magnetic resonance of the heart: current state of the art and future applications. Eur Heart J Cardiovasc Imag 2018;19:962-974. doi:10.1093/ehjcvi/jey090

236. Bietenbeck M, Florian A, Faber C, Sechtem U, Yilmaz A. Remote magnetic targeting of iron oxide nanoparticles for cardiovascular diagnosis and therapeutic drug delivery: were are we now? Int J NanoMed 2016;1:3191-3203. doi: 10.2147/IJN.S110542.

237. Stirrat CG, Alam SR, MacGillivray TJ, Gray CD, Dweck MR, Raftis J, Jenkins WSA, Wallace WA, Pesotto R, Lim KHH, Mirsadraee S, Hendriksen PA, Semple SIK, Newby DE. Ferumoxytol-enhanced magnetic resonance imaging assessing inflammation after myocardial infarction. Heart 2017;0:1-8. doi:10.1136/heartjnl-2016-311018

238. Moon H, Park HE, Kang J, Lee H, Cheong C, Lim YT, Ihm SH, Seug KB, Jaffer FA, Narula J, Chang K, Hong KS. Noninvasive assessment of myocardial inflammation by Cardiovascular Magnetic Resonance in a rat model of experimental autoimmune myocarditis. Circulation 2012;125:2603-2612. doi: 10.1161/CIRCULATIONAHA.111.075283.

239. Jacoby C, Borg N, Heusch P, Sauter M, Bönner F, Kandolf R, Klingel K, Schrader J, Flögel U. Visualization of immune cell infiltration in experimental viral myocarditis by ${ }^{19} \mathrm{~F}$ MRI in vivo. Magn Reson Mater Phy 2014;27:101-106. doi: 10.1007/s10334-013-0391-6.

240. Van Heeswijk RB, de Blois J, Kania G, Gonzales C, Blyszczuk P, Stuber M, Eriksson U, Schwitter J. Selective in vivo visualization of immune-cell infiltration in a mouse model of auto-immune myocarditis by fluorine-19 cardiac magnetic resonance. Circ Cardiovasc Imaging 2013;6:277-284. doi: 10.1161/CIRCIMAGING.112.000125.

241. Schuller JL, Zipse M, Crawford T, Bogun F, Beshai J, Patel AR, Sweiss NJ, Nguyen DT, Aleong RG, Varosy PD, Weinberger HD, Sauer WH. Implantable cardioverter defibrillator therapy in patients with cardiac sarcoidosis. J Cardiovasc Electrophysiol 2012;23(9):925-929. doi: 10.1111/j.1540-8167.2012.02350.

242. Strom JB, Whelan JB, Shen C, Sheng SQ, Moretele KJ, Kramer DB. Safety and utility of magnetic resonance imaging in patients with cardiac implantable electronic devices. Heart Rhythm 2017;14(8):1138-1144. doi: 10.1016/j.hrthm.2017.03.039.

243. Shulman RM, Hunt B. Cardiac implanted electronic devices and MRI safety in 2018-the state of play. Eur Radiol 2018;28(10):4062-4065. doi: 10.1007/s00330-018-5396-0.

244. Ferreira AM, Costa F, Tralhao A, Marques H, Cardim N, Adragao P. MRI-conditional pacemakers: current perspectives. Medical Devices: Evidence and Research 2014;7:115-124.

245. Dupuis JM, Victor J, Furber A, Pézard P, Lejeune LL, Tadei A. Value of magnetic resonance imaging in cardiac sarcoidosis. Apropos of a case. Arch Mal Coeur Vaiss 1994;87(1):105-10.

246. Chandra M, Silverman ME, Oshinski J, Pettigrew R. Diagnosis of cardiac sarcoidosis aided by MRI. Chest 1996;110:562-565.

247. Yoshida S, Ishibashi-Ueda H, Yamada N, Kanzaki H, Hasegawa T, Takahama H, Amaki M, Asakura M, Kitakaze M. Direct comparison of the diagnostic capability of cardiac magnetic resonance and endomyocardial biopsy in patients with heart failure. Eur J Heart Fail 2013;15:166-175. doi: 10.1093/eurjhf/hfs206.

248. Ichinose A, Otani H, Oikawa M, Takase K, Saito H, Shimokawa H, Takahashi S. MRI of cardiac sarcoidosis: basal and subepicardial localization of myocardial lesions and their effect on left ventricular function. AJR Am J Roentgenol 2008;191:862-869. doi: 10.2214/AJR.07.3089.

249. Matsumori A, Hara M, Nagai S, Izumi T, Ohashi N, Ono K, Sasayama S. Hypertrophic cardiomyopathy as a manifestation of cardiac sarcoidosis. Jpn Circ J 2000;64(9):679-683. 
250. Japanese Ministery Health Welfare. Diagnostic standard and guidelines for sarcoidosis. Jpn J Sarcoidosis Granulomatous Disord (Jpn) 2007;27:89-102.

251. Soejima K, Yada H. The work-up and management of patients with apparent or subclinical cardiac sarcoidosis: with emphasis on the associated heart rhythm abnormalities. J Cardiovasc Electrophysiol 2009;20:578583. doi: 10.1111/j.1540-8167.2008.01417.

252. Costabel U, Skowasch D, Pabst S, Störk S, Schöpe C, Allewelt M, Worth H, Müller-Quernheim J, Grohé C. Diagnostik and Therapie der kardialen Sarkoidose. Pneumatologie 2014;68:124-132. doi: 10.1055/s-00331359197.

253. Judson MA, Costabel U, Drent M, Wells A, Maier L, Koth L, Shigemitsu H, Culver DA, Gelfand J, Valeyre D, Sweiss N, Crouser E, Morgenthau AS, Lower EE, Azuma A, Ishihara M, Morimoto S, Yamaguchi T, Shijubo N, Grutters JC, Rosenbach M, Li HP, Rottoli P, Inoue Y, Prasse A, Baughman RP. The WASOG Sarcoidosis Organ Assessment Instrument: An update of a previous clinical tool. Sarcoidosis Vasculitis and Diffuse Lung Diseases 2014;31:19-27. doi:

254. Terasaki F, Yoshinaga K. New guidelines for diagnosis of cardiac sarcoidosis in Japan. Ann Nucl Cardiol 2017;3:42-45. doi: 10.17996/anc.17-00042.

255. Matoh F, Satoh H, Shiraki K, Odagiri K, Saitoh T, Urushida T, Katoh H, Takehara Y, Sakahara H, Hayashi $\mathrm{H}$. The usefulness of delayed enhanced magnetic imaging for diagnosis and evaluation of cardiac function in patients with cardiac sarcoidosis. J Cardiol 2008;51:179-188. doi: 10.1016/j.jjcc.2008.03.002.

256. Watanabe E, Kimura F, Nakajima T, Hiroe M, Kasai Y, Nagata M, Kawana M, Hagiwara N. Late gadolinium enhancement in cardiac sarcoidosis: characteristic magnetic resonance findings and relationship with left ventricular function. J Thorac Imaging 2013:28:60-66. doi: 10.1097/RTI.0b013e3182761830.

257. Yatsynovitch Y, Valencia D, Petrov M, Linares JD, Rahman MM, Dittoe N. Curr Treatm Options Cardiovasc Med 2018;20(9):74. doi: 10.1007/s11936-018-0670-7.

258. Bravo PE, Singh A, Di carli MF, Blankstein R. Review article. Advanced cardiovascular imaging for the evaluation of cardiac sarcoidosis. J Nucl Cardiol 2018 Nov 2. doi: 10.1007/s12350-018-01488-9. [Epub ahead of print]

259. Kandolin R, Lehtonen J, Graner M, Schildt J, Salmenkivi K, Kivistö SM, Kupari M. Diagnosing isolated cardiac sarcoidosis. J Intern Med 2011;270:461-468. doi:

260. Yazaki Y. The diagnostic dilemma of isolated cardiac sarcoidosis. Internal Medicine 2013;52(1):1-2.

261. Isobe M, Tezuka D. Isolated cardiac sarcoidosis: Clinical characteristics, diagnosis and treatment. Intern J Cardiol 2015;182:132-140. doi: 10.1016/j.ijcard.2014.12.056.

262. Okada DR, Bravo PE, Vita T, Agarwal V, Osborn MT, Taiqueti T, Skali H, Chareontaitawee P, Dorbala S, Stewart G, Di Carli M, Blankstein R. Isolated cardiac sarcoidosis. A focused review of an under-recognized entity. J Nuclear Cardiology 2018;25(4):1136-1146. doi: 10.1007/s12350-016_0658-1.

263. Kandolin R, Lehtonen J, Airaksinen J, Vihinen T, Miettinen H, Ylitalo K, Kaikkonen K, Tuohinen S, Haataja P, Kerola T, Kokkonen J, Pelkonen M, Pietila-Effati P, Utrianen S, Kupari M. Cardiac sarcoidosis. Epidemiology, characteristics, and outcome over 25 years in a nationwide study. Circulation 2015;131:624-32. doi:10.1161/CIRCULATIONAHA.114.011522.

264. Simonen P, Lehtonen J, Kandolin R, Schildt J, Marjasuo S, Miettinen H, Airaksinen J, Vihinen T, Tuohinen S, Haataja T, Kupari M. F-18-fluorodeoxyglucose Positron Emission Tomography-guided sampling of mediastinal lymph nodes in the diagnosis of cardiac sarcoidosis. Am J Cardiol 2015;116:1581-1585. doi:10.1016/j.amjcard.2015.08.025.

265. Otsuka K, Terasaki F, Eishi Y, Shimomura H, Ogura Y, Horii T, Isomura T, Suma H, Kitaura Y. Cardiac sarcoidosis underlies idiopathic dilated cardiomyopathy - Importance of mediastinal lymphadenopathy in differential diagnosis. Circ J 2007;71:1937-1941.

266. Revised Guidelines for Diagnosis Cardiac Sarcoidosis 2006 (Japanese Society of Sarcoidosis and Other Granulomatous Disorders) criteria Japanese Ministery of Health 2006 Japanese Ministery Health Welfare. Diagnostic standard and guidelines for sarcoidosis. Jpn J Sarcoidosis Granulomatous Disord (Jpn) 2007;27:89-102.

267. Steger A, Weichert W, Ibrahim T, Rischpler C. Isolated cardiac sarcoidosis: the crucial role of multimodal imaging with positron emission tomography/magnetic resonance imaging in diagnosis and therapy surveillance. Eur Heart J 2018;39(6):488. doi: 10.1093/eurheartj/ehx689.

268. Schwab J, Fessele K, Bastian D, Ficker JH, Papadopoulos T, Lell M, Pauschinger M. CMR imaging for follow up of isolated cardiac sarcoidosis with extensive biventricular involvement. Int J Cardiol 2016;221:777-779. doi: 10.1016/j.ijcard.2016.06.295 
269. Ehl NF, Maeder MT, Joerg L. Isolated cardiac sarcoidosis: critical role of cardiac MRI for diagnosis and management. Eur Heart J 2015;36(48):3434. doi: 10.1093/eurheartj/ehu513.

270. White J, Sutton T, Kerr A. Isolated primary cardiac sarcoidosis: MRI diagnosis and monitoring of treatment response with cardiac enzymes. Circ Heart Fail 2010;3(6):e28-29. doi: 10.1161/cirheartfailure.110.939686.

271. Galati G, Leone O, Rapezzi C. The difficult diagnosis of isolated cardiac sarcoidosis: usefulness of an integrated MRI and PET approach. Heart 2014;100(1):89-90. doi: 10.1136/heartjnl-2013-304237.

272. Juneau D, Nery P, Russo J, de Kemp RA, Leung E, Beanlands RSB, Birnie DH. How common is isolated cardiac sarcoidosis? Extra-cardiac and cardiac findings on clinical examination and whole-body $18 \mathrm{~F}-$ fluorodeoxyglucose positron emission tomography. Int J Cardiol 2018;253:189-193. doi: 10.1016/j.ijcard.2017.09.204

273. Schindler TH, Valenta I. Isolated cardiac sarcoidosis- A rare disease entity? Int J Cardiol 2018;253:194-195. https://doi.org/10/1016/j.ijcard2017.11.005

274. Thachil A, Christopher J, Sastry BKS, Reddy KN, Tourani VK, Hassan A, Raju BS, Narasimhan C. Monomorphic ventricular tachycardia and mediastinal adenopathy due to granulomatous infiltration in patients with preserved ventricular function. J Am Coll Cardiol 2011;58:48-55. doi: 10.1016/j.jacc.2011.02.044.

275. Tuominen H, Haarala A, Tikkakoski A, Kähönen M, Nikus K, Sipilä K. 18-FDG-PET in a patient cohort suspected for cardiac sarcoidosis: Right ventricular uptake is associated with pathological uptake in mediastinal lymphe nodes. J Nucl Cardiol 2018 May 2. doi: 10.1007/s12350-018-1291-y. [Epub ahead of print]

276. Tahara N, Bekki M, Sugiyama Y, Tahara A, Fukumoto Y. Importance of extracardiac FDG uptake to diagnose cardiac sarcoidosis. J Nucl Cardiol 2018 Sept 5 doi: 10.1007/s12350-018-1425-2. [Epub ahead of print]

277. Hiraga H, Yuwai K, Hiroe M. Guideline for the diagnosis of cardiac sarcoidosis: study report on diffuse pulmonary diseases from the Japanese Ministery of Health and Welfare. 1993;23-24.

278. A joint procedural position statement on imaging in cardiac sarcoidosis: from the Cardiovascular and Inflammation \& Infection Commitees of the European Association of Nuclear Medicine, the European Association of Cardiovascular Imaging, and the American Society of Nuclear Cardiology. Eur Heart J CVImaging 2017;18:1073-1089. doi:10.1093/ehjcvi/jex146

279. Ishida Y, Yoshinaga K, Miyagawa M, Moroi M, Kondoh C, Kiso K, Kumita S. Recommendations for 18Ffluodeoxyglucose positron emission tomography imaging for cardiac sarcoidosis: Japanese Society of Nuclear Cardiology Recommendations. Ann Nucl Med 2014;28:393-403. doi: 10.1007/s12149-014-0806-0.

280. Mehta D, Willner JM, Akhrass PR. Atrial fibrillation in cardiac sarcoidosis. J Atrial Fib 2015;8(4):1288. doi: 10.4022/jafrib.1288

281. Viles-Gonzalez JF, Pastori L, Fischer A, Wisnivesky JP, Goldman MG, Mehta D. Supraventricular arrhythmias in patients with cardiac sarcoidosis. Chest 2013;143(4):1085-1090.

282. Bhaskaran A, Kumar S, Kizana E, Thomas SP, Chik WWB. Multimodality imaging, electrophysiologic, electroanatomic, and histopathologic characterization of atrial sarcoidosis presenting with sinus arrest and reentrant right atrial flutter. HeartRhythm Case Rep 2018 Aug 16;4(10):469-474. doi: 10.1016/j.hrcr.2018.06.014

283. Spence S, Pena E, Thornhill RE, Nery PB, Birnie DH. Bi-atrial fibrosis detected using three-dimensional late gadolinium enhancement magnetic resonance imaging in a patient with cardiac sarcoidosis. Oxf Med Case Reports 2018 May 16;2018(5):omy016. doi:10.1093/omcr/omy016. eCollection 2018 May.

284. Kowallick JT, Kutty S, Edelmann F, Chiribiri A, Villa A, Steinmetz M, Sohns JM, Staab W, Bettencourt N, Unterberg-Buchwald C, Hasenfuß G, Lotz J, Schuster A. Quantification of left atrial strain and strain rate using Cardiovascular Magnetic Resonance myocardial feature tracking: a feasibility study. J Cardiovasc Magn Res 2014, 16:60 doi:10.1186/s12968-014-0060-6

285. Samar HY, Thompson DV, Doyle M, Williams RB, Yamrozik JA, Reddy ST, Shah M, Biederman RJ. Sarcoidosis; is it confined to just the LV? An RV LGE study. From 17th Annual SCMR Scientific Sessions, New Orleans, LA, USA. 16-19 January 2014.

286. Valsangiocomo Buechel ER, Mertens L. Imaging the right heart: the use of integrated multimodality imaging. Eur Heart J 2012;33:949-960. doi:10.1093/eurheartj/ehr490.

287. van de Veerdonck MC, Marcus JT, Bogaard HJ, Vonk Noordegraaf A. State of the art: advanced imaging of the right ventricle and pulmonary circulation in humans (2013 Grover Conference series). Pulm Circ 2014;4(2):158-168. doi: 10.1086/675978.

288. Maceira AM, Prasad SK, Khan M, Pennell DJ. Reference right ventricular systolic and diastolic function normalized to age, gender and body surface area from steady-state free precession cardiovascular magnetic resonance. Eur Heart J. 2006;27:2879-2888. 
289. Nadel J, Lancefield T, Voskoboinik A, Taylor AJ. Late gadolinium enhancement identified with cardiac magnetic resonance imaging in sarcoidosis patients is associated with long-term ventricular arrhythmia and sudden cardiac death. Eur Heart J Cardiovasc Imaging 2015;16:634-41. doi: 10.1093/ehjci/jeu294.

290. Maceira AM, Prasad SK, Khan M, Pennell DJ. Reference right ventricular systolic and diastolic function normalized to age, gender and body surface area from steady-state free precession cardiovascular magnetic resonance. Eur Heart J. 2006;27:2879-2888.

291. Rudski LG, Lai WW, Afilalo J, Hua L, Handschumacher MD, Chandrasekaran K, Solomon SD, Louie EK, Schiller NB. Guidelines for the echocardiographic assessment of the right heart in adults: a report from the American Society of Echocardiography endorsed by the European Association of Echocardiography, a registered branch of the European Society of Cardiology, and the Canadian Society of Echocardiography. J Am Soc Echocardiogr 2010;23:685-713. doi: 10.1016/j.echo.2010.05.010.

292. Gulati A, Ismail TF, Jabbour A, Alpendurada F, Guha K, Ismail NA, Jabbour A, Alpendurada F, Guha K, Ismail NA, Raza S, Khwaja J, Brown TDH, Morarji K, E Liodakis, Roughton M, Wage R, Pakrashi PC, Sharma R, Carpenter JP, Cook SA, Cowie MR, Assomull RG, Pennell DJ, Prasad SK. The prevalence and prognostic significance of right ventricular systolic dysfunction in non-ischemic dilated cardiomyopathy. Circulation 2013;128(15):1623-1636. doi: 10.1161/CIRCULATIONAHA.113.002518.

293. Epstein AE, DiMarco JP, Ellenbogen KA, Estes NA $3^{\text {rd }}$, Freedman RA, Gettes LS, Gillinov AM, Gregoratos G, Hammill SC, Hayes DL, Hlatky MA, Newby LK, Page RL, Schoenfeld MH, Silka MJ, Stevenson LW, Sweeney MO, Smith SC Jr, Jacobs AK, Adams CD, Anderson JL, Buller CE, Creager MA, Ettinger SM, Faxon DP, Halperin JL, Hiratzka LF, Hunt SA, Krumholz HM, Kushner FG, Lytle BW, Nishimura RA, Ornato JP, Page RL, Riegel B, Tarkington LG, Yancy CW. American College of Cardiology/American Heart Association Task Force on Practice Guidelines (Writing Committee to Revise the ACC/AHA/NASPE 2002 Guideline Update for Implantation of Cardiac Pacemakers and Antiarrhythmia Devices); American Association for Thoracic Surgery; Society of Thoracic Surgeons. ACC/AHA/HRS 2008 guidelines for device-based therapy of cardiac rhythm abnormalities. Circulation 2008;117:e350-e408. doi:10.1016/j.jacc.2008.02.032.

294. Oakley L, Prahl J, Daheshia M, Price G, Boswell G, Alexander S. Diagnosis of right ventricular cardiac sarcoidosis with cardiac magnetic resonance in a patient presenting with ventricular tachycardia. Mil Med 2013;178(2): e265-270. doi: 10.7205/MILMED-D-12-00288.

295. Ueberham L, Paetsch I, Jahnke C, Klingel K, Dinov B. Right ventricular thickening and extensive late gadolinium enhancement in a patient with rare case of isolated cardiac sarcoidosis and initially negative biopsy. Eur Heart J Cardiovasc Imaging 2017 Dec 1;18(12):1427-1428. doi: 10.1093/ehjci/jex226

296. Birnie D, Ha A, Kron J. Which patients with cardiac sarcoidosis should receive implantable cardioverter defibrillator. Circ Arrhythm Electrophys 2018;11:e006685. doi: 10.1161/circep.118.006685

297. Wicks EC, Menezes LJ, Barnes A, Mohiddin SA, Sekhri N, Porter JC, Booth HL, Garrett E, Patel RS, Pavlou M, Groves AM, Elliott P. Diagnostic accuracy and prognostic value of simutaneous hybrid 18Ffluorodeoxyglucose positron emission tomography/magnetic resonance imaging in cardiac sarcoidosis. Eur Heart J Cardiovasc Imaging 2018 Jul 1;19(7):757-767. doi: 10.1093/ehjci/jex340.

298. Murtagh G, Laffin LJ, Beshai JF, Maffessanti F, Bonham CA, Patel AV, Ju Z, Addetia K, Mor-Avi V, Moss JD, Hogarth DK, Sweiss NJ, Lang RM, Patel AR. Prognosis in myocardial damage in sarcoidosis patients with preserved left ventricular ejection fraction. Risk stratification using cardiac magnetic resonance. Circ Cardiovasc Imag 2016;9:e003738. doi: 10.1161/CIRCIMAGING.115.003738.

299. Roberts WC, Chung MS, Ko JM, Capehart JE, Hall SA. Morphologic features of cardiac sarcoidosis in native heart of patients having cardiac transplantation. Am J Cardiol 2014;113:706-12. doi: 10.1016/j.amjcard.2013.11.015.

300. Bagwan IN, Hooper LVB, Shepard MN. Cardiac sarcoidosis and sudden death. The heart may look normal or mimic other cardiomyopathies. Virchow Arch 2011;458:671-78. doi: 10.1007/s00428-010-1003-8.

301. Ekström K, Lehtonen J, Hänninen H, Kandolin R, Kivistö S, Kupari M. Magnetic resonance imaging as a predictor of survival free of life-threatening arrhythmias and transplantation in cardiac sarcoidosis. $\mathrm{J} \mathrm{Am}$ Heart Assoc 2016;5:e003040. doi: 10.1161/JAHA.115.003040.

302. Swift AJ, Rajaram S, Capener D, Elliott C, Condliffe R, Wild JM, Kiely DJ. LGE Patterns in pulmonary hypertension do not impact overall mortality. J Am Coll Cardiol Img 2014;7:1209-1217. doi: 10.1016/j.jcmg.2014.08.014.

303. Baughman RP, Engel PJ, Nathan S. Pulmonary hypertension in sarcoidosis. Clin Chest Med 2015;36:703714. doi: 10.1016/j.ccm.2015.08.011. 
304. Gulati A, Ismail TF, Jabbour A, Alpendurada F, Guha K, Ismail NA, Jabbour A, Alpendurada F, Guha K, Ismail NA, Raza S, Khwaja J, Brown TDH, Morarji K, E Liodakis, Roughton M, Wage R, Pakrashi PC, Sharma R, Carpenter JP, Cook SA, Cowie MR, Assomull RG, Pennell DJ, Prasad SK. The prevalence and prognostic significance of right ventricular systolic dysfunction in nonischemic dilated cardiomyopathy. Circulation 2013;128(15):1623-1633. doi: 10.1161/circulationaha.113.002518

305. Philips B, Madhavan S, James CA, te Riele A, Murray B, Tichnell C, Bhonsale A, Nazarian S, Judge DP, Calkins H, Tandri H, Cheng A. Arrhythmogenic Right Ventricular Dysplasia/ Cardiomyopathy and Cardiac Sarcoidosis. Distinguishing features when the diagnosis is unclear. Circ Arrhythm Electrophysiol 2014;7:230236. doi: 10.1161/CIRCEP.113.000932.

306. Sato T, Tsujino I, Ohira H, Oyama-Manabe N, Ito YM, Noguchi T, Yamada A, Ikeda D, Watanabe T, Nishimura M. Paradoxal interventricular septal motion as a major determinant of late gadolinium enhancement in ventricular insertion points in pulmonary hypertension. PLOs one 2013;8(6):e66724. doi: 10.1371/journal.pone.0066724.

307. Gomez A, Bialostozky D, Zajarias A, Santos E, Palomar A, Martinez ML, Sandoval J. Right ventricular ischemia in patients with primary pulmonary hypertension. J Am Coll Cardiol 2001;38(4):1137-41.

308. Kuribayashi T, Roberts WC. Myocardial disarray at junction of ventricular septum and left and right ventricular free walls in hypertrophic cardiomyopathy. Am J Cardiol 1992;70:1333-40.

309. Babu-Narayan SV, Kilner PJ, Li W, Moon JC, Goktekin O, Davlouros PA, Khan M, Ho SY, Pennell DJ, Gatzoulis MA. Ventricular fibrosis suggested by cardiovascular magnetic resonance in adults with repaired tetralogy of Fallot and its relationship to adverse markers of clinical outcome. Circulation 2006; 113:405413. doi:10.1161/CIRCULATIONAHA.105.548727.

310. McCann GP, Beek AM, Vonk-Noordegraaf A, van Rossum AC. Delayed contrast-enhanced magnetic resonance imaging in pulmonary arterial hypertension. Circulation 2005;112:e268. doi: 10.1161/CIRCULATIONAHA.104.512848.

311. Zorzi A, Perazzolo Marra M, Rigato I, De Lazzari M, Susana A, Niero A, Pilichou K, Migliore F, Rizzo S, Giorgi B, De Conti G, Sarto P, Serratosa L, Patrizi G, De Maria E, Pelliccia A, Basso C, Schiavon M, Bauce B, Iliceto S, Thiene G, Corrado D. Non-Ischemic left ventricular scar as a substrate of life-threatening ventricular arrhythmias and sudden cardiac death in competitive athletes. Circ Arrhythm Electrophysiol 2016:9:e004229. doi: 10.1161/CIRCEP.116.004229.

312. Mavrogeni S, Kouranos V, Sfikakis PP, Bratis K, Kitas GD, Manali E, Perros E, Vlasis K, Rapti A, Koulouris N, van Wijk K, Hautemann D, Reiber JHC, Kolovou G, Tzelepis GE, Gialafos E. Myocardial stress perfusion-fibrosis imaging pattern in sarcoidosis, assessed by cardiovascular magnetic resonance imaging. Int J Cardiol 2014;172(2):501-503. doi:10.1016/j.ijcard.2014.01.006

313. Kruse MJ, Kovell L, Kasper EK, Pomper MG, Moller DR, Solnes L, Chen ES, Schindler TH. Myocardial blood flow and inflammatory cardiac sarcoidosis. JACC Cardiovasc Imaging 2017;10(20:157-167. doi: 10.1016/j.jcmg.2016.09.023

314. Tellier P, Paycha F, Antony I, Nitenberg A, Valeyre D, Foult JM, Battesti JP. Reversibility by dipyridamole of thallium-201 myocardial scan defects in patients with sarcoidosis. Am J Med 1988;85(2):189-193.

315. Hunold P, Schlosser T, Vogt FM, Eggebrecht H, Schmermund A, Bruder O, Schüler WO, Barkhausen J.Myocardial late enhancement in contrast-enhanced cardiac MRI: distinction between infarction scar and non-infarction-related disease. AJR Am J Roentgenol 2005;184(5):1420-6. doi: 10.2214/ajr.184.5.01841420.

316. Belloni E, De Cobelli F, Esposito A, Mellone R, Perseghin G, Canu T, Del Maschio A. MRI of cardiomyopathy. AJR Am J Roentgenol 2008;191(6):1702-1710. doi: 10.2214/AJR.07.3997.

317. Cummings KW, Bhalla S, Javidan-Nejad C, Bierhals AJ, Gutierrez FR, Woodard PK. A pattern-based approach to assessment of delayed enhancement in nonischemic cardiomyopathy at MR imaging. Radiographics 2009;29(1):89-103. doi: 10.1148/rg.291085052.

318. Patel AR, Kramer CM. Role of cardiac magnetic resonance imaging in the diagnosis and prognosis of nonischemic cardiomyopathy. JACC Cardiovasc Imaging 2017;10:1180-1193. doi: 10.1016/j.jcmg.2017.08.005.

319. Porcari A, De Angelis G, Romani S, Paldino A, Artico J, Cannata A, Gentile P, Pinamonti B, Merlo M, Sinagra G. Current imaging strategies for dilated cardiomyopathy: a comparison of imaging techniques. Expert Rev Cardiovasc Ther 2019;17(1):53-63. doi: 10.1080/14779072.2019.1550719. [Epub ahead of print]

320. Marcu CB, Nijveldt R, Beek AM, van Rossum AC. Delayed contrast enhancement magnetic resonance imaging for the assessment of cardiac disease. Heart, Lung and Circulation 2007;16:70-78. doi: 10.1016/j.hlc.2006.09.006 
321. Sechtem A, Mahrholdt $H$, Vogelsberg H. Cardiac magnetic resonance in myocardiual disease. Heart 2007;93:1520-1527. doi: 10.1136/hrt.2005.067355

322. Matoh F, Satoh H, Shiraki K, Odagiri K, Saitoh T, Urushida T, Katoh H, Takehara Y, Sakahara H, Hayashi $H$. The usefulness of delayed enhanced magnetic imaging for diagnosis and evaluation of cardiac function in patients with cardiac sarcoidosis. J Cardiol 2008;51:179-188. doi: 10.1016/j.jjcc.2008.03.002.

323. Jeudy J, Burke AP, White CS, Kramer GBG, Frazier AA. Cardiac sarcoidosis: The challenge of radiologic and pathologic correlation. Radiographics 2015;35:657-679. doi: 10.1148/rg.2015140247.

324. Watanabe E, Kimura F, Nakajima T, Hiroe M, Kasai Y, Nagata M, Kawana M, Hagiwara N. Late gadolinium enhancement in cardiac sarcoidosis: characteristic magnetic resonance findings and relationship with left ventricular function. J Thorac Imaging 2013:28:60-66. doi: 10.1097/RTI.0b013e3182761830.

325. Sano M, Satoh H, Suwa K, Saotome M, Urushida T, Katoh H, Hayashi H, Saitoh T. Intra-cardiac distribution of late gadolinium enhancement in cardiac sarcoidosis and dilated cardiomyopathy. World J Cardiol 2016;8(9):469-503.

326. McCann GP, Gan CT, Beek Am, Niessen HW, Vonk Noordegraaf A, van Rossum AC. Extent of MRI Delayed Enhancement of Myocardial Mass Is Related to Right Ventricular Dysfunction in Pulmonary Artery Hypertension. AJR Am J Roentgenol 2007;188:349-355.

327. Smedema JP, van Geuns RJ, Truter R, Mayosi BM, Crijns HJGM. Contrast-Enhanced Cardiac Magnetic Resonance: Distinction between cardiac sarcoidosis and infarction scar. Sarc Vasc Diff Lung Dis 2017;34:307-314. http://www.mattioli1885journals.com/index.php/sarcoidosis/article/view/5715

328. Fussner LA, Karlstedt E, Hodge DO, Fine NM, Kalra S, Carmona EM, Utz JP, Isaac DL, Cooper LT. Management and outcomes of cardiac sarcoidosis: a 20-year experience in two tertiary care centres. Eur J Heart Fail 2018 Oct 31. doi: 10.1002/ejhf.1319 [Epub ahead of print]

329. Ghanizada M, Rossing K, Bundgaard H, Gustafsson F. Clinical presentation, management and prognosis of patients with cardiac sarcoidosis. Dan Med J 2018;65(4). Pii: A5462.

330. Bruder O, Mahrholdt H. CMR Imaging predicts death and other adverse events in suspected cardiac sarcoidosis. J Am Coll Cardiol Img 2013;6:501-11. doi: 10.1016/j.jcmg.2012.10.021.

331. Okada DR, Smith J, Derakhshan A, Gowani Z, Misra S, Berger RD, Calkins H, Tandri H, Chrispin P. Ventricular arrhythmias in cardiac sarcoidosis. Circulation 2018;138:1253-1264. doi: 10.1161/circulationaha.118.034687

332. Nordenswan HK, Lehtonen J, Ekström K, Kandolin R, Simonen P, Mäyranpää P, Vihinen T, Miettinen H, Kaikkonen K, Haataja P, Kerola T, Rissanen TT, Kokkonen J, Alatalo A, Pietilä-Effati P, Utriainen S, Kupari M. Outcome of cardiac sarcoidosis presenting with high-grade atrioventricular block. Circ Arrhythm Electrophys 2018;11:e006145. doi: 10.1161/ circep.117.006145

333. Neilan TG, Coelho-Filho OR, Danik SB, Shah RV, Dodson JA, Verdini DJ, Tokuda M, Daly CA, Tedrow UB, Stevenson WG, Jerosh-Herold M, Ghoshhajra BB, Kwong RY. CMR quantification of myocardial scar provides additive prognostic information in nonischemic cardiomyopathy. JACC Cardiovasc Imaging 2013;6(9):944-954. doi: 10.1016/j.jcmg.2013.05.013

334. Ise T, Hasegawa T, Morita Y, Yamada N, Funada A, Takahama H, Amaki M, Kanzaki H, Okamura H, Kamakura s, Shimizu W, Aanzai T, Kitakaze M. Extensive late gadolinium enhancement on cardiovascular magnetic resonance predicts adverse outcomes and lack of improvement in LV function after steroid therapy in cardiac sarcoidosis. Heart 2014;100:1165-1172. doi: 10.1136/heartjnl-2013-305187.

335. Sobue Y, Harada M, Kohsikawa M, Ischikawa T, Yamamoto M, Okuda K, Kato Y, Sarai M, Watanabe A, Ozaki Y. QRS-based assessment of myocardial damage and adverse events associated with cardiac sarcoidosis. Heart Rhythm 2015;12:2499-2507. doi: 10.1016/j.hrthm.215.09.008.

336. Yasuda M, Iwanaga Y, Kato T, Izumi T, Inuzuka Y, Nakamura T, Miyaji Y, Kawamura T, Ikeguchi S, Inoko M, Kurita T, Miyazaki S. Risk stratification for major adverse cardiac events and ventricular tachyarrhythmia's by cardiac MRI in patients with sarcoidosis. Open Heart 2016;3:e000437. doi:10.1136/openhrt-2016000437

337. Sipilä K, Tuominen H, Haarala A, Tikkakoski A, Kähönen M, Nikus K. Novel ECG parameters are strongly associated with inflammatory ${ }^{18} \mathrm{~F}$-FDG-PET findings in patients with suspected cardiac sarcoidosis. Intern J Cardiol 2017;249:454-460. doi: 10.1016/j.ijcard.2017.07.027.

338. Yodogawa K, Seino Y, Ohara T, Iwasaki Y, Hayashi M, Miyauchi Y, Azuma A, Shimizu W. Prognostic significance of ventricular late potentials in patients with pulmonary sarcoidosis. HeartRhythm 2018;15(6):798-802. doi.org/10.1016/jhrthm.2018.03.013 
339. Nagai T, Kohsaka S, Okuda S, Anzai T, Asano K, Fukuda K. Incidence and prognostic significance of myocardial late gadolinium-enhancement in sarcoidosis patients without cardiac manifestation. Chest 2014;146(4):1064-72. doi: 10.1378/chest.14-0139.

340. Shafee MA, Fukuda K, Wakayama Y, Nakano M, Kondo M, Hasebe Y, Kawana A, Shimokawa H. Delayed enhancement on cardiac magnetic resonance imaging is a poor prognostic factor in patients with cardiac sarcoidosis. J Cardiol 2012;60:448-453. doi: 10.1016/j.jjcc.2012.08.002.

341. Kouranos V,Tzelepis GE, Rapti A, Mavrogeni S, Aggeli K, Douskou M, Prasad S, Koulouris N, Sfikakis P, Wells A, Gialafos E. Complementary role of CMR to conventional screening in the diagnosis and prognosis of cardiac sarcoidosis. JACC CVImaging 2017;10(12):1437-1447. doi: 10.1016/j.jcmg.2016.11.019.

342. Klem I, Weinsaft JW, Bahnson TD, Hegland D, Kim HW, Hayes B, Parker MA, Judd RM, Kim RJ. Assessment of myocardial scarring improves risk stratification in patients evaluated for cardiac defibrillator implantation. J Am Coll Cardiol 2012;60:408-20. doi: 10.1016/j.jacc.2012.02.070.

343. Hena KM, Yip J, Jaber N, Goldfarb D, Fullam K, Cleven K, Moir W, Zeig-Owens R, Webber MP, Spevack DM, Judson MA, Maier L, Krumerman A, Aizer A, Spivack SD, Berman J, Aldrich TK, Prezant DJ. Clinical course of sarcoidosis in World Trade Center-exposed firefighters. Chest 2018;153(1):114-123. doi: 10.1016/j.chest.2017.10.014

344. Nery PB, Beanlands RS, Nair GM, Green M, Yang J, McArdle BA, Davis D, Ohira H, Gollob MH, Leung E, Healy JS, Birnie DH. Atrioventricular block as the initial manifestation of cardiac sarcoidosis in middleaged adults. J Cardiovasc Electrophys 2014;25:875-881. doi: 10.111/jce.12401

345. Forotan H, Rowe MK, Korczyk D, Kaye G. Cardiac sarcoidosis, left ventricular impairment and chronic right ventricular pacing: Pacing or pathology? Heart Lung Circ 2017;26(11):1175-1182. doi: 10.1016/j.hlc.2017.03.167

346. Yufu K, Kondo H, Shinohara T, Kawano K, Ishii Y, Miyoshi M, Imamura T, Saito S, Okada N, Akioka H, Teshima Y, Nakagawa M, Takahashi N. Outcome of patients with cardiac sarcoidosis who received cardiac synchronization therapy: Comparison with dilated cardiomyopathy patients. J Cardiovasc Electrophys 2017;28(2):177-181. doi: 10.1111/jce.13119.

347. Patel D, Trulock KM, Toro S, Grimaldi A, Gonzalez M, Moennich LA, Gorodeski EZ, Joyce E, Niebauer M, Wilkoff BL, Varma N, Rickard JW. Effect of cardiac resynchronization therapy on left ventricular remodeling in patients with cardiac sarcoidosis. Am J Cardiol 2018;00:1-5. doi: 10.1016/j.amjcard.2018.09.044. [Epub ahead of print]

348. Andreu D, Ortiz-Pérez JT, Boussy T, Fernandez-Armenta J, de Caralt TM, Perea RJ, Prat-Gonzalez S, Mont L, Brugada J, Berruezo A. Usefulness of contrast-enhanced cardiac magnetic resonance in identifying the ventricular arrhythmia substrate and the approach needed for ablation. Eur Heart J 2014;23:1316-1326. doi: 10.1093/eurheartj/ehj510.

349. Bogun FM, Desjardins B, Good E, Gupta S, Crawford T, Oral H, Ebinger M, Pelosi F, Chugh A, Jongnarangsin K, Morady F. Delayed-Enhanced Magnetic Resonance Imaging in non-ischemic cardiomyopathy. Utility for identifying the ventricular arrhythmia substrate. J Am Coll Cardiol 2009;53:1138-1145. doi: 10.1016/j.jacc.2008.11.052

350. Siontis KC, Kim HM, Dabbagh S, Latchamsetty R, Stojanovska J, Jongnarangsin K, Morady F, Bogun FM. Association of preprocedural cardiac magnetic resonance imaging with outcomes of ventricular tachycardia ablation in patients with idiopathic dilated cardiomyopathy. Heart Rhythm 2017;14(10):1487-1493. doi: 10.1016/j.hrthm.2017.06.003. doi: 10.1016/j.hrthm.2017.06.003.

351. Nazarian S, Bluemke DA, Lardo AC, Zviman MM, Watkins SP, Dickfield TL, Meininger GR, Roguin A, Calkins H, Tomaselli GF, Weis RG, Berger RD, Lima JA, Halperin HR. Magnetic resonance assessment of the substrate for inducible ventricular tachycardia in nonischemic cardiomyopathy. Circulation 2005;112(18):2821-2825.

352. Nazarian S. Editorial comment. CMR for mapping the missing dimension in ventricular tachycardia ablation. JACC Cardiovasc Imag 2010;3:286-288. doi: 10.1016/j.jcmg.2009.11.007.

353. Andreu D, Ortiz-Pérez JT, Boussy T, Fernández-Armenta J, de Caralt TM, Perea RJ, Prat-González S, Mont L, Brugada J, Berruezo A. Usefulness of contrast-enhanced cardiac magnetic resonance in identifying the ventricular arrhythmia substrate and the approach needed for ablation. Eur Heart J 2014;23:1316-1326. doi: 10.1093/eurheartj/eht510.

354. Nazarian S. Cardiac electrophysiology procedures, know unkowns, and unknown unknowns: the potential of magnetic resonance guidance. JACC Clin Electrophys 2017;3(2):104-106. doi: 10.1016/j.jacep.2016.09.012. 
355. Ipek EG, Nazarian S. Cardiac magnetic resonance for prediction of arrhythmogenic areas. Trends in Cardiovasc Med 2015;25:635-642. doi: 10.1016/j.tcm.2015.02.012.

356. Zghaib T, Ipek EG, Hansford R, Ashikaga H, Berger RD, Marine JE, Spragg DD, Tandri H, Zimmerman SL, Halperin H, Brancato S, Calkins H, Henrikson C, Nazarian S. Standard ablation versus magnetic imaging-guided ablation in the treatment of ventricular tachycardia. Circ Arrhythm Electrophysiol 2018;11(1):e005973. doi: 10.1161/circep.117.005973.

357. Sasaki T, Miller CF, Hansford R, Zipunnikov V, Zviman MM, Marine JE, Spragg D, Cheng A, Tandri H, Sinha S, Kolandaivelu A, Zimmerman SL, Bluemke DA, Tomaselli GF, Berger RD, Halperin HR, Calkins H, Nazarian S. Impact of nonischemic scar features on local ventricular electrograms and scar-related ventricular tachycardia circuits in patients with nonischemic cardiomyopathy. Circ Arrhythm Electrophysiol 2013;6(6):1139-1147. doi: 10.1161/circep.113.000159

358. Mc Ardle BA, Leung E, Ohira H, Cocker MS, de Kemp RA, DaSilva J, Birnie D, Beanlands RS, Nery PB. The role of $\mathrm{F}(18)$-fluorodeoxyglucose positron emission tomography in guiding diagnosis and management in patients with known or suspected cardiac sarcoidosis. J Nuclear Cardiol 2013;20:297-306. doi: 10.1007/s12350-012-9668-9

359. Fleming HA, Bailey SM. The prognosis of sarcoid heart disease in the United Kingdom. Ann N Y Acad Sci 1986;465:543-550. doi: 10.1111/j.1749-6632.

360. Chapelon-Abric C, Sene D, Saadoun D, Cluzel P, Vignaux O, Costedoat-Chalumeau N, Piette JC, Cacoub P. Cardiac sarcoidosis: Diagnosis, therapeutic management, and prognostic management. Arch Cardiovasc Dis 2017;110(8-9):456-465. Pii: S1875-2136(17)30096-7. doi: 10.1016/j.acvd.2016.12.014

361. Zhou Y, Lower EE, Li H, Costae A, Attari M, Baughman RP. Cardiac sarcoidosis: the impact of age and implanted devices on survival. Chest 2017;151(1):139-148. doi: 10.1016/j.chest.2016.08.1457.

362. Kandolin R, Lehtonen J, Kupari M. Cardiac sarcoidosis. J Intern Med 2016;280(1):129-131. doi: 10.111/joim.12498.

363. Vita T, Okada DR, Veillet-Chowdhury M, Bravo PE, Mullins E, Hulten E, Agrawal M, Madan R, Taqeti VR, Steigner M, Skali H, Kwong RY, Stewart GC, Dorbala S, Di Carli MF, Blankstein R. Complementary value of cardiac magenetic resonance imaging and positron emission tomography/computed tomography in the assessment of cardiac sarcoidosis. Circ Cardiovasc Imaging 2018;11:e007030. doi: 10.1161/ circimaging.117.007030.

364. Krumm P, Mangold S, Gatidis S, Nikolaou K, Nensa F, Bamberg F, la Fougere C. Clinical use of cardiac PET/MRI: current-state-of-the-art of potential future applications. Jpn J Radiol 2018;36(5):313-323. doi: 10.1007/s11604-018-0727-2.

365. Wicks EC, Menezes LJ, Barnes A, Mohiddin SA, Sekhri N, Porter JC, Booth HL, Garrett E, Patel RS, Pavlou M, Groves AM, Elliott P. Diagnostic accuracy and prognostic value of simutaneous hybrid 18Ffluorodeoxyglucose positron emission tomography/magnetic resonance imaging in cardiac sarcoidosis. Eur Heart J Cardiovasc Imaging 2018;19(7):757-767. doi: 10.1093/ehjci/jex340.

366. Sgard B, Brillet PY, Bouvry D, Djelbani S, Nunes H, Meune C, Valeyre D, Soussan M. Evaluation of FDG PET combined with cardiac MRI for the diagnosis and therapeutic monitoring of cardiac sarcoidosis. Clin Radiol 2018 Oct 25. doi: 10.1016/j.crad.2018.09.015. [Epub ahead of print]

367. Hamilton JI, Jiang Y, Chen Y, Ma D, Lo W-C, Griswold M, Seiberlich N. MR fingerprinting for rapid quantification of myocardial T1, T2, and proton spin density. Mag Reson Med 2017;77(4):1446-1458. doi: $10.1002 / \mathrm{mrm} .26212$.

368. Unterberg-Buchwald C, Ritter CO, Reupke V, Wilke RN, Stadelmann C, Steinmetz M, Schuster A, Hasenfuß G, Lotz J, Eucker M. Targeted endomyocardial biopsy guided by real-time cardiovascular magnetic resonance. J Cardiovasc Magn Reson 2017 Apr 19;19(1):45. doi: 10.1186/s12968-017-0357-3.

369. Watanabe Y, Nishii T, Shimoyama S, Ito T, Mori S, Kono AK, Takahashi S, Hirata KI, Sugimura K. Focal myocardial damage in cardiac sarcoidosis characterized by strain analysis on magnetic resonance tagged imaging in comparison with Fluorodeoxyglucose Positron Emission Tomography accumulation and magnetic resonance late gadolinium enhancement. J Comput Assist Tomogr 2018;42(4):607-613. doi: 10.1097/RCT.0000000000000733.

370. Chubb H, Williams SE, Whitaker J, Harrison JL, Razavi R, O’Neill M. Cardiac electrophysiology under MRI guidance: an emerging technology. Arrhythm Electrophysiol Rev 2017;6(2):85-93. doi: 10.15420/aer.2017.1.2. 
Chapter 2

371. Mukherjee RK, Whitaker J, Williams SE, Razavi R, O’Neill MD. Magnetic resonance imaging guidance for the optimization of ventricular tachycardia ablation. Europace 2018;20(11):1721-1732. doi: 10.1093/europace/euy040. 
Table 1. Symptoms of cardiac sarcoidosis

1. (aborted) sudden cardiac death may be the first presentation: VT/VF, AVB

2. palpitations: PAF, SVTs, (non-)sustained VTs

3. pre/syncopal episodes: SVTs, AVB, VT/VF, cor pulmonale

4. chest discomfort: compression of intramural coronary arteries by granulomas, pericarditis, rarely coronary vasculitis, pulmonary hypertension

5. dyspnea: arrhythmias, diastolic or systolic heart failure, pericarditis, tamponade, mitral regurgitation, pulmonary hypertension

6. pitting edema lower extremities: systolic or diastolic heart failure, tamponade

VT/VF- ventricular tachycardia/ventricular fibrillation

AVB - atrioventricular block

PAF - paroxysmal atrial fibrillation

SVT-supraventricular tachycardia

VT- ventricular tachycardia 


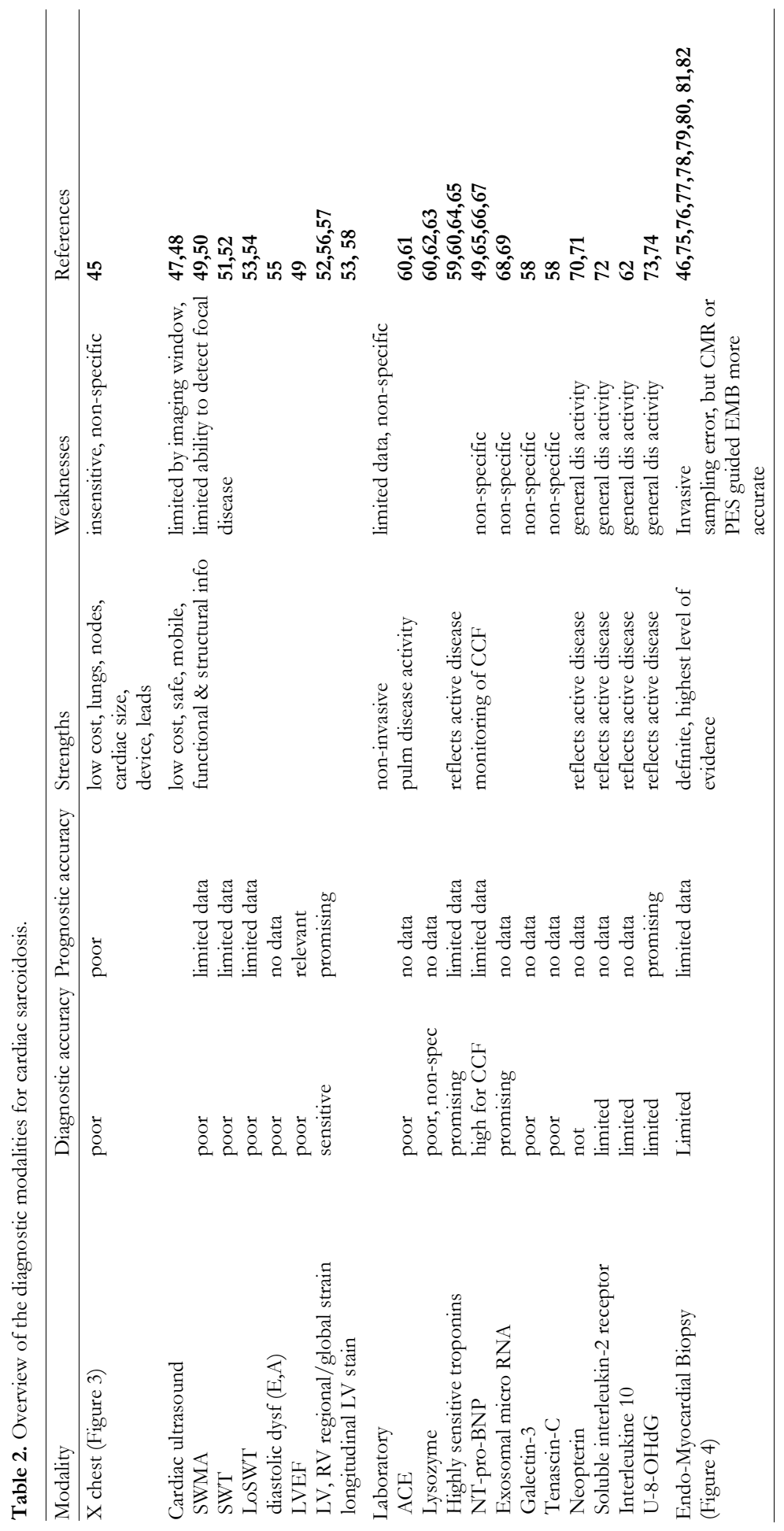




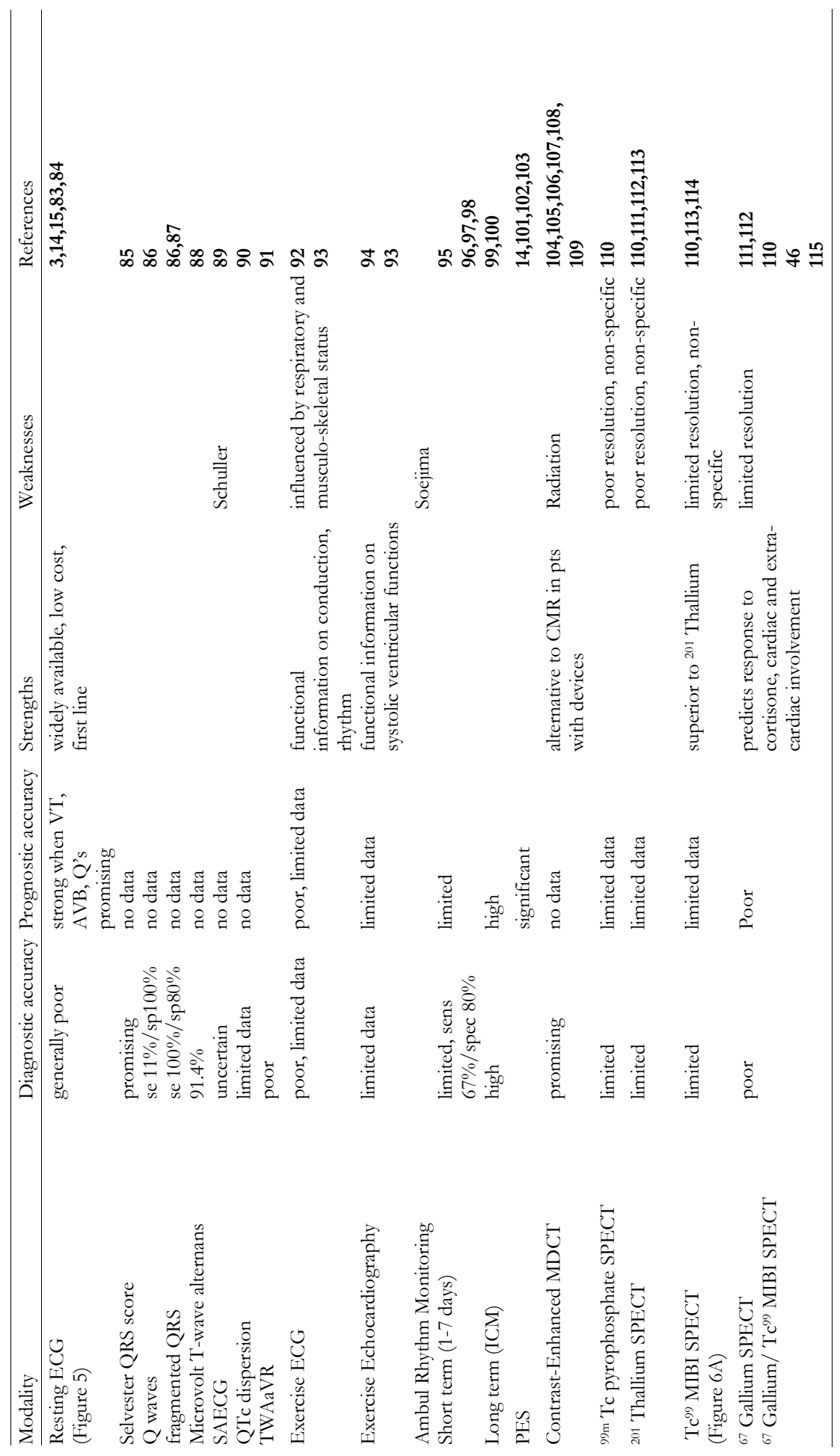




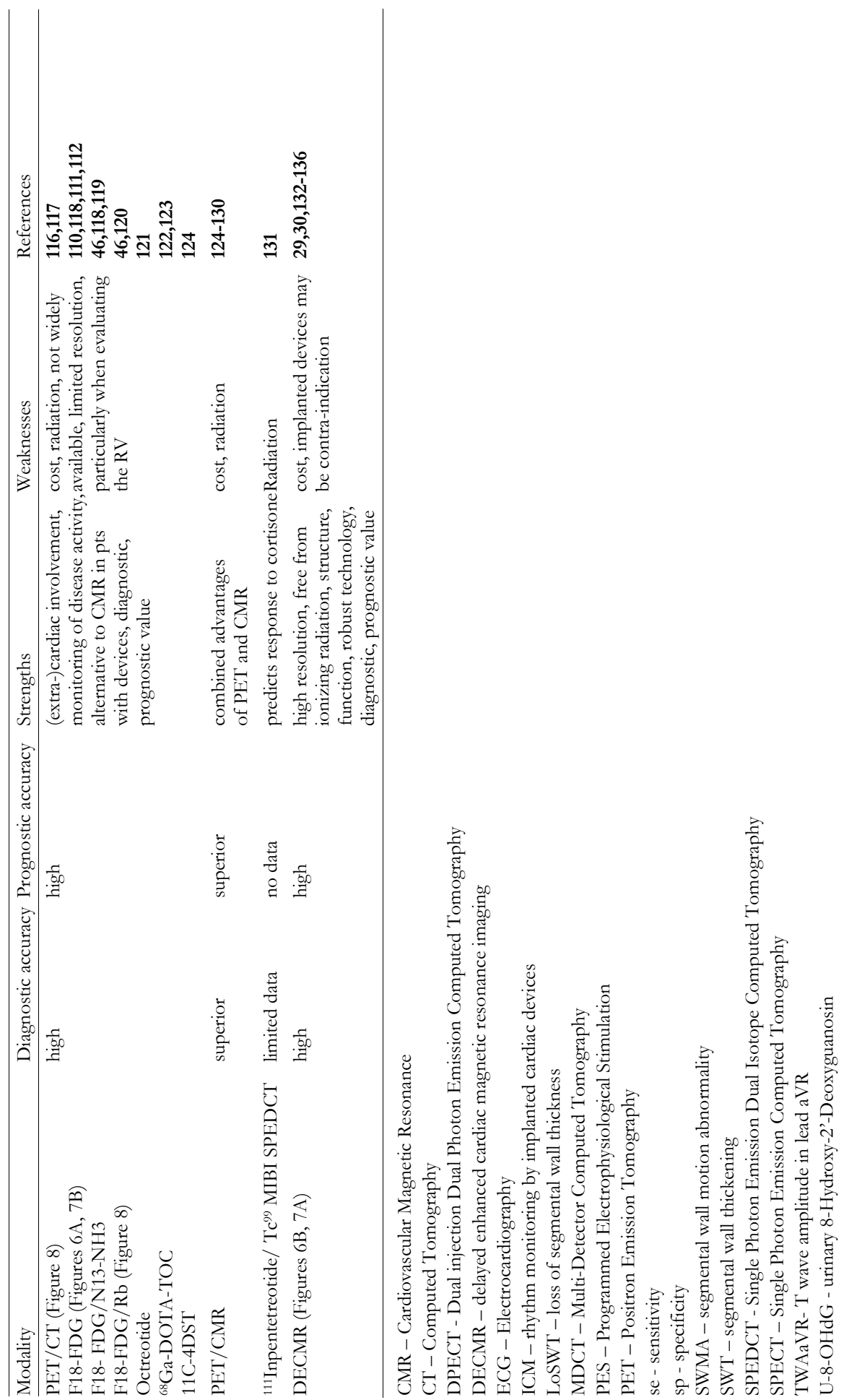




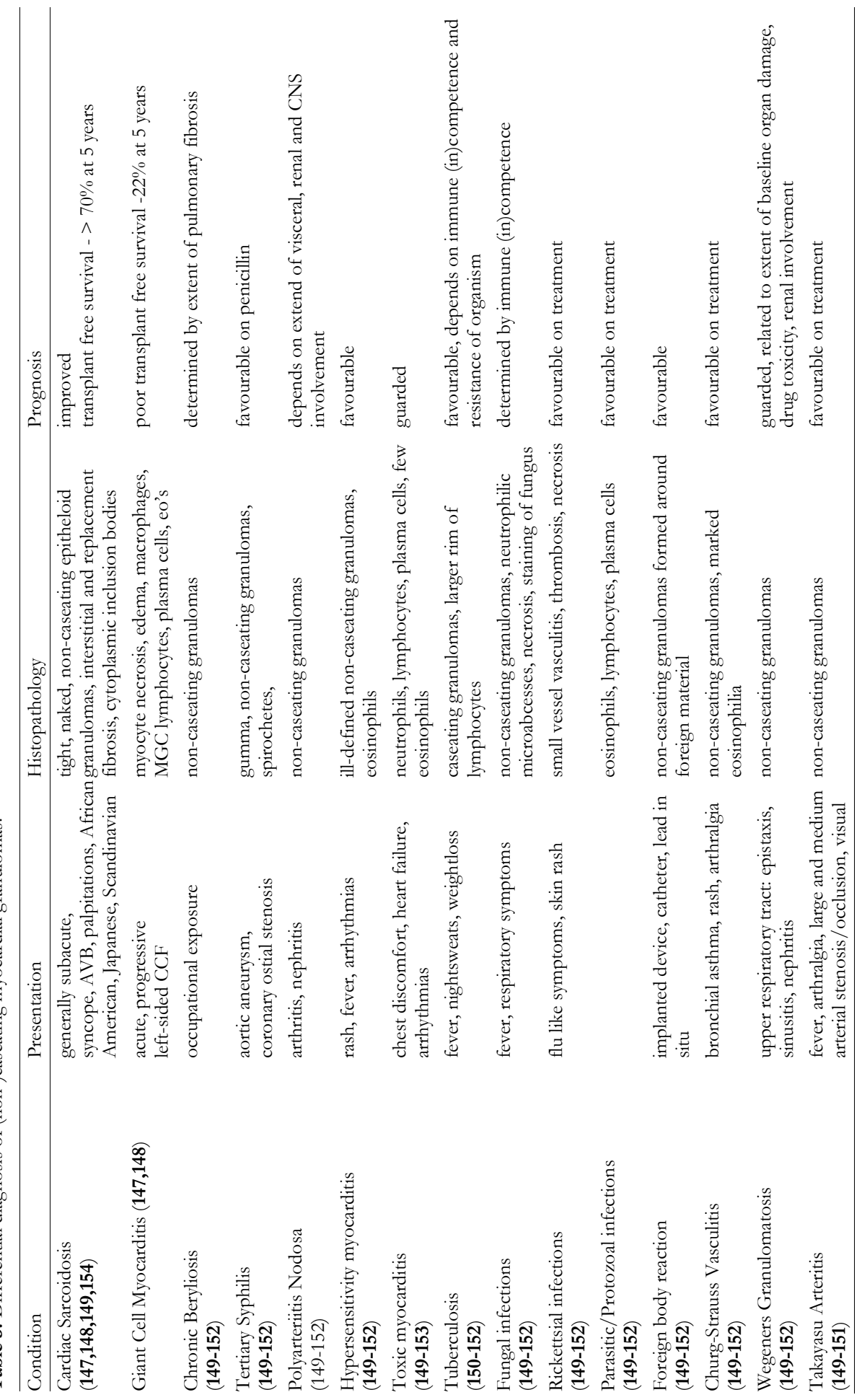


Chapter 2

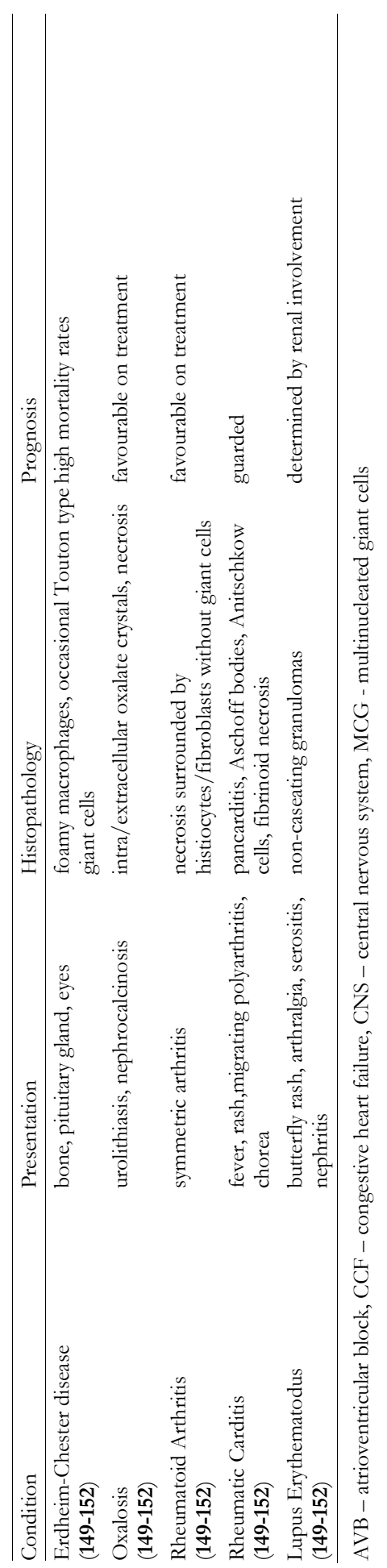




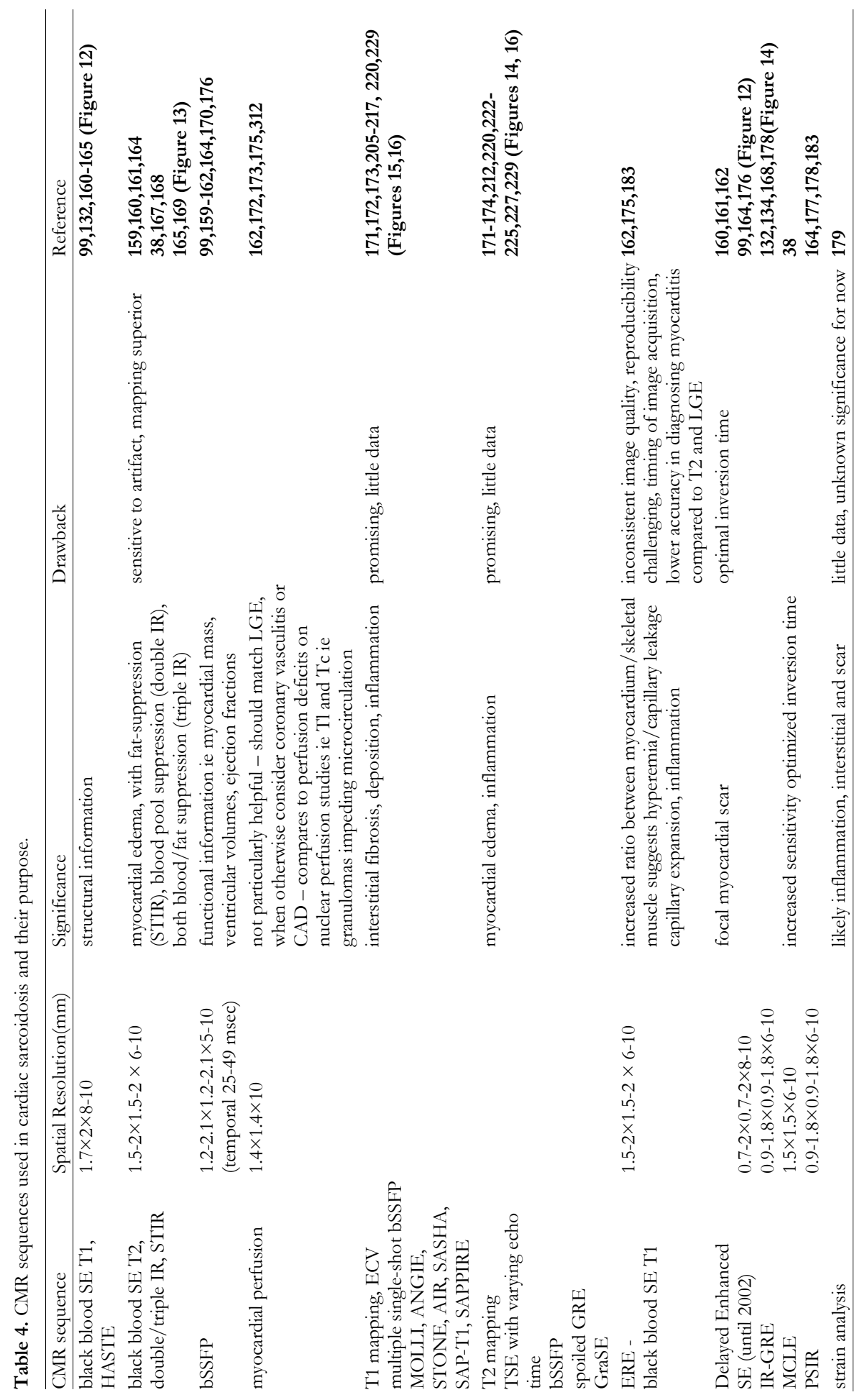


Chapter 2

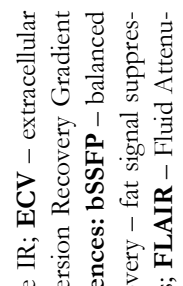

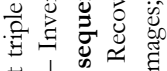
焉 10 品

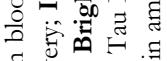
递苟过 品

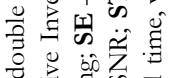

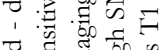

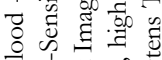

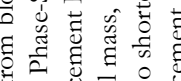

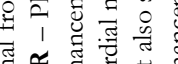

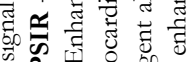

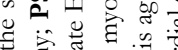
等

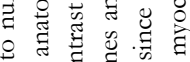
总 8

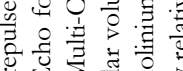

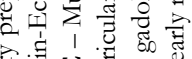

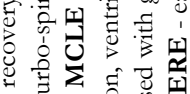

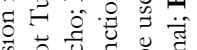

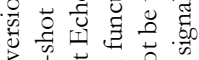

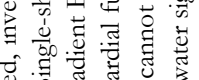

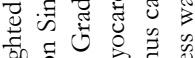

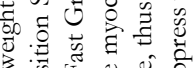

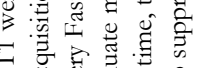

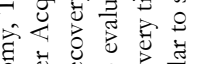

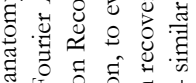

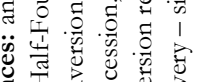

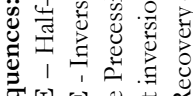
政四 田

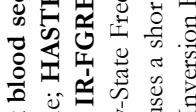

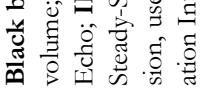

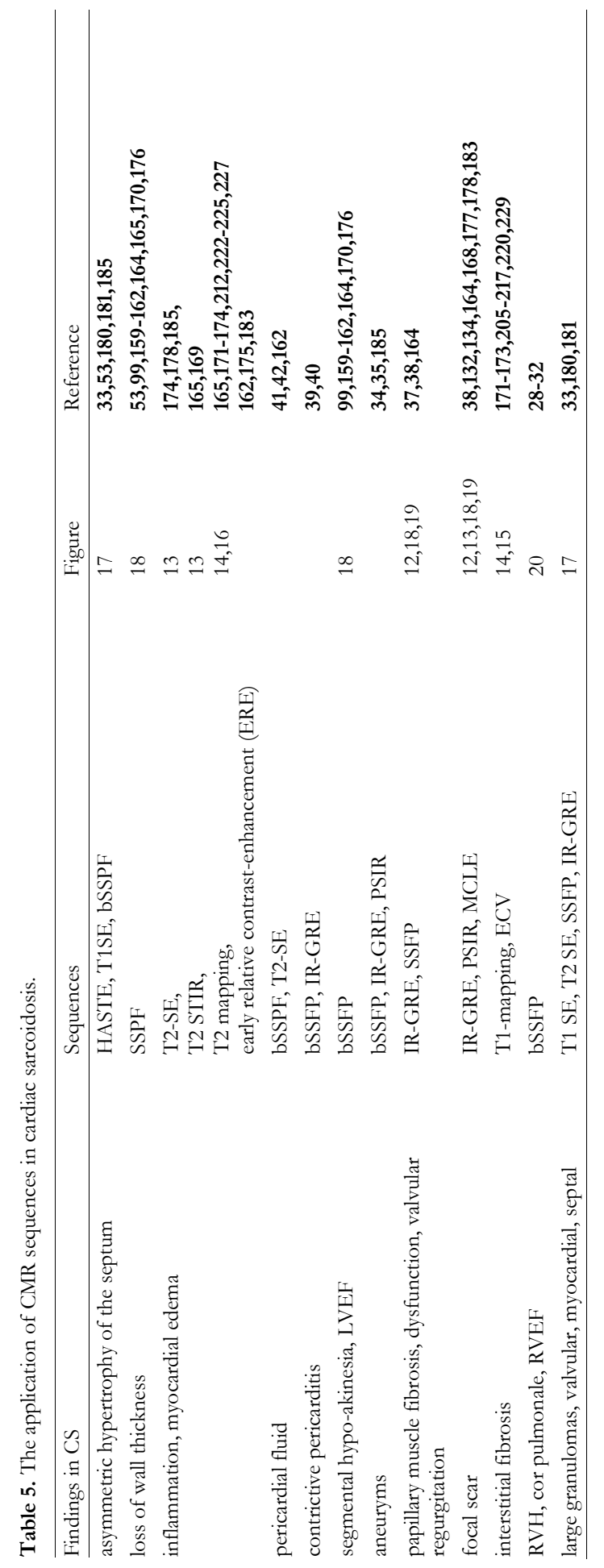




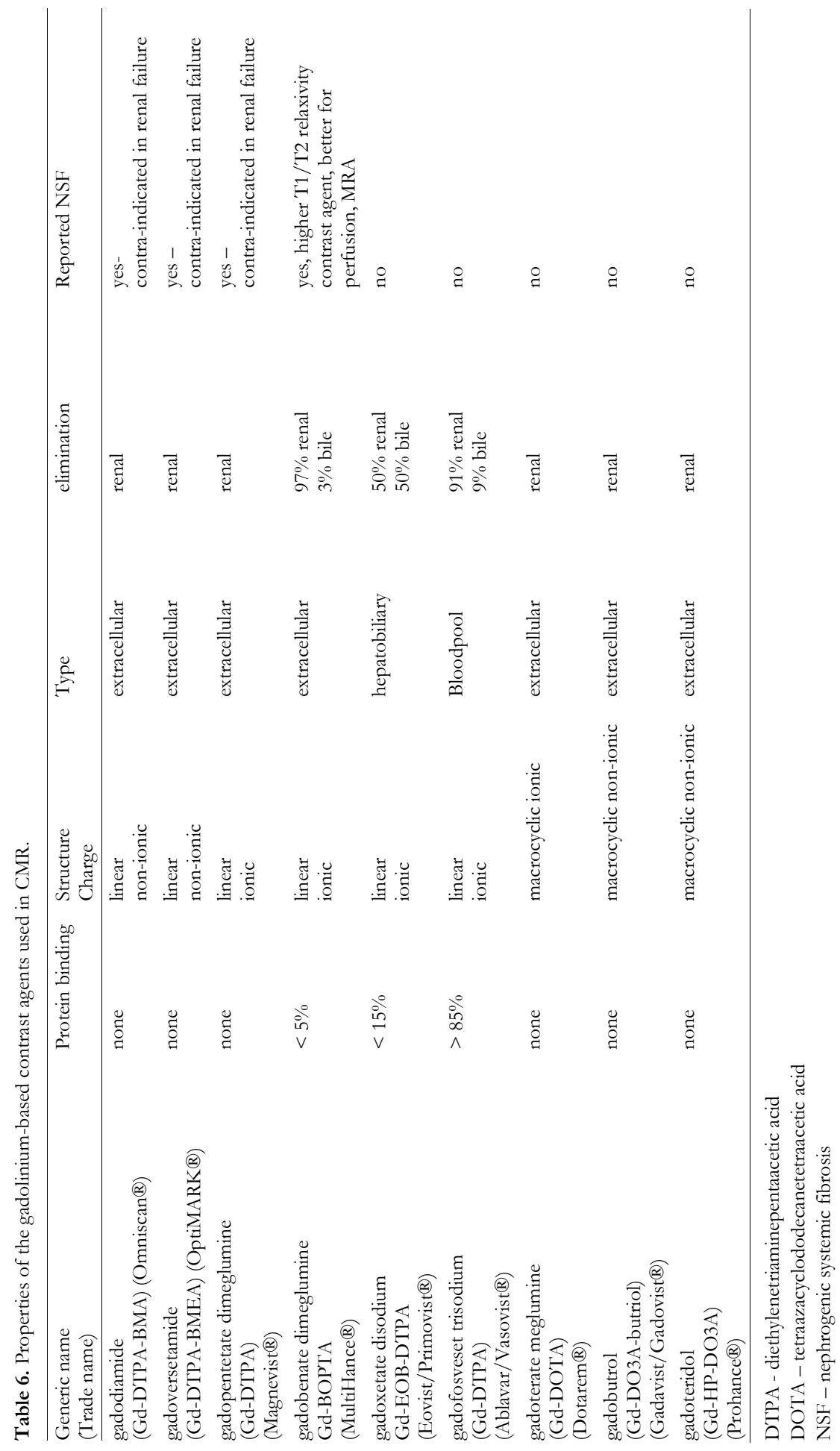


Chapter 2

Table 7. CMR - differential imaging diagnosis of cardiac sarcoidosis.

\begin{tabular}{|c|c|}
\hline Diagnosis & DECMR findings \\
\hline Coronary Artery Disease & $\begin{array}{l}\text { segmental edema/LGE subendocardial to transmural, coronary artery distribution, } \\
\text { matched segmental LV/RV systolic dysfunction, impaired EF (Figure 20,23) }\end{array}$ \\
\hline Viral Myocarditis & $\begin{array}{l}\text { predominant segmental LV edema/LGE, epicardial to transmural, particularly lateral } \\
\text { LV segments (Figure 23) }\end{array}$ \\
\hline Giant Cell Myocarditis & predominant LV edema/LGE, with wall motion abnormalities, impaired LVEF \\
\hline ARVC & $\begin{array}{l}\text { predominant segmental or global RV dilation/systolic dysfunction, segmental RV } \\
\text { dyskinesia, predominant RV LGE, segmental fatty RV infiltration/replacement } \\
\text { (Figure 25) }\end{array}$ \\
\hline Dilated Cardiomyopathy & $\begin{array}{l}\text { globular, dilated LV/RV, global/multi-segment systolic dysfunction } \\
\text { myocardial mid-layer LGE (Figure 23) }\end{array}$ \\
\hline Amyloidosis & $\begin{array}{l}\text { LVH, eventual dilation with systolic dysfunction, patchy, confluent or } \\
\text { subendocardial LGE (Figure 23) }\end{array}$ \\
\hline $\begin{array}{l}\text { Hypertrophic } \\
\text { Cardiomyopathy }\end{array}$ & SAM, segmental LVH with related focal LGE (Figures 22,23,26) \\
\hline Chagas & $\begin{array}{l}\text { segmental LV/RV systolic dysfunction - hypo-dyskinesia, LOWT, segmental } \\
\text { edema/LGE subendocardial to transmural, non-coronary artery distribution }\end{array}$ \\
\hline
\end{tabular}

LGE - late gadolinium enhancement, LOWT - loss-of-wall-thickness, LV- left ventricle, RV - right ventricle, SAM - systolic anterior motion of the anterior mitral valve leaflet 
Table 8. The role of CMR in the diagnostic Guidelines.

\begin{tabular}{|c|c|c|c|c|}
\hline Organization & Country & Year & Role of CMR & Reference \\
\hline $\begin{array}{l}\text { Japanese Ministery of Health } \\
\text { and Welfare }\end{array}$ & Japan & 1993 & None & 277 \\
\hline $\begin{array}{l}\text { Japanese Society of } \\
\text { Sarcoidosis and Other } \\
\text { Granulomatous Disorders }\end{array}$ & Japan & 2006 & $\begin{array}{l}\text { Diagnostic minor criterium - myocardial } \\
\text { LGE in a pattern compatible with CS } \\
\text { Diagnostic major criteria - basal thinning of } \\
\text { the interventricular septum }\end{array}$ & 250,266 \\
\hline $\begin{array}{l}\text { Japanese Society of Nuclear } \\
\text { Cardiology } \\
\text { Recommendations }\end{array}$ & Japan & 2014 & None, FDG guideline & 279 \\
\hline $\begin{array}{l}\text { New Japanese guidelines for } \\
\text { the diagnosis and treatment } \\
\text { of CS }\end{array}$ & Japan & 2017 & $\begin{array}{l}\text { Diagnostic major criterium - 1.myocardial } \\
\text { LGE in a pattern compatible with CS } \\
\text { 2.thinning of the basal IVS } \\
\text { 3.ventricular aneurysm } \\
\text { 4.LVEF }<50 \%\end{array}$ & 250 \\
\hline WASOG & International & 2014 & $\begin{array}{l}\text { Diagnostic: at least probable CS when LGE } \\
\text { in a pattern compatible with CS and/or } \\
\text { increased T2 signal on (DE)CMR indicative } \\
\text { of CS }\end{array}$ & \\
\hline $\begin{array}{l}\text { German Respiratory and } \\
\text { Cardiac Societies }\end{array}$ & Germany & 2014 & $\begin{array}{l}\text { Diagnostic: probable CS - in the presence } \\
\text { of diagnostic biopsies of extracardiac tissue, } \\
\text { CMR findings suggestive of CS (increased } \\
\text { T2 signal, wall motion abnormalities, } \\
\text { increased thickness, or loss of thickness, } \\
\text { LGE) } \\
\text { Diagnostic: certain CS - CMR findings of } \\
\text { CS in the presence of 12-lead resting ECG, } \\
\text { and/or Holter and/or TTE findings }\end{array}$ & 252 \\
\hline HRS & International & 2014 & $\begin{array}{l}\text { 1.Diagnostic: probable CS - LGE in a } \\
\text { pattern compatible with CS in pts with } \\
\text { biopsy proven extra-cardiac sarcoidosis } \\
\text { with either cardiac symptoms symptoms } \\
\text { and/or abnormalities on 12-lead resting } \\
\text { ECG, and/or TTE } \\
\text { 2.Risk stratification: particularly in pts with } \\
\text { LVEF > 35\%, diagnosis in patients with } \\
\text { extracardiac histology for sarcoidosis and } \\
\text { cardiac LGE in a pattern which suggests CS }\end{array}$ & 11 \\
\hline $\begin{array}{l}\text { European Association of } \\
\text { Nuclear Medicine, the } \\
\text { European Association of } \\
\text { Cardiovascular Imaging, and } \\
\text { the American Society of } \\
\text { Nuclear Cardiology. }\end{array}$ & Europe, USA & 2017 & $\begin{array}{l}\text { 1.Diagnostic value in suspected CS - } \\
\text { symptomatic pts with biopsy proven } \\
\text { extracardiac sarcoid and/or abnormal ECG } \\
\text { and/or inconcl cardiac US - LGE/T2 } \\
\text { 2.Suspected relapse of CS } \\
\text { 3.Prognostic value - LGE }\end{array}$ & 46,275 \\
\hline
\end{tabular}




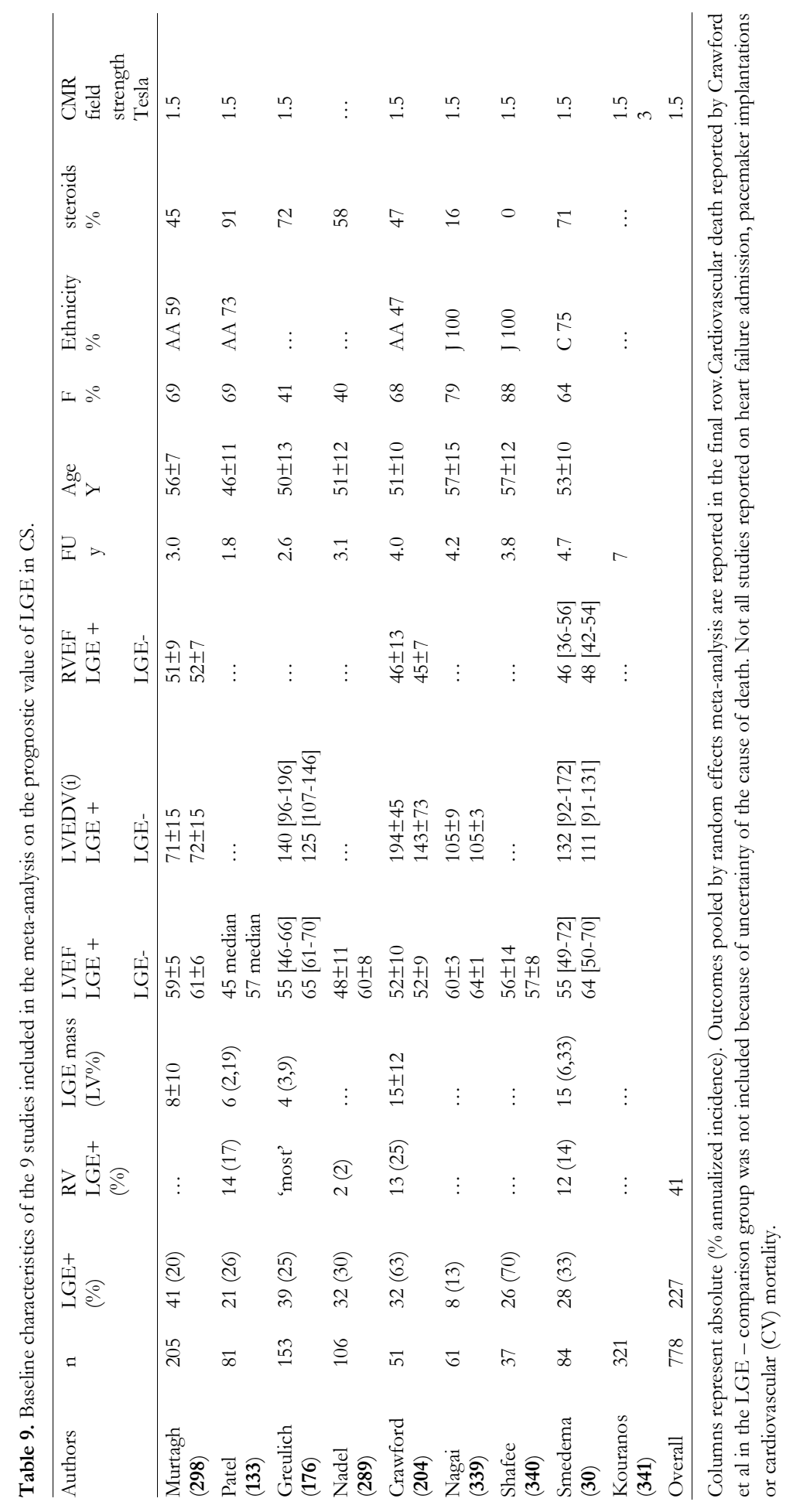




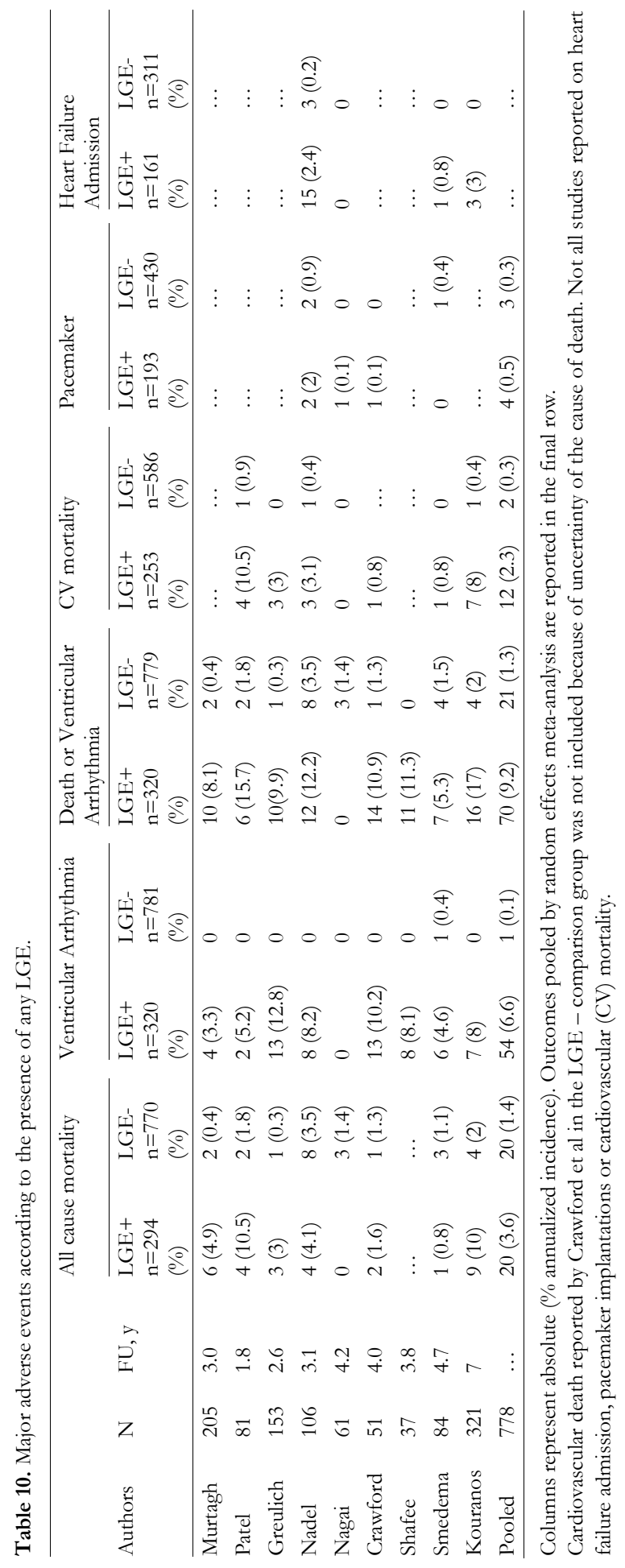




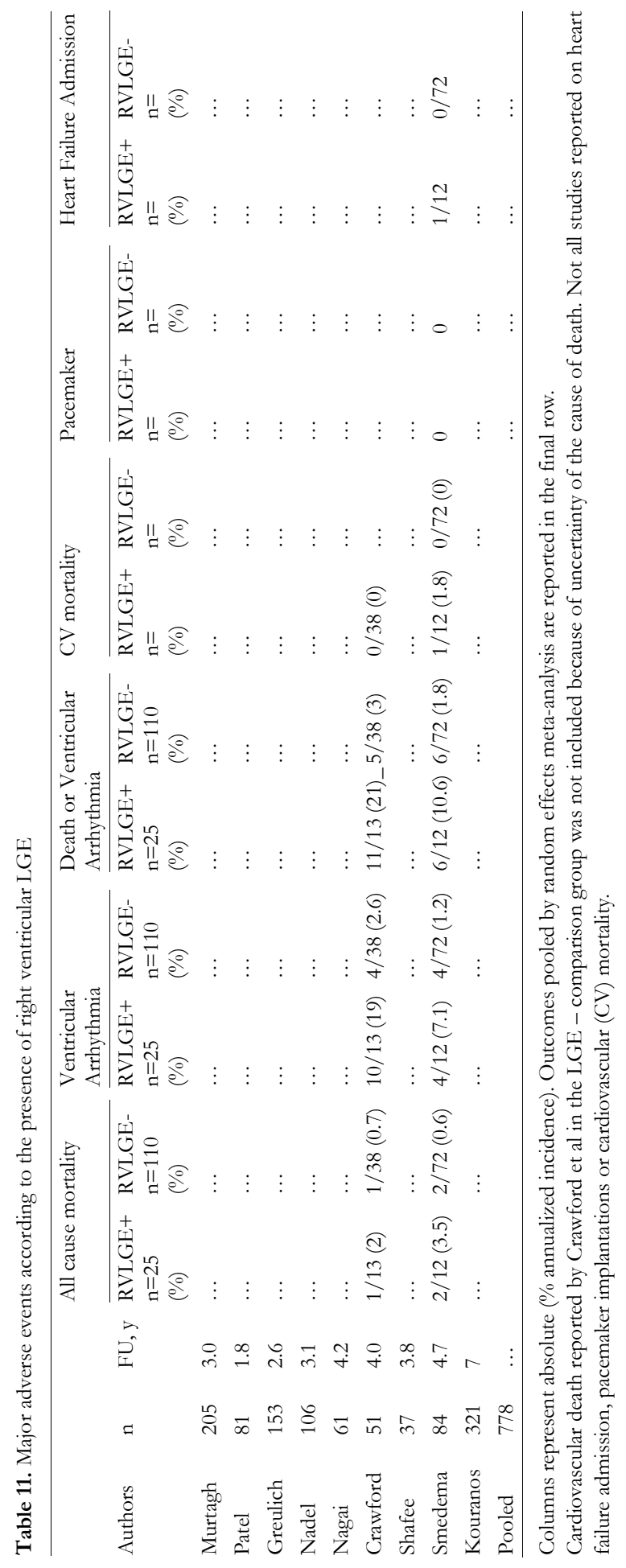




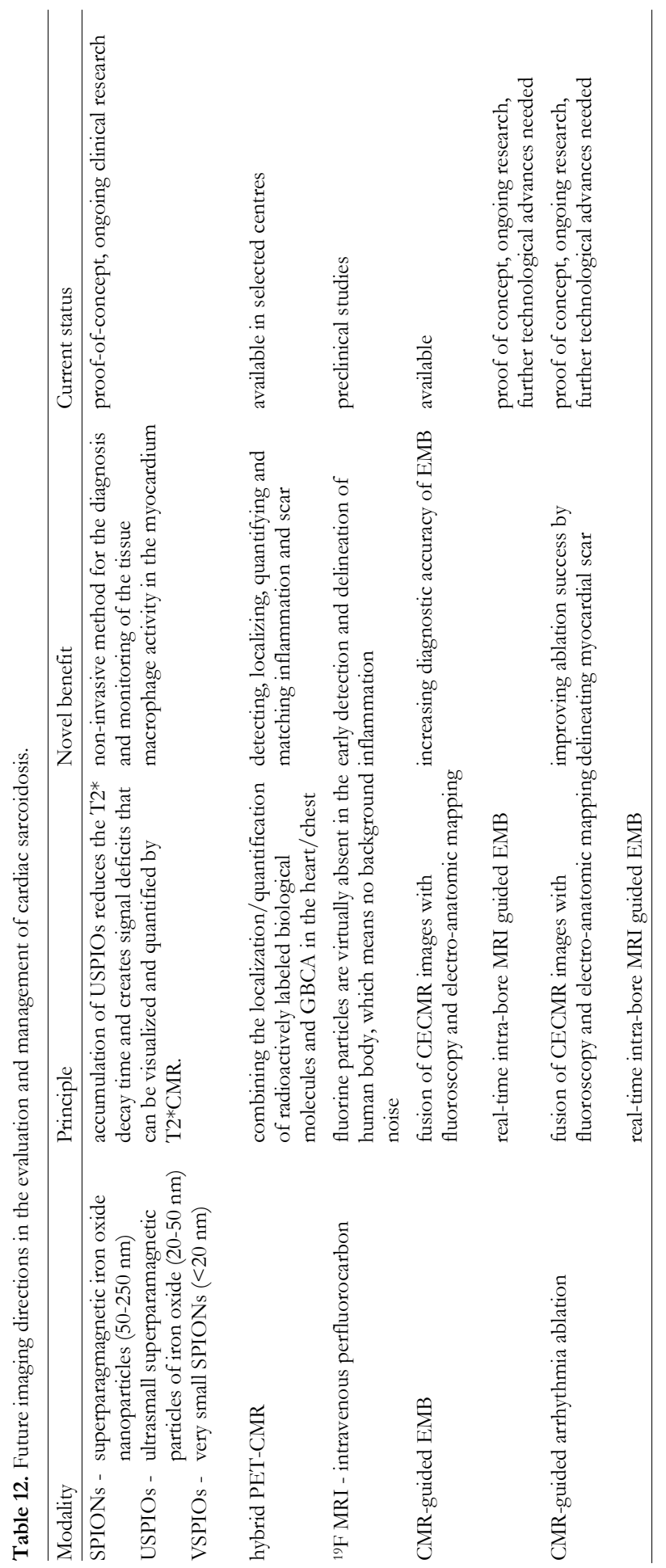




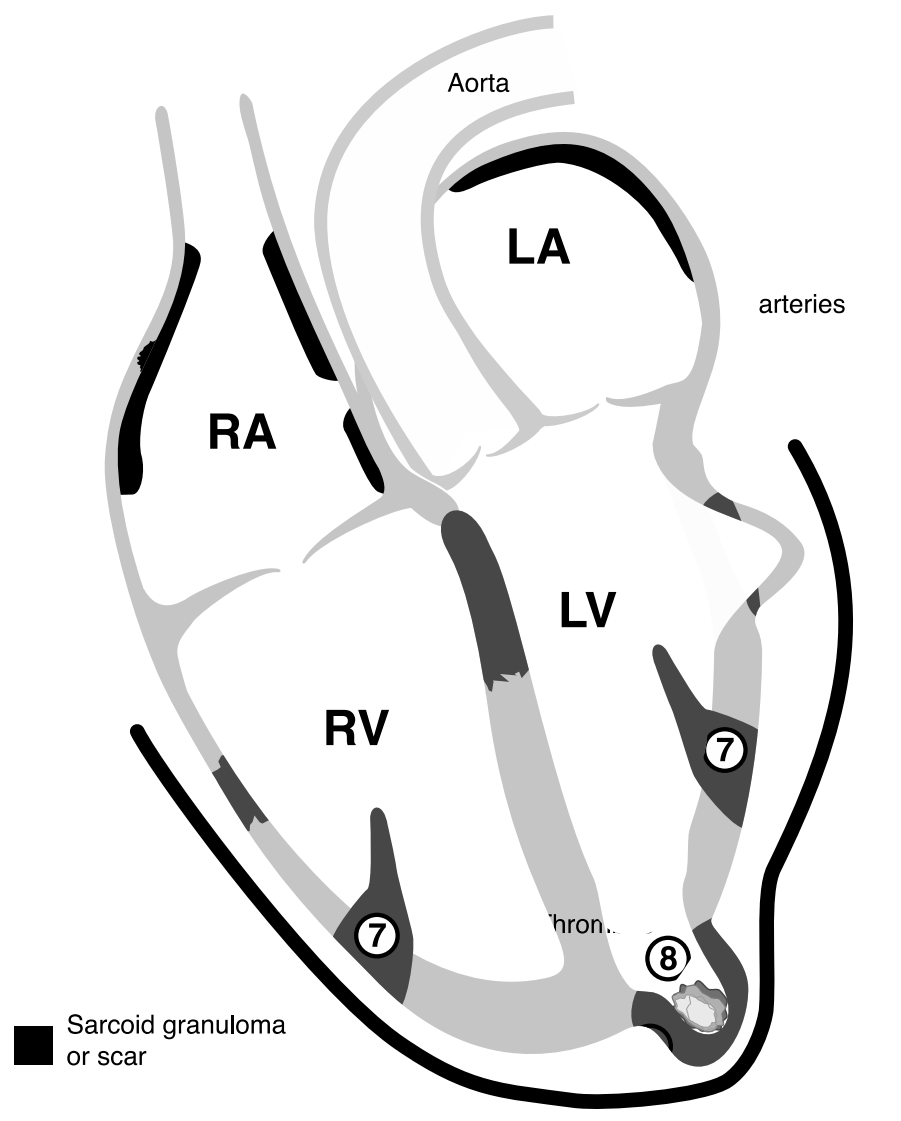

Figure 1. Distribution of cardiac involvement in sarcoidosis

1.Atrial infiltration and fibrosis resulting in atrial arrhythmias (AF, AFl, AT) (prevalence: frequent, post-mortem studies, case-reports) $(\mathbf{1 5 , 2 3 )}$

2.Coronary vasculitis (prevalence: rare, case-reports) $(24,25)$

3.Vasculitis of the aorta or pulmonary arteries $(9,15,26)$

4.Right ventricular infiltration and fibrosis (frequent, post-mortem series, case-reports) $(27,28,29,30,31)$

5.Granulomatous infiltration with asymmetrical hypertrophy and eventual fibrosis with loss of thickness of (the base of) the interventricular septum, resulting in atrio-ventricular conduction block (frequent, case-reports, postmortem series) $(\mathbf{1 5 , 1 6 , 3 2 )}$

6.Cardiac aneurysm, associated with corticosteroid use (rare, case-reports)

$(15,33,34)$

7.Granulomatous infiltration, fibrosis and secondary retraction of the papillary muscle(s) with tricuspid and/or mitral regurgitation (case-reports)

$(35,36,37)$

8.Intraventricular thrombus, secondary to myocardial fibrosis, dysfunction and aneurysm formation (15)

9.Pericardial granulomatous infiltration, pericardial effusion, and rarely tamponade or constrictive pericarditis $(38,39,40)$ 


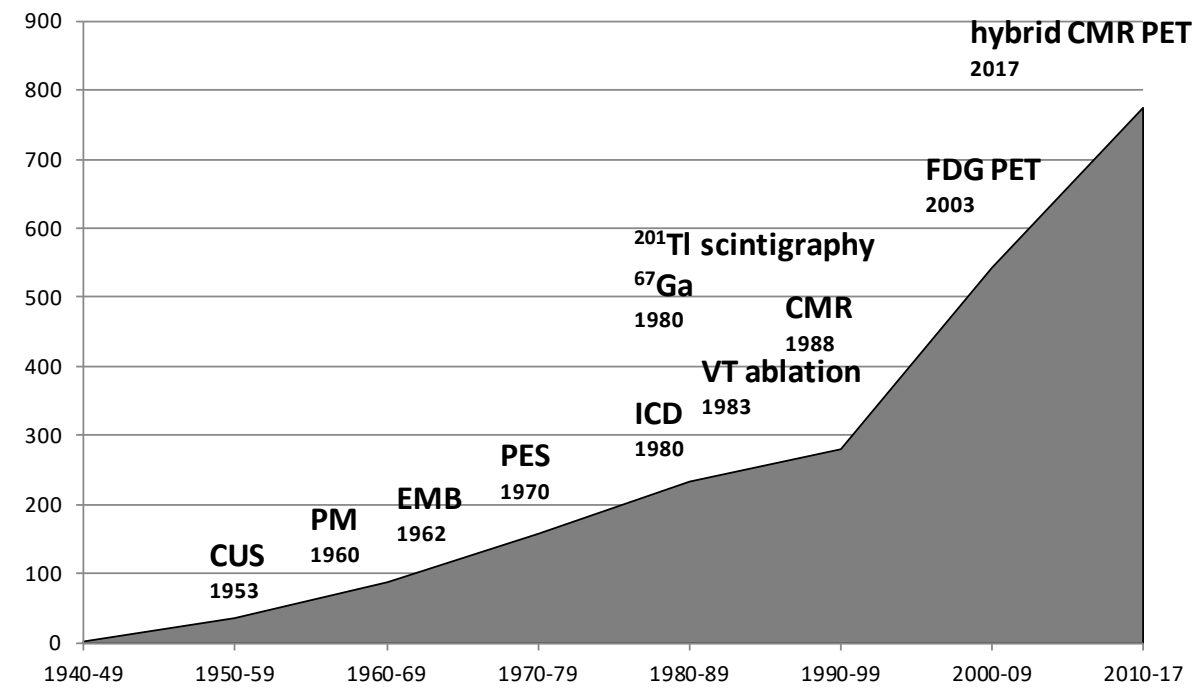

Figure 2. Number of publications on cardiac sarcoidosis included in Pub Med (1940-June 2017) related to the introduction of diagnostic methods and therapeutic interventions.

CUS-cardiac ultrasound, PM-pacemaker, EMB-endomyocardial biopsy, PES-programmed electrical stimulation; ICD-implantable cardioverter defibrillator, VT-ventricular tachycardia, CMR-cardiovascular magnetic resonance, $\mathrm{Tl}$ - thallium, Ga-gallium, FDG PET-fluorodeoxyglucose positron emission tomography,

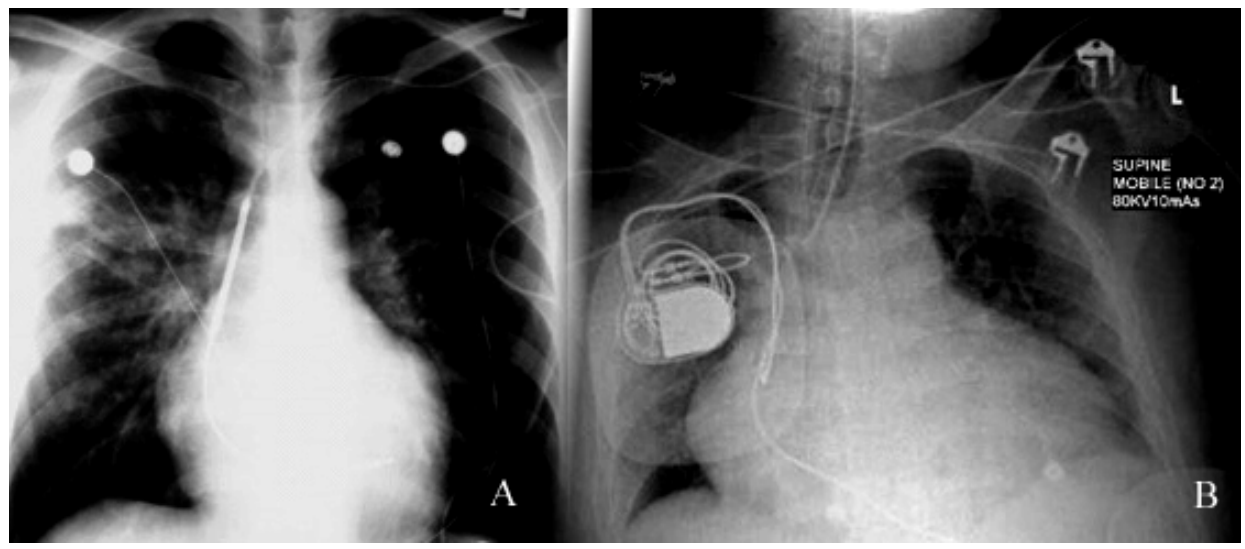

Figure 3. X chest (antero-posterior views) demonstrates mild cardiomegaly, bilateral reticulonodular changes predominantly affecting the upper lobes (stage 2 pulmonary sarcoidosis), and the presence of a single chamber implantable cardioverter defibrillator in a patient with cardiac sarcoidosis (left panel). The right-sided panel shows marked globular cardiomegaly in a patient with sarcoid cardiomyopathy, with a dual chamber pacemaker for third degree AVB in situ. He presented in cardiogenic shock with recurrent sustained VT (see 12 lead electrocardiogram below), was intubated (endotracheal tube in situ), ventilated and warranted recurrent electrical cardioversion (patches visible). 


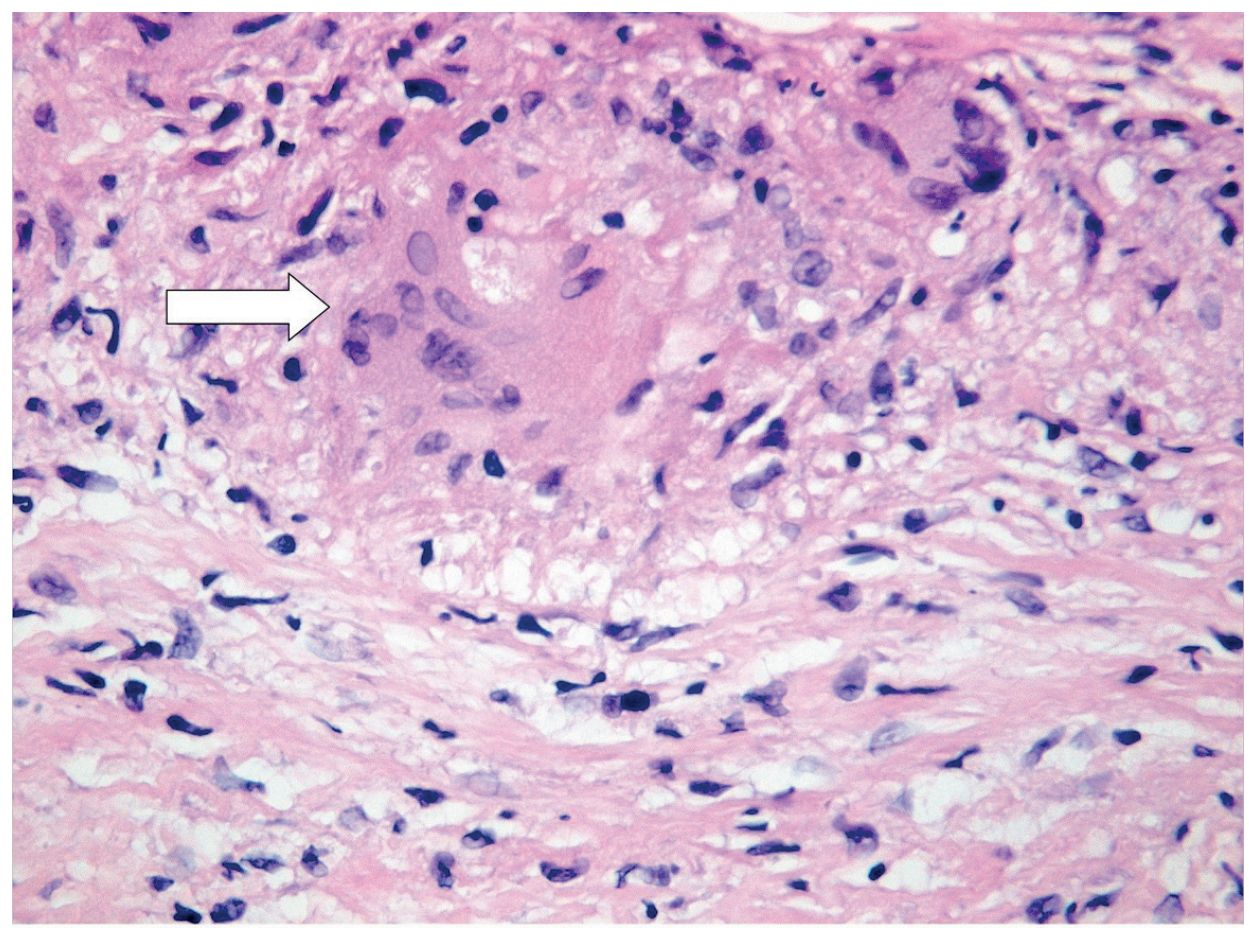

Figure 4. Endomyocardial biopsy demonstrates a sarcoid granuloma (arrow) consisting of giant cells, macrophages, CD4 lymphocytes, and hyaline sclerosis. 


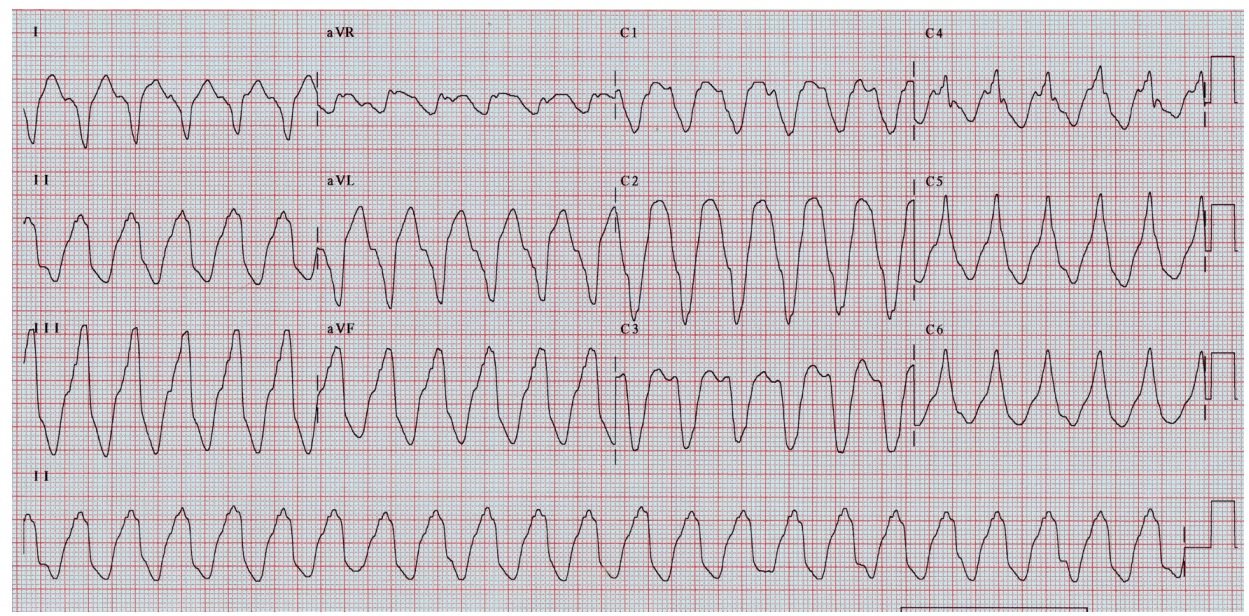

Figure 5. 12-lead electrocardiogram which demonstrates sustained monomorphic ventricular tachycardia (sVT).

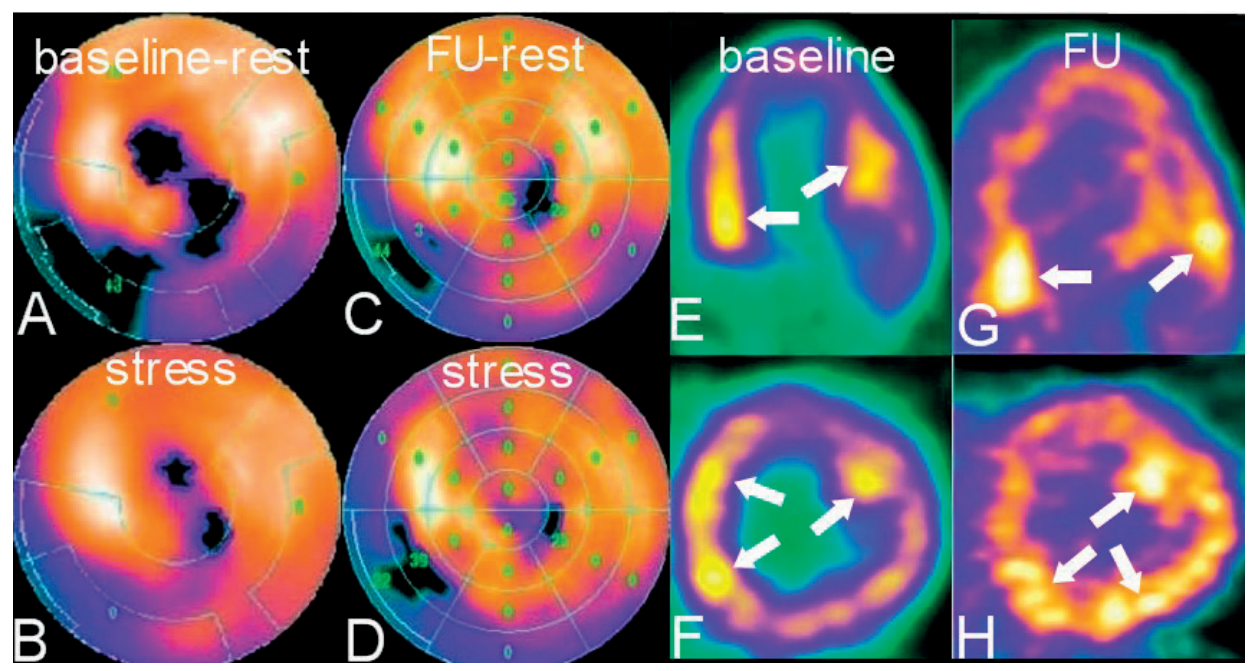

Figure 6A. A 63 year-old man presented with recent onset symptoms secondary to third-degree atrio-ventricular block, for which a dual chamber pacemaker was inserted. MIBI-technetium SPECT demonstrated "reversed uptake" in the septal and apical segments (bulls eye representation: A- rest image: apical and inferoseptal perfusion defects, B- stress image: apical perfusion defect decreased in size), suggestive of active granulomatous infiltration of these segments. FDG-PET showed increased nodular activity of the septum and anterolateral papillary muscle (E- horizontal long axis view, F- short axis view, equatorial slice; arrows point towards increased uptake). We diagnosed cardiac involvement according to the criteria published by the Japanese Ministry of Health (1996), and initiated treatment with oral steroids and methotrexate. Three months later his atrioventricular conduction had nearly recovered, with the percentage pacing being one. Follow up with MIBI-technetium SPECT revealed significant decrease of abnormalities, with small irreversible inferoseptal and apical defects remaining (C- rest image, Dstress image: small apical and inferoseptal perfusion defects). FDG-PET however still revealed increased nodular uptake in the inferior interventricular septum and papillary muscle (G- horizontal long axis view, $\mathrm{H}-$ short axis view, equatorial slice; arrows point towards increased uptake). Systolic ventricular function remained normal, without mitral valve regurgitation. 

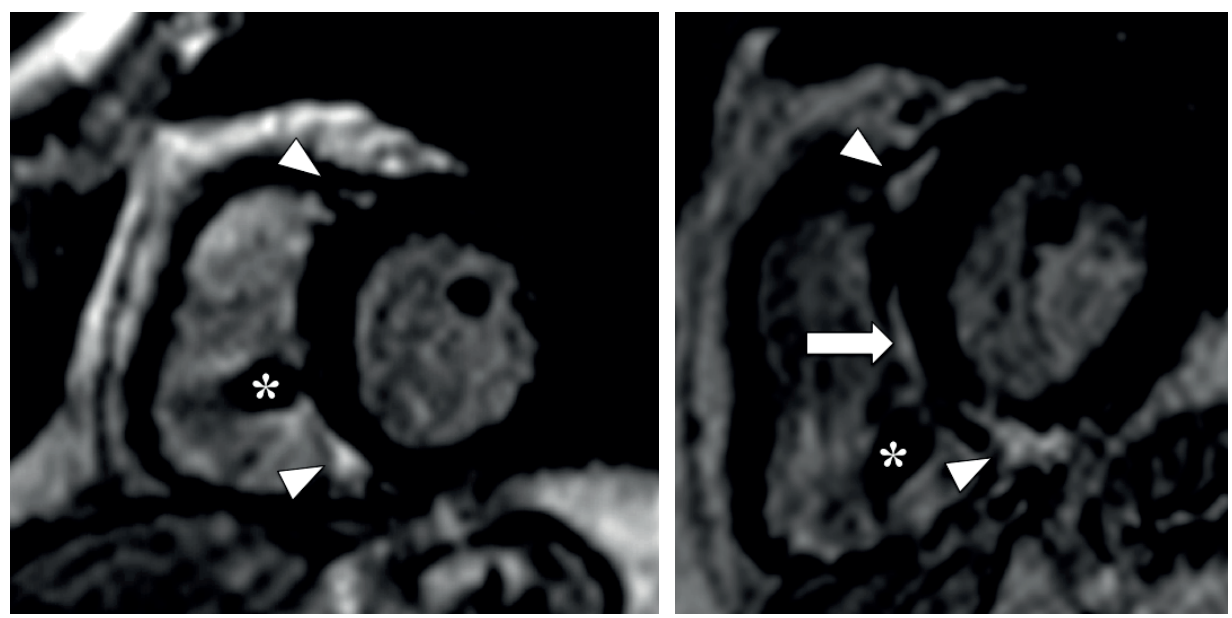

Figure 6B. DECMR study (Inversion-Recovery Gradient Echo sequence, end-diastolic frame, short axis view) in the same patient 3 months after the dual chamber pacemaker had been inserted. Ventricular insertion point enhancement is demonstrated (triangles). Pulmonary pressures were normal. (asteriks - artifact of right ventricular pace lead B: Seven years later DECMR demonstrates substantially more enhancement of the right-sided septum (arrow) and the insertion points (triangles). The percentage time pacing had increased from 5\% to $15 \%$ of the time. (asteriks $=$ artifact produced by right ventricular pace lead).

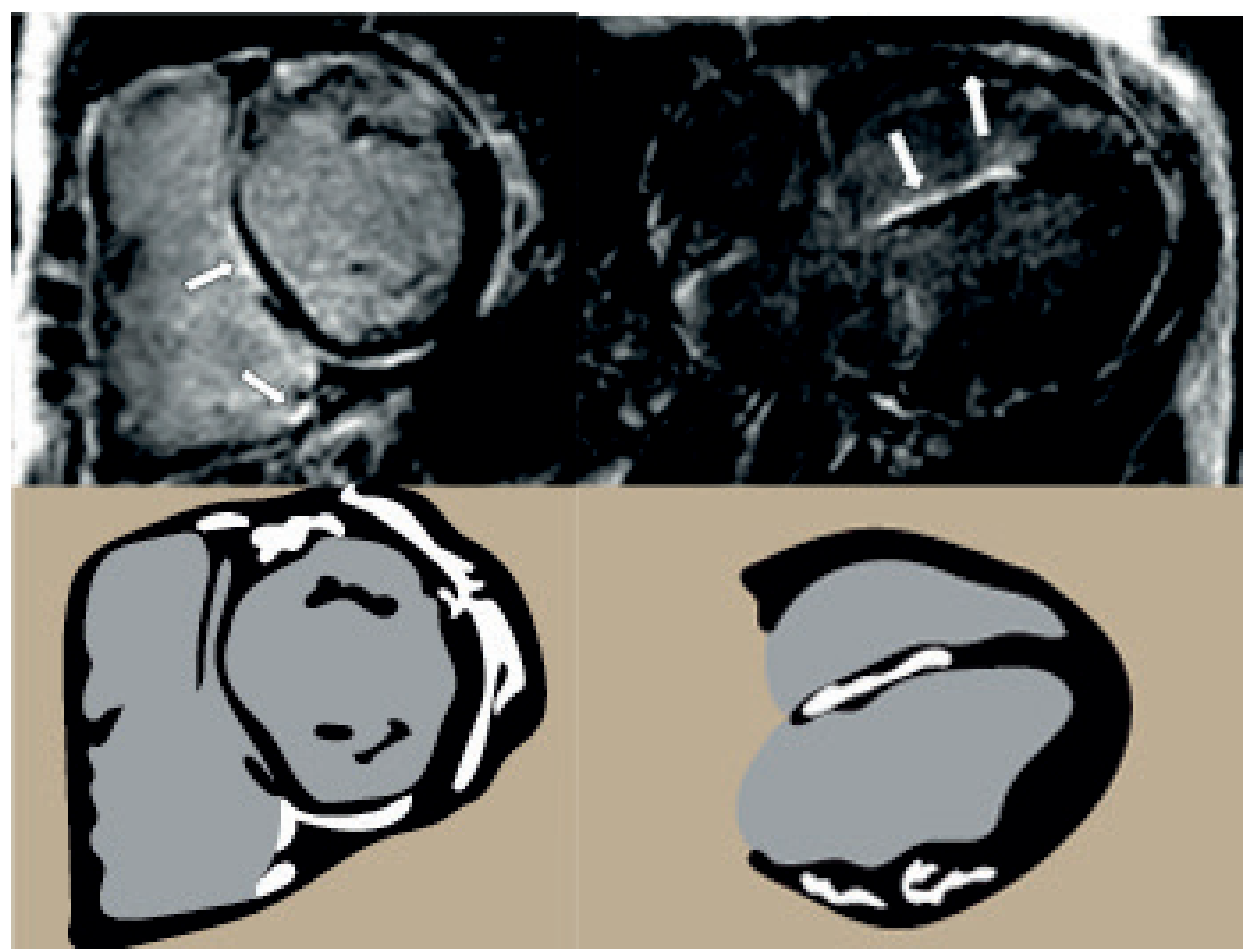

Figure 7A. Contrast-enhanced magnetic resonance study in a patient with biventricular congestive heart failure (left-sided panel, Inversion-Recovery Gradient Echo sequence, short axis view) demonstrates enhancement of the 
right-sided interventricular septum and inferior right ventricular insertion point. The horizontal long axis view of the identical patient demonstrates enhancement of the RV free wall and right-sided interventricular septum. (rightsided panel, Inversion-Recovery Gradient Echo sequence)
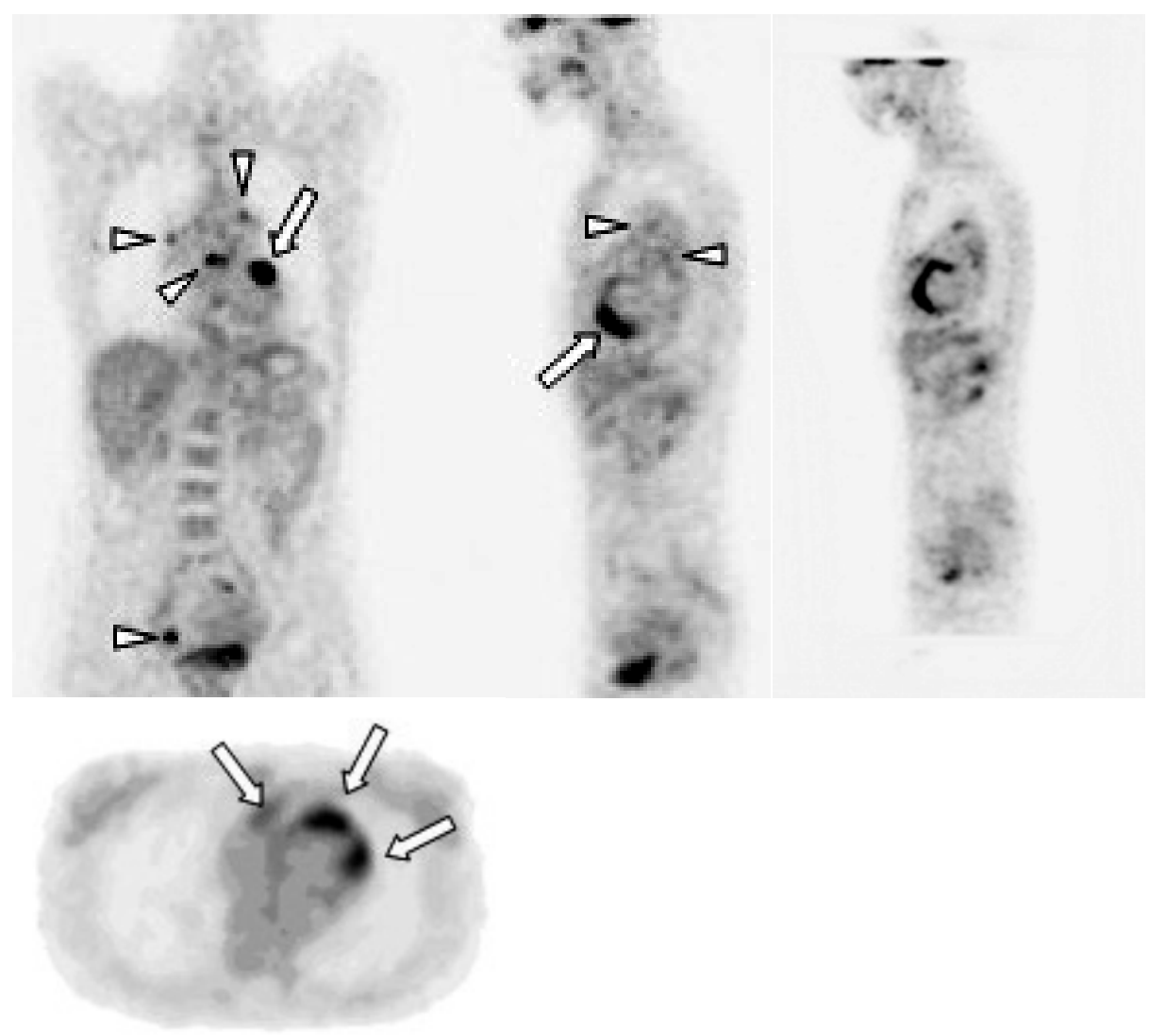

Figure 7B. FDG PET of this patient demonstrates sarcoid lymphadenopathy (triangles) and active inflammation of the antero-apical LV segments and RV free wall (arrows). 


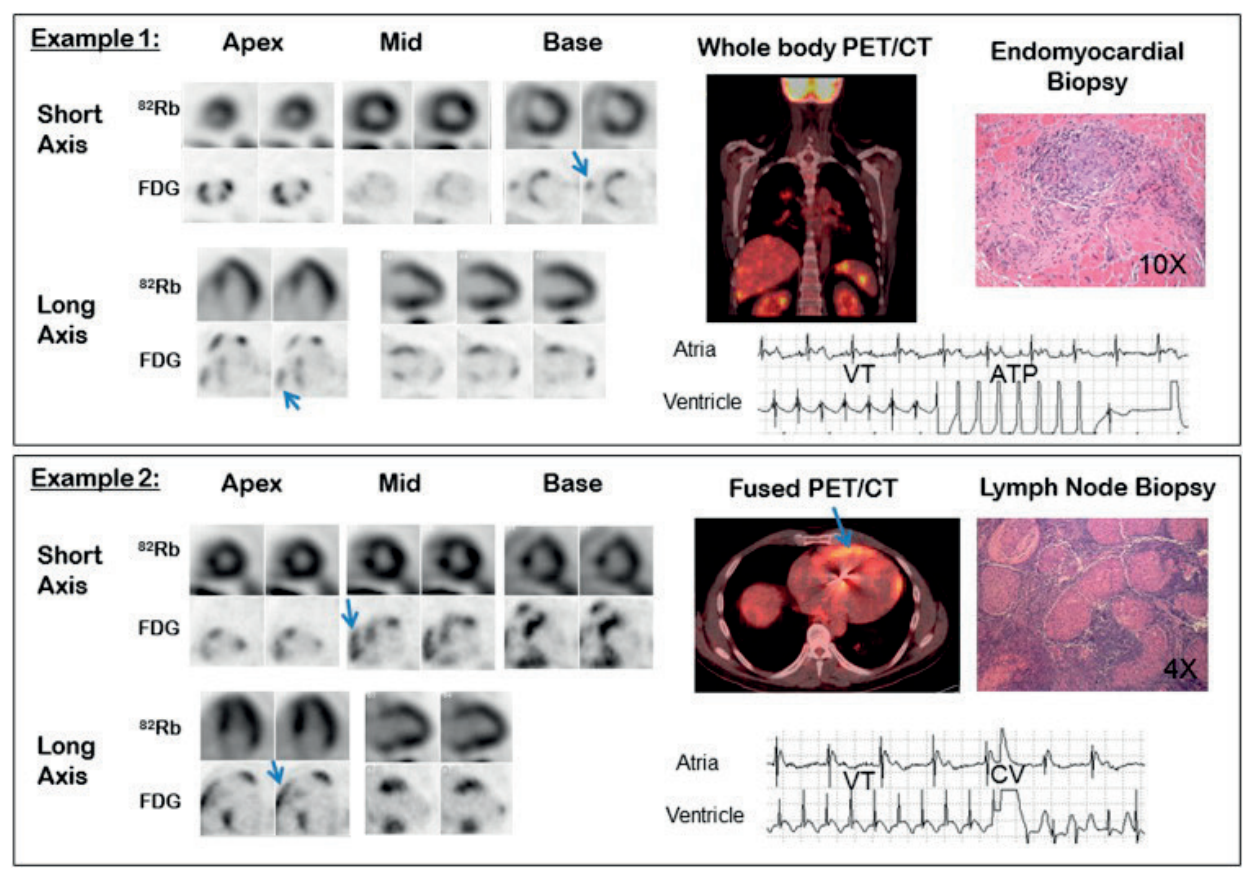

Figure 8. Examples of focal inflammation of the RV due to sarcoidosis. Example 1: A 50 year-old female had pacemaker implantation for pre-syncopal episodes because of high degree block due to sarcoidosis. PET/CT for suspected CS identified a medium sized perfusion defect throughout the basal septum with increased FDG uptake ("mismatch pattern"). There was also FDG uptake involving the apex and RV free wall (arrow). Whole-body images show FDG uptake in the liver and spleen and in the paratracheal lymph nodes. EMBx identified the presence of granulomas consistent with the diagnosis of sarcoidosis. Interrogation of her ICD 3 month later identified the presence of rapid VT at a rate of 200 beats/min, which required anti-tachicardia pacing therapy. Example 2: 48-year-old male with pulmonary sarcoidosis who was referred for PET/CT for suspected cardiac involvement. He was found to have a perfusion defect associated with focal FDG uptake along the basal anterior and inferior septum as well as multiple focal areas of FDG uptake thoughout the RV (arrows). Lymph node biopsy results confirmed the presence of sarcoidosis. Less than 1 month after ICD implantation he had an episode of VT (see rhythm strip). CT = computed tomography; PET = positron emission tomography. (Blankstein R, Osborne M, Naya M, Waller A, Kim CK, Murthy VL, Kazemia P, Kwong RY, Tokuda M, Skali H, Padera R, Hainer J, Stevenson WG, Dorbala S, Di Carli MF. Cardiac Positron Emission Tomography Enhances Prognostic Assessments of Patients With Suspected Cardiac Sarcoidosis. J Am Coll Cardiol 2014;63(4):329-336. Reproduced with permission from the publisher) 


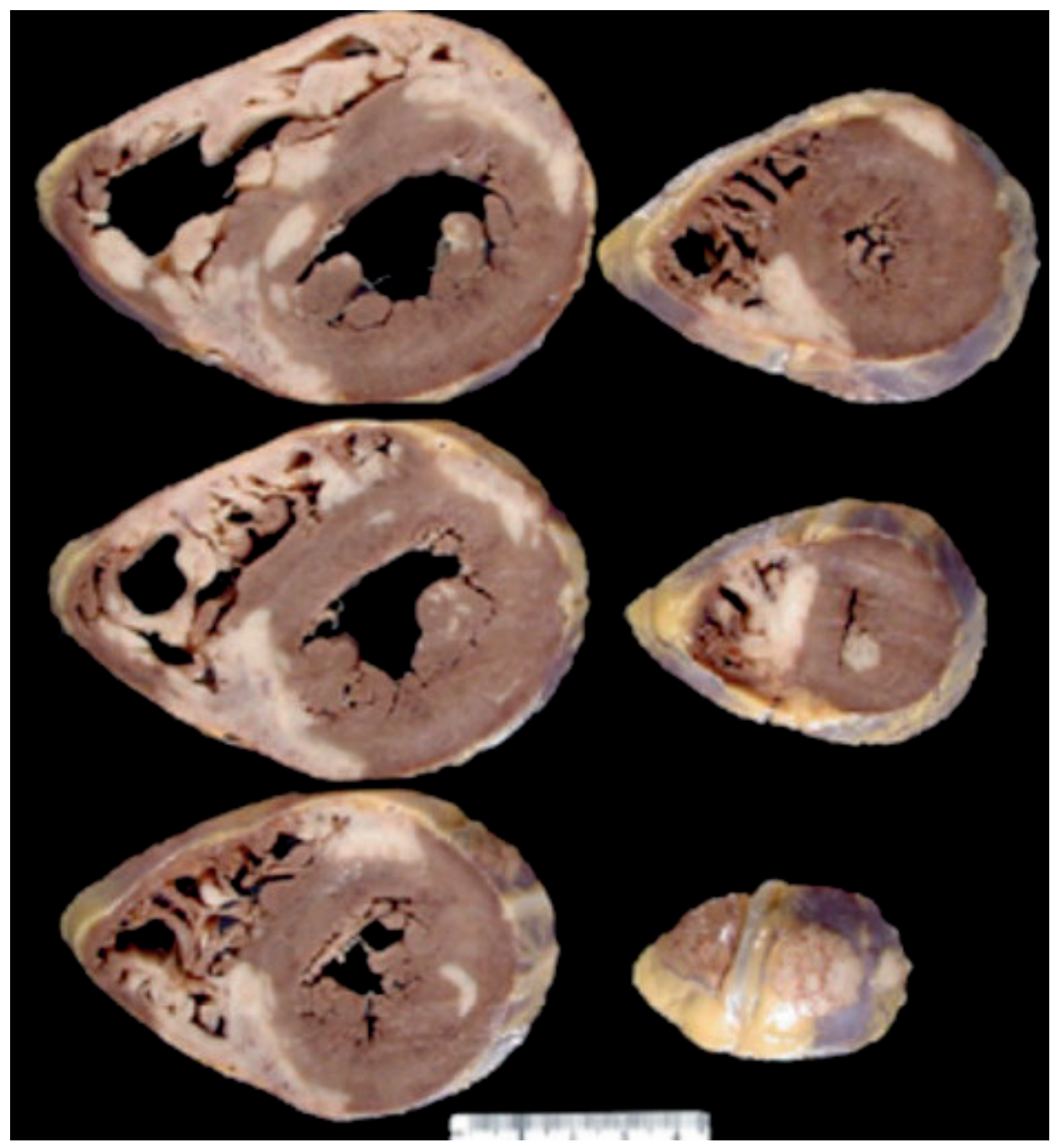

Figure 9. Pattern of sarcoid involvement, apex to base. Often haphazard involvement of myocardium is present with variation from apex to base. However, a propensity was seen in this heart for posterior septal involvement, anterior subepicardial involvement, and right ventricular involvement, which in this heart, was anterior towards the base, and posterior throughout, merging with the septal disease. The apical tip demonstrates epicardial sarcoid. (Tavora F, Cresswell N, Li L, Ripple M, Solomon C, Burke A. Comparison of Necropsy Findings in Patients With Sarcoidosis Dying Suddenly from Cardiac Sarcoidosis Versus Dying Suddenly from Other Causes. Am J Cardiol 2009;104:571-577. Reproduced with permission from the publisher.) 


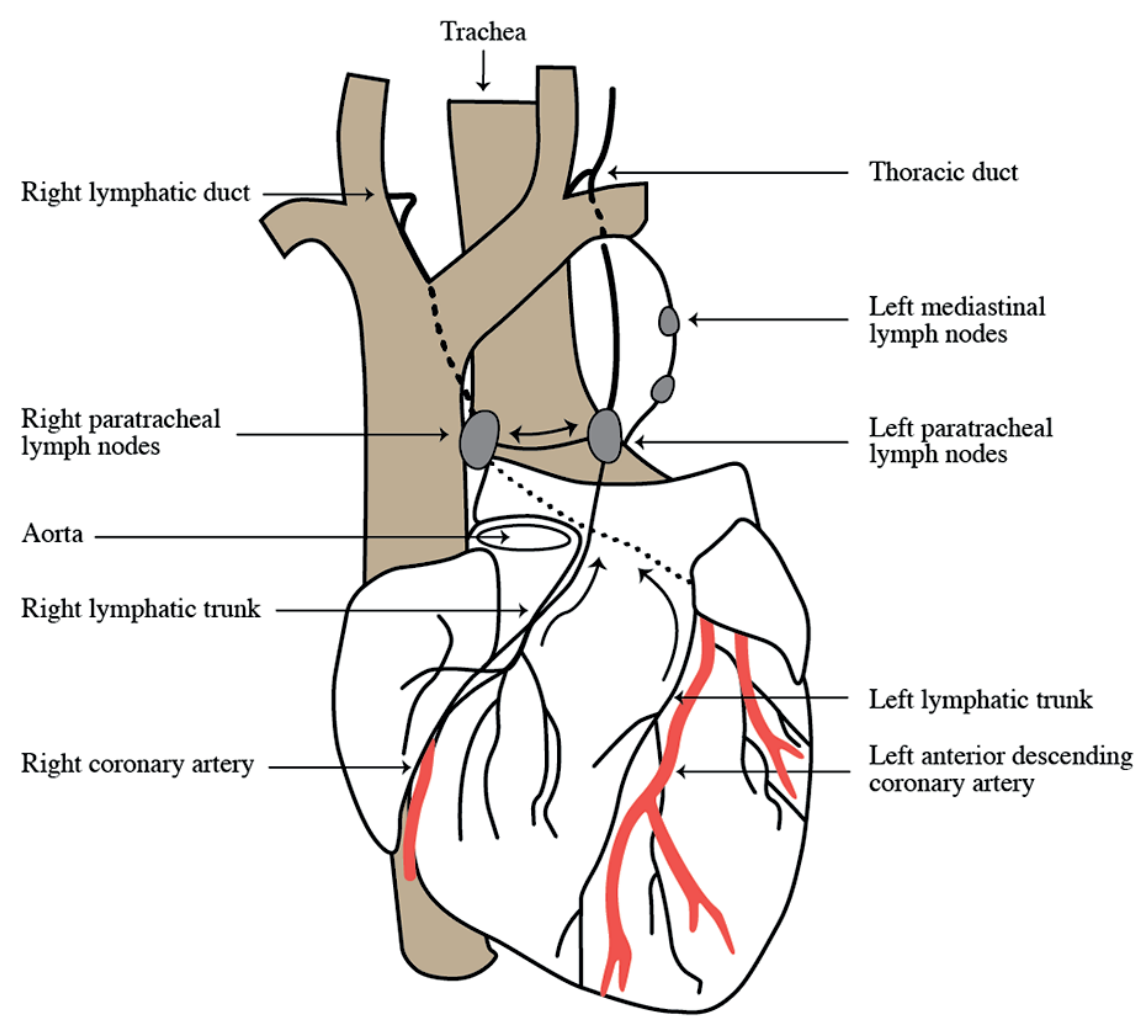

The right lymphatic trunk drains into the left paratracheal nodes and the left anterior mediastinal nodes, and the left lymphatic trunk drains into the right paratracheal nodes. The right and left paratracheal nodes are connected.

Figure 10: Distribution of the cardiac lymph vessels and regional lymphe nodes. 
A

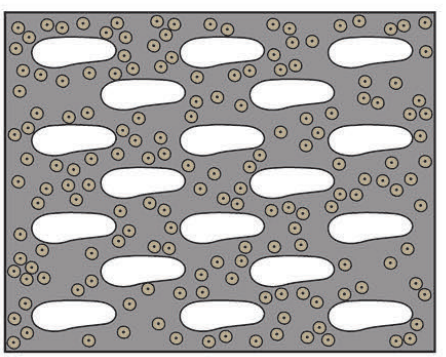

C

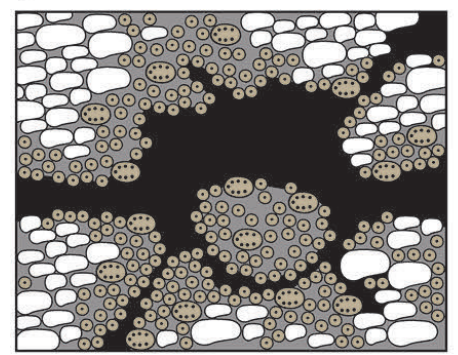

B

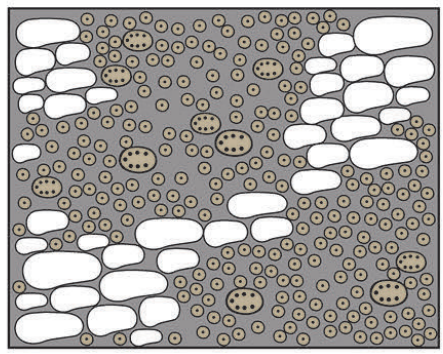

D

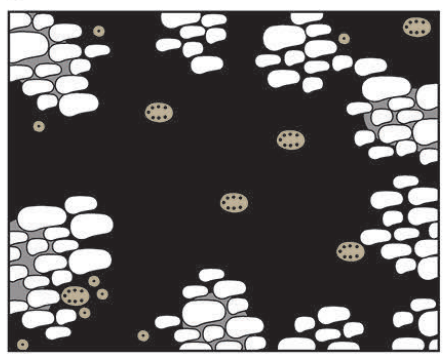

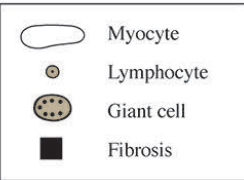

Figure 11. Schematic histopathology of cardiac sarcoidosis. (A. Stage I: early inflammation, lymphocytic infiltration, interstitial edema, B. Stage II: granuloma formation, epitheloid and giant cells, C. Stage III: infiltration of the myocardium by inflammatory cells with fibrosis, D. Stage IV: extensive replacement fibrosis, myocardium replaced by fibro-hyaline changes resulting in focal scar.
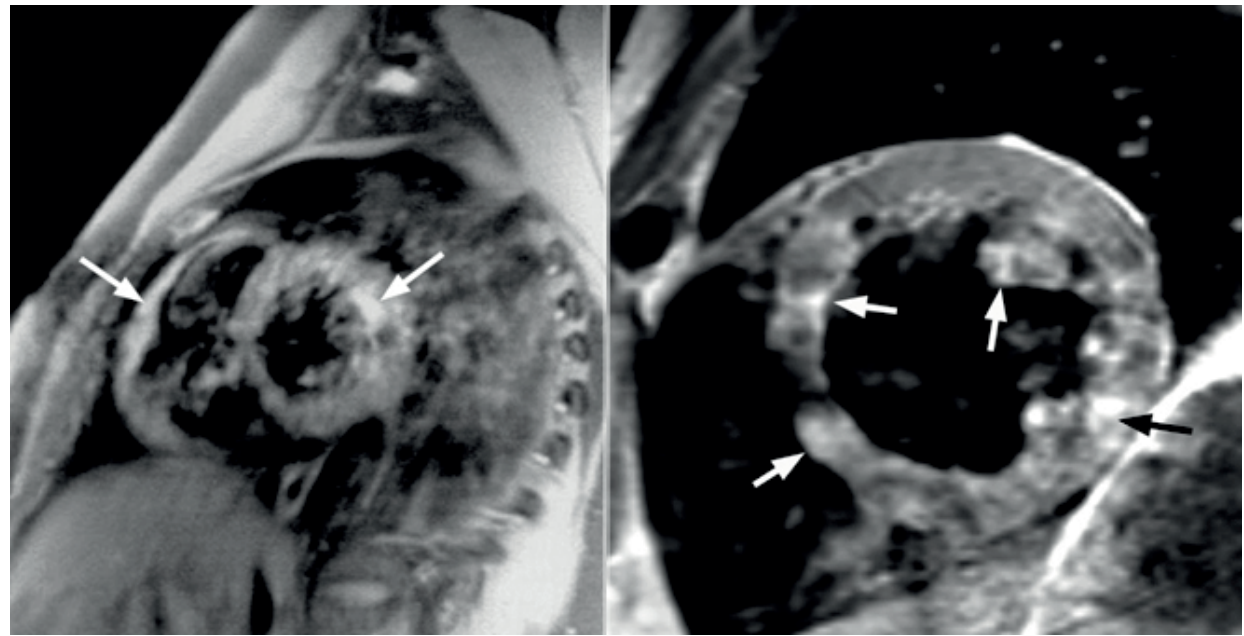

Figure 12. Delayed-enhanced T1-weigthed black-blood spin echo images (short axis views) demonstrate enhancement of the free RV wall (left panel), patchy three layer enhancement of the interventricular septum (right panel), and the antero-lateral papillary muscle and lateral left ventricular segments (left and righty panels).

$\square$ LGE $\square$ blood pool $\square$ unenhanced myocardium 


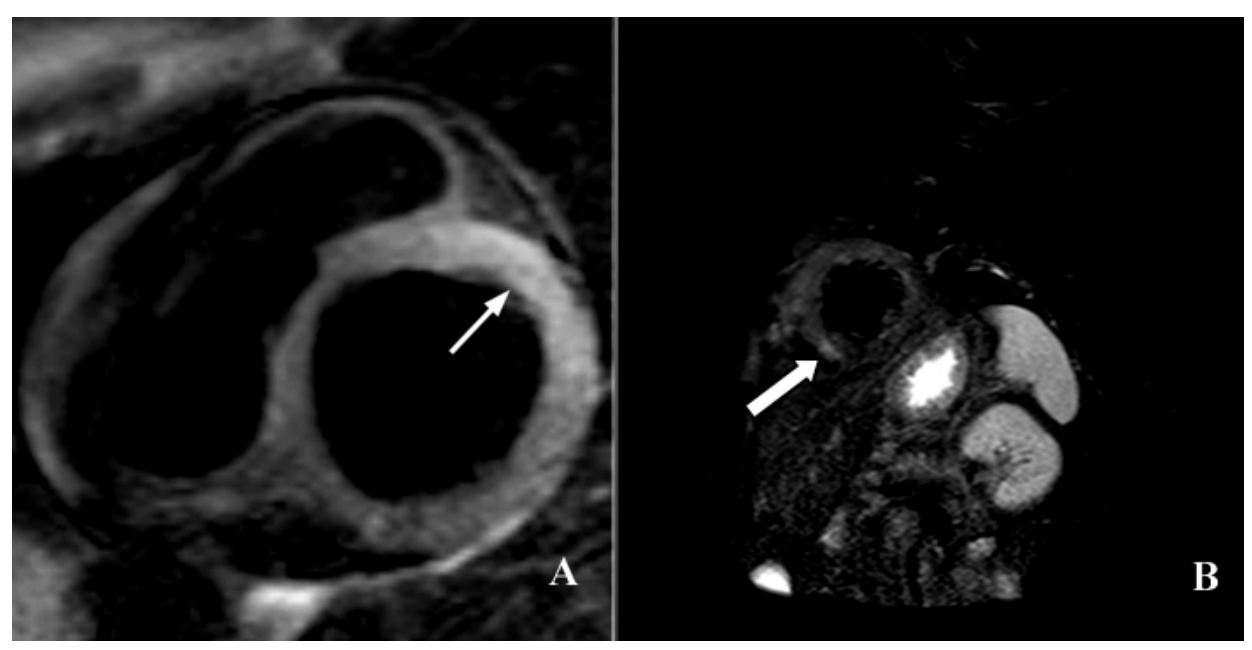

Figure 13. T2 SE and T2 SE STIR demonstrate active cardiac sarcoidosis. Panel A reveals diffuse transmural edema in the basal anterolateral LV, while panel B shows focal granulomatous infiltration in the interior interventricular septum.

$\square$ myocardial edema/granuloma $\square$ blood pool $\square$ unenhanced myocardium 


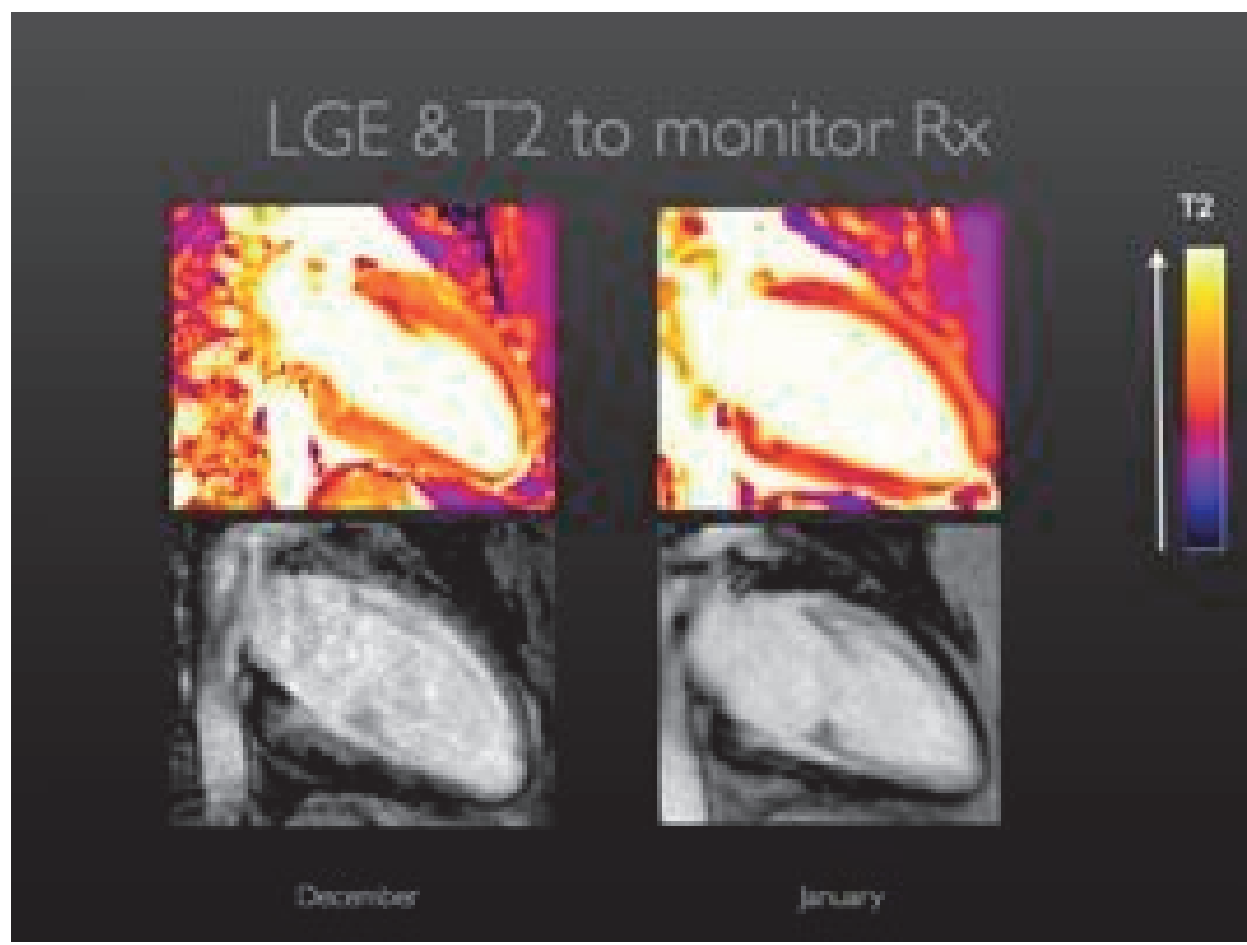

Figure 14. A 44 year-old male presented with non-specific systemic symptoms and dyspnea. T2 mapping shows prolonged T2 in the inferior and anterior segments (left upper panel). EMBs taken from the interventricular septum were diagnostic of CS. T2 The LGE in the interior and anterior segments signified active granulomatous infiltration and cardiomyocyte degradation (left lower panel). Follow up mapping shows recovery of prolonged T2 in the inferior and anterior segments after treatment with corticosteroids for active CS (right upper panel). The LGE in the interior and anterior has largely resolved. The remaining LGE suggest remaining focal scar (right lower panel). I kindly acknowledge support by dr Anna Herrey who managed this patient and provided the images. 

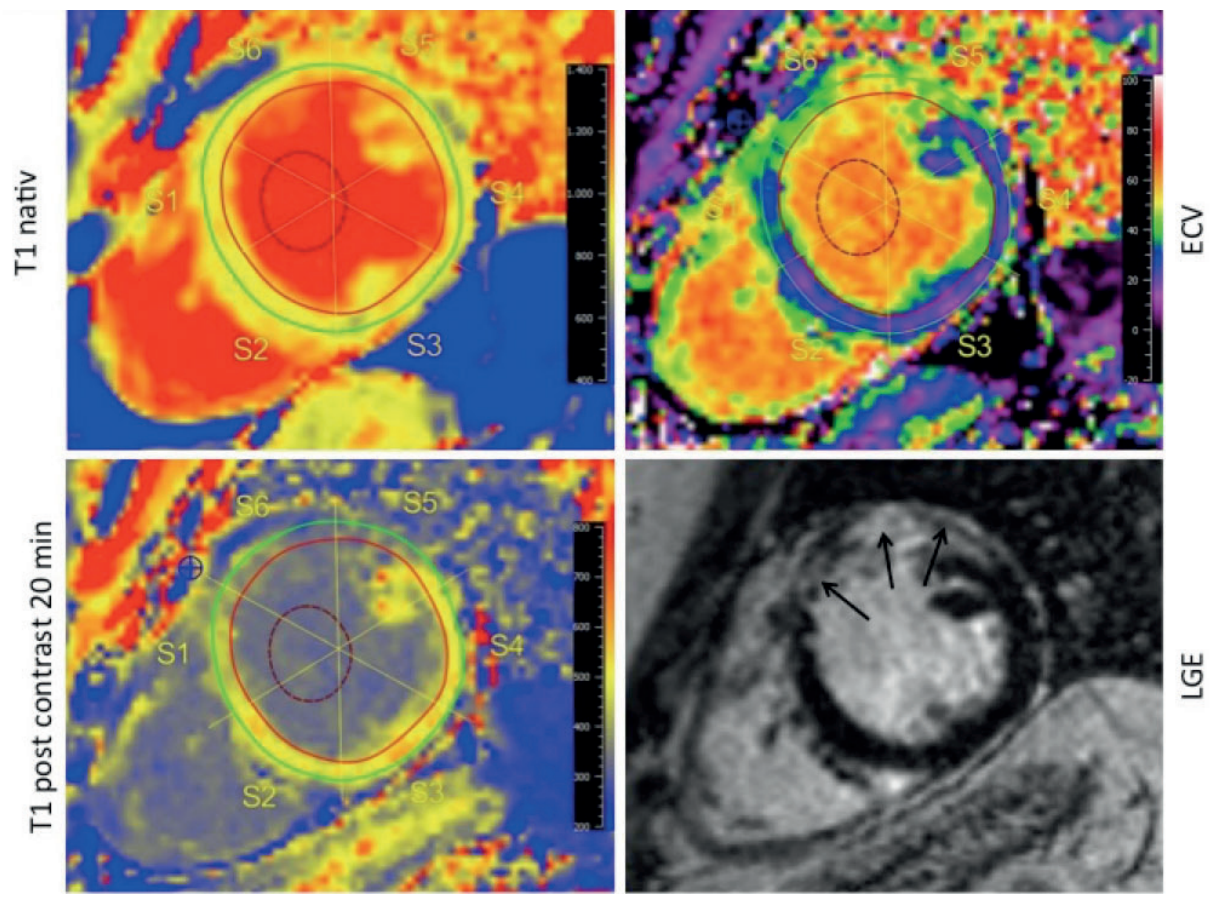

Figure 15. CMR of a 44-year-old female presenting with palpitations and RBBB. Cine images revealed a preserved LVEF (51\%) with hypokinesia in the anterior wall. LGE demonstrated a diffuse pattern of epicardial, intramural and transmural enhancement in the anterior wall, suggestive of CS. The native T1 map showed an increased T1 with $1040 \mathrm{~ms}$ (normal median 960 [942-986] ms), expanded ECV of 36\% (25 [23-27]\%), and decreased postcontrast T1 with $452 \mathrm{~ms}$ (526 [509 -552]ms). Coroconary artery disease was ruled out, and EMB confirmed CS. Reproduced with the permission of the publisher. 

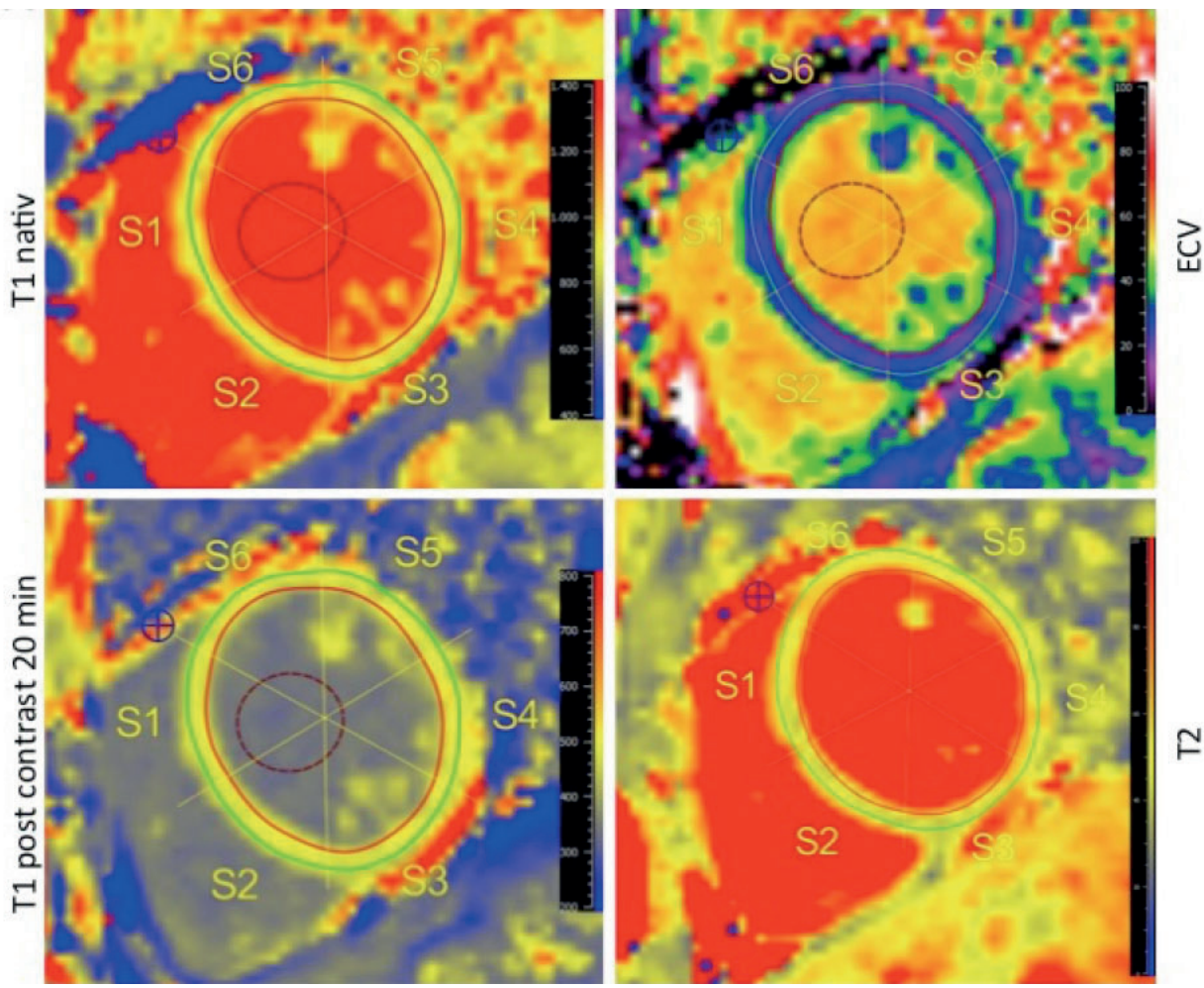

Figure 16. CMR of a 27 -year-old female with a history of pulmonary sarcoidosis since two months. She experienced recurrent chest pain. CMR revealed preserved LVEF with normal dimensions. DECMR did not demonstrate LGE. However, native T1 (1004 ms), ECV (26\%) and T2 (53 ms) were increased, and post contrast T1 (483 ms) decreased compared with our control group (median native T1 960 ms, ECV 25\%, postcontrast T1 526 ms, T2 49 ms). Reproduced with the permission of the publisher.
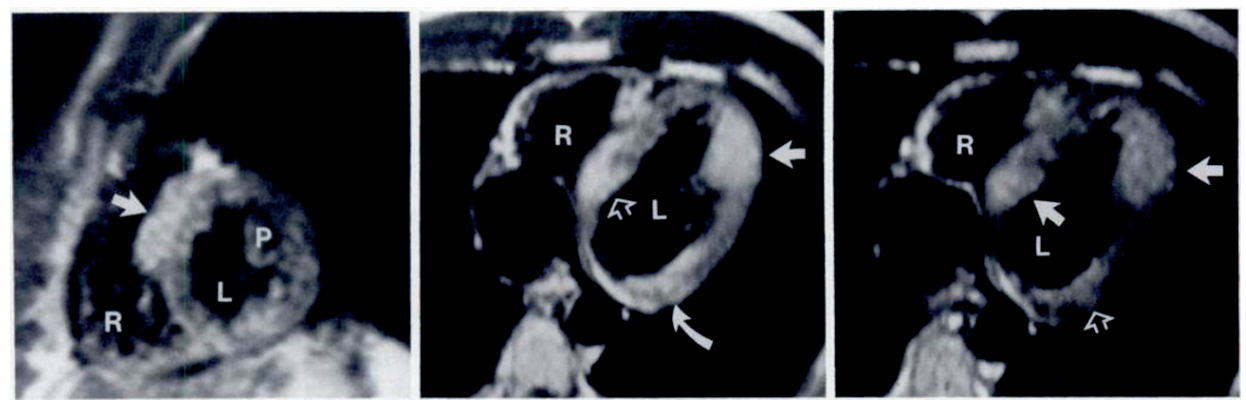

Figure 17. First ever published MR Images (0.5 T magnet, TR $750 \mathrm{msec}$, TE 10-100 msec, $12 \mathrm{~mm}$ slice thickness, matrix $256 \times 256$ ) of biopsy confirmed cardiac sarcoidosis, in a 30-year-old Caucasian male who presented with symptoms of a third degree atrio-ventricular block. (A: short axis image, $\mathbf{B}$ and $\mathbf{C}$ transverse images, arrows signify focal areas of granulomatous infiltration resulting in hypertrophy and increased signal intensity; $\mathrm{P}=$ posterolateral papillary muscle, $\mathrm{L}=$ left ventricle, $\mathrm{R}=$ right ventricle)

From Riedy K, Fisher MR, Belic N, Koeningberg DI. MR Imaging of myocardial sarcoidosis. AJR 1988;151:915916. Reproduced with the permission of the American Roentgenologic Society. 


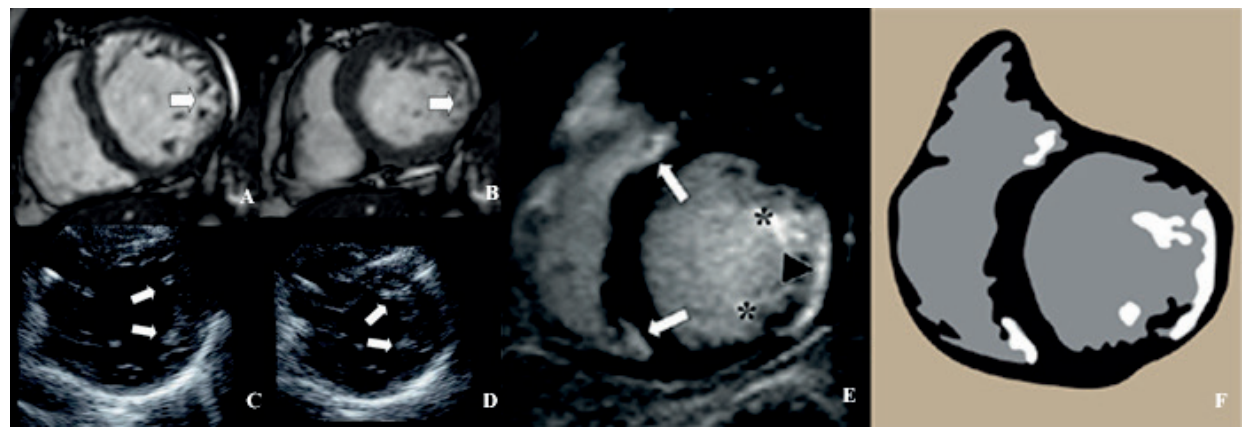

Figure 18: Cardiac ultrasound and DECMR study (SSFP and IR-GRE sequences, short axis views) in a patient without pulmonary hypertension demonstrate posterolateral loss-of-wall-thickness (LOWT) (A,B), hyperechoicity (C,D) and LGE of the ventricular insertion points (arrows), papillary muscles (asterisks) and postero-lateral LVsegments (triangle).

$\square$ LGE $\square$ unenhanced myocardium $\square$ blood pool

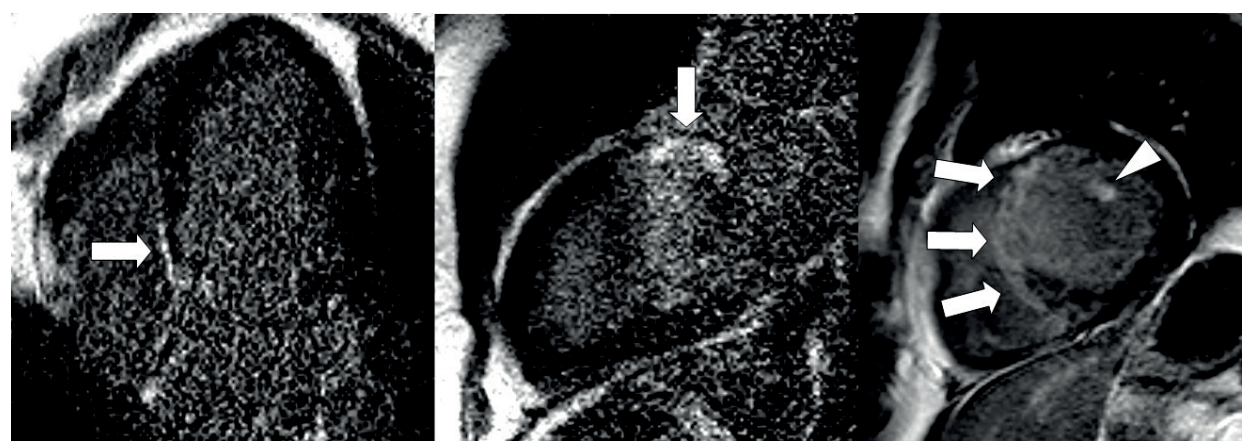

Figure 19. DECMR in a middle-aged female who presented with complete AVB and sVT demonstrates extensive transmural septal LGE (arrows) with papillary muscle involvement (triangle). 

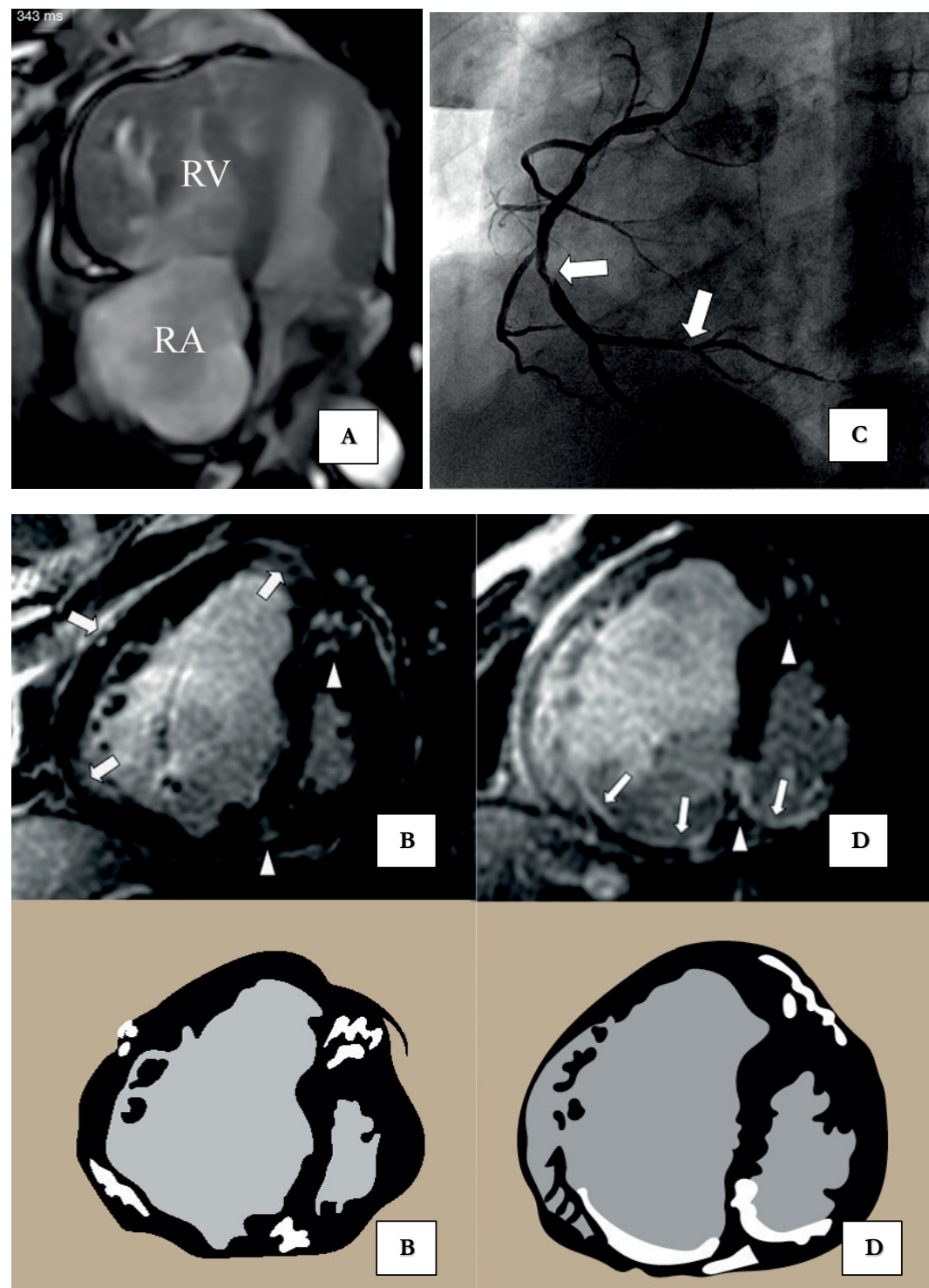

Figure 20. CMR study (Steady-State-Free Precession sequence, horizontal long axis view, end-diastolic frame), demonstrates dilation of the right ventricle (RV) and right atrium (RA), marked right ventricular hypertrophy, with displacement of the interventricular septum towards the left ventricle, both left ventricle and atrium are compressed (left-sided panel) (A) Contrast-enhanced CMR study (Inversion Recovery-Gradient Echo sequence, short axis view, end-diastolic frame) of the identical patient with pulmonary vascular sarcoidosis and resulting severe pulmonary arterial hypertension, demonstrates contrast-enhancement of the right ventricular hinge points (triangles) and free wall (arrows). (B) Two years later this patient presented with a subacute NSTEMI. The angiogram demonstrates stenosis with thrombus in segment two of the right coronary artery, and mid posterior descending artery. (arrows) (C) Follow-up contrast-enhanced CMR (Inversion-Recovery Gradient Echo sequence, short axis view, end-diastolic frame), demonstrates sub-endocardial enhancement in the distribution of the right coronary artery: the inferior right ventricular and left ventricular segments (arrows), different from the previously demonstrated ventricular insertion point enhancement (triangles). (right-sided panel) (D) 
A

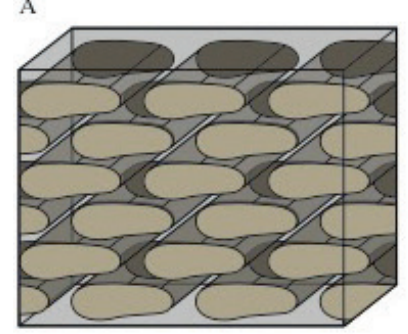

B
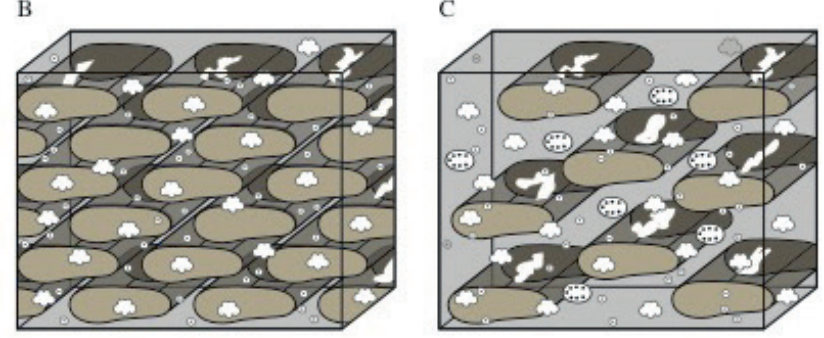

D

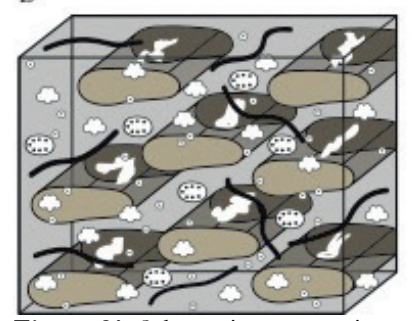

E

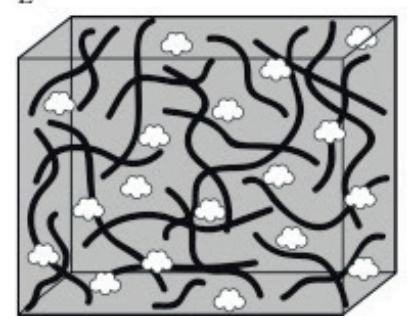

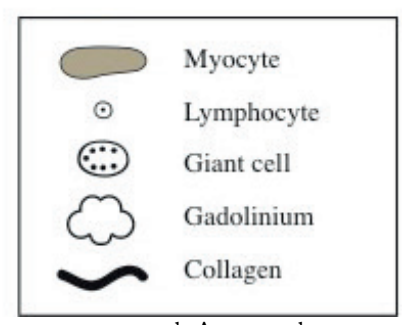

(

$\ldots$

Giant cell

Gadoliniun

Collagen

Figure 21. Schematic presentation of the LGE CMR principle - each box represents a voxel: A. normal myocardium before the administration of gadolinium, B. normal myocardium after the administration of gadolinium, distributed over the interstitial space, $\mathbf{C}$. interstitial expansion due to infiltration by inflammatory cells and edema, D. followed by deposition of collagen, E. replacement fibrosis representing focal scar. 


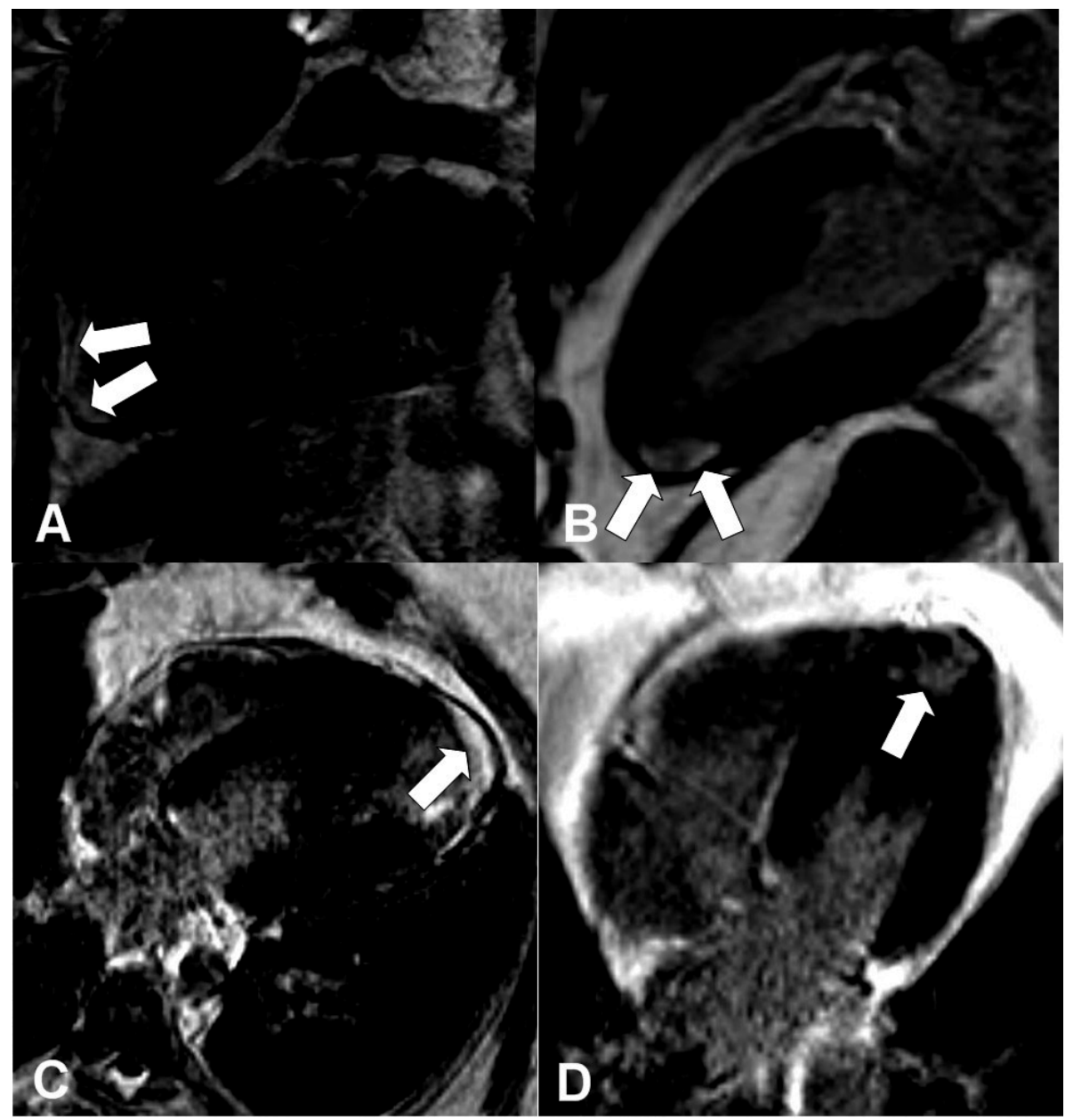

Figure 22. Delayed-enhanced three-dimensional breath-hold inversion-recovery gradient echo studies demonstrating confluent apical hyper-enhancement secondary to sarcoidosis (A: vertical long axis view, C: 4 chamber view) and apical patchy three-layer hyper-enhancement in a patient with coronary artery disease and myocardial infarction secondary to obstruction of the LAD (B: vertical long axis view, D: 4 chamber view). 


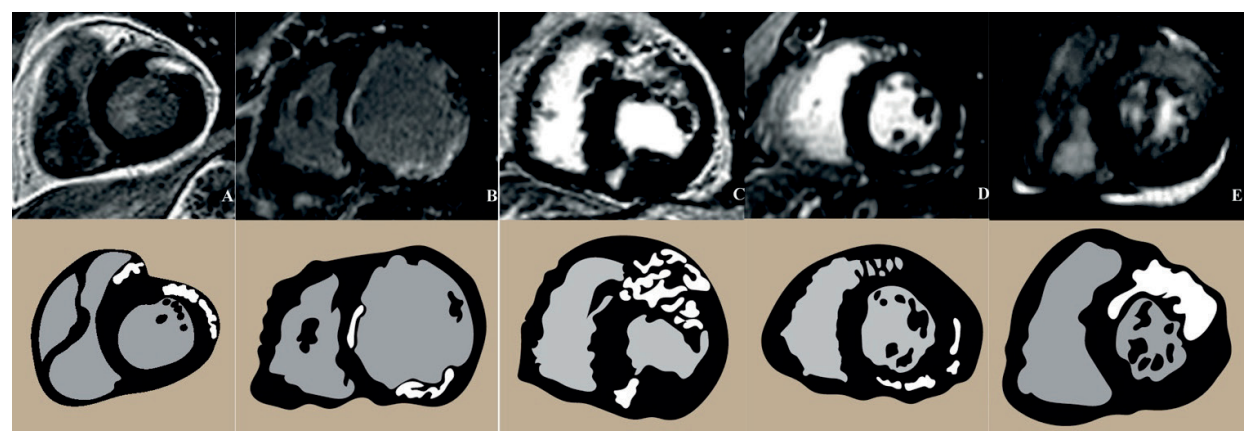

Figure 23. Gadolinium CMR (inversion-recovery gradient echo sequence, 10 minutes after the administration of $0.1 \mathrm{mmol} / \mathrm{kg}$ gadolinium-DTPA) may help differentiate between ischemic heart disease (A- previous lateral myocardial infarct, B - ischemic cardiomyopathy, subendocardial septal and posterolateral infarcts), hypertrophic cardiomopathy (C-scarring at the junctions of right and left ventricles), viral myocarditis (D- inferolateral epicardial LGE) and infiltrative cardiomyopathies (E-cardiac amyloidosis).

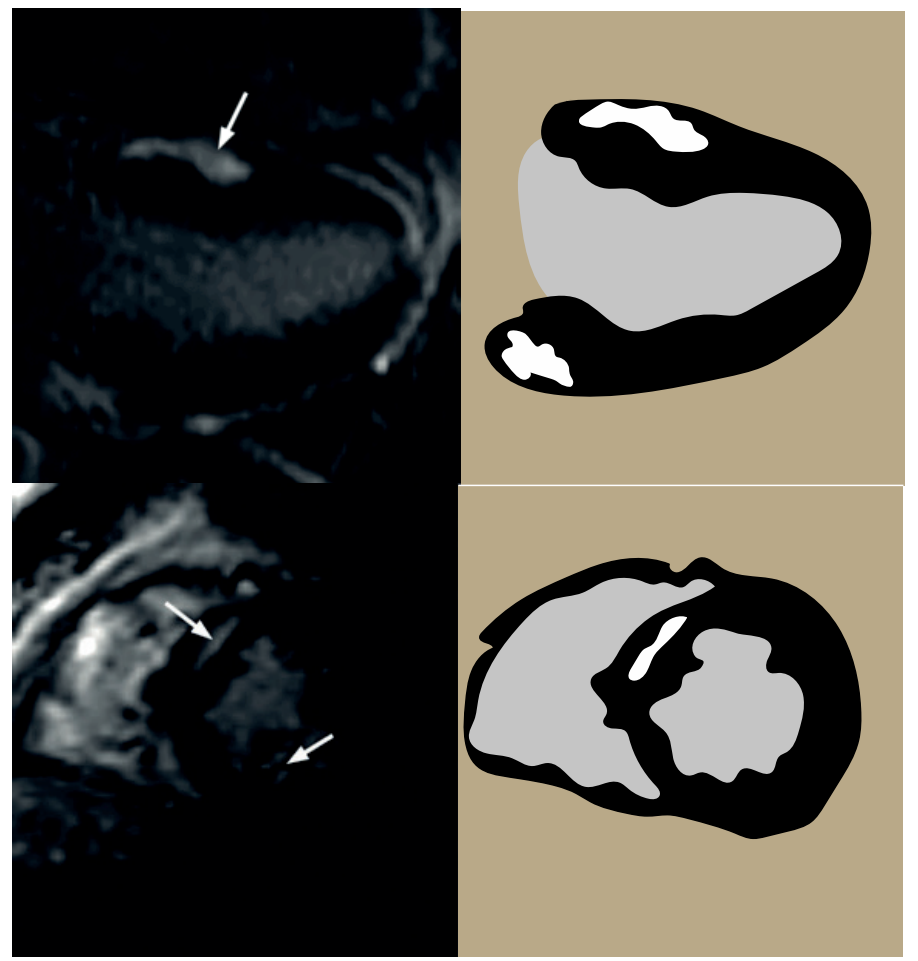

Figure 24. DECMR (IR-GRE, VLA and SA views) demonstrates antero-basal subepicardial, subepicardial inferior and mid-septal LGE in a middle-aged male who collapsed during a competitive field hockey game due to $\mathrm{VT} / \mathrm{VF}$.

$\square$ LGE $\square$ unenhanced myocardium $\square$ blood pool 


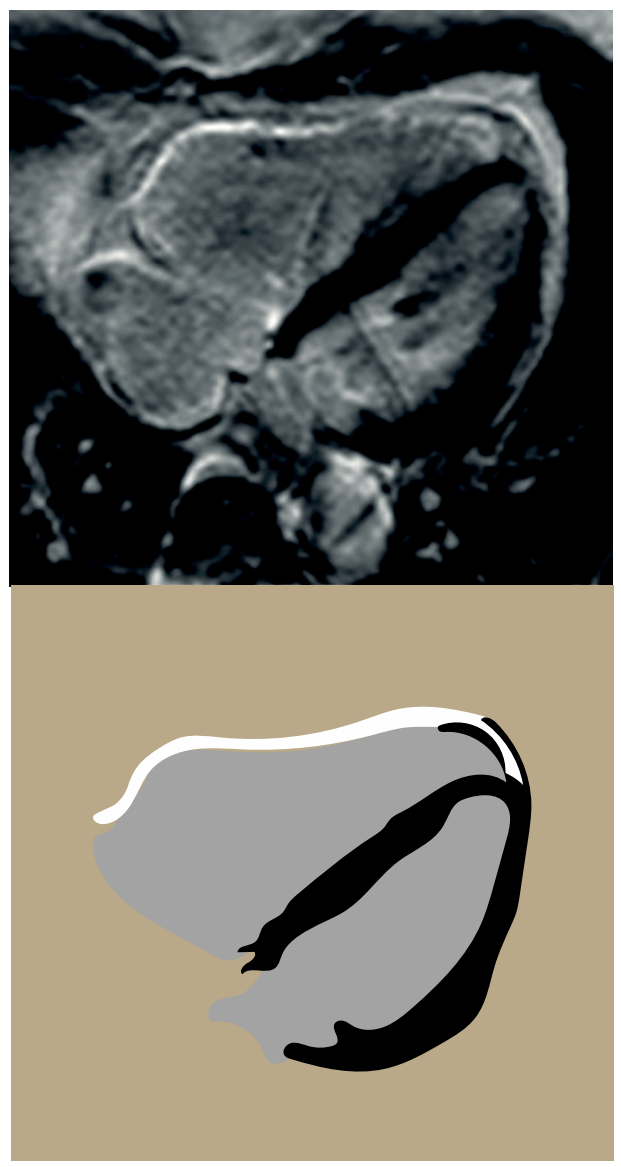

Figure 25. DECMR (IR-GRE, end-diastolic frame, HLA view) in a patient diagnosed with arrhythmogenic right ventricular cardiomyopathy, demonstrates dilation of the RV with LGE of the RV free wall. 


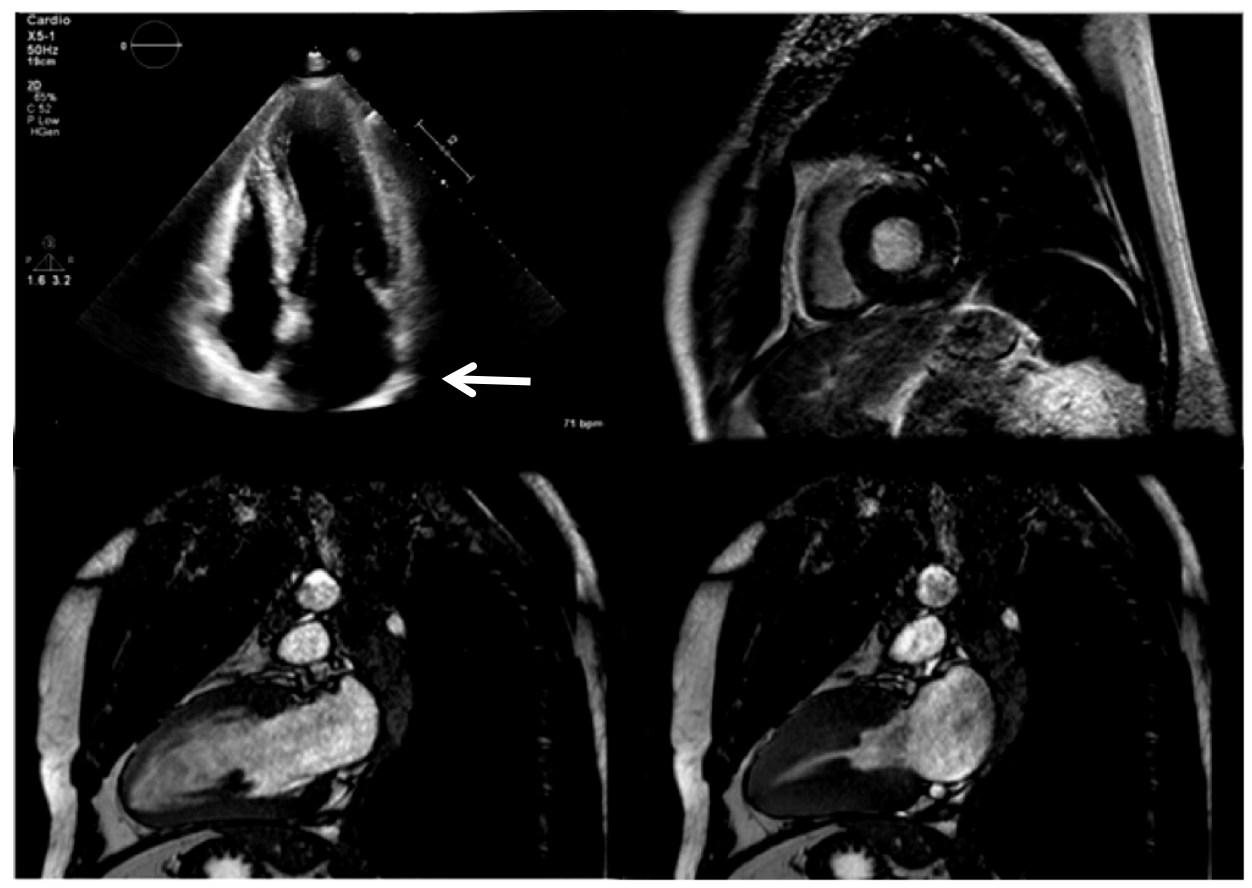

Figure 26. A 58-year-old male patient, previously diagnosed with pulmonary sarcoidosis, and asymmetric hypertrophy of the septum with systolic anterior motion (SAM), underwent a Morrow procedure for symptomatic LV outflow tract obstruction considered to be secondary to HCM. Histology of the resected basal septal tissue revealed non-caseating granulomas diagnostic of CS. (left-upper panel - 2 D TTE HLA demonstrating septal hypertrophy, right-upper panel -IRGRE patchy LGE in the interventricular septum and inferolateral LV segments, left and right lower panels - CMR SSFP, respectively diastolic and systolic VLA frames demonstrating severe hypertrophy of the basal interventricular : 'vtum ) (Matsumori A, Hara M, Nagai S, et al Hypertrophic cardiomyopathy as a manifestation of cardiac sarcoidc sis. Jap Circ J 2000;64(9):679-683. I kindly acknowledge the support of dr D van Kraaij who managed this patient and provided the images. 

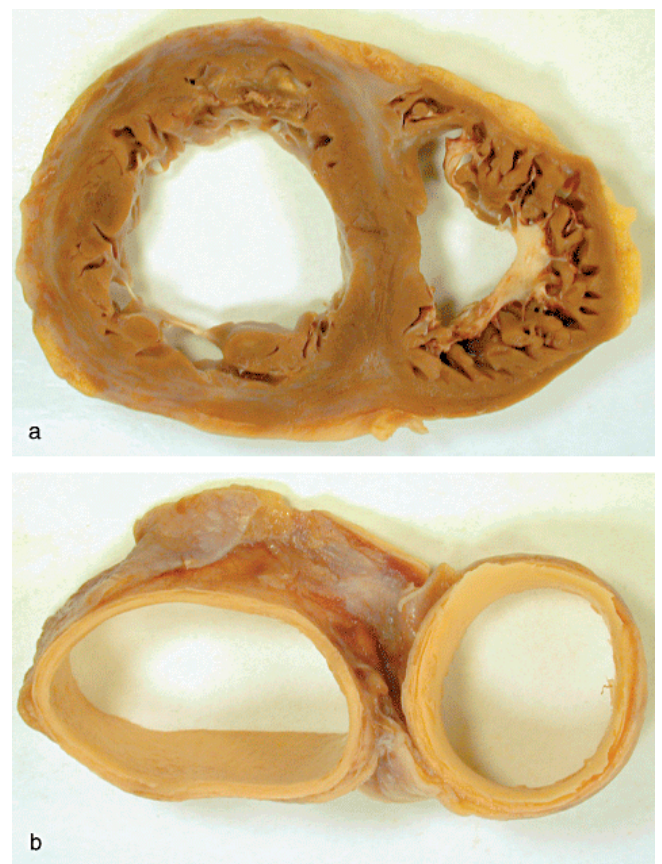

Figure 27. A patient with cardiac and pulmonary involvement by sarcoidosis. A The heart showed widespread fibrosis in association with granulomas (white patchy areas affecting the left and right ventricle (left side of the image) with right ventricular hypertrophy due to pulmonary arterial hypertension. B The pulmonary artery (left) shows a greater diameter than the aorta. Respirology 2010;16(1):69-77. Reproduced with permission by the publisher. 


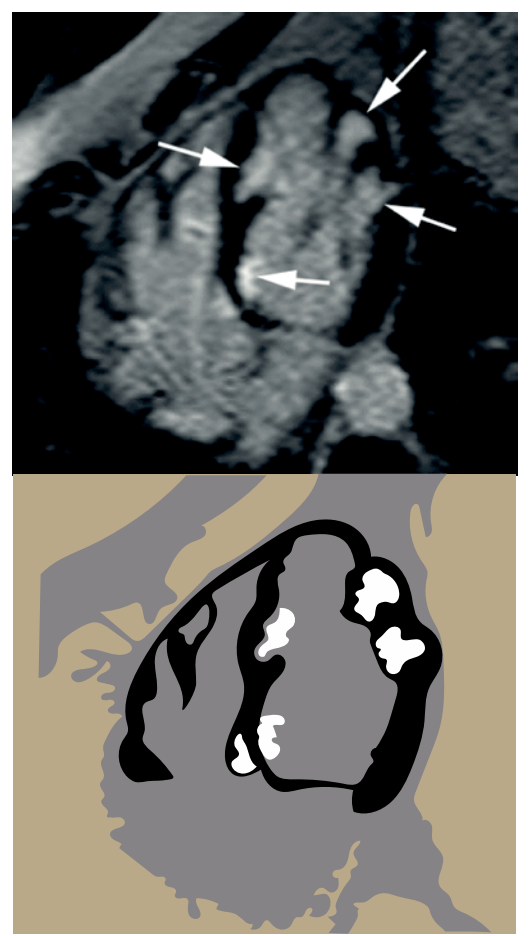

Figure 28. Delayed-enhanced CMR (horizontal long axis, IRGE) demonstrates multi-focal LGE in a nonischemic pattern, which has been associated with an increased risk for ventricular tachycardia.

(204.Crawford T, et al.Magnetic Resonance Imaging for identifying patients with cardiac sarcoidosis and preserved or mildly reduced left ventricular function at risk of ventricular arrhythmias. Circ Arrhythm Electrophys 2014;7(6):1109-1115. doi: 10.1161/CIRCEP.113.000156.) 


\section{All Cause Mortality}

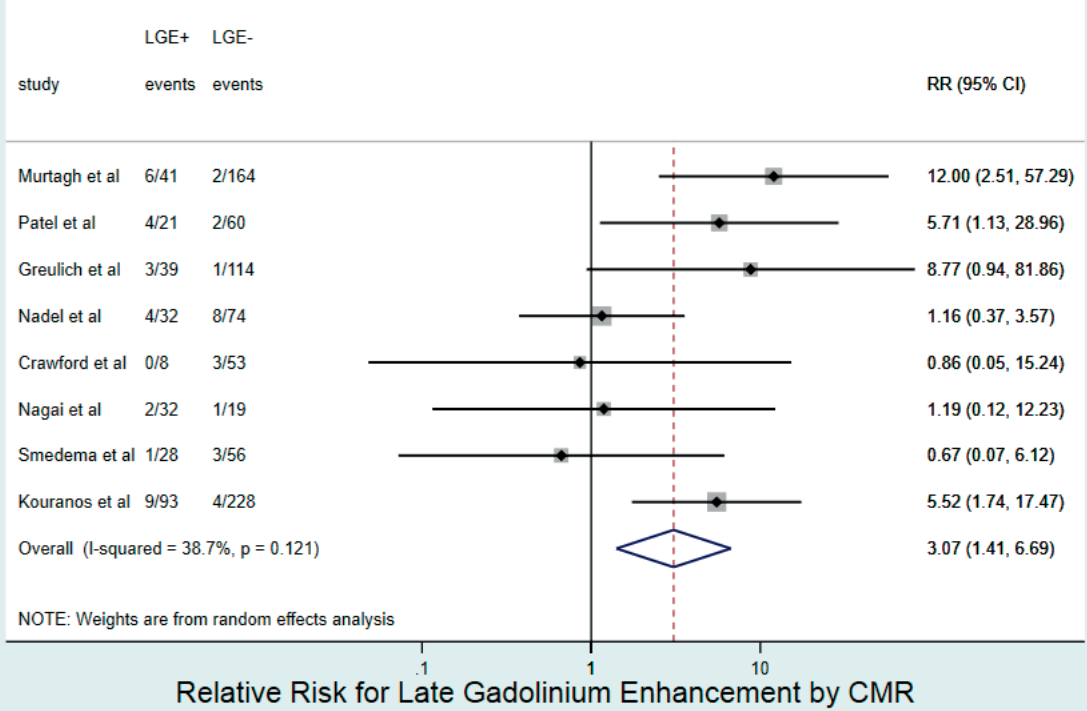

Figure 29. Pooled relative risk (RR) of any myocardial LGE for all cause mortality in sarcoidosis. $(\mathrm{p}=0.005)$

\section{Ventricular Arrythmia}

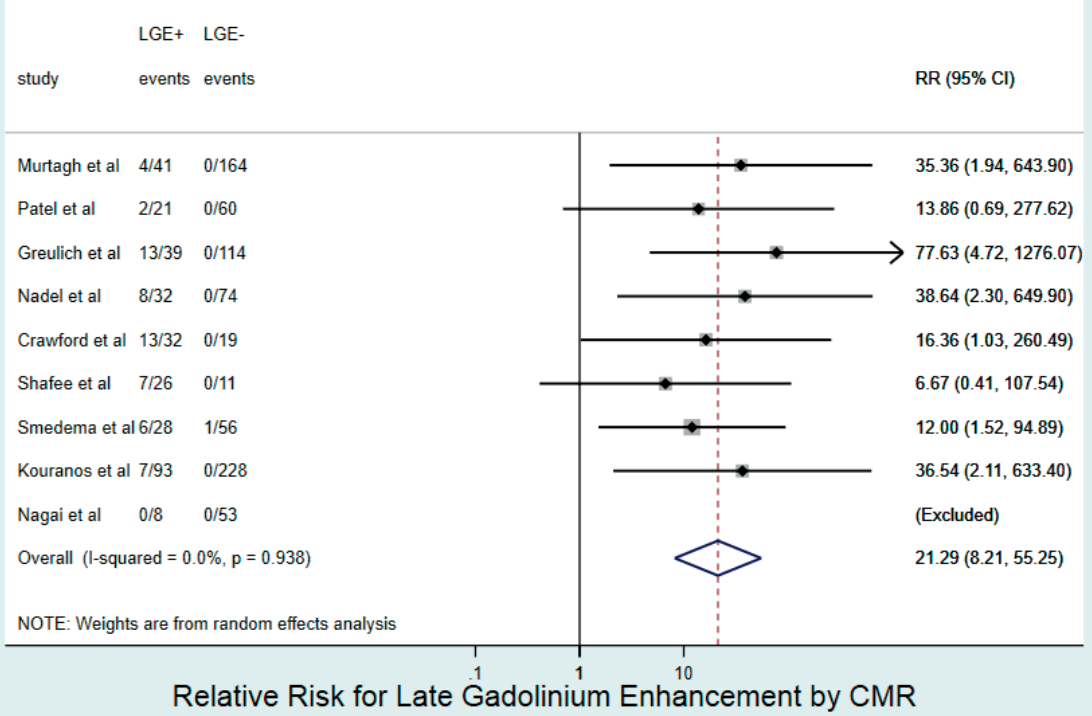

Figure 30. Pooled relative risk (RR) of any myocardial LGE for sustained ventricular tachycardia in sarcoidosis. $(\mathrm{p}<0.001)$ 


\section{Death or Ventricular Arrythmia}

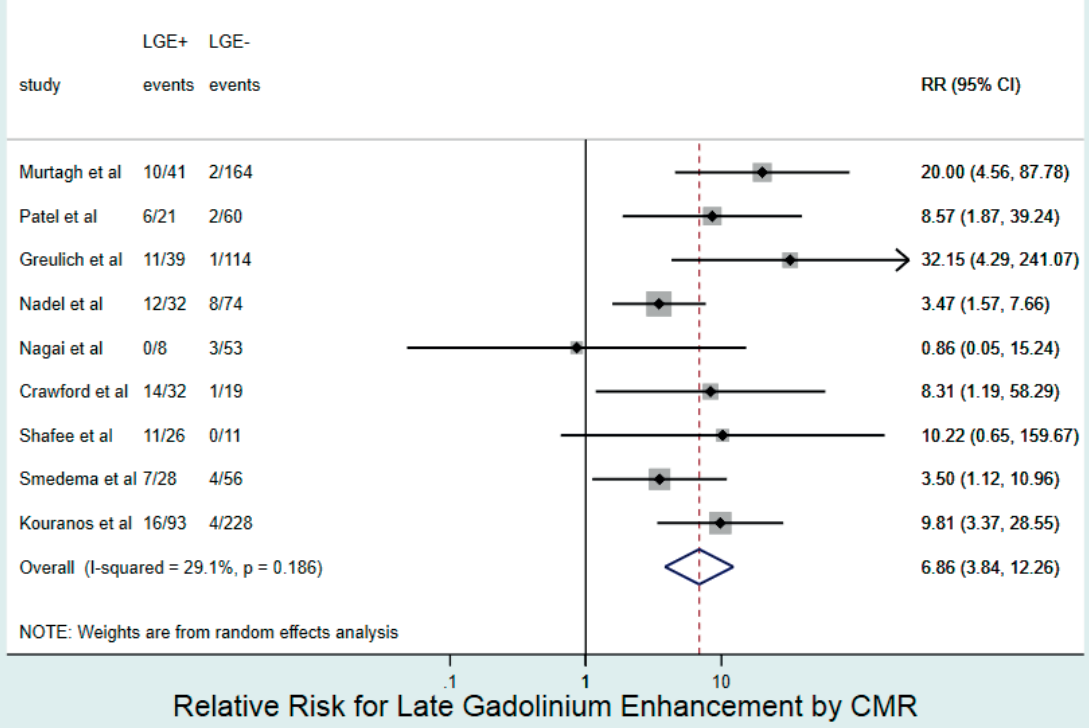

Figure 31. Pooled relative risk (RR) of any myocardial LGE for all-cause death or sustained ventricular tachycardia in sarcoidosis. $(\mathrm{p}<0.001)$

\section{Cardiovascular Mortality}

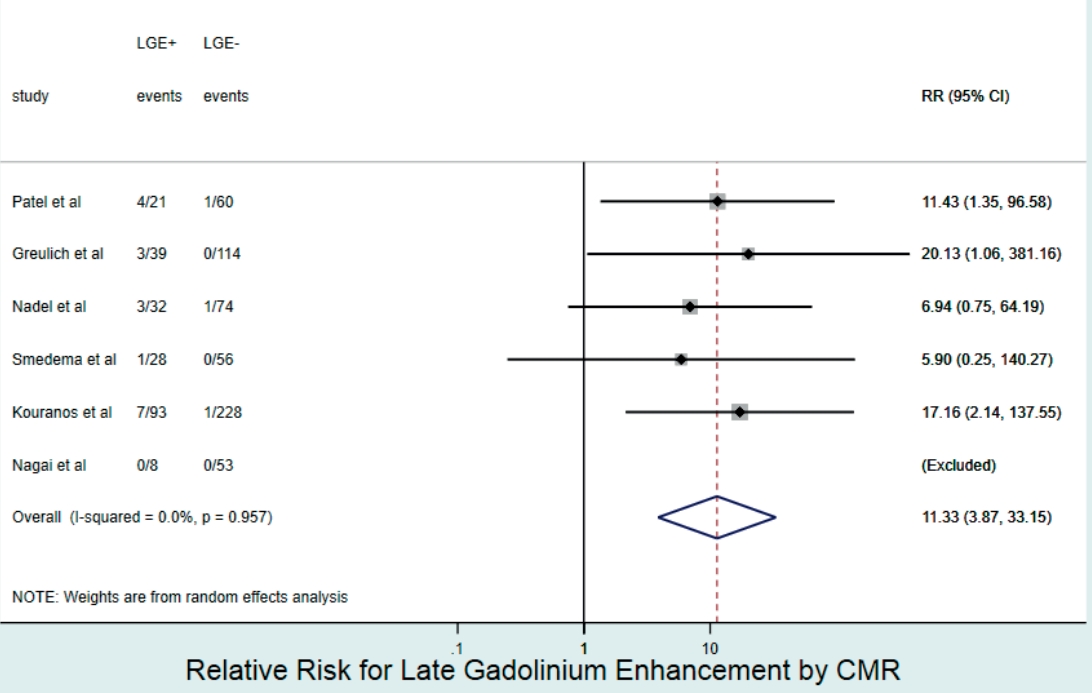

Figure 32. Pooled relative risk (RR) of any myocardial LGE for cardiovascular mortality in sarcoidosis. $(\mathrm{p}<$ $0.001)$ 


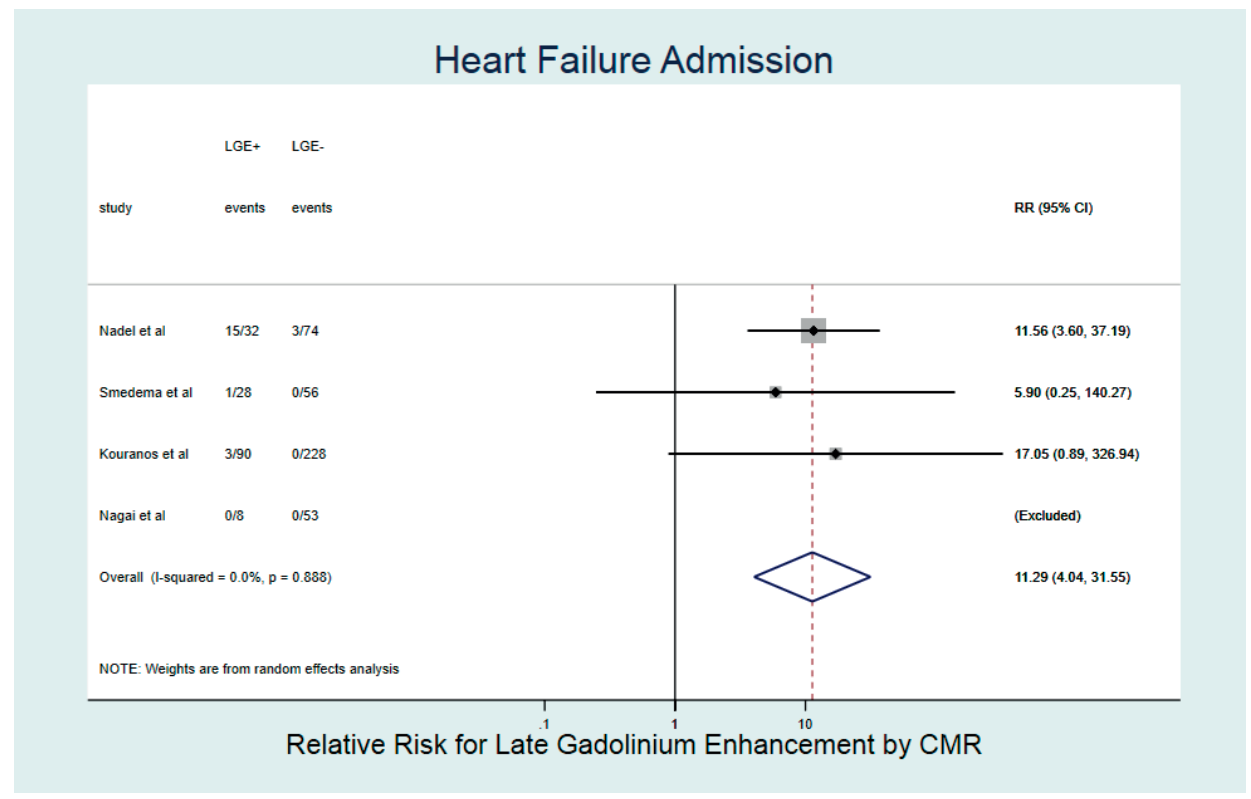

Figure 33. Pooled relative risk (RR) of any myocardial LGE for admission for congestive heart failure in sarcoidosis. $(\mathrm{p}<0.001)$

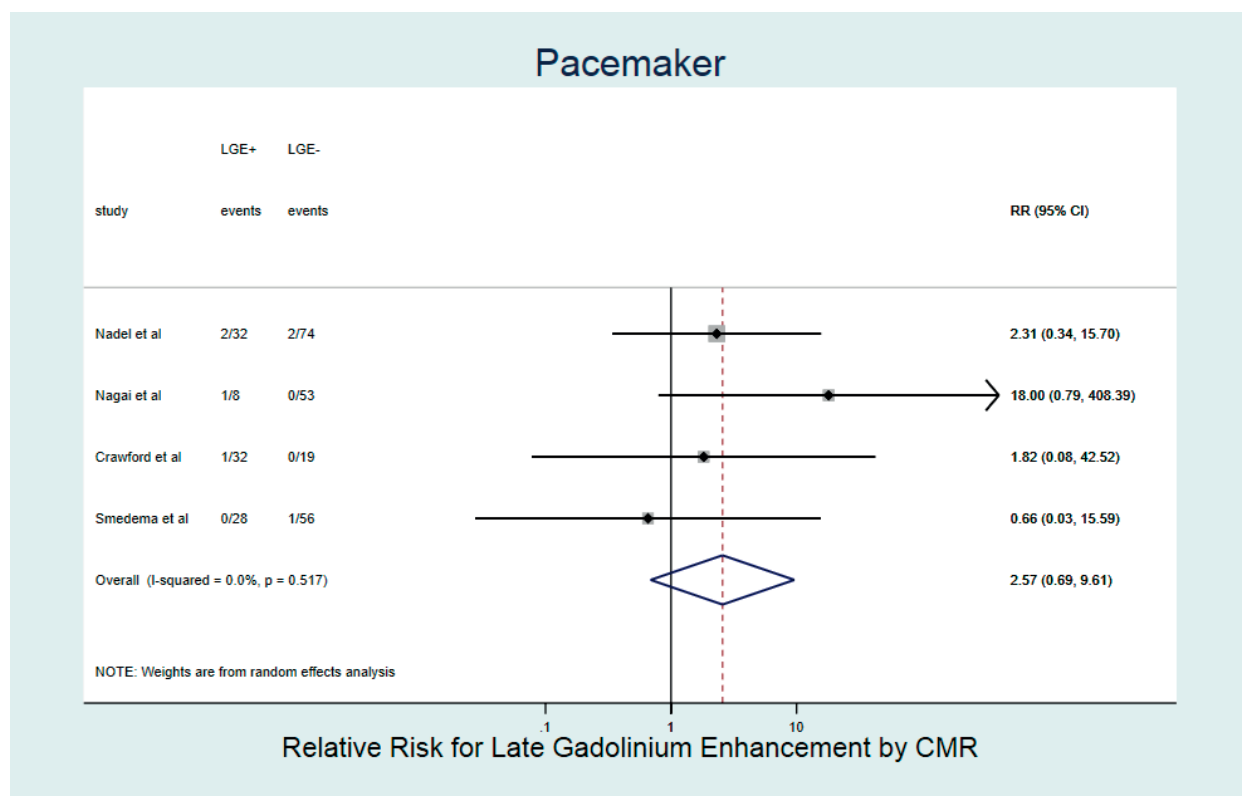

Figure 34. Pooled relative risk (RR) of any myocardial LGE for pacemaker implantation because of high degree atrio-ventricular block in sarcoidosis. $(\mathrm{p}=0.161)$ 


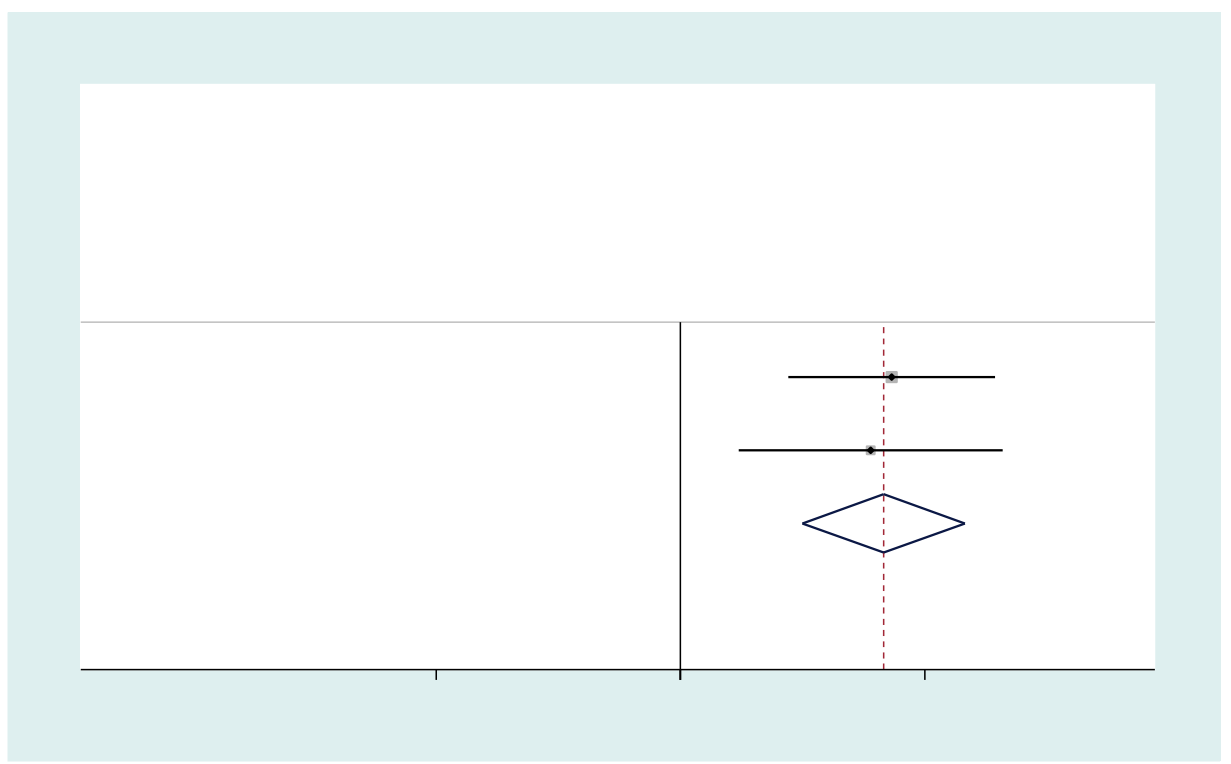

Figure 35. Pooled relative risk (RR) of RV LGE for sustained ventricular tachycardia in sarcoidosis. $(p<0.001)$

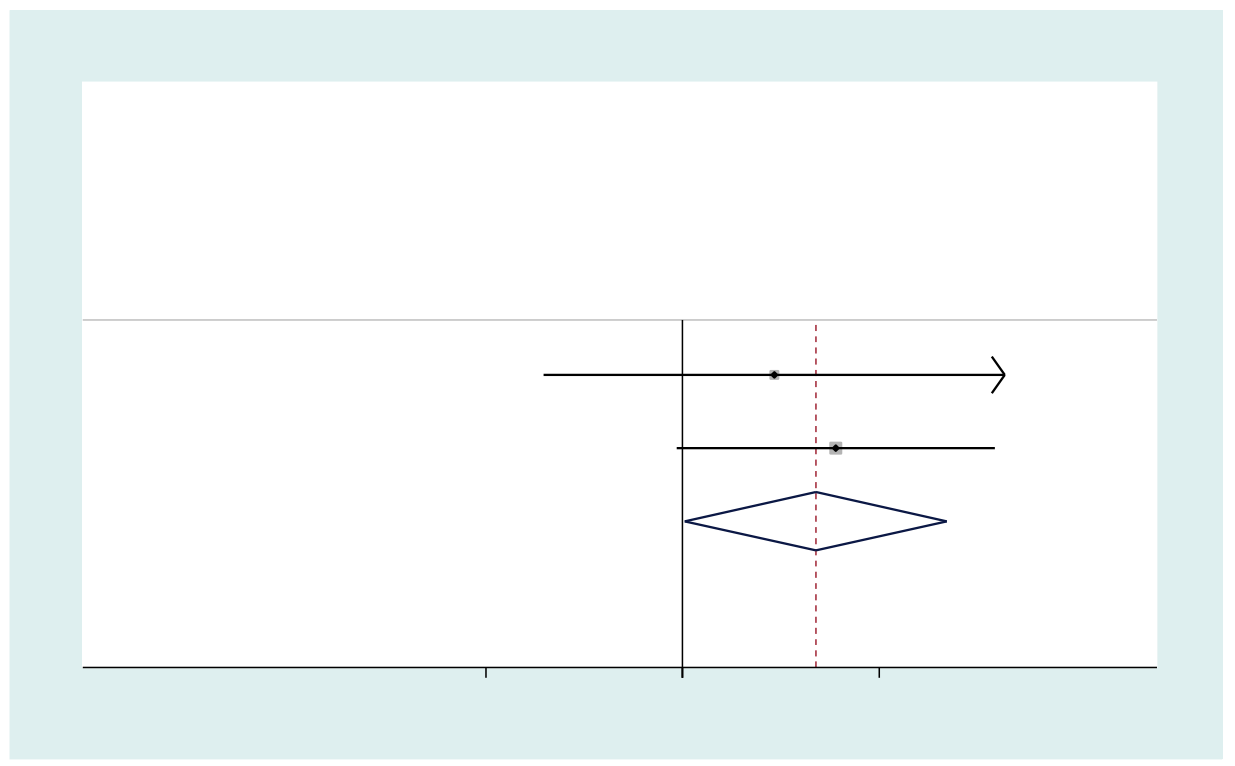

Figure 36. Pooled relative risk (RR) of any RV LGE for all cause mortality in sarcoidosis. $(\mathrm{p}=0.048)$ 


\section{Chapter 3 \\ Cardiac involvement in patients with pulmonary sarcoidosis assessed at two University Medical Centres in The Netherlands}

Chest 2005;128:30-35.

J.P.Smedema MD, MMed(Int), G.Snoep MD, M.P.G. van Kroonenburgh MD, PhD, R.J. van Geuns, MD, PhD, W.R.M. Dassen, PhD, A.P. Gorgels, MD, PhD, H.J.G.M. Crijns, $\mathrm{MD}, \mathrm{PhD}$.

Departments of Cardiology (JPS, HJGMC, APG), Radiology (GS), Nuclear Medicine (MvK), University Hospital Maastricht, Dr Debyelaan 25, 6202 AZ, Maastricht, The Netherlands. Department of Cardiology and Radiology (RJvG), Erasmus Medical Centre, Rotterdam. 


\section{Structured abstract}

Study objectives: We aimed to determine cardiac involvement in patients with pulmonary sarcoidosis (PS) followed up at two University Medical Centres in The Netherlands.

Design: We reviewed the findings in consecutive patients who had been assessed by our departments during 1998 - 2004, and divided them in patients who had presented with symptoms of cardiac sarcoidosis (CS) (group A), and those who had been screened for this condition (group B).

Setting: Two University Medical Centers in the Netherlands.

Patients: 101 patients (69 males, mean age 47.6 years, 32 females, mean age 47.3 years) with biopsy proven pulmonary sarcoidosis.

Interventions: 12-lead ECG (101), ambulatory ECG (74), echocardiography (80), ${ }^{201}$ thallium SPECT (61), cardiac magnetic resonance imaging (87), coronary angiography to exclude coronary artery disease (17), and endo-myocardial biopsy (9).

Measurements: Electrocardiographic, structural and functional cardiac abnormalities according to the modified guidelines of the Japanese Ministry of Health and Welfare (1993).

Results: Sixteen of the 19 patients of group A and 3 of the 82 of group B were diagnosed with CS. During a mean follow up of 1.7 years ( 3 months - 4 years) 4 patients of group A died (20\%), and 9 had a pacemaker and/or ICD implanted (47\%), while the patients of group B had an uncomplicated course.

Conclusions: Once PS patients have developed symptomatic CS, their prognosis becomes very grim. In contrast, the prognosis in asymptomatic cardiac involvement in PS patients is good. Considering the poor prognosis of symptomatic CS, pulmonologists should consider regular screening of their PS patients for cardiac involvement with straightforward detection methods.

\section{Contents}

Structured abstract $\quad 98$

3.1 Introduction 99

3.2 Patients and methods 99

3.3 Results 101

3.4 Discussion 102

$\begin{array}{lll}3.5 & \text { Study limitations } & 103\end{array}$

3.6 Conclusions 103

3.7 Acknowledgements 104

References 105 


\subsection{Introduction}

Sarcoidosis is a multi-system granulomatous disorder of unknown etiology with symptomatic cardiac involvement in approximately five percent of patients. $(1,2)$

The clinical features of cardiac sarcoidosis (CS) include congestive heart failure, cor pulmonale, supraventricular- and ventricular arrhythmias, conduction disturbances, ventricular aneurysms, pericardial effusion and sudden death. (3) The diagnosis of CS is made in the co-existence of non-caseating granulomas on myocardial biopsy or biopsies of any extra-cardiac tissue (with the exclusion of other causes for granulomatous inflammation such as mycobacterial or fungal infection) and cardio-vascular abnormali-ties for which other possible causes have been excluded. The guideline from the Japanese Ministry of Health and Welfare provides an excellent diagnostic frame work. (4) (Table 1) The prevalence of sarcoidosis in The Netherlands is estimated to be 20-30 per 100 000, whilethe prevalence of cardiac involvement is unknown. Postmortem studies revealed cardiac involvement in twenty to thirty percent of patients with sarcoidosis in the United States. $(5,6)$ In Japan cardiac involvement is reported to be present in as many as $58 \%$ of patients, and is responsible for as many as $85 \%$ of deaths from sarcoidosis. $(7,8)$ We aimed to determine cardiac involvement in sarcoidosis patients followed up at two University Medical Centres in The Netherlands, and reviewed the findings in consecutive patients who had presented with symptoms of cardiac sarcoidosis, and those who had been screened for this condition.

\subsection{Patients and methods}

\section{Study population}

Between July 2000 and May 2004, 101 patients (69 males (33-71, mean 47.6 years), 32 females (29-72, 47.3 years), Caucasian: 87 (85\%), African: 4, Asian: 9, Middle-Eastern: 1) with histologically proven pulmonary sarcoidosis underwent cardiac assessment in the cardiology departments of , the Erasmus Medical Centre (16) and University Hospital Maastricht (85). The diagnosis of sarcoidosis was confirmed if the clinical presentation and chest radiogragraphic finding were supported by histological evidence of non-caseating granulomas by transbronchial biopsy, and the possibility of infection, environmental factors or hypersensitivity reaction to medication causing granulomatous inflammation, had been eliminated. Patients had either presented with symptoms suggestive of cardiac sarcoidosis $(n=19)$, or had been screened for cardiac involvement $(n=82)$. Patients underwent clinical assessment including determination of highest serum levels of angiotensin converting enzyme (SACE) ( $\mathrm{n}=64)$, 12-lead electrocardiograms $(\mathrm{n}=101)$, ambulatory electrocardiographic monitoring ( $\mathrm{n}=74)$, radiological chest stage by X-ray and/or High Resolution CT ( $\mathrm{n}=101)$, transthoracic echocardiograms $(\mathrm{n}=80),{ }^{201}$ thallium

single-photon- emission-computed tomography (SPECT) ( $\mathrm{n}=61)$, and gadolinium-DTPA cardiac magnetic resonance imaging (CMR) (87). Of the $88 \mathrm{CMR}$ studies 5 were discarded because of insufficient image quality. Seventeen patients underwent diagnostic coronary angiography to exclude coronary artery disease, in six patients endo-myocardial biopsies 
were taken, and three patients had a post-mortem examination. Because of the limited diagnostic yield of endomyocardial biopsies, and the invasive nature with an associated risk of morbidity, we felt not justified to systematically subject patients who were screened for CS to this procedure. For the purpose of the present study the Japanese guideline was modified by excluding endomyocardial biopsy as a diagnostic parameter. Since the guidelines were compiled before CMR had become integral part of cardiac assessment, we further modified our diagnostic scheme by incorporating CMR assessment of wall thickness and motion. The diagnosis of CS, according to the modified guideline (Table 1) was made in 19 of 101 patients included in the study.

\section{Electrocardiography and ambulatory electrocardiography}

A 12-lead surface electrocardiogram (ECG) was made (MAC, Marquette, Milwaukee, Wisconsin; paper speed $25 \mathrm{~mm} / \mathrm{sec}$ ) and the findings were classified as abnormal i.e. in keeping with the ECG criteria of the guidelines (Table1). Ambulatory ECGs (74) were performed for $24-72$ hours, and were considered abnormal when evidence of intermittent atrio-ventricular conduction delay or block, intermittent bundle branch blocks or ventricular arrhythmias such as frequent monomorphic and/or polymorphic PVCs $>100 / 24$ hours, non-sustained VTs, and/or sustained VTs were found.

\section{Echocardiography}

Studies were performed with a Sonos 5500 phased array imaging system (Hewlett Packard) and considered abnormal when regional or global systolic dysfunction, wall thickening or thinning was found.

\section{Thallium myocardial scintigraphy}

After treadmill peak exercise, or during intravenous infusion of dipyridamol, ${ }^{201}$ thallium was administered and SPECT performed on a Siemens triple-detector gamma camera (MultiSPECT-3), equipped with low-energy, high resolution collimators. The images were made in a $64 \times 64$ matrix ( 60 frames $/ 45$ seconds). The thallium scan was considered suggestive of CS when areas with reversed uptake and/or irreversible perfusion defects were present, and/or reversible perfusion defects were found in patients with normal coronary arteries at angiography. Regional defects were localized according to the 17 segment 1 model (9)

\section{Cardiac Magnetic Resonance}

Studies were performed using a 1.5 Tesla MRI scanner (Philips, Best, The Netherlands and General Electric, Milwaukee, Wisconsin, USA) with a cardiac-dedicated phased-array coil. The CMR studies were ECG triggered by standard software, and obtained in diastole to minimize artefact due to cardiac motion. Studies consisted of multislice-multiphase steady- 
state-free precession (SSFP), spin echo (SE) and fat saturated T2 weighted breath hold sequences of the short axis (SA), vertical long axis (VLA) and four chamber (4CH) views. SSFP sequences were performed to assess regional wall motion abnormalities. T2 weighted studies were performed to assess the presence of myocardial inflammation. Ten minutes after the additional administration of $0.1-0.2 \mathrm{mmol} / \mathrm{kg}$ gadolinium-DTPA (Schering, Berlin), a SE (slice thickness $8 \mathrm{~mm}$, gap $.8 \mathrm{~mm}$, matrix $512 \times 512$, FOV $360 \mathrm{~mm}$ ) and/or 3-D inversion recovery-gradient echo (IR-GRE) breath hold sequence (SA, VLA, $4 \mathrm{CH}$ ) (slice thickness $10 \mathrm{~mm}$, no gap, matrix $256 \times 256$, FOV $400 \mathrm{~mm}$ ) was used to assess for the presence of contrast enhancing lesions. The inversion time $(250-400 \mathrm{msec})$ was determined on an individual base to obtain optimal nulling of the un-enhanced myocardial signal. Regional differences in left ventricular wall enhancement were measured and localized according to the 17 segment model (9) (MASS suite post processing software, MEDIS, Leiden). The total time required for the investigation was 30-45 minutes.

The studies were independently evaluated by 4 blinded observers, 3 cardiologists and 1 radiologist, with experience in CMR. The studies were considered to be abnormal when at least 2 observers described identical abnormalities.

\section{Coronary angiography}

Diagnostic coronary angiography was performed when considered indicated by the managing physician, and was generally done in patients with symptoms and findings suggestive of significant coronary artery disease. One patient underwent diagnostic coronary angiography as part of the diagnostic work up before cardiac transplantation.

\section{Statistical analysis}

All statistical analyses were performed using the statistical software package SSPS version 11.5. Group data are expressed as mean \pm SD. Continuous variables were assessed using the parametric $t$ test for independent samples or Mann Whitney test were appropriate, and all categorical variables were assessed using the chi-square test. Statistical significance was defined as a $\mathrm{p}$ value less than 0.05 .

\subsection{Results}

\section{Patient characteristics}

The demographic and clinical characteristics of group A and group B are presented in Table 2. Significantly more patients in group A were diagnosed with CS compared to group B. Pulmonary involvement based on radiological chest stage was significantly more extensive in group B, while the degree of functional impairment was significantly higher in group A. 


\section{Findings at cardiac assessment}

The findings at cardiac assessment are presented in Table 2, with Tables 3 and 4 presenting more detailed information on the findings in all patients diagnosed with CS. The ECGs, ambulatory ECGs, echocardiograms and CMR studies revealed significantly more conduction abnormalities, ventricular arrhythmias and regional loss of left ventricular wall thickness and function in group A. Of the diagnostic techniques, the ECG and CMR showed most abnormalities. The ECG demonstrated conduction abnormalities in 10 of the 16 patients $(63 \%)$ diagnosed with CS. In 4 patients of group B electrocardiographic signs of right ventricular hypertrophy were present.CMR and/or echocardiography diagnosed decreased systolic left ventricular function (mean 35\%, range 20-56\%) with global or regional wall motion abnormalities in $12(75 \%)$ of the CS patients. In 10 of the 16 CS patients CMR and SPECT were available. In 4 patients CMR and SPECT diagnosed defects, while CMR revealed small defects in 4 patients with normal SPECT studies. The findings with SPECT were not significantly different between group A and B. In 8 patients of group B small (ir)reversible perfusion defects were detected, which were mainly located in the inferior left ventricular (LV) wall. SPECT demonstrated reversible perfusion defects in the anterior wall in 2 patients with coronary artery disease (CAD) of group $\mathrm{B}$ and an irreversible perfusion defect in this region in 1 patient with $\mathrm{CAD}$ of group A.Coronary angiography was performed in 17 patients, and revealed significant coronary artery disease in 2 patients of group A. Two patients were diagnosed with hypertrophic cardiomyopathy, one of who was also diagnosed with CS.

\section{Clinical data at follow up}

The management, duration of follow up and outcome during follow up are presented in Tables 3 and 4. Of the 16 patients diagnosed with CS in group A, 9 received a pacemaker and/or implanttable cardioverter/defibrillator (ICD). Three patients died of ventricular arrhythmias, one of treatment resistant VTs after implantation of an ICD, and one patient died of complications after cardiac transplantation. Nine patients were treated with immune-suppressive drugs, corticosteroids with methotrexate or azathioprine. None of the patients of group B had significant cardiovascular complications or died during follow up.

\subsection{Discussion}

Our study is the first to systematically evaluate cardiac involvement in patients with sarcoidosis in The Netherlands, and the largest to employ CMR. Earlier studies in the Sweden (86 patients), Ireland (50 patients), Israel (42 patients), the USA (88 patients), Japan (41 patients) and France (50 patients) used ECG, echocardiography, ${ }^{201}$ thallium SPECT and CMR in prospective studies evaluating the prevalence of cardiac involvement in referred patients with sarcoidosis (10-18) Abnormalities were found in up to $23 \%$ of European and $63.4 \%$ of Japanese patients. In our study, depending on the technique used, abnormalities were found in 10 to $26 \%$ of patients without cardiac symptoms. SPECT detected small 
irreversible perfusion defects in 13 patients, while gadolinium (Gd)-enhancing lesions were present in 12 patients. Both SPECT and CMR demonstrated lesions in 4 patients. Wagner et al. have previously demonstrated the ability of Gd-enhanced CMR to diagnose even small amounts of myocardial scar tissue not detected by SPECT in patients with coronary artery disease. (19) Histological assessment of gadolinium-DTPA (Gd) enhanced myocardium has been correlated with fibrosis and active myocarditis. $(\mathbf{2 0 , 2 1 , 2 2 )}$ Since T2 weighted studies revealed increased signal, signifying inflammation, in only 1 patient, delayed enhancement in our patients suggest the presence of myocardial scar tissue. The favourable prognosis of abnormal SPECT studies in asymptomatic sarcoidosis patients was demonstrated by Kinney et al. in 52 similar patients in whom the presence of of perfusion defects diagnosed with SPECT did not predict survival during a mean follow up of 89 months. (23) Since CMR is a relatively new diagnostic technique, and experience in evaluating patients with sarcoidosis limited, long-term follow up will have to determine the significance of small gadolinium-enhancing lesions in asymptomatic patients. The significantly poorer functional NYHA class in patients of group A, despite less radiological pulmonary involvement, is explained by more extensive LV involvement, resulting in a lower ejection fraction, heart failure and ventricular arrhythmias. When considering the study of Yazaki et al., who determined with multivariate analysis that the presence of higher NYHA class, increased left ventricular end-diastolic diameter and sustained VTs were independent predictors of death, we anticipated poorer outcome of group A. (24) Although the introduction of the ICD is expected to improve outcome in patients with CS, during a mean follow up of 15 months ( 3 to 54 months) in our population it delivered therapy in only 1 of 6 patients that had received the device. (25)

\subsection{Study limitations}

Theoretically bias was introduced by evaluating a pre-selected patient population that had been referred to tertiary centres. This however seems unlikely when considering the fact that only few of the patients who were screened for CS were actually diagnosed with this condition. It seems more likely that cardiac involvement may have been underestimated, since the diagnosis was based on the modified guideline of the Japanese Ministry of Health and Welfare. In the absence of diagnostic cardiac histology, CS could only be diagnosed in the presence of ECG abnormalities. As previously published by Silverman et al, and demonstrated by patients 1 and 3 (Table 3), cardiac involvement may well be present in the absence of diagnostic ECG abnormalities.

\subsection{Conclusions}

In our cohort of 101 pulmonary sarcoidosis patients, the rate of cardiac involvement in those screened for this condition was low (4\%) and the prognosis was good. The CS patients who presented with cardiac failure or ventricular arrhythmias had significant morbidity and a mortality rate of $25 \%$ during a mean follow up of 15 months. Based on the 


\section{Chapter 3}

findings of our current study, we recommend regular electrocardiographic evaluation in patients with PS and early referral for additional cardiac assessment with echocardiography or CMR in patients with unexplained fatique, dyspnoea, or palpitations.

\subsection{Acknowledgements}

The authors gratefully acknowledge Dr H.Kuehl, MD, PhD, Aachen University Medical Centre, and Dr A.M.Beek, MD, Free University Medical Centre, Amsterdam, for assessing the CMR studies. 


\section{References}

1. Hagemann GJ, Wurm K. The clinical, electrocardiographic and pathological features of cardiac sarcoid. In: Jones Williams W, Davies BH, eds. Sarcoidosis and other granulomatous disorders. Proceedings of the 8th International Conference. Cardiff: Alfa Omega Publishing, 1980:601-606.

2. Johns CJ, Michele TM. The clinical management of sarcoidosis: a 50-year experience at the Johns Hopkins Hospital. Medicine 1999;78:65-111.

3. Deng JC, Baughman RP, Lynch JP. Cardiac involvement in sarcoidosis. Sem Resp Crit Care Med 2002;23:513-526.

4. Hiraga H, Yuwai K, Hiroe M, et al. Guideline for the diagnosis of cardiac sarcoidosis: study report on diffuse pulmonary diseases [in Japanese]. Tokyo, Japan, The Japanse Ministry of Health and Welfare. 1993;23-24.

5. Silverman KJ, Hutchins GM, Bulkley BH. Cardiac sarcoid: a clinico-pathologic study of 84 unselected patients with systemic sarcoidosis. Circulation 1979;58:1204-1211.

6. Longscope WT, Freiman DG. A study of sarcoidosis: based on a combined investigation of 160 cases, includng 30 autopsies from Johns Hopkins Hospital and Massachusetts General Hospital. Medicine 1952;31:1-132.

7. Matsui Y, Iwai K, Tachibana T, et al. Clinicopathological study on fatal myocardial sarcoidosis. Ann NY Acad Sci 1976;278:455-469.

8. Tachibana T, Iwai K, Takemura T. Study on the cause of death in patients with sarcoidosis in Japan [abstract]. XII World Congress on Sarcoidosis, Kyoto, Japan, September 8-13,1991.

9. Cerqueira MD, Weissman NJ, Dilsizian V, et al. AHA writing group on myocardial segmentation and registration for cardiac imaging. Standardized myocardial segmentation and nomenclature for tomographic imaging of the heart. Circulation 2002;105:539-542.

10. Thunell M, Bjerle P, Sterjnberg N. ECG abnormalities in patients with sarcoidosis. Acta Med Scand 1983;213:115-118.

11. Suzuki T, Kanda T, Kubota S, et al. Holter monitoring as non-invasive indicator of cardiac involvement in sarcoidosis. Chest 1994;106:1021-1024.

12. Fahy GJ, Marwick T, McGreery CJ, et al. Doppler echocardiographic detection of left ventricular diastolic dysfunction in patients with pulmonary sarcoidosis. Chest 1996;109: 62-66.

13. Lewin RF, Mor R, Spitzer S, et al. Echocardiographic evaluation of patients with systemic sarcoidosis. Am Heart J 1985;110:116-122.

14. Burstow DJ, Tajik J, Baily KR, et al. Two-dimensional echocardiographic findings in systemic sarcoidosis. Am J Cardiol 1989;63:478-482.

15. Yamamoto N, Gotoh K, Yagi Y, et al. Thallium-201 myocardial SPECT findings at rest in sarcoidosis. Ann Nucl Med 1993;7:97-103.

16. Vignaux O, Dhôte R, Duboc D, et al. Detection of myocardial involvement in patients with sarcoidosis applying T2-weighted, contrast-enhanced and cine magnetic resonance imaging: initial results of a prospective study. J Comput Assist Tomograph 2002;26:762-767.

17. Dhôte R, Vignaux $\mathrm{O}$, Blanche $\mathrm{P}$, et al. Value of MRI for the diagnosis of cardiac involvement in sarcoidosis. Revue Med Int 2003;24:151-157.

18. Sköld CM, Larsen FF, Rasmussen E, et al. Determination of cardiac involvement in sarcoidosis by MRI and Doppler echocardiography. J Int Med 2002;252:465-471.

19. Wagner A, Mahrholdt H, Holly TA, et al. Contrast-enhanced MRI and single photon emission computed tomography (SPECT) perfusion imaging for the detection of subendocardial myocardial infarcts: an imaging study. Lancet 2003;361:374-379.

20. Mahrholdt H, Goedeke C, Wagner A, et al. CMR in human myocarditis: a comparison to histology and molecular pathology. Circulation 2004;16:1250-1258.

21. Aso $\mathrm{H}$, Takeda $\mathrm{K}$, Ito $\mathrm{T}$, et al. Assessment of myocardial fibrosis in cardiomyopathic hamsters with gadolinium-DTPA enhanced magnetic resonance imaging. Invest Radiol 1998;33(1):22-32.

22. Moon JCC, Reed E, Sheppard MN, et al. The histologic basis of late gadolinium enhancement cardiovascular magnetic resonance in hypertrophic cardiomyopathy. J Am Coll Cardiol 2004;43:2260-2264.

23. Kinney EL, Caldwell JW. Do thallium myocardial perfusion scan abnormalities predict survival in sarcoid patients without cardiac symptoms? Angiology 1990;41:573-576. 


\section{Chapter 3}

24. Yazaki Y, Isobe M, Hiroe M, et al. Prognostic determinants of long-term survival in Japanese patients with cardiac sarcoidosis treated with prednisone. Am J Cardiol 2001; 88:1006-1010.

25. Calkins H, Tandri H, Daya S. Long-term outcome of patients with cardiac sarcoidosis receiving implantable cardioverter defibrillators [abstract]. J Am Coll Cardiology 2004;43(suppl):126A. 
Table 1 Criteria for the diagnosis of cardiac sarcoidosis in this study: (modified after (4))

1. Histologic diagnosis group: endomyocardial biopsy demonstrates epithelioid granulomata without caseating granulomata.

2. Clinical diagnosis group: in patients with histologic diagnosis of extra-cardiac sarcoidosis, cardiac sarcoidosis is suspected when 'a' and at least one of criteria 'b' to'd' are present:

a. Complete RBBB, LBBB, left-axis deviation, AV-block, VT, PVC, or pathological Q or ST-T change on restingor ambulatory ECG.

b. Abnormal wall motion, regional wall thinning, or dilation of the left ventricle by echocardiography, magnetic resonance or SPECT.

c. Perfusion defect by ${ }^{20_{1}} \mathrm{Tl}$-myocardial scintigraphy.

d. Abnormal intracardiac pressure, low cardiac output, or abnormal wall motion or depressed ejection fraction of the left ventricle.

Table 2 Summary of demographic and background medical data.

\begin{tabular}{|c|c|c|c|}
\hline & $\begin{array}{l}\text { cardiac presentation } \\
\text { (group } \mathrm{A}, \mathrm{n}=19 \text { ) }\end{array}$ & $\begin{array}{l}\text { screened patients } \\
\text { (group B, } \mathrm{n}=82 \text { ) }\end{array}$ & $\mathrm{P}$ value \\
\hline Mean age (years) & $48 \pm 6$ & $45 \pm 10$ & ns \\
\hline $\begin{array}{l}\text { Gender }(\%) \\
\text { Male }\end{array}$ & $14(74 \%)$ & $55(67 \%)$ & ns \\
\hline $\begin{array}{l}\text { Race }(\%) \\
\text { Caucasian } \\
\text { African } \\
\text { Asian } \\
\text { Middle Eastern }\end{array}$ & $\begin{array}{c}17(85 \%) \\
1 \\
1 \\
0\end{array}$ & $\begin{array}{l}70(85 \%) \\
3 \\
8 \\
1\end{array}$ & ns \\
\hline $\begin{array}{l}\text { Diagnosis } \\
\text { Cardiac sarcoidosis } \\
\text { Hypertrophic cardiomyopathy } \\
\text { Coronary artery disease } \\
\text { Valvular heart disease }\end{array}$ & $\begin{array}{l}16(84 \%) \\
2 \\
2 \\
0\end{array}$ & $\begin{array}{l}3(4 \%) \\
0 \\
2 \\
1\end{array}$ & $\mathrm{P}<0.0001$ \\
\hline $\begin{array}{l}\text { Modified NYHA class } 0 \text { / I / II / III / IV } \\
\text { Time (years) since diagnosis of sarcoidosis }\end{array}$ & $\begin{array}{l}8(42 \%) / 7 / 3 / 0 / 1 \\
10 \pm 9\end{array}$ & $\begin{array}{l}66(94 \%) / 0 / 2 / 0 / 0 \\
6 \pm 8\end{array}$ & $\begin{array}{l}\mathrm{P}<0.0001 \\
\mathrm{Ns}\end{array}$ \\
\hline 0 / I/ II / III / IV & $0 / 5 / 9 / 4 / 1$ & $10 / 21 / 7 / 25 / 9$ & $\mathrm{P}<0.05$ \\
\hline Steroid treatment & $16(84 \%)$ & $47(64 \%)$ & Ns \\
\hline Hypertension & $2(11 \%)$ & $11(16 \%)$ & Ns \\
\hline Palpitations & $9(47 \%)$ & $23(34 \%)$ & Ns \\
\hline Syncope & $5(26 \%)$ & $3(9 \%)$ & Ns \\
\hline SACE maximal (IU/l) & $43 \pm 39$ & $31 \pm 23$ & Ns \\
\hline Abnormal ECG & $17(89 \%)$ & $8(10 \%)$ & $\mathrm{P}<0.0001$ \\
\hline Abnormal ambulatory ECG & $12(63 \%)$ & $7(13 \%)$ & $\mathrm{P}<0.0001$ \\
\hline Abnormal echocardiogram & $15(79 \%)$ & $7(11 \%)$ & $\mathrm{P}<0.0001$ \\
\hline Abnormal SPECT & $5(45 \%)$ & $13(26 \%)$ & Ns \\
\hline $\begin{array}{l}\text { Coronary angiography } \\
\text { Coronary artery disease }\end{array}$ & $\begin{array}{r}14 \\
2\end{array}$ & $\begin{array}{l}3 \\
0\end{array}$ & Ns \\
\hline $\begin{array}{l}\text { CMR } \\
\text { Poor image quality }\end{array}$ & $\begin{array}{c}14(74 \%) \\
0\end{array}$ & $\begin{array}{c}74(90 \%) \\
5(7 \%)\end{array}$ & \\
\hline Abnormal & $12(86 \%)$ & $12(16 \%)$ & $\mathrm{P}<0.0001$ \\
\hline Regional contrast enhancement & $9(64 \%)$ & $12(16 \%)$ & $\mathrm{P}<0.0001$ \\
\hline Decreased LVEF & $9(64 \%)$ & $1(1 \%)$ & $\mathrm{P}<0.0001$ \\
\hline Loss of wall thickness & $2(15 \%)$ & $1(1 \%)$ & ns \\
\hline Increased T2 signal & $1(5 \%)$ & 0 & ns \\
\hline
\end{tabular}




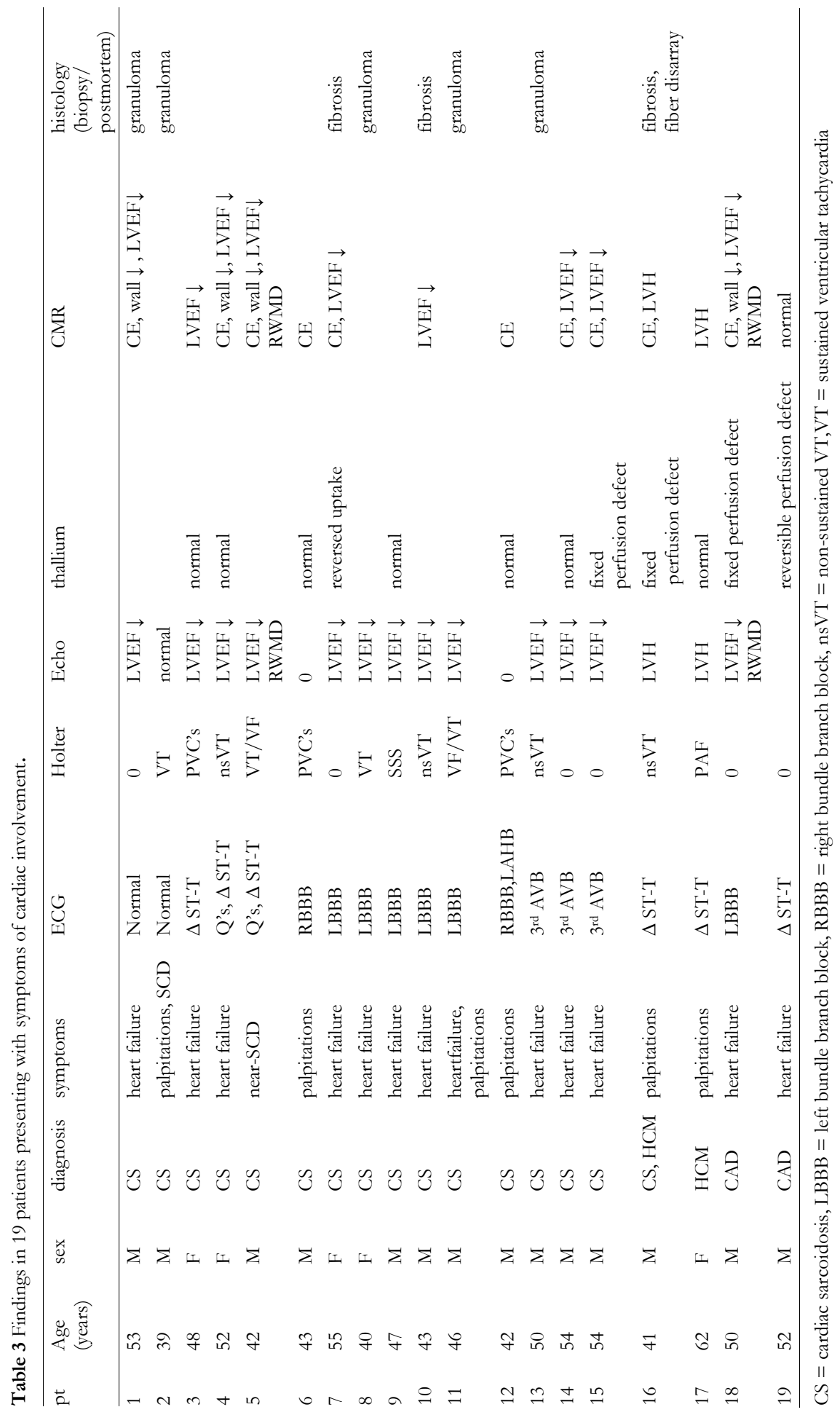


Table 4 Findings in 3 asymptomatic pulmonary sarcoidosis patients diagnosed with cardiac involvement by screening.

\begin{tabular}{lllllllll}
\hline pt & $\begin{array}{l}\text { age } \\
\text { (years })\end{array}$ & Sex & diagnosis & ECG & Holter & Echo & thallium & CMR \\
\hline 1 & 72 & F & CS & RBBB & 0 & RWMD & 0 & 0 \\
2 & 41 & M & CS & RBBB & 0 & mitral valve prolapse & fixed perfusion defect & 0 \\
3 & 49 & F & CS & $\Delta$ ST-T & 0 & 0 & reversed uptake & CE \\
\hline
\end{tabular}





\section{Chapter 4}

\section{The additional value of gadolinium enhanced magnetic resonance imaging to standard assessment for cardiac involvement in patients with pulmonary sarcoidosis}

Chest 2005;128:1629-1637.

J.P. Smedema MD, MMed(Int), G.Snoep MD, M..P.G. van Kroonenburgh, MD, PhD, R.J. van Geuns, MD, PhD, E.C. Cheriex MD, PhD, W.R.M. Dassen, PhD, A.P. M. Gorgels, $\mathrm{MD}, \mathrm{PhD}$, H.J.G.M. Crijns, MD, PhD.

Departments of Cardiology (JPS, ECC, WRMD, APMG, HJGMC), Radiology (GS), and Nuclear Medicine (MPGvK), University Hospital Maastricht, Dr Debyelaan 25, 6202 AZ, Maastricht, The Netherlands, and Department of Cardiology and Radiology, (RJvG), Erasmus Medical Centre, Rotterdam, The Netherlands. 


\section{Abstract}

Aim: To determine whether gadolinium enhanced magnetic resonance imaging (CMR) was of additional diagnostic value to standard assessment in patients with sarcoidosis who underwent evaluation for cardiac involvement.

Methods: We reviewed the findings in patients with pulmonary sarcoidosis who had been assessed with electrocardiography, Doppler-echocardiography, ${ }^{201}$ thallium scintigraphy and CMR between 2002-2004.

Results: of the 55 patients evaluated standard evaluation diagnosed cardiac involvement in 13 while CMR diagnosed myocardial scarring ( $2.5 \pm 1.9$ segments) (all 6) and impaired systolic left ventricular function (1) in an additional 6 patients. The extent of delayed enhancement correlated with disease duration $(\mathrm{p}<0.05)$, ventricular dimensions and function $(p<0.001)$, severity of mitral regurgitation $(p<0.05)$ and the presence of ventricular tachycardias $(\mathrm{p}<0.001)$. Patients in whom cardiac involvement was only diagnosed with CMR had less myocardial scarring and functional impairment $(\mathrm{p}<0.05)$ compared to patients diagnosed with standard assessment.

Conclusion: CMR provides an accurate estimation of the extent of cardiac involvement and may reveal signs of early infiltration that are not detected by standard assessment. The extent of late enhancement with gadolinium relates to severity of cardiac involvement and may therefore have prognostic implications.

\section{Contents}

Abstract 112

4.1 Introduction 113

4.2 Patients and methods 113

4.3 Results 116

4.4 Discussion 118

4.5 Conclusions 119

4.6 Acknowledgements 119

References $\quad 120$ 


\subsection{Introduction}

Sarcoidosis is a multi-system granulomatous disorder of unknown etiology with symptomatic cardiac involvement in up to $7 \%$ of patients. (1-3) Postmortem studies revealed cardiac involvement in twenty to thirty percent of patients with sarcoidosis in the United States. (4,5) In Japan cardiac involvement is even present in as many as $58 \%$ of patients, and is responsible for as many as $85 \%$ of deaths from sarcoidosis. $(\mathbf{6 , 7 )}$ The clinical features of sarcoid heart disease include congestive heart failure, cor pulmonale, supraventricular- and ventricular arrhythmias, conduction disturbances, ventricular aneurysms, pericardial effusion and sudden death. (8) The diagnosis of cardiac sarcoidosis is made by the co-existence of non-caseating granulomas on myocardial biopsy or biopsies of any extra-cardiac tissue (with the exclusion of other causes for granulomatous inflammation such as mycobacterial or fungal infection) and cardiac abnormalities for which other possible causes have been excluded. Many tests have been used to diagnose and follow up on cardiac sarcoidosis, such as standard and ambulatory electrocardiography, echocardiography, gallium- and thallium scintigraphy. (9) More recently gadolinium-enhanced cardiac magnetic resonance imaging (CMR) has been used to demonstrate myocardial inflammation, wall motion abnormalities and fibrosis in patients with cardiac sarcoidosis. (10-12) Because of superior image resolution CMR may be able to demonstrate early signs of myocardial infiltration when scintigraphy or echocardiography fail to do so. (13) The additional value of CMR to currently employed techniques, electrocardiography, echocardiography and ${ }^{201}$ thallium scintigraphy, remains to be determined.We aimed to evaluate the additional value of CMR in disclosing structural and functional cardiac abnormalities in patients with pulmonary sarcoidosis who underwent standard cardiac assessment either because of cardiac symptoms or for screening purposes, and correlated the findings with the techniques used in standard assessment.

\subsection{Patients and methods}

\section{Study population}

Between July 2002 and March 2004, 55 patients with histologically proven pulmonary sarcoidosis underwent cardiac assessment in the cardiology departments of the Erasmus Medical Centre $(n=4)$ and the University Hospital Maastricht $(n=51)$. The study group consisted of 38 men and 17 women, with a mean age of 48 and 47 respectively. Race was mainly Caucasian, 50 (91\%), 4 were Asian, 1 African.The diagnosis of pulmonary sarcoidosis was confirmed if the clinical presentation and chest radiographic findings were supported by histological evidence of non-caseating granulomas by transbronchial biopsy, and the possibility of infection, environmental factors or hypersensitivity reaction to medication causing granulomatous inflammation, had been eliminated. The patients had either presented with symptoms of cardiac involvement $(n=12)$, or were screened for cardiac involvement $(n=43)$. Patients underwent a clinical assessment, and evaluation of the maximal serum angiotensin converting enzyme levels $(n=43), 12$-lead ECG $(n=55)$, ambulatory ECG mon- 
itoring ( $\mathrm{n}=47$ ), radiological chest staging by chest X-ray and/or High Resolution CT (55), Doppler-echocardiographic evaluation $(n=55),{ }^{201}$ thallium scintigraphy (51) and gadolinium-enhanced CMR ( $\mathrm{n}=55$ ). Diagnostic coronary angiography was done in 13 patients to exclude coronary artery disease. In four patients endo-myocardial biopsies were taken. In group A (standard assessment) the diagnosis of cardiac sarcoidosis (CS) was made when endomyocardial biopsy (EMB) demonstrated non-caseating, epithelioid granulomata, or when, after the exclusion of other causes, abnormalities were present on the resting or ambulatory ECG (LBBB, RBBB, LAHB, bifascicular block, atrio-ventricular-block, VT, PVC, or pathological Q waves or ST-T changes) and echocardiogram (abnormal wall motion, regional wall thinning, or dilation of the left ventricle) or ${ }^{201}$ thallium-myocardial scintigram (irreversible perfusion defect, reversed distribution after exercise or administration of dipyridamol). In group B (CMR group) the diagnosis of cardiac sarcoidosis was based solely on abnormaltiesdetected by CMR, and the exclusion of other conditions known to cause these abnormalities such as hypertension, coronary artery or valvular heart disease. CMR findings were considered suggestive of CS when impaired systolic LV function, loss of wall thickness or gadolinium enhancing left ventricular lesions were present and other explanations had been excluded.Group C consisted of patients in whom CMR abnormalities suggestive of CS were present, but in whom standard assessment revealed no abnormalities. Because of the limited diagnostic yield of EMB, and the invasive nature with an associated risk of morbidity, we considered it not justified to systematically subject patients who were screened for CS to this procedure.

\section{Electrocardiography and ambulatory electrocardiography}

A 12-lead surface ECG was made (MAC, Marquette, Milwaukee, Wisconsin; paper speed $25 \mathrm{~mm} / \mathrm{sec}$ ) and the findings were interpreted by two experienced cardiologists and assessed for the presence of abnormalities suggestive of cardiac involvement. Ambulatory ECGs were performed for $24-72$ hours, and were considered suggestive of CS whenevidence of intermittent atrio-ventricular conduction delay or block (disregarding nightly episo-des with Wenckebach periodicity), intermittent bundle branch blocks or ventricular arrhythmias including PVCs $>100 / 24$ hours or at least 1 ventricular tachycardia (VT) was found. VT was defined as a run of PVCs containing at least 3 beats with a rate $>100$ beats per minute. Sustained VT was defined as VT with a rate $>100$ beats per minute and lasting for at least 30 seconds.

\section{Doppler-echocardiography}

Studies were performed with a Sonos 5500 phased array imaging system (Hewlett Packard, S3 transducer). Two-dimensional, continuous wave, and colour flow Doppler echocardiography was performed from standard parasternal and apical windows in long and short axes. Pulsed Doppler echocardiography was performed from an apical four-chamber view with the sample volume placed at the level of the mitral valve leaflet tips with the cursor orientated parallel to an imaginary line bisecting the left ventricle from apex to mitral valve. Left ventricular internal dimensions and wall thickness were measured at end-diastole according 
to the recommendations of the American Society of Echocardiography. (14) Left ventricular mass was calculated and indexed for body surface area. (15) Body surface area $\left(\mathrm{m}^{2}\right)$ was derived from the Dubois formula. (16) Left ventricular hypertrophy was considered present when LV mass index was $>104 \mathrm{~g} / \mathrm{m}^{2}$ for women or $>116 \mathrm{~g} / \mathrm{m}^{2}$ in men. (17) Left ventricular wall motion, thickness and thickening were determined according to the 17 segment model. (18) Thickness of the interventricular septum $>13 \mathrm{~mm}$, or wall thickness $<7 \mathrm{~mm}$ in any segment were considered abnormal. (19) Left ventricular diastolic function was evaluated by determining the iso-volumetric relaxation time, peak velocity of early $(\mathrm{E})$ and late (A) left ventricular filling, E/A ratio, deceleration rate of early diastolic flow (DT), left atrial size, and flow patterns in the pulmonary veins. Diastolic function was assessed according to the Canadian Consensus Recommendations. (20) Left ventricular ejection fraction was calculated using a modified Simpson's rule. (14) The right ventricular size and function and the presence of pericardial fluid were assessed.

\section{Thallium myocardial scintigraphy}

After treadmill peak exercise, or during intravenous infusion of dipyridamol, ${ }^{201}$ thallium was administered and SPECT performed on a Siemens triple-detector gamma camera (MultiSPECT-3), equipped with low-energy, high resolution collimators. The images were made in a $64 \times 64$ matrix ( 60 frames $/ 45$ seconds). The thallium scan was considered suggestive of CS when areas with reversed uptake and/or irreversible perfusion defects were present, and/or reversible perfusion defects were found in patients with normal coronary arteries at angiography. Regional defects were localized according to the 17 segment model (18)

\section{Cardiac Magnetic Resonance}

Studies were performed using a 1.5 Tesla MRI scanner (Philips, Best, The Netherlands or General Electric, Milwaukee, Wisconsin, USA) with a cardiac-dedicated phased-array coil. The CMR studies were ECG triggered by standard software, and obtained in diastole to minimize artifact due to cardiac motion. Studies consisted of multislice-multiphase steadystate-free precession (SSFP) in 55 patients, spin echo (SE) in 54 patients and fat saturated T2 weighted breath hold sequences in 55 patients. Studies were obtained of the short axis (SA), vertical long axis (VLA) and four chamber (4CH) views. SSFP sequences were performed to assess regional wall motion abnormalities and T2 weighted studies were performed to assess the presence of myocardial inflammation. Ten minutes after the additional administration of $0.1 \mathrm{mmol} / \mathrm{kg}$ gadolinium-DTPA (Schering, Berlin), SA and $4 \mathrm{CH}$ images were obtained with SE in 54 patients (slice thickness $8 \mathrm{~mm}$, gap $.8 \mathrm{~mm}$, matrix $512 \times 512$, FOV $380 \mathrm{~mm}$, voxel size $0.7 \mathrm{~mm} \times 0.7 \mathrm{~mm} \times 8 \mathrm{~mm})$ and 3-D breath hold inversion recovery-gradient echo (IR-GRE) sequences (SA, VLA, $4 \mathrm{CH}$ ) in 15 patients (slice thickness $10 \mathrm{~mm}$, no gap, matrix $256 \times 256$, FOV $400 \mathrm{~mm}$, voxel size $1.6 \mathrm{~mm} \times 1.6 \mathrm{~mm} \times 10 \mathrm{~mm}$ ) to assess for the presence of gadolinium enhancing enhancing lesions (LGE). Fourteen patients had both SE and IR-GRE studies. The inversion time (250-400 $\mathrm{msec}$ ) was determined on an individual base to obtain optimal nulling of the un-enhanced myocardial sig- 
nal. Regional differences in left ventricular wall enhancement were measured and localized according to the 17 segment model (18) The total time required for the investigation was 45-60 minutes. The studies were independently evaluated by 4 blinded observers, 3 cardiologists and 1 radiologist, all experienced in performing CMR. The studies were considered to be abnormal when at least 2 observers described identical abnormalities.

\section{Coronary angiography}

Diagnostic coronary angiography $(\mathrm{n}=13)$ was performed when considered indicated by the managing physician, and was generally done in patients with symptoms and findings suggestive of coronary artery disease.

\section{Statistical analysis}

All statistical analyses were performed using the statistical software package SSPS version 11.5. Group data are expressed as mean \pm SD. Continuous variables were assessed using the Student unpaired $t$ test for independent samples or the Mann Whitney test were appropriate, and all categorical variables were assessed using the chi-square test. Statistical significance was defined as a p value less than 0.05 .

\subsection{Results}

\section{Findings at standard evaluation}

Standard assessment diagnosed cardiac sarcoidosis in 13 patients. The demographic and clinical characteristics of the patients are presented in Table 1. During a mean follow up of $19 \pm 7$ months, of the patients diagnosed with CS with standard assessment 1 patient died suddenly, 2 received a DDD-R pacemaker because of $3^{\text {rd }}$ degree atrio-ventricular block, and 4 received a pacemaker/implantable cardioverter defibrillator of ventricular tachyarrhythmias. The follow-up of patients diagnosed with CS solely because of CMR abnormalities (group C) was uneventful.

\section{Findings with CMR}

The findings with CMR are presented in Table 1. In $37(67 \%)$ studies 4 out of 4 observers, in $12(22 \%)$ studies 3 out of 4 observers, and in $6(11 \%)$ studies 2 out of 4 observers agreed on the absence or the presence and localization of late enhancement. Late gadolinium enhancing lesions (LGE) were present in 17. Seven of 17 patients had evaluation with SE, 9 with SE and IR-GRE, and 1 patient had only IR-GRE. In 6 of the SE patients 2, and in 1 of the SE patients 3 observers agreed on the presence and localization of LGE, while in 4 of the 10 patients who had both SE and IR-GRE or only IR-GRE 3 observers, and in the remaining 6 all observers agreed on the presence and localization of LGE. 
In 19 patients (group B), including all patients diagnosed by standard assessment (group A), the CMR examinations showed abnormalities that were suggestive of cardiac sarcoidosis. In 6 patients late enhancement, involving 1 - 6 segments (mean $2.5 \pm 1.9$ ), one of whom also had impaired systolic left ventricular (LV) function (LVEF 52\%) was diagnosed by CMR, while standard assessment was normal. (Figures 3 and 4) The amount of myocardial enhancement in group A did not significantly differ from group C. The transmural extent of enhancement and number of enhancing segments both showed a negative correlation with $L V$ function $(p<0.05)$. The presence of late enhancement correlated with a primary cardiac presentation $(p<0.001)$, and the duration of disease $(p<0.05)$. The presence of myocardial enhancement did not correlate with the extent of pulmonary disease. The bulls eye representation reflecting the distribution of LV enhancement is shown in Figure 1. Late enhancement predominantly involved the basal segments of the left ventricle $(59 \%)$, with the anteroseptal and anterolateral segments most affected (43\%). (Figures 5, 6 and 7 )

Correlation of standard evaluation with the CMR findings

Figures 2A and $\mathbf{B}$ illustrate the correlations between the different investigations and CMR.

\section{Electrocardiography}

The findings with the resting ECGs are presented in Table 2. The presence of regional wall motion abnormalities ( $p<0.001)$, impaired LV function $((p<0.001)$ and the segmental extent $(p<0.05)$ of late enhancement all correlated with the ECG abnormalities. The transmural extent and distribution of late enhancement did not determine the presence of conduction abnormalities on the ECG. Transmural enhancement $(p<0.001)$ and segmental extent of enhancement $(\mathrm{p}<0.05)$ correlated with the presence of $\mathrm{Q}$ waves. The presence of impaired LV function $(p<0.05)$ and regional wall motion abnormalities $(p<$ 0.001) correlated with the abnormalities found with ambulatory ECG mentioned in Table 2 . The extent of segmental enhancement $(p<0.001)$ and transmural enhancement correlated with the presence of (non)sustained ventricular arrhythmias.

\section{Doppler Echocardiography}

The findings with Doppler echocardiography are presented in Table 3. The segmental and transmural extent of enhancement correlated with regional wall motion abnormalities $(\mathrm{p}<$ $0.05)$, mitral regurgitation $(\mathrm{p}<0.001)$, LV dimensions $(\mathrm{p}<0.05)$, and impaired diastolic and systolic left and systolic right ventricular function $(\mathrm{p}<0.05)$. 


\section{Thallium scintigraphy}

${ }^{201}$ Thallium scintigraphy demonstrated reversed distribution or irreversible perfusion defects in 6 patients. The presence of perfusion defects correlated with late enhancement and impaired systolic LV function $(\mathrm{p}<0.05)$. The localization of perfusion defects and late enhancement matched in 4 of the 6 patients. In 1 patient reversed distribution was present in the apex, while ECG, echocardiography and CMR were normal.

\subsection{Discussion}

The diagnostic potential of CMR for detecting cardiac sarcoidosis has been demonstrated by several case-reports and single-centre patient series. (10-12,21-28) Patel et al assessed 58, mainly african american, sarcoidosis patients without cardiac symptoms and reported a two-fold higher rate of cardiac involvement with gadolinium-enhanced CMR compared to evaluation with ECG and echocardiography. (28)Sköld et al. recently reported on 18 subsequent patients with pulmonary sarcoidosis who had had been systematically evaluated for cardiac involvement with ECG, Doppler-echocardiography and CMR. (29) Our study is the first to evaluate the value of comprehensive CMR assessment in addition to resting and ambulatory ECGs, Doppler-echocardiography and ${ }^{201}$ thallium scintigraphy. We found CMR to be highly sensitive in detecting abnormalities that suggest cardiac involvement in patients with histologically confirmed pulmonary sarcoidosis. Our findings demonstrate a strong correlation between electrocardiographic and echocardio- graphic abnormalities and the amount of left ventricular enhancement with CMR. The distribution of enhancement correlates well with the localization of inflammation and fibrosis reported in postmortem studies of patients with CS. $(4,5)$ Limited, non-transmural or patchy myocardial scar tissue, frequently found at postmortem evaluation of CS patients, may remain undetected by electrocardiography, ultrasound or scintigraphy, but be detected by CMR due to its high image resolution and inversion-recovery gradient echo sequences that increase signal intensity differences by almost 500\%. $(\mathbf{1 3 , 3 0 )}$ The additional value of CMR to standard assessment was demonstrated in 6 patients $(11 \%)$ in whom left ventricular involvement was only diagnosed by CMR. Since disease in this group was of shorter duration in comparison to the group diagnosed with standard assessment, and myocardial involvement limited, the findings in these patients may well reflect early involvement. The follow up of this group, though uneventful, may be to short ( $21 \pm 7$ months) to draw firm conclusions concerning the prognostic relevance of the CMR findings. Electrocardiography is considered an appropriate screening test for cardiac sarcoidosis, however, in $25 \%$ of patients with gross cardiac infiltration at postmortem assessment ECG abnormalities had not been present during life. $(4,31,32)$ Ambulatory electrocardiography is reported to have a sensitivity of $67 \%$ and specificity of $80 \%$ for the diagnosis of cardiac sarcoidosis. (33) Abnormalities on 2-D echocardiograms, increase or loss of wall thickness, ventricular dilation, functional impairment, mitral regurgitation, or the presence of pericardial effusions, have been detected in $14-41 \%$ of patients with sarcoidosis, even in the absence of ECG abnormalities and clinical symptoms. (34-39) Impaired diastolic function was found in $14-56 \%$ of patients. 
$(27,37)$ In our study population unexplained diastolic relaxation abnormalities were present in $19(35 \%)$ patients without evidence of cardiac involvement. CMR did not reveal scar tissue in this group and, unlike a previous study, its presence did not correlate with disease duration. $(29,39){ }^{201}$ Thallium scintigraphy has been most extensively studied and revealed perfusion defects diagnostic of myocardial fibrosis or granulomatous infiltration in 13 $32 \%$ of sarcoidosis patients. $(\mathbf{4 0 , 4 1 )}$. Both electrocardiography and echocardiography have demonstrated prognostic value in patients with CS. The presence of sustained ventricular tachycardias, NYHA functional class and left ventricular dilation were found to be strong independent predictors of mortality. (42) The correlation between the presence of nonsustained and sustained VTs on Holter and the extent of left ventricular enhancement with CMR in sarcoidosis has not been reported before. Myocardial scar tissue is considered to be the substrate for ventricular tachyarrhythmia's due to electrical reentry. (43) In our study population the presence of VTs did not correlate with substrate in a particular myocardial layer, since delayed enhancement involved parts of all three myocardial layers in most patients. Further study is needed to elucidate the diagnostic and prognostic relevance of CMR in the management of this condition.

\subsection{Conclusions}

CMR is a useful technique for the assessment of cardiac involvement in sarcoidosis, since the findings correlated strongly with standard assessment, and the diagnosis of CS according to accepted guidelines. CMR may be able to demonstrate disease at an early stage, when standard assessment fails to do so. In addition, extent of late enhancement with gadolinium correlated with ventricular dilatation, functional impairment, and the presence of ventricular arrhythmias, all known markers of sudden death. CMR based early detection and monitoring of cardiac sarcoidosis may represent a useful strategy to determine optimal timing of medical intervention.

\subsection{Acknowledgements}

The authors gratefully acknowledge H.Kuehl, MD, $\mathrm{PhD}$, Aachen University Medical Centre, and A.M.Beek, MD, Free University Medical Centre, Amsterdam, for assessing the CMR studies. We thank J.H..M.Schreur, MD, PhD, Medical Centre Haaglanden, The Hague, for allowing us to include his patient in our study, and G Zonneveld for expert assistance in preparing the figures. 


\section{References}

1. Johns CJ, Michele TM. The clinical management of sarcoidosis: a 50-year experience at the Johns Hopkins Hospital. Medicine 1999;78:65-111.

2. Shammas RL, Movahed A. Sarcoidosis of the heart. Clin Cardiol 1993;16:462-72.

3. Hagemann GJ, Wurm K. The clinical, electrocardiographic and pathological features of cardiac sarcoid. In: Jones Williams W, Davies BH, eds. Sarcoidosis and other granulomatous disorders. Proceedings of the 8th International Conference. Cardiff: Alfa Omega Publishing, 1980:601-6.

4. Silverman KJ, Hutchins GM, Bulkley BH. Cardiac sarcoid: a clinico-pathologic study of 84 unselected patients with systemic sarcoidosis. Circulation 1979;58:1204-11.

5. Roberts WC, McAllister HA Jr, Ferrans VJ. Sarcoidosis of the heart: a clinico-pathological study of 35 necropsy patients (group 1) and review of 78 previously described

6. Matsui Y, Iwai K, Tachibana T, et al. Clinico-pathological study on fatal myocardial sarcoidosis. Ann NY Acad Sci 1976;278:455-69.

7. Tachibana T, Iwai K, Takemura T. Study on the cause of death in patients with sarcoidosis in Japan. XII World Congress on Sarcoidosis, Kyoto, Japan, September 8-13,1991. Abstract.8. Sharma OP, Maheshwari, Thaker K. Myocardial sarcoidosis. Chest 1993; 103:253-7.

9. Deng JC, Baughman RP, Lynch JP. Cardiac involvement in sarcoidosis. Sem Resp Crit Care Medicine 2002;23:513-26.

10. Shimada T, Shimada K, Sakane T, et al. Diagnosis of cardiac sarcoidosis and evaluation of the effects of steroid therapy by gadolinium-DTPA-enhanced magnetic resonance imaging. Am J Med 2001;110:520-7.

11. Vignaux O, Dhôte R, Duboc D, et al. Detection of myocardial involvement in patients with sarcoidosis applying T2-weighted, contrast-enhanced and cine magnetic resonance imaging: initial results of a prospective study. J Comput Assist Tomograph 2002;26:762-7.

12. Vignaux $\mathrm{O}$, Dhôte $\mathrm{R}$, Duboc $\mathrm{D}$, et al. Clinical significance of myocardial magnetic resonance abnormalities in patients with sarcoidosis. A 1-year follow-up study. Chest 2002;122:1895-1901.

13. Wagner A, Mahrholdt H, Holly TA, et al. Contrast-enhanced MRI and single photon emission computed tomography (SPECT) perfusion imaging for the detection of subendocardial myocardial infarcts: an imaging study. Lancet 2003;361:374-379.

14. Schiller NB, Shah PM, Crawford M, et al. American Society of Echocadiography Committee on Standards, Subcommittee on Quantification of Two-Dimensional Echocardiograms: recommendations for quantification of the left ventricle by two-dimensional echocardiography. J Am Soc Echocardiography 1989;2:358-67.

15. Devereux RB, Alonso DR, Lutas EM, et al. Echocardiographic assessment of left ventricular hypertrophy: comparison to necropsy findings. Am J Cardiol 1986;57:450-8.

16. Du Bois D, Du Bois E. A formula to estimate the approximate surface area if height and weight be known. Nutrition 1989;5:303-11.

17. Okin PM, Devereux RB, Nieminen MS, et al. Relationship of the electrocardiographic strain pattern to left ventricular structure and function in hypertensive patients: The LIFE study. J Am Coll Cardiol 2001;38:51420.

18. AHA writing group on myocardial segmentation and registration for cardiac imaging. Standardized myocardial segmentation and nomenclature for tomographic imaging of the heart. Circulation 2002;105:539-42.

19. Yazaki Y, Isobe M, Hiramitsu S, et al. Comparison of clinical features and prognosis of cardiac sarcoidosis and idiopathic dilated cardiomyopathy. Am J Cardiol 1998;82:537-40.

20. Rakowski H, Appleton CP, Chan K-L, et al. Recommendations for the measurement and reporting of diastolic function by echocardiography. J Am Soc Echocardiography 1996;9:736-60.

21. Doherty MJ, Kumar SK, Nicholson AA, et al. Cardiac sarcoidosis: the value of magnetic resonance imaging in diagnosis and assessment of response to treatment. Resp Med 1998;92:697-99.

22. Schulz-Menger J, Strohm O, Dietz R, et al. Visualization of cardiac involvement in patients with systemic sarcoidosis applying contrast-enhanced magnetic resonance imaging. MAGMA 2000;82-3.

23. Chandra M, Silverman ME, Oshinski J, et al. Diagnosis of cardiac sarcoidosis aided by MRI. Chest 1996;110:562-5.

24. Inoue SI, Shimada T, Murakami Y. Clinical significance of gadolinium-DTPA enhanced MRI for detection of myocardial lesion in patients with sarcoidosis. Clin Radiol 1999;54:70-72.

25. Matsuki M, Matsuo M. MR findings of myocardial sarcoidosis. Clin Radiol 2000;55:323-5. 
26. Dhôte R, Vignaux $\mathrm{O}$, Blanche $\mathrm{P}$, et al. Value of MRI for the diagnosis of cardiac involvement in sarcoidosis. Revue Med Int 2003;24:151-7.

27. Vignaux O. Cardiac sarcoidosis: spectrum of MRI features. AJR 2005;184:249-54.

28. Patel MR, Cawley PJ, Heitner JF, et al. Delayed enhanced MRI improves the ability to detect cardiac involvement in patients with sarcoidosis [abstract]. Circulation 2004;110(suppl):2995.

29. Skold CM, Larssen FF, Rasmussen E, et al. Detemination of cardiac involvement in sarcoidosis by MRI and Doppler echocardiography. J Int Med 2002;252:465-71.

30. Simonetti OP, Kim RJ, Fieno DS, et al. An improved MR imaging technique for the visualzation of myocardial infarction. Radiology 2001;218:215-223.

31. Numao Y, Sekiguchi M, Fruie T, et al. A study of cardiac involvement in 963 cases of sarcoidosis by ECG and endomyocardial biopsy. Ann NY Acad Sci 1976;76:607-14.

32. Thunell M, Bjerle P, Stjernberg N. ECG abnormalities in patients with sarcoidosis. Acta Med Scand 1983;213:115-8.

33. Suzuli T, Kanda T, Kubota S, et al. Holter monitoring as a non-invasive indicator of cardiac involvement in sarcoidosis. Chest 1994;106:1021-4.

34. Lewin RF, Mor R, Spitzer S, et al. Echocardiographc evaluation of patients with systemic sarcoidosis. Am Heart J 1985;110:116-22.

35. Burstow DJ, Tajik J, Baily KR, et al. Two-dimensional echocardiographic findings in systemic sarcoidosis. Am J Cardiol 1989;63:478-82.

36. Gibbons WJ, Levy RD, Nava S, et al. Subclinical cardiac dysfunction in sarcoidosis. Chest 1991;100:44-50.

37. Kinney EL, Jackson GL, Reeves WC, et al. Thallium-scan myocardial defects and echocardiographic abnormalities in patients with sarcoidosis without clinical cardiac dysfunction. Am J Med 1980;68:497-503.

38. Angomachelelis N, Hourzamanis A, Vamvalis C, et al. Doppler echocardiographic evaluation of left ventricular diastolic function in patients with systemic sarcoidosis. Postgrad Med J 1992;68(suppl 1):S52-S56.

39. Fahy GJ, Warwick T, McCreery CJ, et al. Doppler echocardiographic detection of left ventricular diastolic dysfunction in patients with pulmonary sarcoidosis. Chest 1996;109:62-66.

40. Le Gudulec D, Menad F, Fraggi M, et al. Myocardial sarcoidosis. Clinical value of technetium- $99 \mathrm{~m}$ sestamibi tomoscintigraphy. Chest 1994;106:1675-82.

41. Haywood LJ, Sharma OP, Siegel ME, et al. Detection of myocardial sarcoidosis by thallium 201 imaging. J Natl Med Assoc 1982;74:959-64.

42. Yazaki Y, Isobe M, Hiroe M, et al. Prognostic determinants of long-term survival in Japanese patients with cardiac sarcoidosis treated with prednisone. Am J Cardiol 2001;88:1006-10.

43. Furushima $\mathrm{H}$, Chinushi M, Sugiura $\mathrm{H}$, et al. Ventricular tachyarrhythmia associated with cardiac sarcoidosis its mechanisms and outcome. Clin Cardiol 2004;27:217-22. 
Table 1: Summary of the clinical and demographic patients characteristics and findings with CMR.

\begin{tabular}{|c|c|c|c|}
\hline Abnormal CMR studies & $\begin{array}{l}\text { cardiac sarcoidosis } \\
\text { standard assessment } \\
\text { (group } \mathrm{A}, \mathrm{n}=13 \text { ) }\end{array}$ & $\begin{array}{l}\text { cardiac sarcoidosis } \\
\text { only with } \mathrm{CMR} \\
\text { (group } \mathrm{C}, \mathrm{n}=6 \text { ) }\end{array}$ & $\mathrm{P}$ value \\
\hline Mean age (years) & $49 \pm 6$ & $50 \pm 10$ & ns \\
\hline Gender $(\%)$ male/female & $9(69 \%) / 4$ & $4(67 \%) / 2$ & ns \\
\hline Time (years) since diagnosis & $9 \pm 8$ & $5 \pm 2$ & ns \\
\hline Follow up (months) since cardiac assessment & $18 \pm 10$ & $21 \pm 7$ & ns \\
\hline $\begin{array}{l}\text { Modified NYHA class } 0 \text { (no complaints) } \\
\text { / I / II / III / IV }\end{array}$ & $7 / 5 / 0 / 0 / 1$ & $6 / 0 / 0 / 0 / 0$ & ns \\
\hline Pulmonary stage 0/ I / II / III / IV & $0 / 3 / 4 / 6 / 0$ & $1 / 3 / 0 / 1 / 1$ & ns \\
\hline Palpitations & $4(31 \%)$ & $4(67 \%)$ & ns \\
\hline Maximal serum ACE (IU/l) mean \pm SD & $49 \pm 40$ & $31 \pm 12$ & ns \\
\hline $\mathrm{LVEF}(\%)$ mean $\pm \mathrm{SD}$ & $45 \pm 17$ & $60 \pm 6$ & $?$ \\
\hline $\begin{array}{l}\text { Number of patients with late enhancement } \\
\text { Number of segments per patient }\end{array}$ & 11 & 6 & \\
\hline $\begin{array}{l}\text { Range } \\
\text { Mean } \pm \text { SD }\end{array}$ & $\begin{array}{l}0-12 \\
4.1 \pm 4.2\end{array}$ & $\begin{array}{l}1-6 \\
2.5 \pm 1.9\end{array}$ & ns \\
\hline $\begin{array}{l}\text { Localization of gadolinium enhancement (\%): } \\
\text { Basal segments (1-6) } \\
\text { Equatorial segments (7-12) } \\
\text { Apical segments (13-16) }\end{array}$ & $\begin{array}{c}30(57 \%) \\
19(36 \%) \\
4(7 \%)\end{array}$ & $\begin{array}{l}10(67 \%) \\
5(33 \%) \\
0\end{array}$ & $\begin{array}{l}\mathrm{ns} \\
\mathrm{ns} \\
\mathrm{ns}\end{array}$ \\
\hline $\begin{array}{l}\text { Transmural distribution of gadolinium enhanc } \\
\text { Subendocardial layer (\%) } \\
\text { Middle layer (\%) } \\
\text { Subepicardial layer (\%) } \\
\text { All three layers (\%) } \\
\text { Confluent transmural (\%) }\end{array}$ & $\begin{array}{l}9(82 \%) \\
9(82 \%) \\
9(82 \%) \\
9(82 \%) \\
3(27 \%)\end{array}$ & $\begin{array}{l}6(100 \%) \\
5(67 \%) \\
2(33 \%) \\
2(33 \%) \\
0\end{array}$ & $\begin{array}{l}\text { ns } \\
\text { ns } \\
\text { ns } \\
\text { ns } \\
\text { ns }\end{array}$ \\
\hline Regional wall motion abnormalities & 7 & 0 & $\mathrm{P}<0.05$ \\
\hline Loss of wall thickness $<7 \mathrm{~mm}$ & 2 & 0 & ns \\
\hline
\end{tabular}

Table 2: Summary of electrocardiographic findings.

\begin{tabular}{llll}
\hline Findings with 12-lead electrocardiogram & $\begin{array}{l}\text { cardiac sarcoidosis } \\
\text { standard assessment } \\
\text { (group A, n = 13) }\end{array}$ & $\begin{array}{l}\text { cardiac sarcoidosis } \\
\text { only with CMR } \\
\text { (group C, n = 6) }\end{array}$ & P value \\
\hline Normal & $1(7 \%)$ & $6(100 \%)$ & $\mathrm{P}<0.001$ \\
Abnormal & 12 & 0 \\
LBBB & 2 & 0 \\
RBBB & 1 & 0 \\
Bifascicular block & 1 & 0 \\
LAHB & 1 & 0 \\
Grade III atrio-ventricular block & 2 & 0 \\
Pseudo-infarct pattern & 2 & 0 \\
ST-T segment abnormalities & 3 & 0 \\
Findings with ambulatory electrocardiography & & \\
Abnormal & $6(46 \%)$ & 0 \\
PVC $>$ 100/24 hrs & 2 & 0 \\
Non-sustained VT & 3 & 0 \\
Sustained VT & 1 & 0 \\
Paroxysmal atrial fibrillation & 0 & 0 \\
Atrioventricular nodal reentrant tachycardia & 0 & 0 \\
\hline
\end{tabular}


Table 3: Summary of echocardiographic findings.

\begin{tabular}{|c|c|c|c|}
\hline Echocardiographic findings & $\begin{array}{l}\text { cardiac sarcoidosis } \\
\text { standard assessment } \\
\text { (group } \mathrm{A}, \mathrm{n}=13 \text { ) }\end{array}$ & $\begin{array}{l}\text { cardiac sarcoidosis } \\
\text { only with } \mathrm{CMR} \\
\text { (group } \mathrm{C}, \mathrm{n}=6 \text { ) }\end{array}$ & $\mathrm{P}$ value \\
\hline \multicolumn{4}{|l|}{ Diastolic dysfunction } \\
\hline Relaxation abnormalities & 2 & 1 & ns \\
\hline Pseudonormalization & 1 & 1 & ns \\
\hline Restrictive filling pattern & 3 & 0 & ns \\
\hline $\mathrm{LVH}$ & 1 & 1 & ns \\
\hline \multicolumn{4}{|l|}{ LVEF (\%) } \\
\hline Mean $\pm S D$ & $47 \pm 16$ & $62 \pm 4$ & $\mathrm{P}<0.05$ \\
\hline $\mathrm{LVEF} \leq 55 \%$ & 8 & 0 & $\mathrm{P}<0.05$ \\
\hline Regional wall motion abnormalities & 7 & 0 & $\mathrm{P}<0.05$ \\
\hline Regional wall thinning & 2 & 0 & ns \\
\hline Mitral regurgitation & & & ns \\
\hline Grade 1 & 1 & 0 & \\
\hline Grade 2 & 3 & 0 & \\
\hline Grade 3 & 0 & 0 & \\
\hline Grade 4 & 2 & 0 & \\
\hline Tricuspid regurgitation & 1 & 0 & ns \\
\hline Pulmonary hypertension & 2 & 1 & ns \\
\hline Range $(\mathrm{mm} \mathrm{Hg})$ & $30-55$ & 30 & \\
\hline Right ventricular hypertrophy & 3 & 1 & ns \\
\hline Right ventricular dysfunction & 6 & 0 & $\mathrm{P}<0.05$ \\
\hline Pericardial effusion & 0 & 0 & ns \\
\hline
\end{tabular}
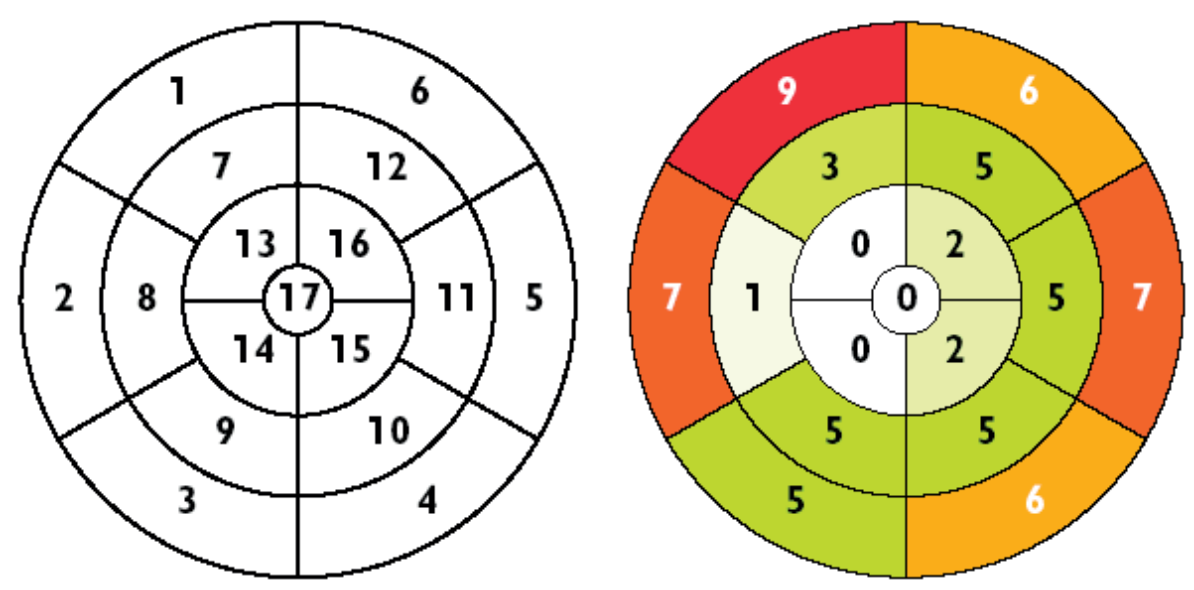

Figure 1. Bulls eye plots demonstrating left ventricular segmentation (left) (18) and the distribution of late enhancement per segment in 19 sarcoidosis patients (right). 
Chapter 4

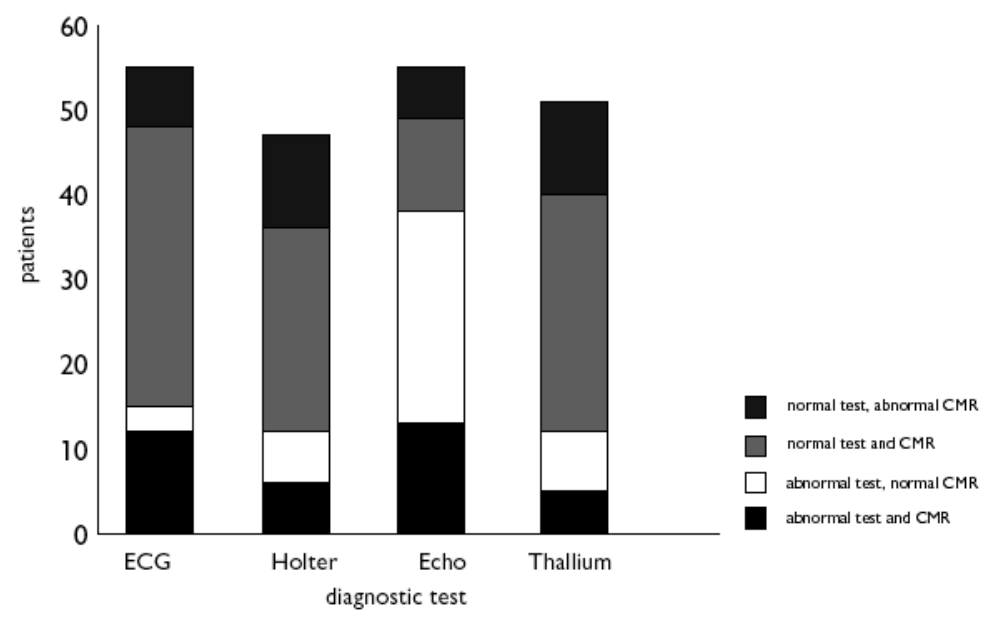

Figure 2A. Bar diagram showing the distribution of abnormal test results and correlation with late enhancement at magnetic resonance imaging.

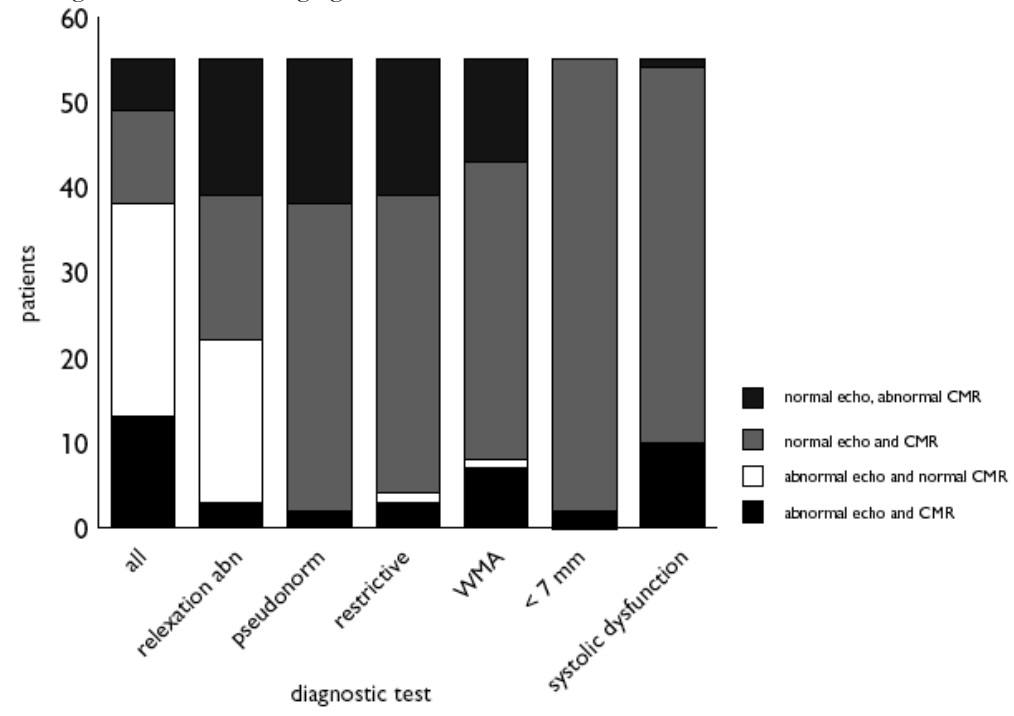

Figure 2B. Bar diagram showing the distribution of abnormal findings at Doppler echocardiography and correlation with late enhancement at magnetic resonance imaging. 


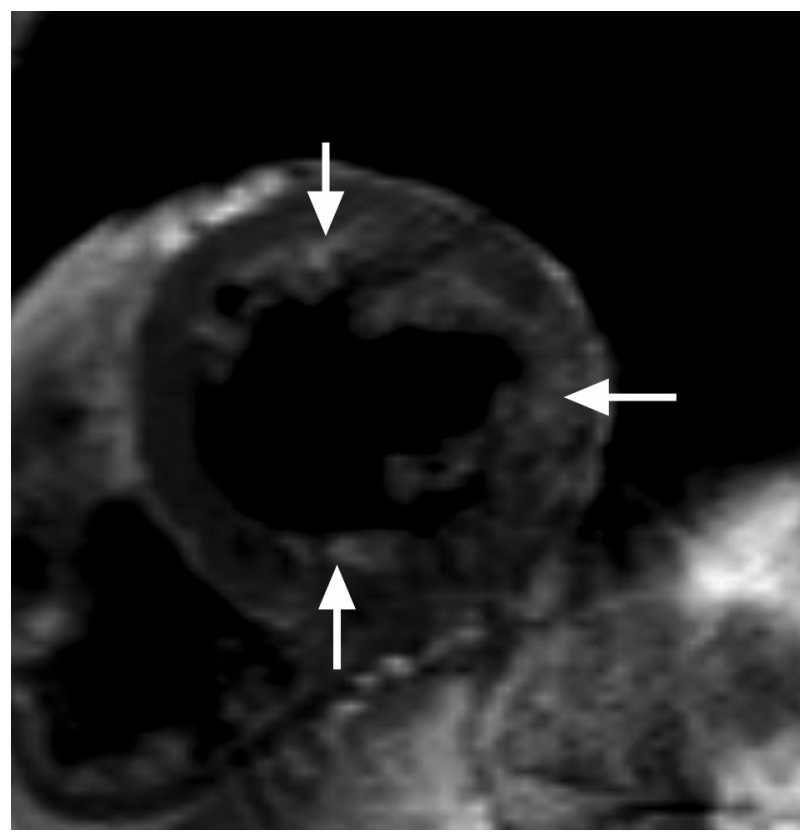

Figure 3. CMR study (spin echo (SE) sequence, short axis view (SA)) demonstrates late enhancement (segments 1,4 and 5) in a patient in whom standard assessment had not revealed abnormalities.

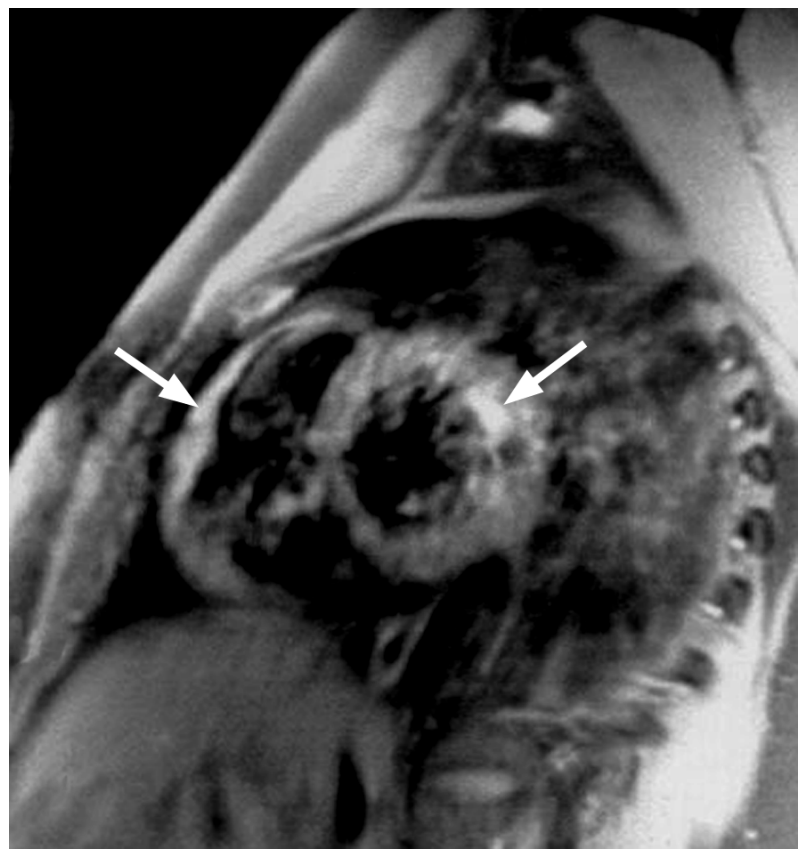

Figure 4. CMR study (spin echo (SE) sequence, short axis view (SA)) demonstrates late enhancement (segments 7 and 12) in a patient in whom standard assessment had initially not revealed abnormalities. 

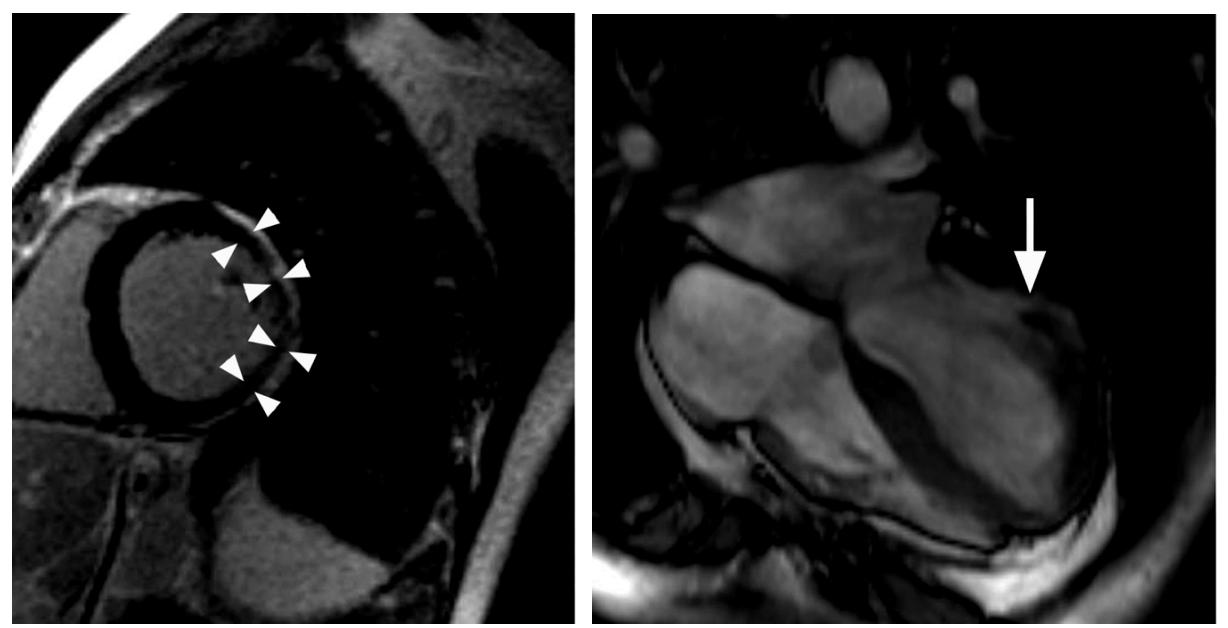

Figure 5. CMR study (left: $3 \mathrm{D}$ breath-hold inversion recovery fast gradient echo IR-FGRE, short axis view; right: SSFP, 4 chamber view) demonstrates loss of wall thickness and late enhancement (segments 11 and 12) in a patient who survived out of hospital cardiac arrest secondary to ventricular fibrillation.

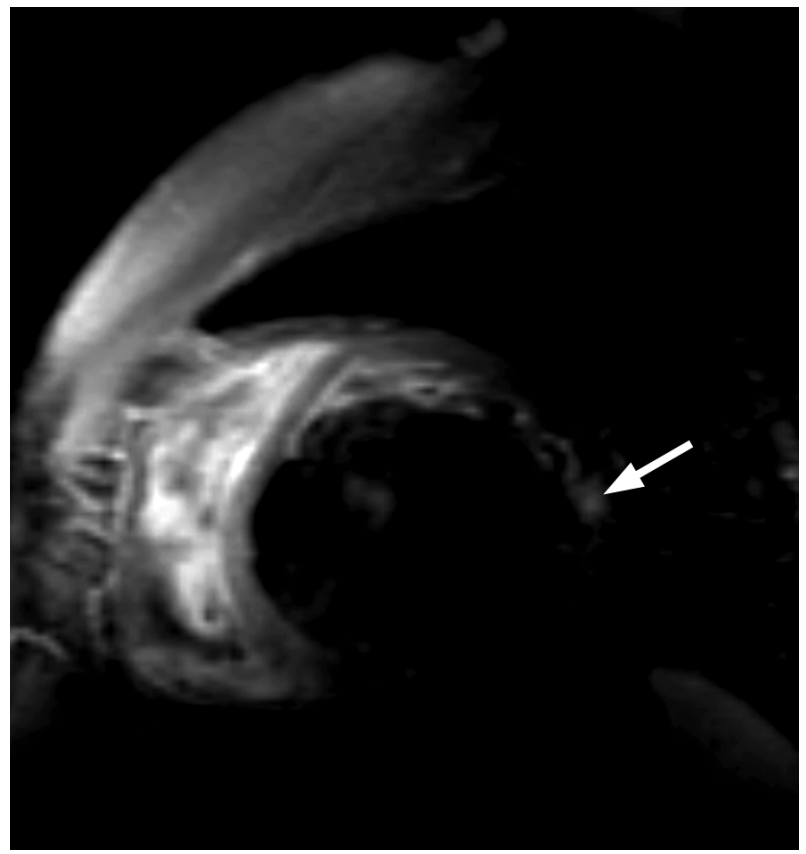

Figure 6. CMR study (spin echo (SE) sequence, short axis (SA) view) demonstrates left ventricular dilation and late enhancement (segment 6 ) in a patient who presented with heart failure and left bundle branch block. 
The additional value of gadolinium enhanced magnetic resonance imaging
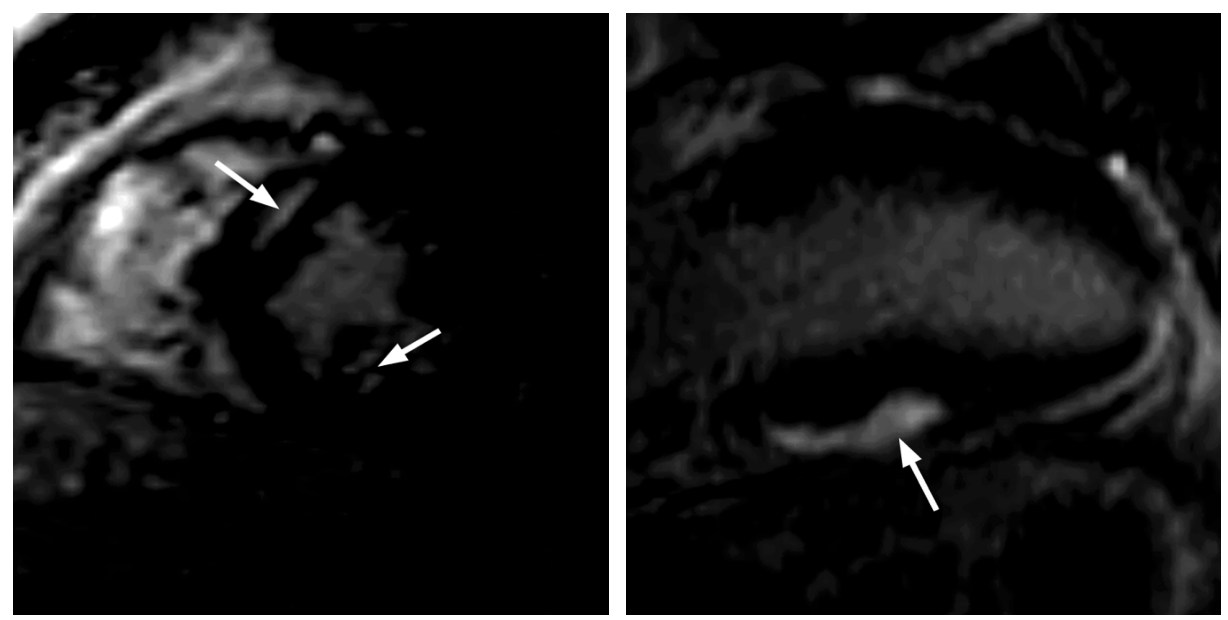

Figure 7. CMR study (3 D breath-hold inversion recovery fast gradient echo (IR-FGRE), left: short axis (SA), and right: vertical long axis (VLA) views) demonstrates late enhancement (segments $2,8,10,12$ ) in a patient who presented with sustained monomorphic VT, originating from the inferolateral left ventricular wall. 



\section{Chapter 5}

\section{Evaluation of the accuracy of gadolinium- enhanced cardiovascular magnetic resonance in the diagnosis of cardiac sarcoidosis}

J Am Coll Cardiol 2005;45(10):1683-1690.

J.P. Smedema, MD, MMed(Int), FCP(SA), G. Snoep, MD, M.P.G. van Kroonenburgh, $\mathrm{MD}, \mathrm{PhD}$, R.J. van Geuns, MD, PhD, W.R.M. Dassen, PhD, A.P.M. Gorgels, MD, PhD, FACC, H.J.G.M. Crijns, MD, PhD, FESC.

Departments of Cardiology (JPS, WRMD, HJGMC, APG), Radiology (GS), Nuclear Medicine (MPGvK), University Hospital Maastricht, Dr Debyelaan 25, 6202 AZ, Maastricht, Department of Cardiology and Radiology (RJvG), Erasmus Medical Centre, Rotterdam. 


\title{
Structured abstract
}

Objectives: This study analyzed the accuracy of gadolinium-enhanced cardiovascular magnetic resonance (CMR) for the diagnosis of cardiac sarcoidosis (CS).

Background: The diagnosis of CS is made according to the guidelines of the Japanese Ministry of Health and Welfare (1993). CMR has not been incorporated in the guidelines, and the diagnostic accuracy of CMR for the diagnosis of CS has not yet been evaluated.

Methods: We performed an analysis of the 12-lead ECGs, 24-hours ambulatory ECGs, echocardiograms, thallium scintigrams and gadolinium-enhanced CMR studies in 58 biopsy proven pulmonary sarcoidosis patients assessed for CS. The diagnostic accuracy of CMR for CS was determined with modified Japanese guidelines as gold standard.

Results: The diagnosis of CS was made in 12 of 58 patients (21\%). CMR revealed late gadolinium enhancement (LGE), mostly involving basal and lateral segments (73\%), in 19 patients. In 8 of the 19 patients scintigraphy was normal, while patchy LGE was present. The sensitivity and specificity of CMR were 100\% (95\% CI, 78-100\%) and 78\% (95\% CI, $64-89 \%$ ) the PPV and NPV 55\% respectively $100 \%$, with an overall accuracy of $83 \%$.

Conclusions: In patients with sarcoidosis CMR is a useful diagnostic tool to determine cardiac involvement. New diagnostic guidelines should include CMR.

\section{Contents}

$\begin{array}{lr}\text { Structured abstract } & 130\end{array}$

Abbreviation list $\quad 130$

$\begin{array}{lll}5.1 & \text { Introduction } & 131\end{array}$

5.2 Patients and methods 131

$\begin{array}{lll}5.3 & \text { Results } & 133\end{array}$

$\begin{array}{lll}5.4 & \text { Discussion } & 134\end{array}$

$\begin{array}{lll}5.5 & \text { Conclusions } & 135\end{array}$

5.6 Study limitations 136

5.7 Recommendations for further study 136

5.8 Acknowledgements 136

$\begin{array}{ll}\text { References } & 137\end{array}$

\author{
Abbreviation list \\ ECG electrocardiogram \\ CMR Cardiovascular Magnetic Resonance \\ CS cardiac sarcoidosis \\ LGE late gadolinium enhancement \\ VT ventricular tachycardia
}




\subsection{Introduction}

Sarcoidosis is a multi-system granulomatous disorder of unknown etiology with symptomatic cardiac involvement in up to seven percent of patients. (1-3) Postmortem studies reveal cardiac involvement in twenty to thirty percent of patients with sarcoidosis in the United States. $(4,5)$ In Japan cardiac involvement is present in as many as $58 \%$ of patients, and is responsible for as many as $85 \%$ of deaths from sarcoidosis. $(6,7)$ The clinical features of sarcoid heart disease include congestive heart failure, cor pulmonale, supraventricular- and ventricular arrhythmias, conduction disturbances, ventricular aneurysms, pericardial effusion and sudden death. (8) The diagnosis of cardiac sarcoidosis (CS) is made in the co-existence of non-caseating granulomas on myocardial biopsy and/or biopsies of any extra-cardiac tissue (with the exclusion of other causes for granulomatous inflammation such as mycobacterial or fungal infection) and cardiovascular abnormalities for which other possible causes have been excluded. The guideline from the Japanese Ministry of Health and Welfare provide an excellent frame work. (9) (Table 1) The guideline combines the results of various diagnostic tests such as tissue biopsy, electrocardiography, echocardiography, and myocardial scintigraphy to either exclude or diagnose CS. Most diagnostic tests, including endomyocardial biopsy, suffer of low sensitivity, low specificity or both. The most accurate diagnostic strategy for this condition is currently unknown. We aimed to assess the accuracy of gadolinium-enhanced cardiovascular magnetic resonance (CMR) in the diagnosis of cardiac sarcoidosis (CS) with a modifiedguideline of the Japanese Ministry of Health and Welfare (1993) as gold standard. Because of the limited diagnostic yield and invasive nature of the procedure with an associated risk of morbidity, we felt not justified to systematically subject patients who were screened for CS to endo-myocardial biopsy. (10) For the purpose of the present study the Japanese guideline was modified by excluding endomyocardial biopsy as a diagnostic parameter (Table 1).

\subsection{Patients and methods}

\section{Study population}

Between July 1998 and May 2004, 58 patients with histologically proven pulmonary sarcoidosis underwent cardiac assessment in the cardiology departments of Tygerberg Hospital (1), Medical Centre Haaglanden (1), the Erasmus University Medical Centre (3) and the University Hospital Maastricht (53). The diagnosis of sarcoidosis was confirmed if the clinical presentation and chest radiographic finding were supported by histological evidence of non-caseating granulomas by transbronchial biopsy, and the possibility of infection, environmental factors or hypersensitivity reaction to medication causing granulomatous inflammation, had been eliminated. The patients had either cardiac symptoms (16), or were screened for cardiac involvement in the absence of cardiac symptoms (42). All patients underwent clinical assessment, 12-lead electrocardiography, ambulatory electrocardiographic monitoring, transthoracic echocardiography, ${ }^{201}$ thallium SPECT, and CMR. Patients underwent diagnostic coronary angiography to exclude coronary artery disease in patients 
with a (partially) reversible perfusion defect on SPECT and decreased left ventricular ejection fraction and regional wall motion abnormalities. Cardiac histology was obtained in five patients by cardiac biopsy, and three patients who had a post-mortem examination. The diagnosis of CS, according to the modified guideline of the Japanese Ministry of Health and Welfare (Table 1), was made in 12 of 58 patients included in the study.

\section{Electrocardiography and ambulatory electrocardiography}

A 12-lead surface ECG was made (MAC, Marquette, Milwaukee, Wisconsin; paper speed $25 \mathrm{~mm} / \mathrm{sec}$ ) and the findings were interpreted by two experienced cardiologists and classified as abnormal i.e. in keeping with the ECG criteria of the Japanese guideline (complete RBBB or LBBB, bifascicular block, third degree atrio-ventricular block, ventricular tachycardia, or pathological Q or ST-T abnormalities) or normal. Ambulatory ECGs were performed for 24-72 hours, and were considered abnormal when intermittent atrio-ventricular conduction delay or block, intermittent bundle branch blocks or ventricular arrhythmias such as frequent monomorphic and/or polymorphic PVCs $>100 / 24$ hours, non-sustained VTs, and/or sustained VTs were found.

\section{Echocardiography}

Studies were performed with a Sonos 5500 phased array imaging system (Hewlett Packard) and considered abnormal when regional or global systolic dysfunction, wall thickening or thinning was found. Regional differences in left ventricular wall enhancement were measured and localized according to the 17 segment model (10)

\section{Thallium myocardial scintigraphy}

After treadmill peak exercise, or during intravenous infusion of dipyridamol, ${ }^{201}$ thallium was administered and SPECT performed on a Siemens triple-detector gamma camera (MultiSPECT-3), equipped with low-energy, high resolution collimators. The images were made in a $64 \times 64$ matrix ( 60 frames $/ 45$ seconds). The thallium scan was considered suggestive of CS when areas with reversed uptake and/or irreversible perfusion defects were present, and/or reversible perfusion defects were found in patients with normal coronary arteries at angiography. Regional defects were localized according to the 17 segment model (11)

\section{Cardiac Magnetic Resonance}

Studies were performed using a 1.5 Tesla MRI scanner (Philips, Best, The Netherlands and General Electric, Milwaukee, Wisconsin, USA) with a cardiac-dedicated phased-array coil. The CMR studies were ECG triggered by standard software, and obtained in diastole to minimize artifact due to cardiac motion. Studies consisted of steady-state-free precession (SSFP) in 58 patients, spin echo (SE) in 57 patients, and fat saturated T2 weighted breath 
hold sequences in 58 patients. Short axis (SA), vertical long axis (VLA) and four chamber $(4 \mathrm{CH})$ views were obtained. SSFP sequences were performed to assess regional wall motion abnormalities. T2-weighted studies were performed to assess the presence of myocardial inflammation. Ten minutes after the additional administration of $0.1 \mathrm{mmol} / \mathrm{kg}$ gadolinium-DTPA (Schering, Berlin), SA and $4 \mathrm{CH}$ images were obtained with SE in 57 patients (slice thickness $8 \mathrm{~mm}$, gap $.8 \mathrm{~mm}$, matrix $512 \times 512$, FOV $380 \mathrm{~mm}$, voxel size $0.7 \mathrm{~mm} \times$ $0.7 \mathrm{~mm} \times 8 \mathrm{~mm}$ ) and 3-D breath hold inversion recovery-gradient echo (IR-GRE) sequences (SA, VLA, $4 \mathrm{CH}$ ) in 16 patients (slice thickness $10 \mathrm{~mm}$, no gap, matrix $256 \times 256$, FOV $400 \mathrm{~mm}$, voxel size $1.6 \mathrm{~mm} \times 1.6 \mathrm{~mm} \times 10 \mathrm{~mm}$ ) to assess for the presence of gadolinium enhancing enhancing lesions (LGE). Fifteen patients had both SE and IR-GRE studies. The inversion time (250-400 msec) was determined on an individual base to obtain optimal nulling of the unenhanced myocardial signal. Regional differences in left ventricular wall enhancement were measured and localized according to the 17 segment model (10) (MASS suite post processing software, MEDIS, Leiden, The Netherlands). The total time required for the investigation was 30-45 minutes. The studies were evaluated independently by 4 blinded observers, 3 cardiologists and 1 radiologist, with experience in ceCMR. The studies were considered to be abnormal when at least two independent observers described identical abnormalities.

\section{Statistical analysis}

All statistical analyses were performed using the statistical software package SSPS version 11.5. Group data are expressed as mean \pm SD. Continuous variables were assessed using the parametric $t$ test for independent samples or Mann Whitney test were appropriate, and all categorical variables were assessed using the chi-square test. Statistical significance was defined as a $\mathrm{p}$ value less than 0.05 .

\subsection{Results}

\section{Patient characteristics}

A total of 58 patients were assessed. Twelve of the patients were diagnosed with CS according to the modified guidelines. The demographic, clinical and diagnostic findings in the group with CS and without CS are shown in Tables 2 and 3. The ages, gender, race, the time between cardiac evaluation and the diagnosis of sarcoidosis, and pulmonary stage did not differ significantly between the groups. Functional class was significantly poorer in the patient group with CS compared to the group without CS due to heart failure.

\section{Cardiac Magnetic Resonance}

Table 4 summarizes the findings of the CMR studies. In $40(69 \%)$ studies 4 out of 4 observers, in $13(22 \%)$ studies 3 out of 4 observers, and in $5(9 \%)$ studies 2 out of 4 observers 
agreed on the absence or the presence and localization of LGE. Late gadolinium enhancing lesions were present in 19 patients (mean 3.6 segments/pt, range 1-12). Nine of 19 patients had evaluation with SE, 9 with SE and IR-GRE, and 1 patient had only IR-GRE. In 5 of the SE patients 2, and in 3 of the SE patients 3 observers agreed on the presence and localization of LGE, while in 9 of the 10 patients who had both SE and IR-GRE or only IRGRE all observers agreed on the presence and localization of LGE. Most enhancing lesions were located in the basal and lateral left ventricular segments (segments 1-7,11,12,16; 60/71 enhancing segments (73\%)). (Figure 1) In 10 patients a significantly reduced left ventricular systolic function (LVEF 20-51\%, mean 36\%) was reported, 7 (54\%) with CS, one patient with dilated cardiomyopathy, one with valvular hart disease, and 1 with coronary artery disease. In 3 patients regional loss of wall thickness was found, 2 patients with CS and one with coronary artery disease. Twelve of the 22 patients with abnormal CMR studies were diagnosed with CS according to the modified guideline. Overall the diagnosis of cardiac sarcoidosis according to the modified guideline was made in 12 of 58 sarcoidosis patients (21\%). The sensitivity and specificity of ceCMR was $100 \%$ (one sided $95 \%$ CI, 78 $100 \%)$ and $78 \%(95 \%$ CI, 64-89\%) the PPV and NPV 55\% respectively 100\%, with an overall accuracy of $83 \%$. CMR showed one to six contrast-enhancing myocardial lesions in 6 patients, who had not been diagnosed with CS according to the modified guideline. In 8 patients CMR (5 with SE, 3 with SE and IR-GRE) diagnosed LGE, while ${ }^{123}$ thallium scintigraphy was normal. In 6 of the 8 patients LGE involved only 1 or 2 segments, while in 2 patients respectively 6 and 8 segments were involved. In 3 patients LGE involved 1 myocardial layer, sub-endocardial or mid-myocardial, in 1 patient both sub-endocardial and mid-myocardial layers were involved, and in 4 patients patchy involvement of all 3 myocardial layers was present, without confluent transmural involvement.

\subsection{Discussion}

The prognosis of sarcoidosis is mainly determined by pulmonary and cardiac involvement. $(\mathbf{1 2 , 1 3 )}$ Multivariate analysis identified New York Heart Association functional class, LV enddiastolic diameter and the presence of sustained ventricular tachycardias as independent predictors of mortality. (14) It is important to accurately diagnose cardiac involvement. The guideline published by the Japanese Ministry of Health and Welfare (1993) provides us with an excellent frame work for the diagnosis, but the techniques utilized lack sensitivity and specificity in the absence of a diagnostic endo-myocardial biopsy. Magnetic resonance imaging is a robust imaging technique that enables us to evaluate cardiac structure, function, and tissue characteristics with high image resolution. It may guide the cardiologist when taking endo-myocardial biopsies and increase the sensitivity of this technique. $(\mathbf{1 5 , 1 6 )}$ Serial CMR studies are possible because of the absence of exposure to ionizing radiation, and may evaluate disease activity and extent in response to therapy. Postmortem studies in sarcoid patients have revealed the presence of varying amounts of myocardial scar tissue, which is considered to be the substrate for lethal ventricular arrhythmias. $(4,5,12,13,14)$ Gadolinium-DTPA (Gd-DTPA) is an extra-cellular CMR contrast agent. Histological assessment of Gd-DTPA enhanced myocardium has been correlated with local fibrosis and 
active myocarditis. $(\mathbf{1 6 , 1 7 , 1 8 )}$ Wagner et al. have demonstrated the superior ability of Gdenhanced CMR to diagnose even small amounts of myocardial scar tissue in patients with coronary artery disease when compared to myocardial scintigraphy. (19) Several casereports and small single-centre patient series have demonstrated the diagnostic potential of CMR in cardiac sarcoidosis. (20-28) Sofar no study has systematically evaluated the diagnostic accuracy of CMR for the presence of CS, with the diagnostic Japanese guideline as gold standard. The current study is part of a prospective initiative that aims to evaluate the prevalence of cardiac involvement in patients with sarcoidosis, and determine the most accurate and cost-effective diagnostic strategy for CS. The diagnostic accuracy of CMR compares well to the techniques employed in the diagnostic framework of the Japanese guideline. The fact that the combination of ECG and CMR saves valuable time at equal cost compared to our previously employed diagnostic pathway of ECG, echocardiography and SPECT (cost in The Netherlands respectively $€ 222$,- and $€ 205,-$ ) makes it an attractive alternative. The low PPV of 55\% reflects the superior spatial resolution of CMR. CMR is able to detect small myocardial lesions which have not resulted in conduction delay, repolarization abnormalities, or regional changes in wall thickness or function, and hence are not detected by ECG, echocardiography or SPECT. Unfortunately even small myocardial scars may result in arrhythmias, and the follow up of this patient population will determine the significance of our findings. For this reason, CMR may become very important for the early detection of cardiac involvement and for identifying pulmonary sarcoidosis patients at risk of sudden death. Most of the enhancing lesions (73\%) were localized in the basal and lateral left ventricular wall. These findings correlate with the findings at postmortem assessment of sarcoidosis patients, where most lesions were found in the mentioned segments. $(4,5)$ Although in some patients LGE was transmural, resulting in loss of wall thickness and regional wall motion abnormalities, lesions in most patients were patchy, affecting several myocardial layers. The presence of patchy LGE has also been reported in patients with dilated cardiomyopathy, but our findings clearly differ from the distribution of LGE in patients with coronary artery disease. (29) Considering the diagnostic limitations of electrocardiography, $25 \%$ of patients with extensive cardiac involvement in Roberts study had normal ECGs, and the superior spatial resolution of CMR compared to scintigraphy, new diagnostic guidelines need to be compiled that include CMR

\subsection{Conclusions}

In patients with sarcoidosis CMR is a useful, cost-effective diagnostic tool to determine cardiac involvement. The combination of ECG with CMR was as accurate but more timeand cost-effective compared to ECG, ambulatory ECG, echocardiography and SPECT for the diagnosis of CS. CMR diagnosed small myocardial lesions, not diagnosed by ECG, echocardiography or SPECT, that might be of prognostic relevance. 


\subsection{Study limitations}

Ideally CMR findings should be correlated with CMR guided EMB i.e. myocardial histology. In our study CMR findings were compared to clinical guidelines. In only four patients myocardial histology was obtained.

\subsection{Recommendations for further study}

There is a need for multi-centre studies that will determine the diagnostic accuracy of CMR and studies into the prognostic implications of ceCMR detected CS not detected by conventional methods. Early treatment may possibly prevent sudden death and deterioration of ventricular function and result in improved prognosis.

\subsection{Acknowledgements}

The authors gratefully acknowledge Dr H.Kuehl, MD, PhD, Aachen University Medical Centre, and Dr A.M.Beek, MD, Free University Medical Centre, Amsterdam, for assessing the CMR studies. We thank professor A.F.Doubell, MMed (Int), PhD, Cardiac Unit, Tygerberg Hospital, Cape Town, Republic of South Africa, and Dr JHM Schreur, Medical Centre Haaglanden, The Hague, for allowing us to include their patients in our study. 


\section{References}

1. Johns CJ, Michele TM. The clinical management of sarcoidosis: a 50-year experience at the Johns Hopkins Hospital. Medicine 1999;78:65-111.

2. Shammas RL, Movahed A. Sarcoidosis of the heart. Clin Cardiol 1993; 16:462-72.

3. Hagemann GJ, Wurm K. The clinical, electrocardiographic and pathological features of cardiac sarcoid. In: Jones Williams W, Davies BH, eds. Sarcoidosis and other granulomatous disorders. Proceedings of the 8th International Conference. Cardiff: Alfa Omega Publishing, 1980:601-6.

4. Silverman KJ, Hutchins GM, Bulkley BH. Cardiac sarcoid: a clinico-pathologic study of 84 unselected patients with systemic sarcoidosis. Circulation 1979;58:1204-11.

5. Roberts WC, McAllister HA Jr, Ferrans VJ. Sarcoidosis of the heart: a clinico-pathological study of 35 necropsy patients (group 1) and review of 78 previously described necropsy patients (group II). Am J Med 1977;63:86-108.

6. Matsui Y, Iwai K, Tachibana T, et al. Clinico-pathological study on fatal myocardial sarcoidosis. Ann NY Acad Sci 1976;278:455-69.

7. Tachibana T, Iwai K, Takemura T. Study on the cause of death in patients with sarcoidosis in Japan. XII World Congress on Sarcoidosis, Kyoto, Japan, September 8-13,1991. Abstract.

8. Sharma OP, Maheshwari, Thaker K. Myocardial sarcoidosis. Chest 1993; 103(1):253-7.

9. Hiraga H, Yuwai K, Hiroe M, et al. Guideline for the diagnosis of cardiac sarcoidosis: study report on diffuse pulmonary diseases [in Japanese]. Tokyo, Japan: The Japanse Ministry of Health and Welfare; 1993;23-4.

10. Deng JC, Baughman RP, Lynch JP. Cardiac involvement in sarcoidosis. Sem Resp Crit Care Medicine 2002;23:513-26.

11. Cerqueira MD, Weissman NJ, Dilsizian V, et al. AHA writing group on myocardial segmentation and registration for cardiac imaging. Standardized myocardial segmentation and nomenclature for tomographic imaging of the heart. Circulation 2002;105:539-42.

12. Sekiguchi M, Yazaki Y, Isobe M, Hiroe M. Cardiac sarcoidosis: diagnostic, prognostic and therapeutic considerations. Cardiovasc Drugs Ther 1996;10:495-510.

13. Perry A, Vuitch F. Causes of death in patients with sarcoidosis: a morphologic study of 38 autopsies with clinicopathologic correlations. Arch Pathol Lab Med 1995;119:167-72.

14. Yazaki Y, Isobe M, Hiroe M, et al. Prognostic determinants of long-term survival in Japanese patients with cardiac sarcoidosis treated with prednisone. Am J Cardiol 2001;88:1006-10.

15. Riedy K, Fisher MR, Belic N, Koenigsberg DI. MR Imaging of myocardial sarcoidosis. Am J Roentgenol 1988;151:915-6.

16. Mahrholdt H, Goedeke C, Wagner A, et al. CMR in human myocarditis: a comparison to histology and molecular pathology. Circulation 2004;16:1250-8.

17. Aso H, Takeda K, Ito T, Shiraishi T, Matsumura K, Nakagawa T. Assessment of myocardial fibrosis in cardiomyopathic hamsters with gadolinium-DTPA enhanced magnetic resonance imaging. Invest Radiol 1998;33:22-32.

18. Moon JCC, Reed E, Sheppard MN, Elkington AG, Yen Ho S, Burke M, et al. The histologic basis of late gadolinium enhancement cardiovascular magnetic resonance in hypertrophic cardiomyopathy. J Am Coll Cardiol 2004;43:2260-4.

19. Wagner A, Mahrholdt H, Holly TA, et al. Contrast-enhanced MRI and single photon emission computed tomography (SPECT) perfusion imaging for the detection of subendocardial myocardial infarcts: an imaging study. Lancet 2003;361:374-9.

20. Chandra M, Silverman ME, Oshinski J, et al. Diagnosis of cardiac sarcoidosis aided by MRI. Chest 1996;110:562-5.

21. Doherty MJ, Kumar SK, Nicholson AA, et al. Cardiac sarcoidosis: the value of magnetic resonance imaging in diagnosis and assessment of response to treatment. Resp Med 1998;92:697-99.

22. Inoue SI, Shimada T, Murakami Y. Clinical significance of gadolinium-DTPA enhanced MRI for detection of myocardial lesion in patients with sarcoidosis. Clin Radiol 1999;54:70-72.23. Matsuki M, Matsuo M. MR findings of myocardial sarcoidosis. Clin Radiol 2000;55:323-5.

24. Shimada T, Shimada K, Sakane T, et al. Diagnosis of cardiac sarcoidosis and evaluation of the effects of steroid therapy by gadolinium-DTPA-enhanced magnetic resonance imaging. Am J Med 2001;110:520-7. 


\section{Chapter 5}

25. Vignaux $\mathrm{O}$, Dhôte R, Duboc D, et al. Detection of myocardial involvement in patients with sarcoidosis applying T2-weighted, contrast-enhanced and cine magnetic resonance imaging: initial results of a prospective study. J Comput Assist Tomograph 2002;26:762-7.

26. Vignaux $\mathrm{O}$, Dhôte $\mathrm{R}$, Duboc D, et al. Clinical significance of myocardial magnetic resonance abnormalities in patients with sarcoidosis. A 1-year follow-up study. Chest 2002;122:1895-1901.

27. Sköld CM, Larsen FF, Rasmussen E, Pehrson SK, Eklund AG Determination of cardiac involvement in sarcoidosis by MRI and Doppler echocardiography. J Int Med 2002;252:465-71.

28. Dhôte R, Vignaux $\mathrm{O}$, Blanche $\mathrm{P}$, et al. Value of MRI for the diagnosis of cardiac involvement in sarcoidosis. Revue Med Int 2003;24:151-7.

29. McCrohon JA, Mon JCC, Prasad SK, et al. Differentiation oh heart failure related to dilated cardiomyopathy and coronary artery disease using gadolinium-enhanced magnetic resonance. Circulation 2003;108:54-9. 
Table 1: Modified guidelines for the diagnosis of cardiac sarcoidosis based on the study report on diffuse pulmonary diseases from the Japanese Ministry of Health and Welfare,1993. (9)

1. Histologic diagnosis group: endomyocardial biopsy demonstrates epithelioid granulomata without caseating granulomata.

2. Clinical diagnosis group: in patients with histologic diagnosis of extra-cardiac sarcoidosis, cardiac sarcoidosis is suspected when ' $a$ ' and at least one of criteria 'b' to ' $d$ ' are present, and other etiologies such as hypertension and coronary artery disease have been excluded:

a. Complete RBBB, $L B B B$ (included by the authors), left-axis deviation, AV-block, VT, PVC, or pathological Q or ST-T change on resting- or ambulatory ECG.

b. Abnormal wall motion, regional wall thinning, or dilation of the left ventricle.

c. Perfusion defect by ${ }^{201}$ thallium-myocardial scintigraphy or abnormal accumulation by $67 \mathrm{Ga}$-citrate or 99m'Tc-PYP myocardial scintigraphy.

d. Abnormal intracardiac pressure, low cardiac output, or abnormal wall motion or depressed ejection fraction of the left ventricle.

(the original guidelines mentioned: e.Interstitial fibrosis or cellular infiltration over moderate grade, even if the findings are nonspecific)

Table 2: Summary of demographic and background medical data.

\begin{tabular}{|c|c|c|c|}
\hline & $\begin{array}{l}\text { Group with cardiac } \\
\text { sarcoidosis }(\mathrm{n}=12)\end{array}$ & $\begin{array}{l}\text { Group without } \\
\text { cardiac sarcoidosis } \\
(\mathrm{n}=46)\end{array}$ & $\mathrm{P}$ value \\
\hline Mean age (years) & $49 \pm 6$ & $47 \pm 10$ & ns \\
\hline $\begin{array}{l}\text { Functional class } \\
\text { No complaints } \\
\text { Modified NYHA (class } 0-\text { IV) } \\
\text { NYHA I } \\
\text { NYHA II } \\
\text { NYHA III } \\
\text { NYHA IV }\end{array}$ & $\begin{array}{l}9(69 \%) \\
0.9 \pm 1.6 \\
0 \\
2 \\
0 \\
2\end{array}$ & $\begin{array}{l}40(87 \%) \\
0.2 \pm 0.5 \\
1 \\
3 \\
0 \\
0\end{array}$ & $\mathrm{P}<0.01$ \\
\hline Time (years) since diagnosis of sarcoidosis & $9 \pm 8$ & $6 \pm 9$ & ns \\
\hline Pulmonary stage (I-IV) & $1.8 \pm 1$ & $2.2 \pm 1.2$ & ns \\
\hline Steroid treatment & $12(92 \%)$ & $31(67 \%)$ & ns \\
\hline Hypertension & 0 & $10(23 \%)$ & $\mathrm{P}<0.05$ \\
\hline Palpitations & $4(31 \%)$ & $13(30 \%)$ & ns \\
\hline Syncope & $1(8 \%)$ & $2(5 \%)$ & ns \\
\hline ACE maximal (IU/l) & $35 \pm 40$ & $30 \pm 17$ & ns \\
\hline Abnormal ECG & $13(100 \%)$ & $3(7 \%)$ & $\mathrm{P}<0.0001$ \\
\hline Abnormal ambulatory ECG & $7(58 \%)$ & $6(13 \%)$ & $\mathrm{P}<0.01$ \\
\hline Abnormal echocardiogram & $6(46 \%)$ & $3(7 \%)$ & $\mathrm{P}<0.01$ \\
\hline Abnormal thallium scintigram & $3(43 \%)$ & $5(11 \%)$ & ns \\
\hline \multicolumn{4}{|l|}{ CMR } \\
\hline Regional contrast enhancement & $12(92 \%)$ & $8(17 \%)$ & $\mathrm{P}<0.0001$ \\
\hline Number of enhancing segments & $3.3 \pm 3.6$ & $0.5 \pm 1.3$ & $\mathrm{P}<0.0001$ \\
\hline Decreased LVEF & $7(54 \%)$ & $3(7 \%)$ & $\mathrm{P}<0.0001$ \\
\hline Mean LVEF (\%) & $48 \pm 15$ & $59 \pm 5$ & $\mathrm{P}<0.0001$ \\
\hline Loss of wall thickness & $2(15 \%)$ & $1(2 \%)$ & ns \\
\hline
\end{tabular}




\section{Chapter 5}

Table 3: 12 patients with cardiac sarcoidosis (modified Japanese guideline).

\begin{tabular}{|c|c|c|c|c|c|c|c|c|c|c|}
\hline pt & $\begin{array}{l}\text { age } \\
\text { (yrs) }\end{array}$ & sex & $\begin{array}{l}\text { time since } \\
\text { diagnosis of } \\
\text { sarcoidosis } \\
(\mathrm{yrs})\end{array}$ & symptoms & thallium & echo & CMR & ECG & Holter & EMB \\
\hline 1 & 53 & M & 10 & heart failure & & 1 & 1,2 & $\Delta \mathrm{ST}-\mathrm{T}$ & 0 & $\begin{array}{l}\text { non-caseating } \\
\text { granulomas }\end{array}$ \\
\hline 2 & 50 & $\mathrm{~F}$ & 4 & palpitations & & 1 & 1 & $\Delta \mathrm{ST}-\mathrm{T}$ & VT & \\
\hline 3 & 52 & $\mathrm{~F}$ & 30 & heart failure & normal & 1 & 1,2 & pseudo-infarct & $\mathrm{nsVT}$ & fibrosis \\
\hline 4 & 55 & $\mathrm{~F}$ & 7 & heart failure & abnormal & 1 & 1,2 & LBBB & 0 & \\
\hline 5 & 41 & M & 1 & palpitations & abnormal & 1 & 1,3 & $\Delta \mathrm{ST}-\mathrm{T}$ & nsVT & fibrosis \\
\hline 6 & 42 & M & 5 & palpitations & normal & 0 & 1 & bifasc block & 0 & \\
\hline 7 & 43 & M & 14 & heart failure & & 1 & 2 & LBBB & nsVT & fibrosis \\
\hline 8 & 43 & M & 6 & palpitations & normal & 0 & 1 & $\mathrm{RBBB}$ & 0 & \\
\hline 9 & 54 & M & 15 & heart failure & normal & 1 & 1,2 & $3^{\text {rd }}$ AV block & 0 & \\
\hline 10 & 54 & M & 5 & heart failure & normal & 0 & 1,2 & $3^{\text {rd }}$ AV block & 0 & \\
\hline 11 & 41 & M & 10 & none & abnormal & 1 & 1 & $\Delta \mathrm{ST}-\mathrm{T}$ & 0 & \\
\hline 12 & 52 & M & 4 & syncope & & 1 & $1,2,3$ & pseudo-infarct & $\mathrm{nsVT}$ & \\
\hline
\end{tabular}




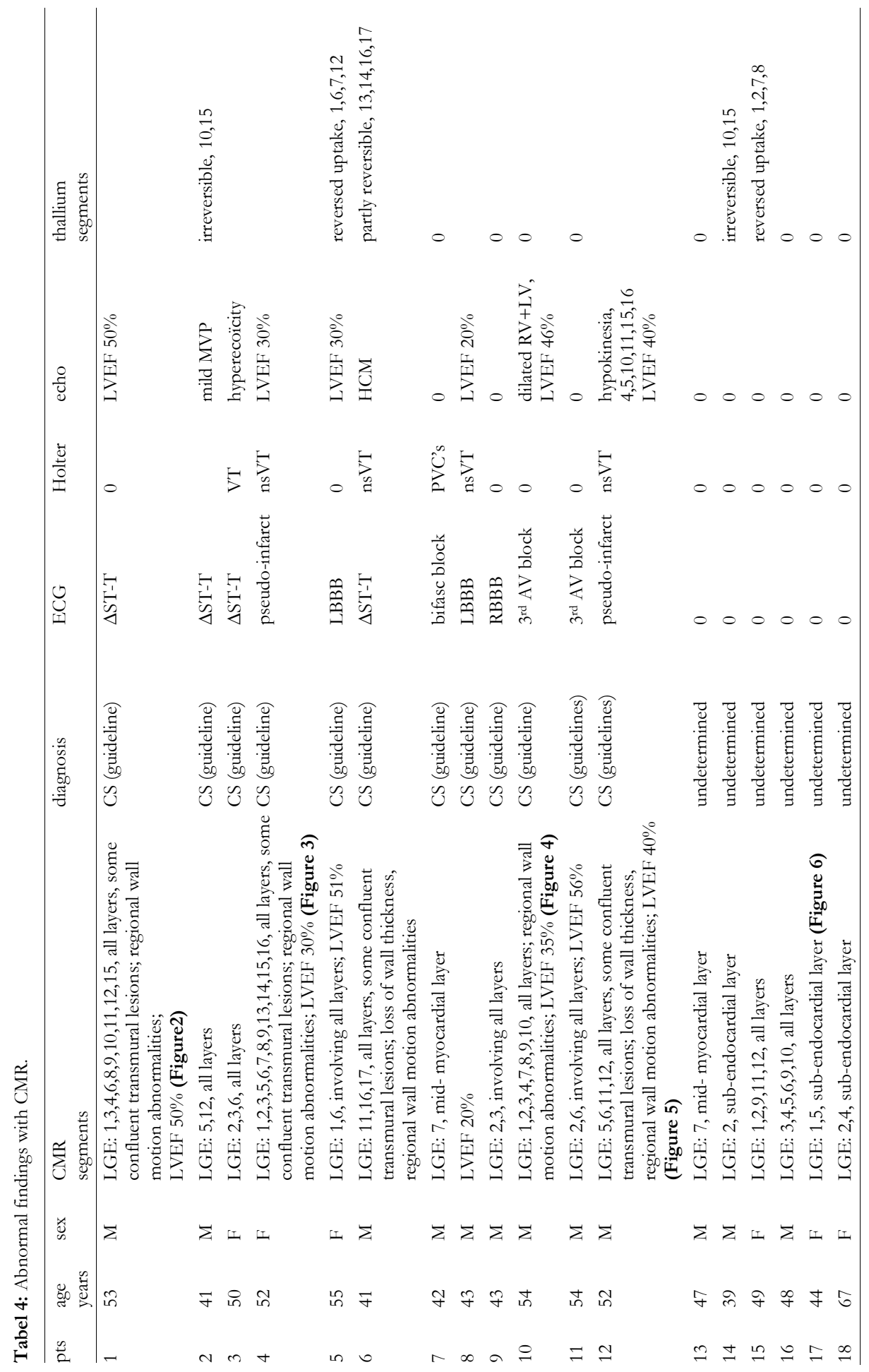


Chapter 5

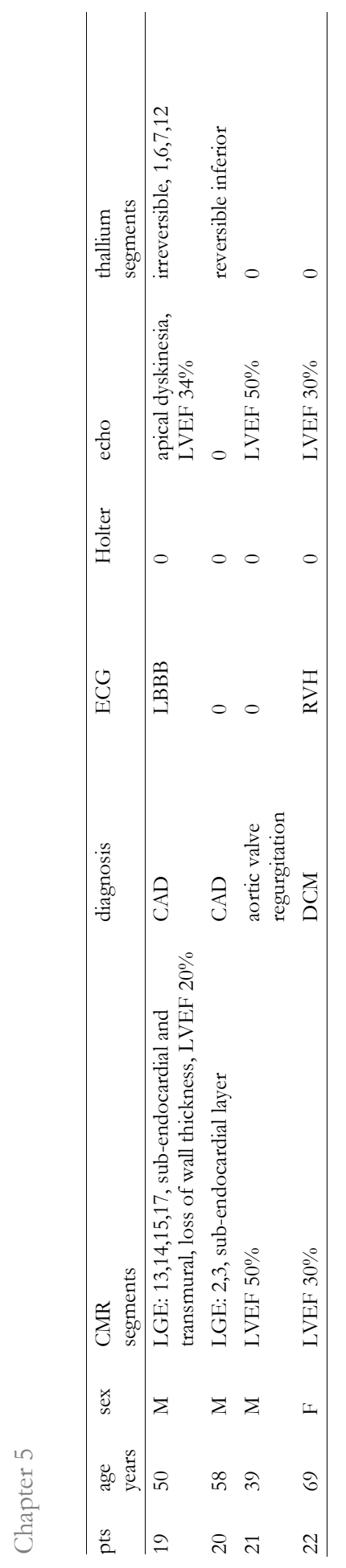



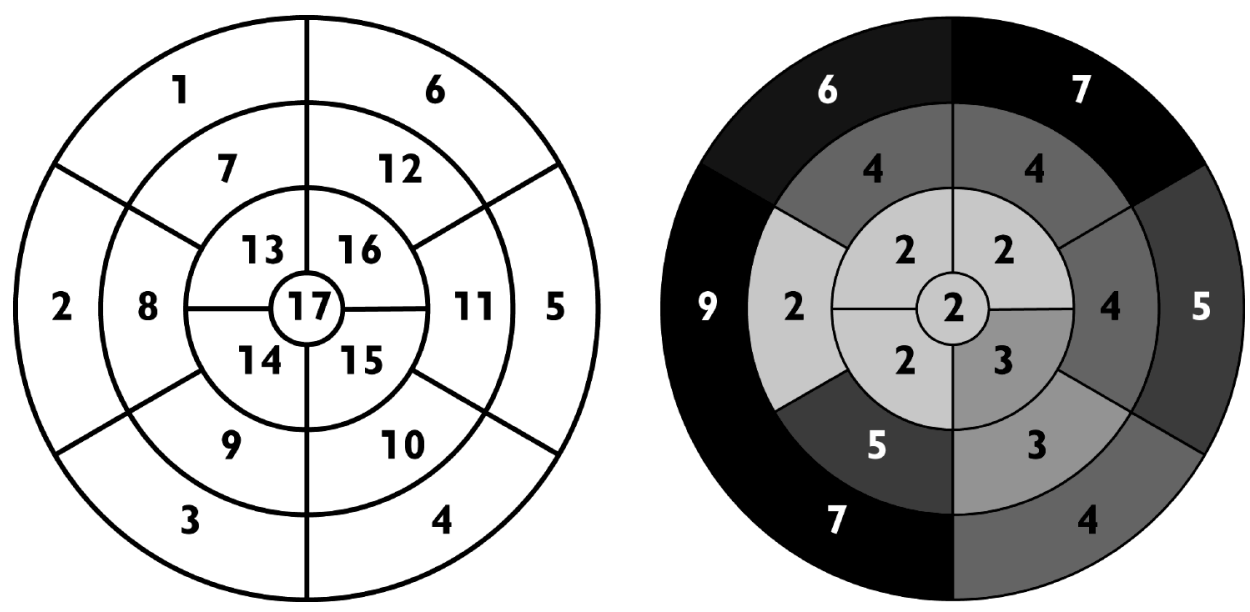

Figure 1. Bulls eye plots demonstrating left ventricular segmentation (left) (11) and the distribution of late enhancement per segment in 19 sarcoidosis patients (right).

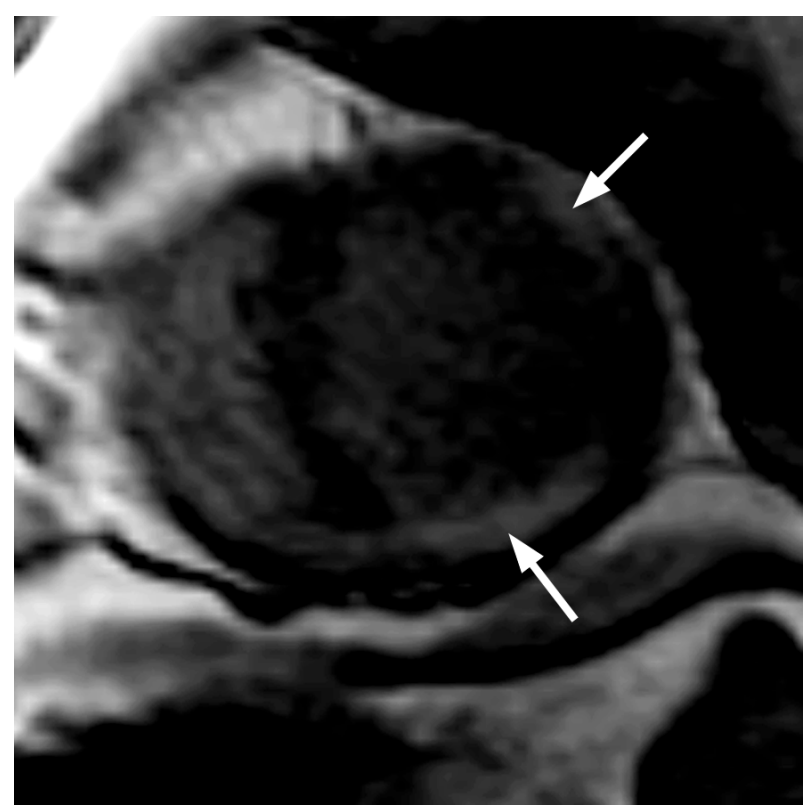

Figure 2A: CMR study (IR-GRE, SA view, 10 minutes after administration of $0.1 \mathrm{mmol} / \mathrm{Gd}-\mathrm{DTPA}$ ) of patient 1 , a 53-year old man with a history of stage 2 pulmonary sarcoidosis, who presented with congestive cardiac failure. Arrows point towards the enhanced myocardial segments (10, 11 and 12). 


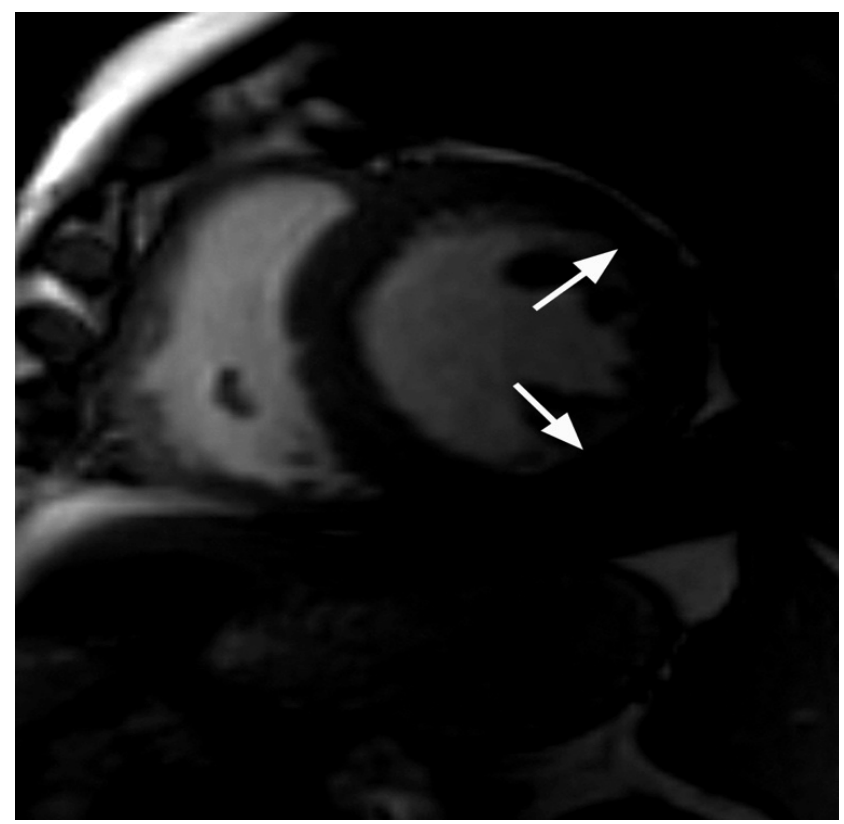

Figure 2B: The end-systolic frame (SSFP, SA view) of this slice demonstrated regional loss of wall thickness and hypokinesia of the Gd- enhanced segments (10,11 and 12).

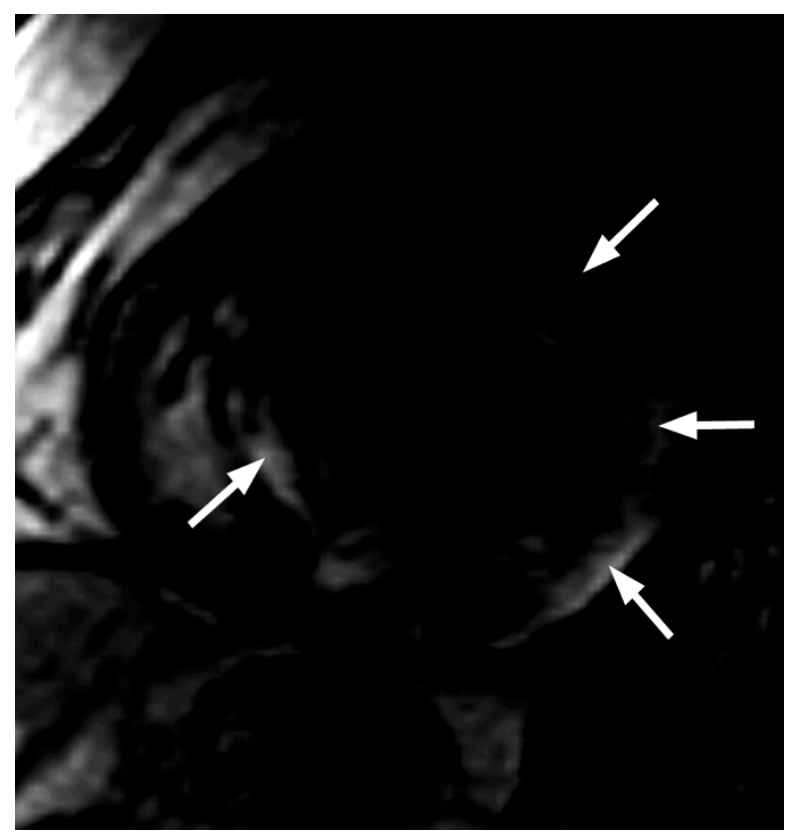

Figure 3: CMR study (IR-GRE, SA view,10 minutes after administration of $0.1 \mathrm{mmol} / \mathrm{Gd}-\mathrm{DTPA}$ ) of patient 4 , demonstrated extensive late gadolinium enhancement, involving $33 \%$ of the left ventricle. 


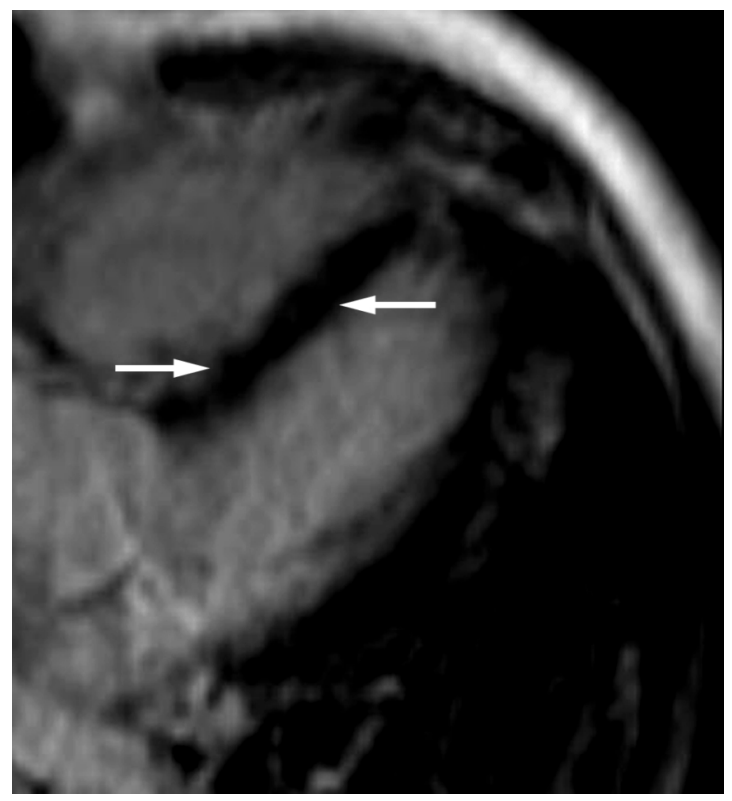

Figure 4: CMR study (IR-GRE, 4CH view, 10 minutes after administration of $0.1 \mathrm{mmol} / \mathrm{Gd}-\mathrm{DTPA}$ ) of patient 10, a 54-year old man with a history of stage 3 pulmonary sarcoidosis, who was referred with grade III atrioventricular block. Arrows point towards the Gd-DTPA enhanced myocardial segments $(2,8)$.

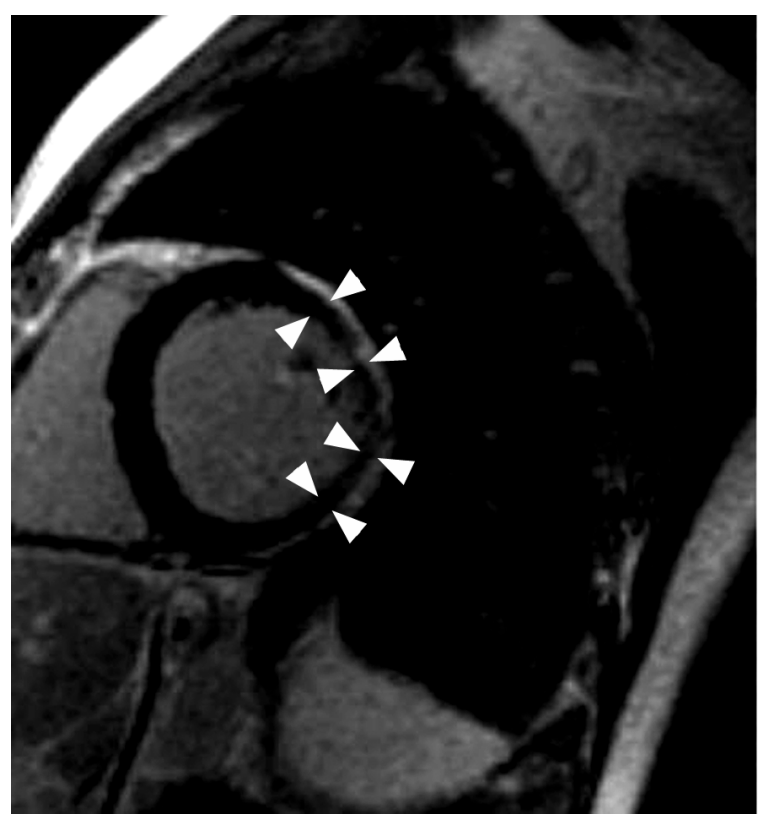

Figure 5A: CMR study (3 D breath-hold IR-FGRE, SA view, 10 minutes after administration of $0.1 \mathrm{mmol} / \mathrm{Gd}-$ DTPA) of patient 12 demonstrated loss of wall thickness and late gadolinium enhancement (segments 11 and 12). This patient survived out of hospital cardiac arrest secondary to ventricular fibrillation. 


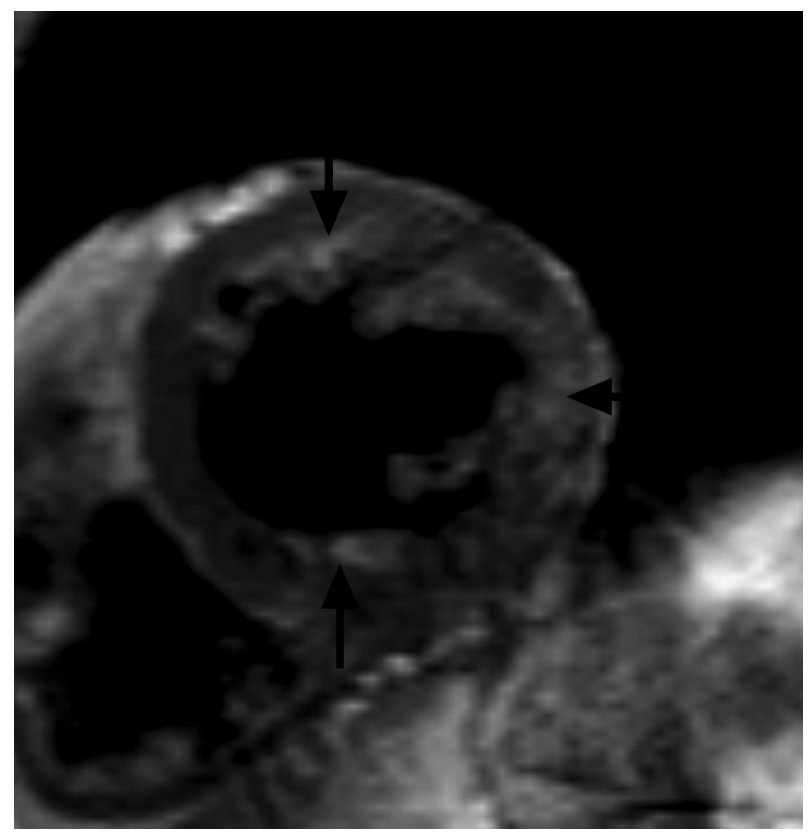

Figure 5B: CMR study (SSFP, 4 chamber view) of patient 12 demonstrated loss of wall thickness and late gadolinium enhancement (segments 11 and 12). This patient survived out of hospital cardiac arrest secondary to ventricular fibrillation.

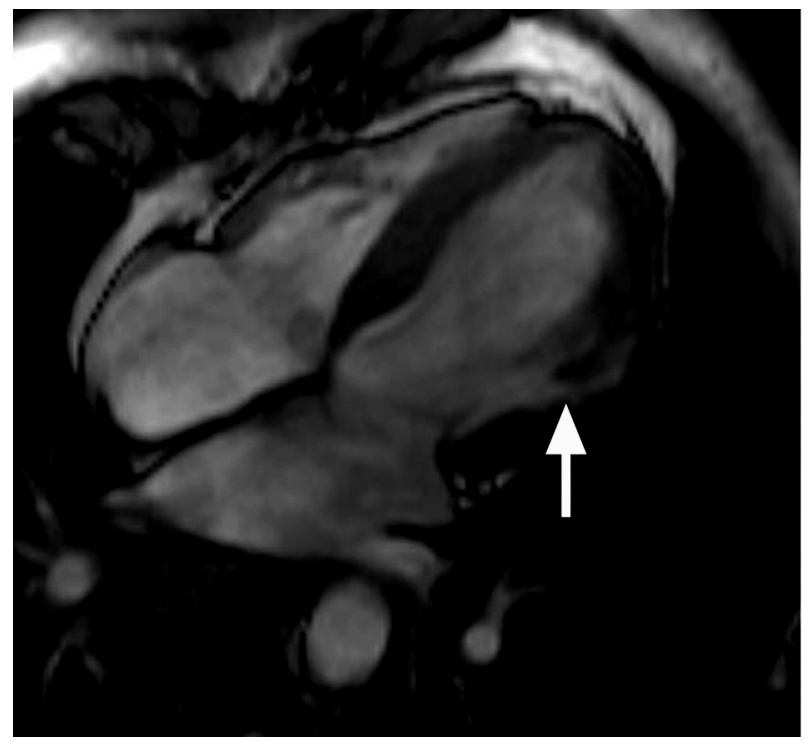

Figure 6: CMR study (SE, SA view) of patient 17 demonstrated late gadolinium enhancement (segments 1,4) in a patient in whom standard assessment had not revealed abnormalities. 


\section{Chapter 6}

\section{Contrast-Enhanced Cardiac Magnetic Resonance: Distinction between cardiac sarcoidosis and infarction scar}

Sarc Vasc Diff Lung Dis 2017;34:307-314.

Jan-Peter Smedema, MD, Robert-Jan van Geuns, MD, PhD, Rene Truter, MMed (Rad), Bongani M. Mayosi, MMed (Int), D Phil (Oxon), Harry J.G.M Crijns, MD, PhD.

Departments of Cardiology (JPS, HJGM), Maastricht University Medical Centre, The Netherlands; Department of Cardiology and Radiology (RJvG), Erasmus Medical Centre, Rotterdam, The Netherlands; The Cardiac Clinic (BMM), Department of Medicine, Groote Schuur Hospital and University of Cape Town, Cape Town, South Africa; Schnetler, Corbett and Associates (RT), Panorama Medi-Clinic, Cape Town, South Africa. 


\begin{abstract}
Objectives: To review the value of delayed contrast-enhanced cardiac magnetic resonance (CECMR) in differentiating patients with cardiac sarcoidosis (CS) from those with coronary artery disease and recent myocardial infarctions.
\end{abstract}

Background: Late gadolinium enhancement (LGE) accurately delineates myocardial necrosis or fibrosis. The pattern of LGE in ischemic and non-ischemic myocardial disease is different, and might be helpful in distinguishing CS from ischemic disease.

Methods: The CECMR studies of 30 patients with CS were compared to those performed in 30 consecutive infarct patients, who had been managed with primary coronary interventions, and 10 healthy controls. Two experienced blinded observers classified patients by assessing the distribution of LGE.

Results: LV LGE was present in 29/30 CS (mean 3.8 segments, range $0-12$ ), all infarct (mean 4.3 segments, range $0-9$ ), and none of the patients in the control group. The amount of LV LGE did not differ significantly between CS and infarct patients $(19 \pm 11 \%$ and $19 \pm 12 \%, \mathrm{P}=0.8)$. The CS group exhibited a predominantly patchy, 3 layer LGE $(\mathrm{P}=$ 0.01), whereas confluent transmural LGE $(\mathrm{P}=0.04)$ with a vascular distribution $(\mathrm{P}<$ 0.001) was prevalent in the infarct group. Significantly more RV LGE (P = 0.01) and dilation $(\mathrm{P}=0.02)$ were found in the CS group. The two observers classified patients correctly as CS in $72 \%$ and $83 \%$ of cases, as ischemic in nature in $77 \%$ and $80 \%$ of cases, and as normal in $90 \%$ and $100 \%$ respectively.

Conclusions: Gadolinium CMR was helpful in differentiating patients with CS from patients with ischemic heart disease and previous myocardial infarctions. In a subgroup of ischemic patients the pattern of LGE was atypical, and suggestive of non-ischemic etiology.

\title{
Contents
}

$\begin{array}{ll}\text { Abstract } & 148\end{array}$

6.1 Introduction 149

6.2 Patients and methods 149

6.3 Results 151

6.4 Discussion 152

6.5 Conclusion 153

References 154 


\subsection{Introduction}

Sarcoidosis is a multi-system granulomatous disorder of unknown etiology with cardiac involvement in approximately twenty to thirty percent of patients. (1) The clinical features of sarcoid heart disease include congestive heart failure, cor pulmonale, supraventricular and ventricular arrhythmias, atrioventricular and intraventricular conduction disease, ventricular aneurysms, pericardial effusion and sudden death. (1) The diagnosis of cardiac sarcoidosis (CS) is made in the co-existence of non-caseating granulomas on myocardial biopsy or biopsies of any extra-cardiac tissue (with the exclusion of other causes for granulomatous inflammation such as mycobacterial or fungal infection) and cardiovascular abnormalities for which other possible causes have been excluded. (2) The value of gadolinium-enhanced cardiac magnetic resonance (CMR) in the diagnosis and management of this condition has been demonstrated. (2-4) Late gadolinium enhancement (LGE) by CMR is the most accurate non-invasive method to evaluate myocardial necrosis or fibrosis caused by acute myocardial infarction, chronic myocardial infarction or non-ischemic myocardial disease. (5) The distribution of LGE was valuable in differentiating between ischemic and non-ischemic myocardial scarring. (6-9) Since a significant number of patients with CS present with symptoms of heart failure or chest pain, similar in nature to those in patients with coronary artery disease, we aimed to determine whether CMR, and specifically the pattern of MDE, would allow us to distinguish the CS patients from patients with coronary artery disease and recent myocardial infarcts.

\subsection{Patients and methods}

\section{Patient population}

Between July 1998 and November 2004 thirty patients were diagnosed with CS according to modified guidelines, based on the Study Report on Diffuse Pulmonary Diseases of the Japanese Ministry of Health and Welfare (1993). (2) We compared the CMR findings in the CS patients with those of 30 consecutive patients who had presented with myocardial infarcts, and who had CECMR studies during the study period. We included CECMR studies of 10 healthy control subjects. All patients in the infarct group had recently been diagnosed with myocardial infarction as defined by the European Society of Cardiology and American College of Cardiology, and underwent coronary angiography with (primary) percutaneous coronary interventions. (10) Twenty patients had myocardial infarction in the distribution of a single coronary artery (right coronary artery: 6 patients, left anterior descending artery: 7 patients, left circumflex artery: 7 patients), while ten patients had infarcts in the distribution of two coronary arteries (right coronary artery and left anterior descending artery: 4 patients, left anterior descending artery and left circumflex artery: 4 patients, right coronary artery and left circumflex artery: 2 patients). Since the study concerned retrospective analysis of clinical data in the CS and infarct groups, the Institutional Review Board waived the need for consent according to Dutch legislation. The Board approved the CMR studies in the control subjects, and all controls provided written informed consent. 


\section{CMR protocol}

Studies were performed using a 1.5 Tesla MRI scanner [(Philips, Best, The Netherlands (53 patients), Siemens, Erlangen, Germany (3 patients, 10 controls) and General Electric, Milwaukee, Wisconsin, USA (4 patients)] with a cardiac-dedicated phased-array coil. The CMR studies were ECG triggered by standard software. All patients underwent steadystate-free precession studies of short axis, vertical long axis and four chamber views, to assess regional wall motion abnormalities. Before and ten minutes after the administration of $0.1 \mathrm{mmol} / \mathrm{kg}$ gadolinium-DTPA (Schering, Berlin), short axis, vertical long axis and 4 chamber images were obtained with Spin Echo in 5 patients (slice thickness $8 \mathrm{~mm}$, gap 8 $\mathrm{mm}$, matrix $512 \times 512$, FOV $380 \mathrm{~mm}$, voxel size $0.7 \mathrm{~mm} \times 0.7 \mathrm{~mm} \times 8 \mathrm{~mm}$ ), and 3-D breath hold inversion recovery-gradient echo (IR-GRE) sequences, obtained in diastole to minimize artifact due to cardiac motion, in the remaining 55 patients and 10 controls (slice thickness $10 \mathrm{~mm}$, no gap, matrix $256 \times 256$, FOV $400 \mathrm{~mm}$, voxel size $1.6 \mathrm{~mm} \times 1.6 \mathrm{~mm} \times$ $10 \mathrm{~mm})$ to assess for the presence of LGE. The inversion time (250-400 msec) was determined on an individual basis to obtain optimal nulling of the unenhanced myocardial signal. The total time required for the investigation was 30-45 minutes.

\section{CMR Analysis}

CMR studies were analyzed off-line using commercially available software (CAAS MRV 3, Pie Medical Imaging, Maastricht, The Netherlands). Ventricular parameters were assessed in a standard way. (11) Regional wall motion abnormalities, loss of wall thickness and left ventricular (LV) LGE were localized according to the standard 17 segment model. (12) After delineating the endocardial and epicardial LV contours manually, the hyper-enhanced myocardium was depicted by changing the threshold setting for signal intensity. Signal intensities of LV and right ventricular (RV) LGE, and remote unenhanced LV and RV myocardium were measured in the short axis slice with the highest level of enhancement. The cut off signal intensity value for LGE was two times the standard deviation of remote unenhanced myocardium. The absolute and relative amounts of LV LGE were computed by the post-processing software. RV LGE was considered to be present when seen in both the short axis and four chamber views. The distribution of LV LGE was classified as subendocardial, mid-wall, sub-epicardial, patchy three-layer involvement, or confluent transmural involvement. Two blinded, independent observers (RT, BMM) were asked to differentiate between the three groups based on the presence and distribution of LGE. To test for intraobserver variability, one observer (RT) repeated the assessment after a month, while blinded to previous results.

\section{Statistical analysis}

All statistical analyses were performed with a commercially available statistical software program (SSPS for Windows, version 21; SPSS, Chicago, Ill). Group data were expressed as mean $\pm \mathrm{SD}$. Continuous variables were assessed using the parametric $t$ test for independent samples or Mann Whitney test where appropriate, and all categorical variables were as- 
sessed using the chi-square test. Statistical significance was defined as a $\mathrm{p}$ value less than 0.05. Bland-Altman analyses and intra-class correlation coefficients were used to determine intra-observer variability in the assessment of ventricular masses, volumes, ejection fractions, and the amount of LGE. We used kappa values to assess interobserver variability in determining the presence and localization of LGE, the diagnostic accuracy of the observers and the intra-observer variability in diagnosing CS and infarcts by LGE CMR.

\subsection{Results}

\section{Patient characteristics}

The demographic and background medical data are summarized in Table 1. The patients suffering from coronary artery disease were significantly older, had more cardiovascular risk factors, and pathological Q-waves on their 12-lead ECG's.

\section{Cardiac Magnetic Resonance}

The CMR findings are summarized in Table 2. The LV ejection fraction and LV enddiastolic volume index differed significantly between the CS and infarct groups, with more loss of regional LV wall thickness $(\mathrm{P}=0.009)$ and wall motion abnormalities $(\mathrm{P}=0.008)$ resulting in poorer systolic function $(\mathrm{P}=0.047)$ and larger ventricles $(\mathrm{P}=0.01)$ in the latter group. LGE of the LV was present in 29/30 CS patients (mean 3.8 segments/patient, range $0-12$ ), 30/30 infarct patients (mean 4.3 segments/patient, range $0-9$ ), and none of the control group. The amount of LGE did not significantly differ between the CS and infarct groups $(\mathrm{P}=0.8)$. Most enhancing lesions in the CS group were located in the basal and anterolateral LV (segments 1-6, 12 and 16; 74 out of 117 enhancing segments (64\%), compared to $58 / 127$ (46\%) for the MI group). In the majority of CS patients $(19 / 30,63 \%)$ patchy LGE involved all three myocardial layers. In 2/30 (7\%) confluent transmural LGE was present, suggesting co-existing coronary artery disease. (Figure 1A) But coronary angiography showed unobstructed epicardial coronary arteries. In 25/30 (83\%) patients of the infarct group the distribution of LGE was suggestive of underlying coronary artery disease, since hyper-enhancement started at the subendocardium and involved segments restricted to the vascular territory of specific coronary arteries. Sole mid-layer involvement was only seen in patients with CS, while LGE involving both the subendocardial and mid myocardial layers was predominantly seen in infarct patients. When considering sole mid layer, patchy subendocardial and three layer LGE to be diagnostic of CS, 26/30 (87\%) would have been correctly classified. (Figure 2A, C, E) Significantly more RV LGE (8 $(27 \%)$ versus $3(7 \%)$ patients, $\mathrm{P}=0.01)$ and $\mathrm{RV}$ dilation $(10(33 \%)$ versus $2(7 \%)$ patients, $\mathrm{P}=0.02$ ) was found in the CS group. (Figure 3) The observers correctly diagnosed CS in $21 / 29(72 \%), 24 / 29(83 \%), 21 / 29$ (72\%), ischemic heart disease in 23/30 (77\%), 23/30 (77\%), 24/30 (80\%) and normal controls in $9 / 10(90 \%), 10 / 10(100 \%), 10 / 10(100 \%)$. The kappa values for interobserver agreement in differentiating between CS, infarcts and normal controls by assessing the LGE CMR studies were $0.91(0.86,0.95)$, and $0.86(0.81$, 
0.92). The kappa value for intra-observer agreement in differentiating between CS, infarcts and normal controls by assessing the LGE CMR studies was $0.86(0.81,0.92)$. There was excellent intra- and interobserver correlation for ventricular volumes, masses, and ejection fractions. The intra-class correlation coefficient for LV LGE was 0.989 [0.981-0.993] $(\mathrm{p}=0.001)$.

\subsection{Discussion}

Our study is the first to systematically compare the distribution of LGE in CS with the findings in patients with coronary artery disease. Our findings suggest that CECMR is helpful in the non-invasive differentiation between patients with CS and patients with coronary artery disease and previous myocardial infarcts. However, in approximately a third of patients with coronary artery disease, subepicardial or patchy, three layer LGE was found, a pattern that was suggestive of a non-ischemic etiology such as CS. $(6,7,8)$ Additional angiographic information was needed to correctly classify 11 patients with coronary artery disease and 3 with CS.

LGE results from the sustained presence of gadolinium in the myocardium, secondary to expansion of the interstitial space or impaired microvascular wash out ("no-reflow phenomena"), and has been reported in a variety of conditions, such as coronary artery disease, cardiomyopathies, myocarditis, and myocardial infiltration, that are characterized by necrosis, fibrosis or inflammation. (2-9) In CS the presence of active, granulomatous inflammation and the resulting myocardial fibrosis are considered to be the underlying histopathological substrate resulting in the accumulation of gadolinium. (2-4) Recent studies have determined subendocardial and transmural LGE to be diagnostic of underlying coronary artery disease, while sole mid-layer and epicardial LGE were predominantly seen in patients with non-ischemic cardiomyopathies. (2-9) Although sole subepicardial myocardial fibrosis is considered rare in coronary artery disease, both our patients with epicardial LGE happened to be infarct patients. (13) (Figure 3) Early presentation and primary percutaneous interventions may have resulted in smaller, myocardial scars with an atypical mural distribution. (14) (Figure 2B, D, F, 4B, D)

The pattern of LGE in CS has been reported to be patchy, multi-segmental, not related to coronary artery territories, while predominantly involving the mid myocardial and subepicardial layers. (2-4). When considering sole mid-layer, and patchy subendocardial or patchy transmural LGE to be diagnostic of CS, and confluent subendocardial or confluent transmural LGE diagnostic of coronary artery disease, the sensitivity, specificity and overall accuracy of LGE CMR for these conditions respectively would be $87 \%, 70 \%, 78 \%$, and $63 \%, 90 \%$ and $77 \%$. The significantly higher number of patients with loss of regional wall thickness, wall motion abnormalities, and generally poorer systolic LV function in the infarct group is explained by more extensive, confluent, transmural LGE in this group. (15)

RV LGE and dilation was present in a substantial number of CS patients. These findings may be explained by primary RV myocardial involvement, but alternatively the presence of pulmonary arterial hypertension, resulting from extensive pulmonary sarcoidosis, has been considered a possible cause of RV fibrosis. (16) The junction of right and left 
ventricle and right-sided interventricular septum seemed to be predominantly affected. (Figure 4) However, further studies are needed to elucidate the underlying mechanisms and significance of RV LGE in patients with sarcoidosis. The absense of LGE in one patients with CS, with a dilated, globally hypokinetic LV, may be explained by the presence of diffuse myocardial fibrosis, which is not detected with the IR-GRE technique.

IR-GRE is the current gold standard technique in the assessment of myocardial fibrosis, and we may have underestimated the amount of myocardial scar tissue by relying on Spin Echo sequences in 5 patients. The distribution of LGE in these particular patients was however strongly suggestive of CS, in that it concerned patchy subendocardial or three layer hyperenhancement. Since the majority of patients were not assessed with first-pass myocardial perfusion studies, we did not include these data. The presence of flow limiting coronary artery disease in the infarct group might have improved the diagnostic accuracy of CMR in this group.

\subsection{Conclusion}

The presence of sole mid-layer, and patchy, subendocardial or three layer LGE with a nonvascular distribution, in patients with extra-cardiac sarcoidosis suggests cardiac involvement, and differentiates these patients from patients with coronary artery disease. 


\section{References}

1. Kim JS, Judson MA, Donnino R, et al. Cardiac sarcoidosis. Am Heart J. 2009;157(1):9-21.

2. Smedema JP, Snoep G, van Kroonenburgh MJPG, et al. Evaluation of the diagnostic accuracy of gadolinium CMR in cardiac sarcoidosis. J Am Coll Cardiol 2005;45:1683-1690.

3. Vignaux O, Dhôte R, Duboc D, et al. Clinical significance of myocardial magnetic resonance abnormalities in patients with sarcoidosis. A 1-year follow-up study. Chest 2002;122:1895-1901.

4. Vignaux O, Dhôte R, Duboc D, et al. Detection of myocardial involvement in patients with sarcoidosis applying T2-weighted, contrast-enhanced and cine magnetic resonance imaging: initial results of a prospective study. J Comput Assist Tomograph 2002;26:762-7.

5. Pennell DJ, Sechtem UP, Higgins CB, et al. Special article. Clinical indications for cardiovascular magnetic resonance (CMR): Consensus Panel report. European Heart Journal 2004;25:1940-1965.

6. Hunold P, Schlosser T, Vogt FM, et al. Myocardial late enhancement in contrast-enhanced cardiac MRI: distinction between infarction scar and non-infarction-related disease. Am J Roentgenol 2005;184(5):1420-6.

7. Lim RP, Srichai MB, Lee VS. Non-Ischemic Causes of delayed myocardial hyper-enhancement on MRI. Am J Roentgenol 2007; 188:1675-1681

8. Mahrholdt H, Wagner A, Judd RM, Sechtem U, Kim RJ. Delayed enhancement cardiovascular magnetic resonance assessment of non-ischemic cardiomyopathies. Eur Heart J 2005;26:1461-1474.

9. Laissy JP, Hyafil F, Feldman LJ, et al. M. Differentiating acute myocardial infarction from myocarditis: diagnostic value of early- and delayed-perfusion cardiac MR imaging. Radiology 2005;237:75-82.

10. Myocardial infarction redefined - A consensus document of the joint European Society of Cardiology/American College of Cardiology Committee for the Redefinition of Myocardial Infarction. J Am Coll Cardiol 2000;36:959-969.

11. Bellenger NG, Pennell DJ. Ventricular function. In: Manning WJ, Pennell DJ, eds. Cardiovascular Magnetic Resonance. New York, NY: Churchill Livingstone; 2002.

12. Cerqueira MD, Weissman NJ, Dilsizian V, et al. AHA writing group on myocardial segmentation and registration for cardiac imaging. Standardized myocardial segmentation and nomenclature for tomographic imaging of the heart. Circulation 2002;105:539-542.

13. Shirani J, Roberts WC. Subepicardial myocardial lesions. Am Heart J 1993;125:1346-1352.

14. Ricciardi MJ, Wu E, Davidson CJ, et al. Visualization of discrete microinfarction after percutaneous coronary intervention associated with mild creatine kinase-MB elevation. Circulation 2005;111:1027-1032.

15. Kim RJ, Wu E, Rafael A, et al. The use of contrast-enhanced magnetic resonance imaging to identify reversible myocardial dysfunction. N Engl J Med 2000;343:1445-1453.

16. McCann GP, Gan CT, Beek Am, et al. Extent of MRI Delayed Enhancement of Myocardial Mass Is Related to Right Ventricular Dysfunction in Pulmonary Artery Hypertension. AJR 2007; 188:349-35 
Table 1: Summary of demographic and background medical data.

\begin{tabular}{|c|c|c|c|c|}
\hline & $\begin{array}{l}\text { Coronary Artery Disease } \\
(\mathrm{n}=30)\end{array}$ & $\begin{array}{l}\text { Cardiac Sarcoidosis } \\
(\mathrm{n}=30)\end{array}$ & $\begin{array}{l}\text { Controls } \\
(\mathrm{n}=10)\end{array}$ & $\mathrm{p}$ value* \\
\hline Mean age (years) & $62 \pm 13$ & $49 \pm 8$ & $39 \pm 14$ & $\mathrm{p}<0.001$ \\
\hline $\operatorname{Sex}(M / F)$ & $27 / 3$ & $19 / 11$ & $9 / 1$ & $\mathrm{p}=0.02$ \\
\hline Body Surface Area $\left(\mathrm{m}^{2}\right)$ & $1.93 \pm 0.11$ & $1.89 \pm 0.19$ & $1.89 \pm 0.22$ & $\mathrm{p}=0.3$ \\
\hline $\begin{array}{l}\text { Functional class } \\
\text { NYHA I } \\
\text { NYHA II } \\
\text { NYHA III } \\
\text { NYHA IV }\end{array}$ & $\begin{array}{r}10 \\
17 \\
3 \\
0\end{array}$ & $\begin{array}{r}14 \\
10 \\
4 \\
2\end{array}$ & $\begin{array}{r}10 \\
0 \\
0 \\
0\end{array}$ & $\mathrm{p}=0.2$ \\
\hline Angina & 30 & 0 & 0 & $\mathrm{p}<0.001$ \\
\hline Palpitations & 4 & 19 & 0 & $\mathrm{p}<0.001$ \\
\hline Syncope & 2 & 4 & 0 & $\mathrm{p}=0.7$ \\
\hline Hypertension & 10 & 2 & 0 & $\mathrm{p}=0.02$ \\
\hline Diabetes Mellitus & 8 & 1 & 0 & $\mathrm{p}=0.03$ \\
\hline $\begin{array}{l}\text { 12-lead ECG } \\
\text { Atrial Fibrillation }\end{array}$ & 0 & 0 & 0 & \\
\hline Bundle Branch Block & 5 & 7 & 0 & $\mathrm{p}=0.5$ \\
\hline $\mathrm{RBBB}$ & 0 & 1 & 0 & \\
\hline LBBB & 4 & 3 & 0 & \\
\hline LAHB & 1 & 1 & 0 & \\
\hline Bifascicular block & 0 & 2 & 0 & \\
\hline Atrio-ventricular Block & 0 & 4 & 0 & $\mathrm{p}=0.1$ \\
\hline Grade II AV Block & 0 & 2 & 0 & \\
\hline Grade III AV Block & 0 & 2 & 0 & \\
\hline Q wave & 11 & 2 & 0 & $\mathrm{p}=0.01$ \\
\hline
\end{tabular}

* P values concern the comparison between he groups with coronary artery disease and cardiac sarcoidosis 
Table 2: Summary of the findings with gadolinium-enhanced cardiac magnetic resonance.

\begin{tabular}{|c|c|c|c|c|}
\hline & $\begin{array}{l}\text { Coronary Artery } \\
\text { Disease }\end{array}$ & $\begin{array}{l}\text { Cardiac } \\
\text { Sarcoidosis }\end{array}$ & Controls & $\mathrm{P}$ value \\
\hline & $(\mathrm{n}=30)$ & $(\mathrm{n}=30)$ & $(n=10)$ & \\
\hline Left Ventricular mass (gram) & $122 \pm 28$ & $129 \pm 61$ & $97 \pm 40$ & $\mathrm{p}=0.6$ \\
\hline Left Ventricular mass index $\left(\mathrm{gram} / \mathrm{m}^{2}\right)$ & $64 \pm 16$ & $68 \pm 26$ & $51 \pm 18$ & $\mathrm{p}=0.5$ \\
\hline Left Ventricular Hypertrophy (pts) & $4(13 \%)$ & $7(23 \%)$ & 0 & $\mathrm{p}=0.5$ \\
\hline Regional LGE (pts) & $30(100 \%)$ & $29(97 \%)$ & 0 & $\mathrm{p}=1.0$ \\
\hline Number of Left Ventricular segments with LGE & $127 / 510(25 \%)$ & $117 / 510(23 \%)$ & 0 & $\mathrm{p}=0.4$ \\
\hline Range per patient & $0-9$ & $0-12$ & & \\
\hline Mean & 4.3 & 3.8 & & \\
\hline Sub-endocardial LGE (pts) & $6(20 \%)$ & $5(17 \%)$ & 0 & $\mathrm{p}=1.0$ \\
\hline Confluent, coronary artery distribution & $6(20 \%)$ & 0 & & $\mathrm{p}=0.02$ \\
\hline Patchy & 0 & $5(17 \%)$ & & $\mathrm{p}=0.01$ \\
\hline Sub-endocardial + Mid-wall LGE (pts) & $5(17 \%)$ & $1(3 \%)$ & 0 & $\mathrm{p}=0.2$ \\
\hline Mid-wall MDE (pts) & 0 & $2(7 \%)$ & 0 & $\mathrm{p}=0.5$ \\
\hline Sub-epicardial + Mid-wall LGE (pts) & $2(7 \%)$ & 0 & 0 & $\mathrm{p}=0.5$ \\
\hline Patchy LGE of all 3 Left Ventricular layers (pts) & $9(30 \%)$ & $19(63 \%)$ & 0 & $\mathrm{p}=0.01$ \\
\hline Transmural confluent Left Ventricular LGE (pts) & $8(27 \%)$ & $2(7 \%)$ & 0 & $\mathrm{p}=0.04$ \\
\hline One focus of LGE (pts) & 21 & 10 & 0 & $\mathrm{p}=0.02$ \\
\hline Multiple foci of LGE (pts) & 9 & 19 & 0 & $\mathrm{p}=0.02$ \\
\hline Vascular segmental distribution of LGE (pts) & 25 & 3 & 0 & $\mathrm{p}<0.001$ \\
\hline Left Ventricular LGE (gram) & & & 0 & $\mathrm{p}=0.8$ \\
\hline Min - Max & jul-68 & $0-73$ & & \\
\hline Mean & $26 \pm 14$ & $27 \pm 21$ & & \\
\hline Left Ventricular LGE (\%) & & & & $\mathrm{p}=0.8$ \\
\hline Min - Max & jun-47 & $0-38$ & & \\
\hline Mean & $19 \pm 11$ & $19 \pm 12$ & & \\
\hline Left Ventricular End-Diastolic Volume (ml) & & & & $\mathrm{p}=0.08$ \\
\hline Min - Max & $103-573$ & $74-308$ & $107-229$ & \\
\hline Mean \pm SD & $194 \pm 84$ & $160 \pm 58$ & $154 \pm 33$ & \\
\hline Left Ventricular End-Diastolic Volume index $\left(\mathrm{ml} / \mathrm{m}^{2}\right)$ & & & & $\mathrm{p}=0.01$ \\
\hline Min - Max & $57-311$ & $36-148$ & $64-102$ & \\
\hline Mean \pm SD & $101 \pm 47$ & $75 \pm 29$ & $81 \pm 101$ & \\
\hline Left Ventricular Ejection Fraction (\%) & & & & $\mathrm{p}=0.005$ \\
\hline Min - Max & jun-63 & $21-70$ & $52-73$ & \\
\hline Mean \pm SD & $37 \pm 14$ & $49 \pm 14$ & $62 \pm 6$ & \\
\hline Dilated Left Ventricle (pts) & $15(50 \%)$ & $9(30 \%)$ & $1(10 \%)$ & $\mathrm{p}=0.1$ \\
\hline Left Ventricular Ejection Fraction < 55\% (pts) & $25(83 \%)$ & $17(57 \%)$ & $1(10 \%)$ & $\mathrm{p}=0.047$ \\
\hline Loss of Left Ventricular wall thickness (pts) & $20(67 \%)$ & $9(30 \%)$ & 0 & $\mathrm{p}=0.009$ \\
\hline Left Ventricular wall motion abnormalities (pts) & $23(77 \%)$ & $12(40 \%)$ & 0 & $\mathrm{p}=0.008$ \\
\hline Right Ventricular LGE (pts) & $3(7 \%)$ & $8(27 \%)$ & 0 & $\mathrm{p}=0.01$ \\
\hline Dilated Right Ventricle (pts) & $2(7 \%)$ & $10(33 \%)$ & 0 & $\mathrm{p}=0.02$ \\
\hline Right Ventricular Ejection Fraction $<45 \%$ (pts) & $19(63 \%)$ & $12(40 \%)$ & $1(10 \%)$ & $\mathrm{p}=0.1$ \\
\hline Right Ventricular End-Diastolic Volume (ml) & & & & $\mathrm{P}=0.7$ \\
\hline Min - Max & $94-258$ & $79-260$ & $94-169$ & \\
\hline Mean $\pm \mathrm{SD}$ & $162 \pm 38$ & $157 \pm 48$ & $123 \pm 28$ & \\
\hline Right Ventricular End-Diastolic Volume index $\left(\mathrm{ml} / \mathrm{m}^{2}\right)$ & & & & $\mathrm{p}=0.9$ \\
\hline Min - Max & $53-147$ & $40-138$ & $55-87$ & \\
\hline Mean \pm SD & $85 \pm 20$ & $85 \pm 26$ & $65 \pm 10$ & \\
\hline Right Ventricular Ejection Fraction (\%) & & & & $\mathrm{p}=0.5$ \\
\hline Min - Max & $15-60$ & $18-64$ & $46-69$ & \\
\hline Mean \pm SD & $43 \pm 12$ & $46 \pm 12$ & $58 \pm 6$ & \\
\hline
\end{tabular}

* P values concern the comparison between the groups with coronary artery disease and cardiac sarcoidosis; LGE $=$ myocardial delayed enhancement 


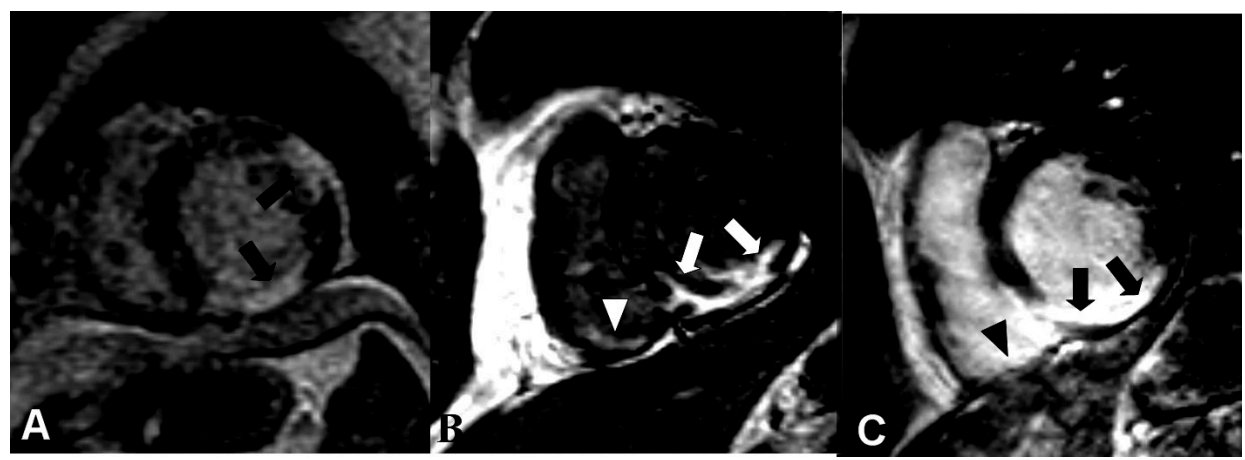

Figure 1. Delayed-enhanced three-dimensional breath-hold inversion-recovery gradient echo studies (short axis views) demonstrating multi-focal transmural hyper-enhancement involving the inferior and lateral LV segments in a patient with sarcoidosis (A), and patchy (B) respectively confluent (C) transmural inferior LV (arrows) and RV (arrowheads) wall late gadolinium enhancement secondary to infarctions in patients with coronary artery disease of the RCA. 


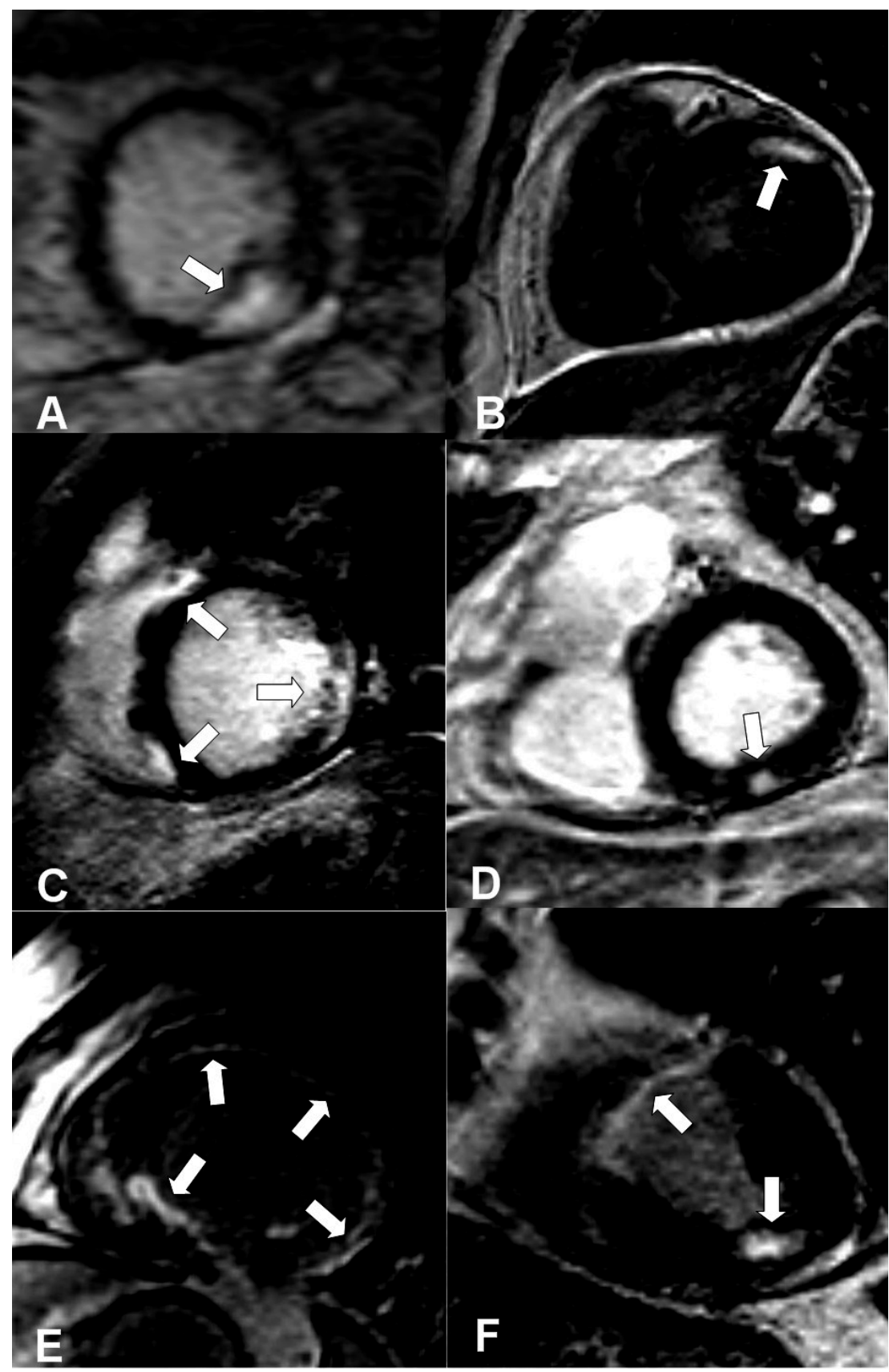

Figure 2. Delayed-enhanced three-dimensional breath-hold inversion-recovery gradient echo studies (short axis views) of three patients with cardiac sarcoidosis, demonstrating: focal (A) and patchy, multi-segmental three layer hyper-enhancement ( $\mathrm{C}$ and $\mathrm{E}$ ), and three patients with coronary artery disease demonstrating: focal sub-epicardial infarctions in the distribution of respectively the LCX (B) and RCA (D), and two-vessel disease (F) involving the LAD (subendocardial infarction) and RCA (mid-layer infarction). 


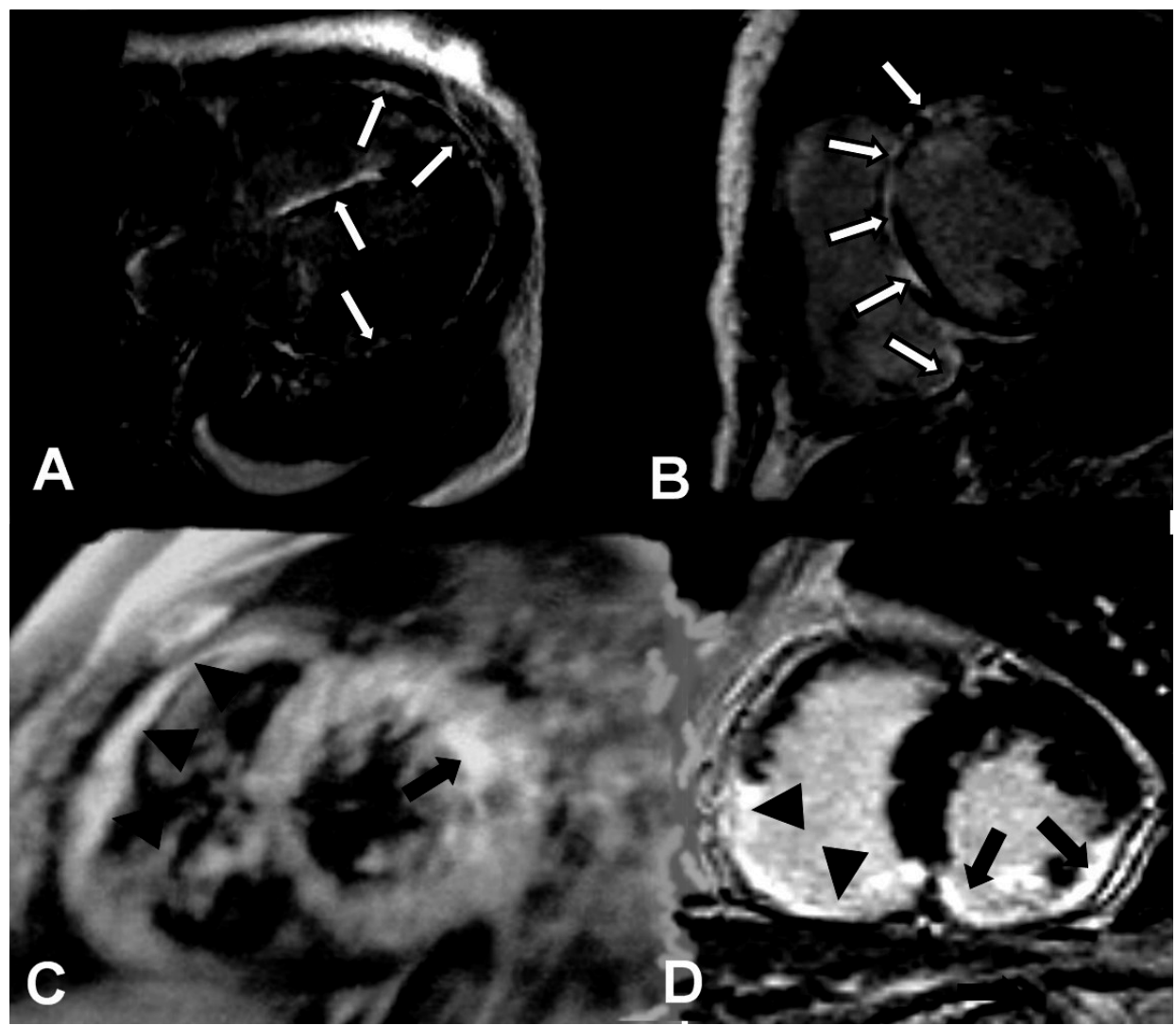

Figure 3. Delayed-enhanced three-dimensional breath-hold inversion-recovery gradient echo studies (A: 4 chamber view; B, D: short axis views) demonstrating multi-segmental, patchy three layer hyper-enhancement in a patients with sarcoidosis $(A, B)$ and confluent transmural hyper-enhancement in the vascular distribution of the RCA (D). The delayed-enhanced spin echo study (C: short axis view) shows patchy, three layer involvement of the lateral LV segments. Transmural right ventricular enhancement secondary to sarcoidosis (C) and coronary artery disease (D) is demonstrated by the arrowheads. 


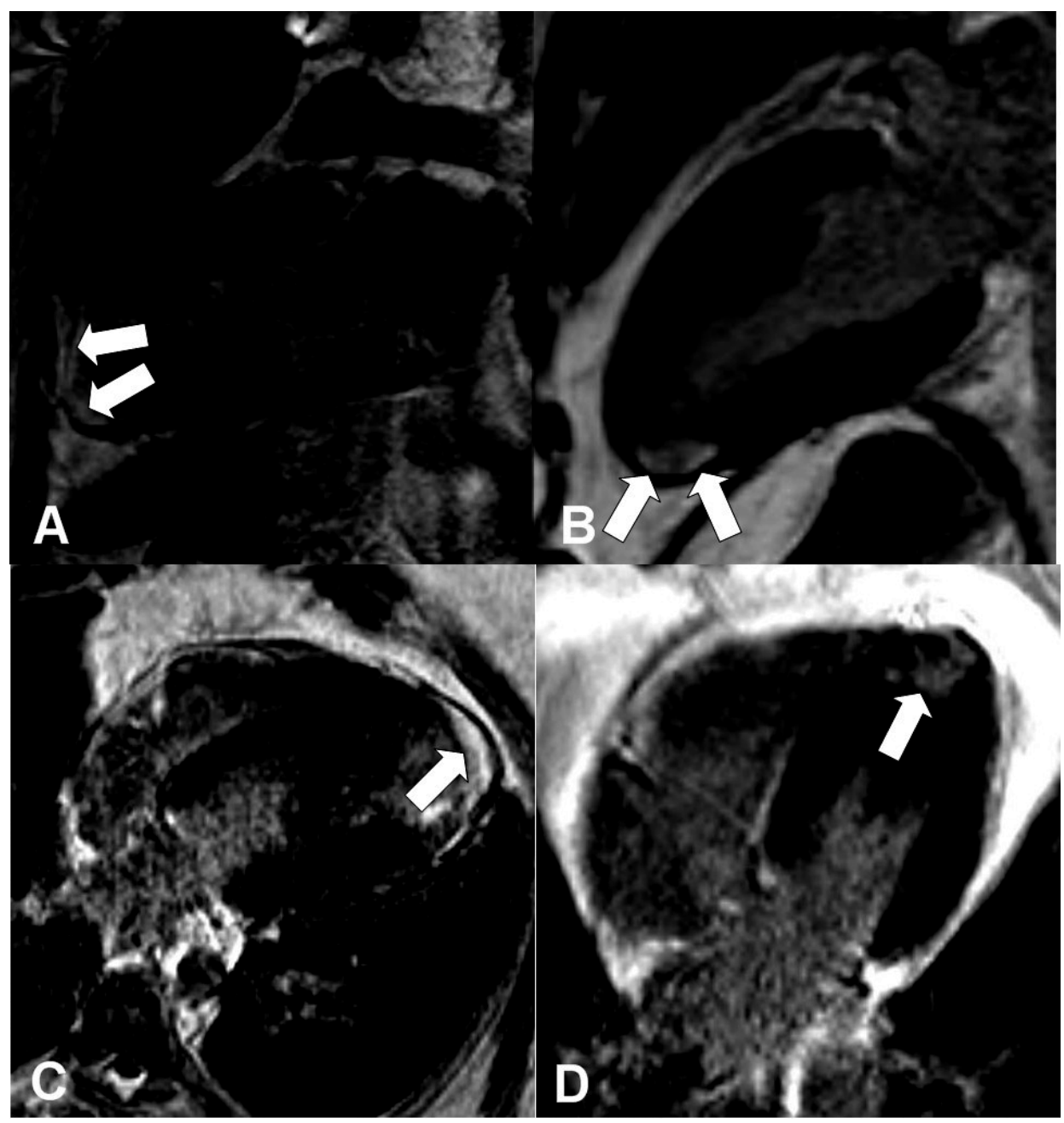

Figure 4. Delayed-enhanced three-dimensional breath-hold inversion-recovery gradient echo studies demonstrating confluent apical hyper-enhancement secondary to sarcoidosis (A: vertical long axis view, C: 4 chamber view) and apical patchy three-layer hyper-enhancement in a patient with coronary artery disease and myocardial infarction secondary to obstruction of the LAD (B: vertical long axis view, D: 4 chamber view). 


\section{Chapter 7}

\section{Right ventricular involvement in cardiac sarcoidosis demonstrated with cardiac magnetic resonance}

ESC Heart Failure 2017;4(4):535-544.

Jan-Peter Smedema ${ }^{1}, \mathrm{MD}$, Robert-Jan van Geuns ${ }^{2}, \mathrm{MD}, \mathrm{PhD}$, Gillian Ainslie ${ }^{3}, \mathrm{MB}$ ChB FRCP, Joris Ector ${ }^{4}, \mathrm{MD}$, PhD, Hein Heidbuchel $^{5}$, MD, PhD, Harry J.G.M.Crijns ${ }^{1}$, MD, $\mathrm{PhD}$.

${ }^{1}$ Department of Cardiology, Maastricht University Medical Centre, Maastricht, The Netherlands.

${ }^{2}$ Department of Cardiology, Erasmus Medical Centre, Rotterdam, The Netherlands.

${ }^{3}$ Respiratory Clinic, Department of Medicine, Groote Schuur Hospital, Cape Town, Republic of South Africa.

${ }^{4}$ Department of Cardiology, University Hospitals Gasthuisberg, Leuven, Belgium.

${ }^{5}$ University of Hasselt Heart Centre, Virga Jessa Hospital, Hasselt, Belgium. 


\begin{abstract}
Aims: Cardiac involvement in sarcoidosis is reported in up to $30 \%$ of patients. Left ventricular involvement demonstrated by contrast-enhanced cardiac magnetic resonance has been well validated. We sought to determine the prevalence and distribution of right ventricular late gadolinium enhancement in patients diagnosed with pulmonary sarcoidosis.
\end{abstract}

Methods and results: We prospectively evaluated 87 patients diagnosed with pulmonary sarcoidosis with contrast-enhanced cardiac magnetic resonance for right ventricular involvement. Pulmonary artery pressures were non-invasively evaluated with Doppler echocardiography. Patient characteristics were compared between the groups with and without right ventricular involvement, and right ventricular enhancement was correlated with pulmonary hypertension, ventricular mass, volume, and systolic function. Left ventricular late gadolinium enhancement was demonstrated in 30 patients $(34 \%)$. Fourteen patients $(16 \%)$ had right ventricular late gadolinium enhancement, with sole right ventricular enhancement in only two patients. The pattern of right ventricular enhancement consisted of right ventricular outflow tract enhancement in 1 patient, free wall enhancement in 8 patients, ventricular insertion point enhancement in 10 patients, and enhancement of the right side of the interventricular septum in 11 patients. Pulmonary arterial hypertension correlated with the presence of right ventricular enhancement $(p<0.001)$. Right ventricular enhancement correlated with systolic ventricular dysfunction $(p<0.001)$, hypertrophy $(p=0.001)$, and dilation $(\mathrm{p}<0.001)$.

Conclusion: Right ventricular enhancement was present in $16 \%$ of patients diagnosed with pulmonary sarcoidosis, and in $48 \%$ of patients with left ventricular enhancement. The presence of right ventricular enhancement correlated with pulmonary arterial hypertension, right ventricular systolic dysfunction, hypertrophy and dilation.

\title{
Contents
}

Abstract

7.1 Introduction

7.2 Methods

7.3 Results

7.4 Discussion

7.5 Conclusions

7.6 Limitations

7.7 Future focus of development

7.8 Acknowledgements

References
Error! Bookmark not defined. Error! Bookmark not defined. Error! Bookmark not defined. Error! Bookmark not defined. Error! Bookmark not defined. Error! Bookmark not defined. Error! Bookmark not defined. Error! Bookmark not defined. Error! Bookmark not defined. Error! Bookmark not defined. 


\subsection{Introduction}

Sarcoidosis is a rare, inflammatory condition, resulting from an uncontrolled cellular inflammatory response in genetically predisposed individuals, which affects the heart in approximately a third of patients. (1) Left ventricular involvement demonstrated by contrastenhanced cardiac magnetic resonance has been well validated. (2) Until recently limited attention has been given to right ventricular involvement in cardiac sarcoidosis, its prevalence, relevance and prognostic value. (3) Cardiac magnetic resonance imaging is the preferred imaging tool to evaluate the healthy and diseased right ventricle. $(4,5)$ Right ventricular volumes, mass and function can be quantified without geometric assumptions and excellent intra- and inter-observer agreement and inter-study reproducibility. $(4,5,6)$ Delayed contrast-enhanced magnetic resonance allows for the detection and quantification of focal scar and interstitial fibrosis. Although there are numerous reports on delayed contrast-enhanced cardiac magnetic resonance delineating left ventricular sarcoidosis, relatively few studies have reported on right ventricular involvement. $(3,7,8,9,10)$ We sought to determine the prevalence and distribution of right ventricular late gadolinium enhancement in patients diagnosed with pulmonary sarcoidosis, and determine the relationship with pulmonary hypertension, ventricular volume, mass and systolic function.

\subsection{Methods}

\section{Patient selection}

Between July 2001 and March 2014, we enrolled 87 consecutive patients with histologically proven pulmonary sarcoidosis. Cardiac evaluation was performed because of symptoms or routine screening to exclude cardiac involvement. Patients were excluded when the standard contra-indications for contrast-enhanced cardiac magnetic resonance existed. Institutional Review Board approval was obtained for this study.

\section{Baseline investigations}

Baseline investigations included 12-lead electrocardiography, Doppler echocardiography, and contrast-enhanced cardiac magnetic resonance. Pulmonary artery systolic pressure was estimated from the tricuspid regurgitant velocity plus an estimate of right atrial pressure derived from the inferior vena cava. (11) Right-sided heart studies (26) were performed in patients with pulmonary hypertension, congestive heart failure and in patients in whom automated cardioverter defibrillators were implanted. Coronary angiography was performed to exclude underlying coronary artery disease in patients with documented ventricular tachy-arrhythmias, pathological Q-waves, impaired systolic function, regional wall motion abnormalities, and/or late gadolinium enhancement. 


\section{CMR protocol and analysis}

Studies were performed using a commercial 1.5Tesla scanner with a cardiac-dedicated phased-array coil. The cardiac magnetic resonance studies were electrocardiographically triggered by standard software. Studies consisted of multi-phase multi-slice steady-state-free precession, and fat-saturated T2-weighted (69) breath-hold sequences of the short axis, vertical long axis and horizontal long axis views. Outflow tract views were generated in patients with right ventricular abnormalities. The short-axis images covered the left ventricle from base to apex. The steady-state-free precession sequences (typical repetition time: $3.5 \mathrm{msec}$; echo-time $1.4 \mathrm{msec}$; flip-angle $55^{\circ}$, temporal resolution $50 \mathrm{msec}$, voxel size $1.6 \times 1.6 \times 10 \mathrm{~mm}$, no gap) were performed to assess regional wall-motion abnormalities, left and right ventricular masses, volumes and ejection fractions. Papillary muscles were included when determining right ventricular mass, and excluded when determining volumes. Contrast-enhanced and T2 weighted images were obtained in diastole to minimize artifact due to cardiac motion. Ten minutes after the additional administration of $0.1 \mathrm{mmol} / \mathrm{kg}$ gadolinium-diethylenetriaminepenta-acetic acid. (Schering, Berlin, Germany), a twodimensional segmented inversion recovery-gradient echo breath-hold sequence (short axis, vertical long axis, horizontal long axis, and right ventricular outflow tract in selected patients, voxel size $1.6 \times 1.6 \times 10 \mathrm{~mm}$, without gap), was used to assess for late gadolinium enhancement. The inversion time (250 to $400 \mathrm{msec}$ ) was determined on an individual basis to obtain optimal nulling of the unenhanced myocardial signal. Two experienced, blinded, and independent observers used commercially available software (CAAS MRV 3.4, Pie Medical Imaging, Maastricht, The Netherlands) to determine the standard parameters delineated in Table 1. The distribution of right ventricular late gadolinium enhancement was determined by consensus and characterized as free wall, apical, outflow tract, right-sided interventricular septal, and/or including the ventricular insertion points. Late ventricular gadolinium enhancement was considered present only if confirmed on both short-axis and matching long-axis myocardial locations. Late left ventricular gadolinium enhancement was quantified by a semiautomatic detection method using the signal intensity threshold of $\geq 2$ SD above a remote reference region. The intra- and interobserver variability's were determined by calculating the variability coefficients and intra-class correlations for each parameter in 18 randomly selected studies.

\section{Variables and definitions}

Peak systolic right ventricular pressures over $35 \mathrm{~mm} \mathrm{Hg}$ were considered to represent pulmonary hypertension. Right ventricular hypertrophy was defined as right ventricular weight exceeding normal values as published by Maceira et al and/or right ventricular end-diastolic wall thickness over 5 millimeters. (6) Right ventricular systolic dysfunction was defined as an ejection fraction below $45 \%$. (12) 


\section{Statistical analysis}

All statistical analyses were performed using statistical software (Version 21.0, SPSS; Chicago, IL). Continuous normal distributed variables were expressed as mean $\pm S D$, and between group comparisons were made using the parametric $t$ test for independent samples or the Mann-Whitney test when appropriate. In the non-normally distributed continuous data the median and interquartile range were determined, and between group correlations were made with the Wilcoxon test. Categorical variables were assessed using the chi-square or Fischer Exact test when appropriate. Statistical significance was defined at $\mathrm{p}<0.05$.

\subsection{Results}

\section{Patient characteristics}

Table 1 demonstrates the baseline characteristics in the included 87 patients. Twenty-seven (31\%) patients presented with cardiac symptoms, while the remaining 60 either suffered from non-specific symptoms or were routinely screened for cardiac sarcoidosis. According to the ACC/AHA/HRS 2008 guidelines for device-based therapy of cardiac rhythm abnormalities, an implantable cardioverter defibrillator or pacemaker was implanted in respectively 14 and 3 patients after the baseline cardiac magnetic resonance study. (13) All had late gadolinium enhancement and suffered of cardiac symptoms. When applying the recently published Heart Rhythm Society expert consensus criteria we diagnosed 31 patients (36\%) with cardiac involvement. (14)

\section{CMR analyses}

Thirty patients (34\%) had left ventricular and 14 patients (16\%) right ventricular enhancement, with enhancement limited to the right ventricle in only 2 patients $(2 \%)$. The group of patients with right ventricular enhancement had significantly more extensive left ventricular enhancement compared to those without. (Table 1, p $=0.007$ ) Table 2 describes the distribution of right ventricular enhancement. Right ventricular enhancement correlated with early-diastolic left-sided septal displacement $(\mathrm{p}=0.002)$, systolic right ventricular dysfunction $(\mathrm{p}<0.001)$, hypertrophy $(\mathrm{p}<0.001)$, and dilation $(\mathrm{p}=0.009)$. Fifteen patients had pulmonary hypertension, 8 of which had right ventricular enhancement. The presence of ventricular insertion point enhancement $(\mathrm{p}=0.007)$, right-sided septal $(\mathrm{p}<0.001)$ and right ventricular free wall enhancement $(\mathrm{p}=0.016)$ correlated with the presence of pulmonary hypertension. Right ventricular hypertrophy correlated with the presence of ventricular insertion point enhancement $(p=0.004)$ and right-sided septal $(p=0.001)$, but not with free wall enhancement $(\mathrm{p}=0.076)$. In 5 patients early-diastolic septal displacement towards the left ventricle was observed, 2 of which had insertion point enhancement $(\mathrm{p}=0.099)$, and 3 of which had right-sided septal enhancement $(\mathrm{p}=0.013)$. Neither the chronicity of pulmonary sarcoidosis, as determined by the time since diagnosis, nor the extent of lung disease as determined by high-resolution computed tomography, correlated with pulmonary hyper- 
tension, right ventricular systolic function or myocardial enhancement. The right ventricular end-diastolic volume index in patients with pulmonary Stage 4 (fibro-cystic disease), was significantly larger compared to the earlier disease Stage 1 (hilar nodes only) ( $p=0.027$ ). The T2-weighted studies suggested active, granulomatous disease in only 10/69 (14\%) of patients. The intraobserver variability for right ventricular end-diastolic volume was $2 \%$, end-systolic volume 3.4\%, ejection fraction 3.4\%, and mass $4 \%$. The interobserver variability for right ventricular end-diastolic volume was $1.6 \%$, end-systolic volume $\%$, ejection fraction $3.2 \%$, and mass $\%$.

\subsection{Discussion}

This is the first prospective cardiac magnetic resonance study to specifically report on the prevalence, and distribution of right ventricular involvement in cardiac sarcoidosis. Predominant or isolated right ventricular involvement is rare, with nearly all patients suffering from left ventricular disease. (15) With Murtagh et al, Crawford et al, and Cheong et al we found more extensive left ventricular enhancement to be associated with right ventricular involvement. $(\mathbf{8 , 1 0 , 1 6 )}$ Previous studies reported a correlation between the presence and extent of left ventricular enhancement, impaired left and right ventricular systolic function, and higher adverse event rates. The direct relationship between right ventricular enhancement, its size and systolic function, as previously demonstrated in left ventricular sarcoidosis, has not been reported before.

Right ventricular enhancement, inflammation and impaired systolic function have been associated with adverse outcome, particularly ventricular tachy-arrhythmias. $(\mathbf{1 0}, \mathbf{1 6}, \mathbf{1 7}, \mathbf{1 8}, \mathbf{1 9})$ However, the localizations of the arrhythmogenic foci were not reported on. Since right ventricular sarcoidosis occurs in patients with more extensive left ventricular disease, the reported prognostic relevance of right ventricular disease may at least partly reflect the extent of left ventricular arrhythmogenic substrate.

Several post-transplant and post-mortem studies in sarcoidosis patients have reported right ventricular involvement to range from $6 \%$, in patients dying from alternate causes, to as high as $65 \%$ in those dying sudden cardiac deaths. (20-24) Generally, patients with congestive failure were found to have extensive biventricular sarcoid (Figure 1), and in those who had died suddenly, active granulomatous infiltration and patchy scar were present. Right ventricular outflow tract involvement was rare. (Figure 2)

Ten recent studies employing contrast-enhanced magnetic resonance in the assessment of cardiac sarcoidosis, reported on right ventricular disease in sarcoidosis. (Table 3) $((3,7,8,9,10,16,17,18,19,25)$ Our findings in an unselected population with pulmonary sarcoidosis compare. Right ventricular enhancement was reported in 5 of these studies, and ranged from $2 \%$ in unselected to $48 \%$ in high-risk populations, which compares to our $47 \%$ of patients with left ventricular sarcoidosis. $(3,7,8,9,10)$ Similar to our findings, nearly all patients with right ventricular enhancement had left ventricular enhancement. Samar et al, Patel et al and Crawford et al were the only to report on right ventricular enhancement in more detail. $(3,7,10)$ Contrary to our and other studies, Samar et al reported that right ventricular enhancement did not correlate with a difference in left ventricular ejection 
fraction. His data were included in a poster presentation, which precluded detail, while relevant data were not available in $19 \%$ of patients. (3) Crawford reported multi-focal right ventricular enhancement in nearly half of those with left ventricular enhancement, basal, mid or apical right ventricular segments being equally involved. (10) Similar to Crawford et al, the large majority of our patients $(70 \%)$ had multi-focal right ventricular enhancement. However, most of our patients had right-sided septal enhancement, similar to Patel's findings. (7) Right-sided septal and insertion point enhancement were related to pulmonary hypertension in 8 of our patients. Crawford et al and Patel et al did not include data on right ventricular or pulmonary pressures. Compared to Crawford's population, our patients with right ventricular enhancement had on average poorer systolic function, and more extensive left ventricular enhancement.

The presence of right ventricular enhancement correlated with hypertrophy, dilation and systolic dysfunction. The relationship between pulmonary hypertension, right ventricular hypertrophy, dilation, systolic dysfunction, septal displacement and septal and insertion point enhancement has been demonstrated before. (26) (Figure 3) Right ventricular enhancement in sarcoidosis may be caused by direct granulomatous infiltration, but also be related to pulmonary hypertension. (26) (Figures 1 and 3) Right ventricular hypertrophy, dilation, dysfunction and enhancement may be secondary to pulmonary hypertension, and are associated with worse prognosis. $(26,27)$ Pulmonary hypertension is found in $6-28 \%$ of the general sarcoidosis outpatient setting, and may be secondary to pulmonary fibrosis, angiitis and/or congestive heart failure. (27). The fact that pulmonary hypertension in our population did not correlate with disease extent as determined by computed tomography, illustrates the variety in pathophysiology of this condition in sarcoidosis. (28) Right ventricular dilation, systolic impairment, and inflammation, as demonstrated with positron emission tomography, in sarcoidosis have been demonstrated to predict adverse outcome in sarcoidosis. $(\mathbf{1 0}, \mathbf{1 2 , 1 6 , 1 7 , 1 8 , 2 5 , 2 9 )}$

In patients with predominant right ventricular disease, cardiac sarcoidosis needs to be differentiated from arrhythmogenic right ventricular cardiomyopathy. Distinguishing features favoring sarcoidosis consist of an older age of onset, a non-familiar pattern, wider QRS complexes, septal involvement with atrio-ventricular conduction disease, multiple arrhythmogenic foci, particularly right ventricular apical tachycardia, concomitant left ventricular disease, and the presence of mediastinal lymphadenopathy. (30) Electro-anatomic mapping, contrast-enhanced magnetic resonance and/or positron emission tomography may guide endomyocardial biopsies needed to obtain histological confirmation of the diagnosis.

We report on ventricular insertion point enhancement, a distribution pattern not specifically mentioned in the other studies. Late enhancement of the septum and insertion points may result from delayed wash-out of gadolinium due to altered myocardial fiber strain, fiber disarray, ischemia, and fibrosis, secondary to right ventricular pressure or volume overload, and resulting septal shift. $(\mathbf{2 6 , 3 2 , 3 3 )}$ Ventricular insertion point enhancement has been reported in hypertrophic cardiomyopathy, atrial septal defects, severe pulmonary hypertension, tetralogy of Fallot, transposition of the great arteries and even a proportion of veteran healthy endurance athletes. (31,32,33,34,35) (Figures 2, 3, 4 and 5) The amount of insertion point enhancement correlates with mean pulmonary arterial pressures, 
right ventricular mass, volume and ejection fraction. (26) Pulmonary hypertension in sarcoidosis is associated with adverse outcome, particularly when accompanied by right ventricular dysfunction, and/or lung fibrosis. $(26,27)$ Recently Swift et al reported septal extension of insertion point enhancement in pulmonary hypertension to mark more severe disease, with associated right ventricular dilation, but found it not to be an independent predictor of overall mortality. (26)

Late gadolinium enhancement includes active, potentially reversible, granulomatous inflammation as well chronic focal scar. $(\mathbf{8 , 1 9 , 3 6 )}$ Immune suppressive treatment, currently a work in progress, may potentially improve systolic function and decrease arrhythmogenic substrate. $(\mathbf{1 0 , 1 9 , 3 6 )}$ The T2-weigthed Spin Echo-based assessment used in our study to evaluate for active granulomatous infiltration and associated edema is rather insensitive. (8) T2 mapping and positron emission tomography have shown promise, and will be included in future projects. $(28,37)$

\subsection{Conclusions}

Approximately $30 \%$ of an unselected patient population with pulmonary sarcoidosis had left ventricular involvement, half of which had right ventricular involvement. More extensive left ventricular enhancement correlated with right ventricular involvement. Right ventricular enhancement may result from direct infiltration and resulting scar, or pulmonary hypertension. Previous studies associated impaired systolic right ventricular function and right ventricular enhancement with ventricular tachy-arrhythmias. We demonstrate right ventricular enhancement with cardiac magnetic resonance to be mostly multi-focal, involve the septum and correlate with increased right ventricular volumes, hypertrophy and impaired systolic function.

\subsection{Limitations}

Pulmonary pressures were routinely determined non-invasively in the majority of our patients, and we may have under-estimated the pulmonary pressures. Our imaging protocol was not primarily adapted to evaluate the right ventricle, our slice thickness, and potentially sub-optimal myocardial nulling may have resulted in an underestimation of the presence and extent of right ventricular infiltration and scarring. Since T2 mapping was not performed, we likely underestimated active granulomatous inflammation. The relatively small number of patients included limits our findings and conclusions.

\subsection{Future focus of development}

Customized hybrid approaches including electrocardiography, ultrasound, positron emission tomography and contrast-enhanced magnetic resonance will provide us with more 
sensitive, accurate and comprehensive information on hemodynamic, electrical, mechanical, and inflammatory characteristics of the atriae and ventricles.

\subsection{Acknowledgements}

The expert statistical advice of Dr. P. Nelemans, MD, PhD, of the Department of Epidemiology at Maastricht University Medical Centre is greatly valued.

\section{Conflicts of interest}

None declared. 


\section{References}

1. Youssef G, Beanlands RSB, Birnie DH, Nery PB. Cardiac sarcoidosis: applications of imaging in diagnosis and directing treatment. Heart 2011;97:2078-87.

2. Smedema JP, Snoep G, van Kroonenburgh MPG, van Geuns RJ, Dassen WRM, Gorgels APM, Crijns HJGM. Evaluation of the accuracy of gadolinium-enhanced cardiovascular magnetic resonance in the diagnosis of cardiac sarcoidosis J Am Coll Cardiol 2005;45:1683-90.

3. Samar HY, Thompson DV, Doyle M, Williams RB, Yamrozik JA, Reddy ST, Shah M, Biederman RJ. Sarcoidosis; is it confined to just the LV? An RV LGE study. From 17th Annual SCMR Scientific Sessions, New Orleans, LA, USA. 16-19 January 2014.

4. Valsangiocomo Buechel ER, Mertens L. Imaging the right heart: the use of integrated multimodality imaging. Eur Heart J 2012;33:949-60.

5. van de Veerdonck MC, Marcus JT, Bogaard HJ, Vonk Noordegraaf A. State of the art: advanced imaging of the right ventricle and pulmonary circulation in humans (2013 Grover Conference series). Pulm Circ 2014;4(2):158-68.

6. Maceira AM, Prasad SK, Khan M, Pennell DJ. Reference right ventricular systolic and diastolic function normalized to age, gender and body surface area from steady-state free precession cardiovascular magnetic resonance. Eur Heart J 2006;27:2879-88.

7. Patel MR, Cawley PJ, Heitner JF, Klem I, Parker MA, Jaroudi WA, Meine TJ, White JB, Elliott MD, Kim HW, Judd RM, Kim RJ. Detection of myocardial damage in patients with sarcoidosis. Circulation 2009;120:1969-77.

8. Cheong BY, Muthupillai R, Nemeth M, Lambert B, Dees D, Huber S, Castriotta R, Flamm S. The utility of delayed-enhancement magnetic resonance imaging for identifying non-ischemic myocardial fibrosis in asymptomatic patients with biopsy-proven systemic sarcoidosis. Sarcoidosis Vasc Diff Lung Dis 2009;26:3946.

9. Nadel J, Lancefield T, Voskoboinik A, Taylor AJ. Late gadolinium enhancement identified with cardiac magnetic resonance imaging in sarcoidosis patients is associated with long-term ventricular arrhythmia and sudden cardiac death. Eur Heart J Cardiovasc Imaging 2015;16:1634-41.

10. Crawford T, Mueller G, Sarsam S, Prasitdumrong H, Chaiyen N, Xioukui G, Schuller J, Kron J, Nour KA, Cheng A, Ji SY, Feinstein S, Gupta S, Ilg K, Sinno M, Abu-Hashish S, Al-Mallah M, Sauer WH, Ellenbogen K, Morady F, Bogun F . Magnetic resonance imaging for identifying patients with cardiac sarcoidosis and preserved or mildly reduced left ventricular function at risk of ventricular arrhythmias. Circ Arrhythm Electrophysiol 2014;7:1109-15.

11. Rudski LG, Lai WW, Afilalo J, Hua L, Handschumacher MD, Chandrasekaran K, Solomon SD, Louie EK, Schiller NB. Guidelines for the echocardiographic assessment of the right heart in adults: a report from the American Society of Echocardiography endorsed by the European Association of Echocardiography, a registered branch of the European Society of Cardiology, and the Canadian Society of Echocardiography. J Am Soc Echocardiogr 2010;23:685-13.

12. Gulati A, Ismail TF, Jabbour A, Alpendurada F, Guha K, Ismail NA, Jabbour A, Alpendurada F, Guha K, Ismail NA, Raza S, Khwaja J, Brown TDH, Morarji K, E Liodakis, Roughton M, Wage R, Pakrashi PC, Sharma R, Carpenter JP, Cook SA, Cowie MR, Assomull RG, Pennell DJ, Prasad SK. The prevalence and prognostic significance of right ventricular systolic dysfunction in non-ischemic dilated cardiomyopathy. Circulation 2013;128(15):1623-36.

13. Epstein AE, DiMarco JP, Ellenbogen KA, Estes NA 3 ${ }^{\text {rd }}$, Freedman RA, Gettes LS, Gillinov AM, Gregoratos G, Hammill SC, Hayes DL, Hlatky MA, Newby LK, Page RL, Schoenfeld MH, Silka MJ, Stevenson LW, Sweeney MO, Smith SC Jr, Jacobs AK, Adams CD, Anderson JL, Buller CE, Creager MA, Ettinger SM, Faxon DP, Halperin JL, Hiratzka LF, Hunt SA, Krumholz HM, Kushner FG, Lytle BW, Nishimura RA, Ornato JP, Page RL, Riegel B, Tarkington LG, Yancy CW. American College of Cardiology/American Heart Association Task Force on Practice Guidelines (Writing Committee to Revise the ACC/AHA/NASPE 2002 Guideline Update for Implantation of Cardiac Pacemakers and Antiarrhythmia Devices); American Association for Thoracic Surgery; Society of Thoracic Surgeons. ACC/AHA/HRS 2008 guidelines for device-based therapy of cardiac rhythm abnormalities. Circulation 2008;117:e350-e408.

14. Birnie DH, Sauer WH, Bogun F, Cooper JM, Culver DA, Duvernoy CS, Judson MA, Kron J, Mehta D, Nielsen JC, Patel AR, Ohe T, Raatikainen P, Soejima K. HRS Expert consensus statement on the diagnosis and management of arrhythmias associated with cardiac sarcoidosis. Heart Rhythm 2014;11(7):1305-23. 
15. Halushka MK, Yuh DD, Russell SD. Right ventricle-dominant cardiac sarcoidosis with sparing of the left ventricle. J Heart Lung Transplant 2006;25:479-82.

16. Murtagh G, Laffin LJ, Beshai JF, Maffessanti F, Bonham CA, Patel AV, Ju Z, Addetia K, Mor-Avi V, Moss JD, Hogarth DK, Sweiss NJ, Lang RM, Patel AR. Prognosis in myocardial damage in sarcoidosis patients with preserved left ventricular ejection fraction. Risk stratification using cardiac magnetic resonance. Circ Cardiovasc Imag 2016;9:e003738.

17. Schuller JL, Zipse M, Crawford T, Bogun F, Beshai J, Patel AR, Sweiss NJ, Nguyen DT, Aleong RG, Varosy PD, Weinberger HD, Sauer WH. Implantable cardioverter defibrillator therapy in patients with cardiac sarcoidosis. J Cardiovasc Electrophysiol 2012;23:925-9.

18. Patel AR, Klein MR, Chandra S, Spencer KT, DeCara JM, Lang RM, Burke MC, Garrity ER, Hogarth DK, Archer SL, Sweiss NJ, Beshai AF. Myocardial damage in patients with sarcoidosis and preserved left ventricular systolic function: an observational study. Eur J Heart Fail 2011;13:1231-37.

19. Muser D, Santangeli P, Patahk RK, Castro SA, Liang JJ, Magnani S, Hayashi T, Garcia FC, Hutchinson MD, Supple GE, Frankel DS, Riley RP, Lin D, Schaller RD, Desjardin B, Dixit S, Callans DJ, Zado ES, Marchlinski FE. Long-term outcomes of catheter ablation of ventricular tachycardia in patients with cardiac sarcoidosis. Circ Arrhythm Electr 2016;9(8):e004333.

20. Virmani R, Butres JC, Roberts WC. Cardiac sarcoidosis. A major cause of sudden death. Chest 1980;77:42328.

21. Roberts WC, Chung MS, Ko JM, Capehart JE, Hall SA. Morphologic features of cardiac sarcoidosis in native heart of patients having cardiac transplantation. Am J Cardiol 2014;113:706-12.

22. Roberts WC, McAllister HA Jr, Ferrans VJ. Sarcoidosis of the heart. A clinico-pathologic study of 35 necropsy patients (group I) and review of 78 previously described necropsy patients (group II). Am J Med 1977;63:86-108.

23. Tavora F, Creswell N, Li L, Ripple M, Solomon C, Burke A. Comparison of necropsy findings in patients with sarcoidosis dying suddenly from cardiac sarcoidosis versus dying suddenly from other causes. Am J Cardiol 2009;104:571-77.

24. Bagwan IN, Hooper LVB, Shepard MN. Cardiac sarcoidosis and sudden death. The heart may look normal or mimic other cardiomyopathies. Virchow Arch 2011;458:671-78.

25. Ekström K, Lehtonen J, Hänninen H, Kandolin R, Kivistö S, Kupari M. Magnetic resonance imaging as a predictor of survival free of life-threatening arrhythmias and transplantation in cardiac sarcoidosis. J Am Heart Assoc 2016;5:e003040.

26. Swift AJ, Rajaram S, Capener D, Elliott C, Condliffe R, Wild JM, Kiely DJ. LGE Patterns in pulmonary hypertension do not impact overall mortality. J Am Coll Cardiol Img 2014;7:1209-17.

27. Baughman RP, Engel PJ, Nathan S. Pulmonary hypertension in sarcoidosis. Clin Chest Med 2015;36:703-14.

28. Nunes H, Humbert M, Capron F, Brauner M, Sitbon O, Battesti JP, Simonneau G, Valeyre D. Pulmonary hypertension associated with sarcoidosis: Mechanisms, hemodynamics and prognosis. Thorax 2006;61:68-74.

29. Blankstein R, Osborn M, Naya M, Waller A, Kim CK, Murthy VL, Kazemian P, Kwong RY, Tokuda M, Skali H, Padera R, Hainer J, Stevenson WG, Dorbala S, Di Carli MF . Positron emission tomography enhances prognostic assessments of patients with suspected cardiac sarcoidosis. J Am Coll Cardiol 2014:63:329-36.

30. Philips B, Madhavan S, James CA, te Riele A, Murray B, Tichnell C, Bhonsale A, Nazarian S, Judge DP, Calkins H, Tandri H, Cheng A. Arrhythmogenic Right Ventricular Dysplasia/Cardiomyopathy and Cardiac Sarcoidosis. Distinguishing features when the diagnosis is unclear. Circ Arrhythm Electrophysiol 2014;7:23036.

31. Kuribayashi T, Roberts WC. Myocardial disarray at junction of ventricular septum and left and right ventricular free walls in hypertrophic cardiomyopathy. Am J Cardiol 1992;70:1333-40.

32. Sato T, Tsujino I, Ohira H, Oyama-Manabe N, Ito YM, Noguchi T, . Paradoxal interventricular septal motion as a major determinant of late gadolinium enhancement in ventricular insertion points in pulmonary hypertension. PLOs one 2013;8(6):e66724.

33. Gomez A, Bialostozky D, Zajarias A, Santos E, Palomar A, Martinez ML, Sandoval J. Right ventricular ischemia in patients with primary pulmonary hypertension. J Am Coll Cardiol 2001;38(4):1137-41.

34. McCann GP, Beek AM, Vonk-Noordegraaf A, van Rossum AC. Delayed contrast-enhanced magnetic resonance imaging in pulmonary arterial hypertension. Circulation 2005;112:e268.

35. Zorzi A, Perazzolo Marra M, Rigato I, De Lazzari M, Susana A, Niero A, Pilichou K, Migliore F, Rizzo S, Giorgi B, De Conti G, Sarto P, Serratosa L, Patrizi G, De Maria E, Pelliccia A, Basso C, Schiavon M, Bauce 


\section{Chapter 7}

B, Iliceto S, Thiene G, Corrado D. Non-Ischemic left ventricular scar as a substrate of life-threatening ventricular arrhythmias and sudden cardiac death in competitive athletes. Circ Arrhythm Electrophysiol 2016:9:e004229.

36. Ise T, Hasegawa T, Morita Y, Yamada M, Funada A, Takahama H, Amaki M, Kanzaki H, Okamura H, Kamakura S, Shimizu W, Anzai T, Kitakaze. Extensive late gadolinium enhancement on cardiovascular magnetic resonance predicts adverse outcomes and lack of improvement in LV function after steroid therapy in cardiac sarcoidosis. Heart 2014;100:1165-72.

37. Crouser ED, Ono C, Tran T, He X, Raman SV. Improved detection of cardiac sarcoidosis using magnetic resonance with myocardial T2 mapping. Am J Resp Crit Care Med 2014;189:109-112 
Table 1: Characteristics of patients with and without RV Late Gadolinium Enhancement

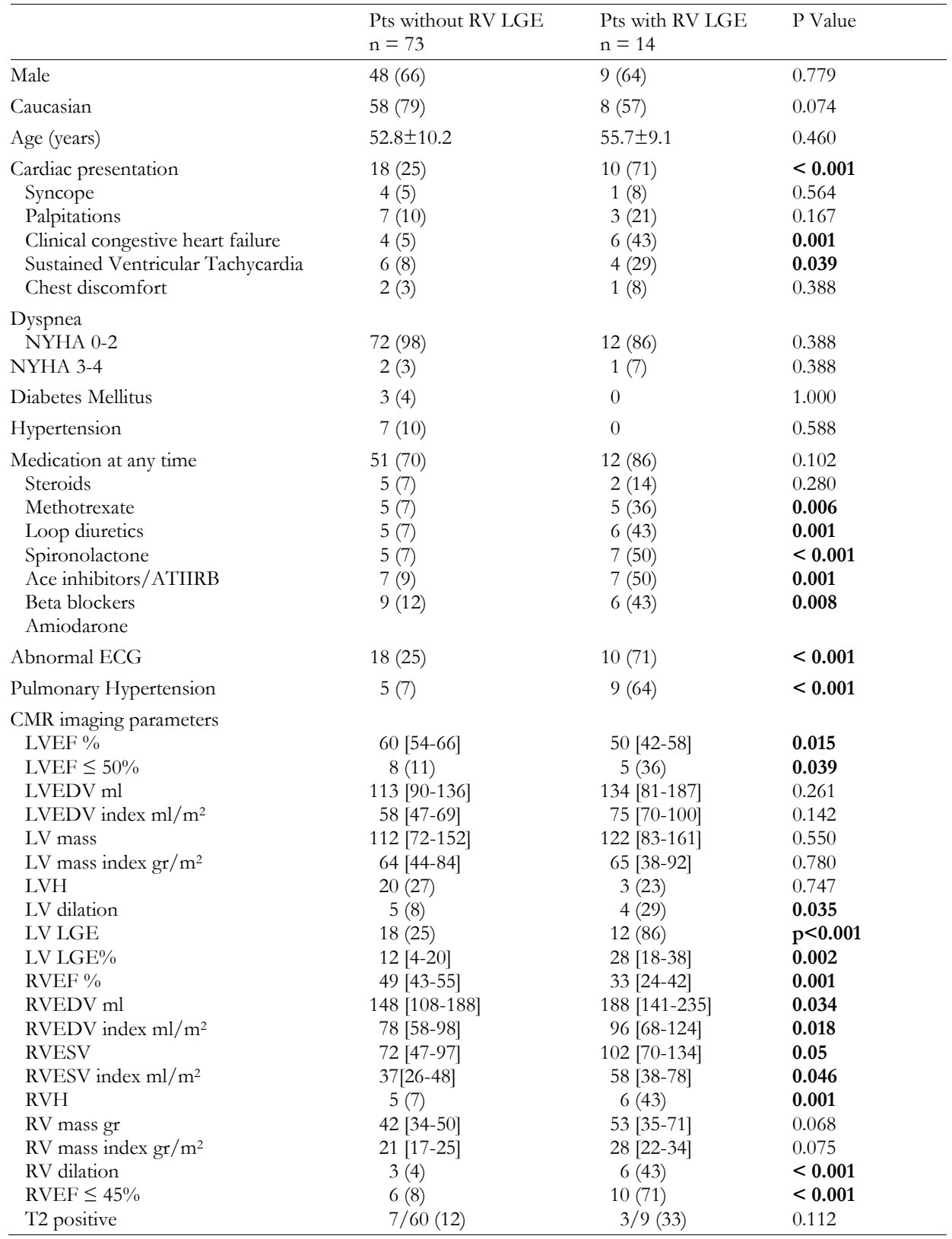

Values are $\mathrm{n}(\%)$, median $[\mathrm{IQR}]$, or mean $\pm \mathrm{SD} . *$ Values are for all patients with sarcoidosis $(87) . \mathrm{CI}=$ confidence interval; $\mathrm{CMR}=$ cardiac magnetic resonance; $\mathrm{EDV}=$ end-diastolic volume; $\mathrm{LGE}=$ late gadolinium enhancement; $\mathrm{LV}=$ left ventricle; $\mathrm{LVEDV}=$ left ventricular end-diastolic volume; $\mathrm{LVEF}=$ left ventricular ejection fraction; $\mathrm{RV}$ = right ventricle; RVEDV = right ventricular end-diastolic volume; RVEDVI = right ventricular end-diastolic volume index; RVEF = right ventricular ejection fraction; RVH = right ventricular hypertrophy. 


\section{Chapter 7}

Table 2: Characteristics of patients with RV Late Gadolinium Enhancement

\begin{tabular}{|c|c|c|c|c|c|}
\hline $\begin{array}{l}\text { Enhanced } \\
\text { segments }\end{array}$ & $\begin{array}{l}\text { Patients } \\
(\mathrm{n}=14)\end{array}$ & $\begin{array}{l}\text { Combination of enhanced } \\
\text { segments }\end{array}$ & $\begin{array}{l}\text { Patients } \\
(\mathrm{n}=14)\end{array}$ & $\begin{array}{l}\text { Patients with } \\
\text { pulmonary } \\
\text { hypertension }\end{array}$ & $\begin{array}{l}\text { Patients with end- } \\
\text { systolic septal shift } \\
(\mathrm{n}=5)\end{array}$ \\
\hline RV septal & 11 & $\begin{array}{l}\text { RV septal } \\
\text { VIP } \\
\text { RV free wall } \\
\text { RVOT }\end{array}$ & 1 & 1 & 0 \\
\hline VIP & 10 & $\begin{array}{l}\text { RV septal } \\
\text { VIP } \\
\text { RV free wall }\end{array}$ & 3 & 2 & 1 \\
\hline RV free wall & 8 & $\begin{array}{l}\text { RV septal } \\
\text { VIP }\end{array}$ & 4 & 1 & 0 \\
\hline \multirow[t]{5}{*}{ RVOT } & 1 & $\begin{array}{l}\text { RV septal } \\
\text { RV free wall }\end{array}$ & 2 & 2 & 1 \\
\hline & & RV septal & 1 & 1 & 1 \\
\hline & & RV free wall & 1 & 0 & \\
\hline & & VIP & 1 & 1 & 1 \\
\hline & & $\begin{array}{l}\text { RV free wall } \\
\text { VIP }\end{array}$ & 1 & 0 & 0 \\
\hline
\end{tabular}

RV - right ventricular; RVOT - right ventricular outflow tract, VIP - ventricular insertion points 
Table 3: CMR studies reporting on right ventricular involvement in cardiac sarcoidosis.

\begin{tabular}{|c|c|c|c|}
\hline Authors & Type of study & Patients & Conclusion \\
\hline $\begin{array}{l}\text { Cheong BYC et al. } \\
(2009) \\
(8)\end{array}$ & $\begin{array}{l}\text { prospective, } \\
\text { single-centre }\end{array}$ & $\begin{array}{l}31 \text { pts asymptomatic biopsy proven } \\
\text { systemic sarcoidosis, } 8(26 \%) \text { LV LGE } \\
\text { of whom } 2(25 \%) \text { with RV LGE, } \\
\text { inferobasal RV LGE in pts with most } \\
\text { LV LGE }\end{array}$ & $\begin{array}{l}\text { asymptomatic small amount of } \\
\text { LGE (average } 3.2 \% \text { of LV) in } 26 \% \text {, } \\
\text { no cardiac events after } 1 \mathrm{yr}\end{array}$ \\
\hline $\begin{array}{l}\text { Patel MR, et al. } \\
(2009) \\
(7)\end{array}$ & $\begin{array}{l}\text { prospective, } \\
\text { single-centre }\end{array}$ & $\begin{array}{l}81 \text { pts with extra-cardiac sarcoidosis, } \\
21(26 \%) \text { with LV LGE (average } 6 \\
\text { grams), } 14(67 \%) \text { had right-sided septal } \\
\text { LGE incl } 4 \text { RV free wall/outflow } \\
\text { tract/anterobasal segments }\end{array}$ & $\begin{array}{l}\text { pts with LGE had } 9 \text { fold higher rate } \\
\text { of adverse events } \\
\text { l }\end{array}$ \\
\hline $\begin{array}{l}\text { Patel AR, et al. } \\
\text { (2011) } \\
(18)\end{array}$ & $\begin{array}{l}\text { retrospective, } \\
\text { single-centre }\end{array}$ & $\begin{array}{l}152 \text { pts extra-cardiac sarcoidosis, } \\
\text { LVEF } \geq 50 \%, 29(19 \%) \text { LV LGE, no } \\
\text { data on RV LGE }\end{array}$ & $\begin{array}{l}\text { pts with LV LGE had lower RVEF, } \\
\text { either because of presumed } \\
\text { biventricular disease and/or } \\
\text { pulmonary hypertension }\end{array}$ \\
\hline $\begin{array}{l}\text { Schuller JL, et al. } \\
(2012) \\
(17)\end{array}$ & $\begin{array}{l}\text { retrospective, } \\
\text { multi-centre }\end{array}$ & $\begin{array}{l}112 \text { CS pts with ICD's for primary or } \\
\text { secondary prevention, no data on } \\
\text { LGE }\end{array}$ & $\begin{array}{l}\text { impaired systolic LV and RV } \\
\text { function correlates with more ICD } \\
\text { therapy }\end{array}$ \\
\hline $\begin{array}{l}\text { Samar HY et al. } \\
\text { (2014) (poster) } \\
\text { (3) }\end{array}$ & $\begin{array}{l}\text { retrospective, } \\
\text { single-centre }\end{array}$ & $\begin{array}{l}122 \text { sarcoidosis pts, } 37(22 \%) \text { LV } \\
\text { LGE, } 18(49 \%) \text { of these also RV LGE }\end{array}$ & $\begin{array}{l}\text { LVEF, LVEDV, RVEDV similar in } \\
\text { groups with/without RV LGE }\end{array}$ \\
\hline $\begin{array}{l}\text { Crawford T, et al. } \\
\text { (2014) } \\
(10)\end{array}$ & $\begin{array}{l}\text { retrospective, } \\
\text { multi-centre }\end{array}$ & $\begin{array}{l}52 \text { CS pts, all LVEF }>35 \% 32(62 \%) \\
\text { with LV LGE of which } 13(41 \%) \text { also } \\
\text { had RV LGE }\end{array}$ & $\begin{array}{l}\text { multi-focal LGE correlated with } \\
\text { VT/VF, pts with RV LGE had } \\
\text { more extensive LV LGE }\end{array}$ \\
\hline $\begin{array}{l}\text { Nadel J, et al. } \\
(2015) \\
(9)\end{array}$ & $\begin{array}{l}\text { retrospective, } \\
\text { single-centre }\end{array}$ & $\begin{array}{l}106 \text { sarcoidosis pts, } 32 \text { CS-defined by } \\
\text { CMR LGE - } 32 \text { LV LGE, } 2 \text { (6\%) RV } \\
\text { LGE }\end{array}$ & $\begin{array}{l}\text { LGE only independent predictor of } \\
\text { adverse outcome }\end{array}$ \\
\hline $\begin{array}{l}\text { Muser D, et al. } \\
(2015) \\
(19)\end{array}$ & $\begin{array}{l}\text { prospective, } \\
\text { single-centre }\end{array}$ & $\begin{array}{l}31 \text { CS pts with VTs pre-ablation, } 23 \\
\text { had CMR, 21(68\%) LV LGE, } 11 \\
(35 \%) \text { RV LGE, no data on RV } \\
\text { distribution or extent }\end{array}$ & $\begin{array}{l}\text { LGE extent predicted VT-free } \\
\text { survival }\end{array}$ \\
\hline $\begin{array}{l}\text { Ekström K, et al. } \\
(2016) \\
(25)\end{array}$ & $\begin{array}{l}\text { retrospective, } \\
\text { single-centre }\end{array}$ & $\begin{array}{l}50 \text { CS pts, } 48(96 \%) \text { with LV LGE, } \\
\text { not reported on RV LGE }\end{array}$ & $\begin{array}{l}\text { LV extent of LGE and RVEF, } \\
\text { correlated with adverse outcome }\end{array}$ \\
\hline $\begin{array}{l}\text { Murtagh G, et al. } \\
(2016) \\
(16)\end{array}$ & $\begin{array}{l}\text { retrospective, } \\
\text { single-centre }\end{array}$ & $\begin{array}{l}205 \text { pts extra-cardiac sarcoidosis, } \\
\text { LVEF } \geq 50 \%, 41(20 \%) \text { LV LGE, } \\
\geq 4 \text { pts with VIP LGE, no specific } \\
\text { data on RV LGE }\end{array}$ & $\begin{array}{l}\text { for every } 1 \% \text { in LGE burden the } \\
\text { hazard for an event increased by } \\
8 \% \text {; mild impaired RV dysfunction } \\
\text { correlated with increased event rate }\end{array}$ \\
\hline
\end{tabular}

CS - cardiac sarcoidosis, LGE - late gadolinium enhancement, LV - left ventricle, LVEF - left ventricular ejection fraction, RV - right ventricle, RVEF - right ventricular ejection fraction 


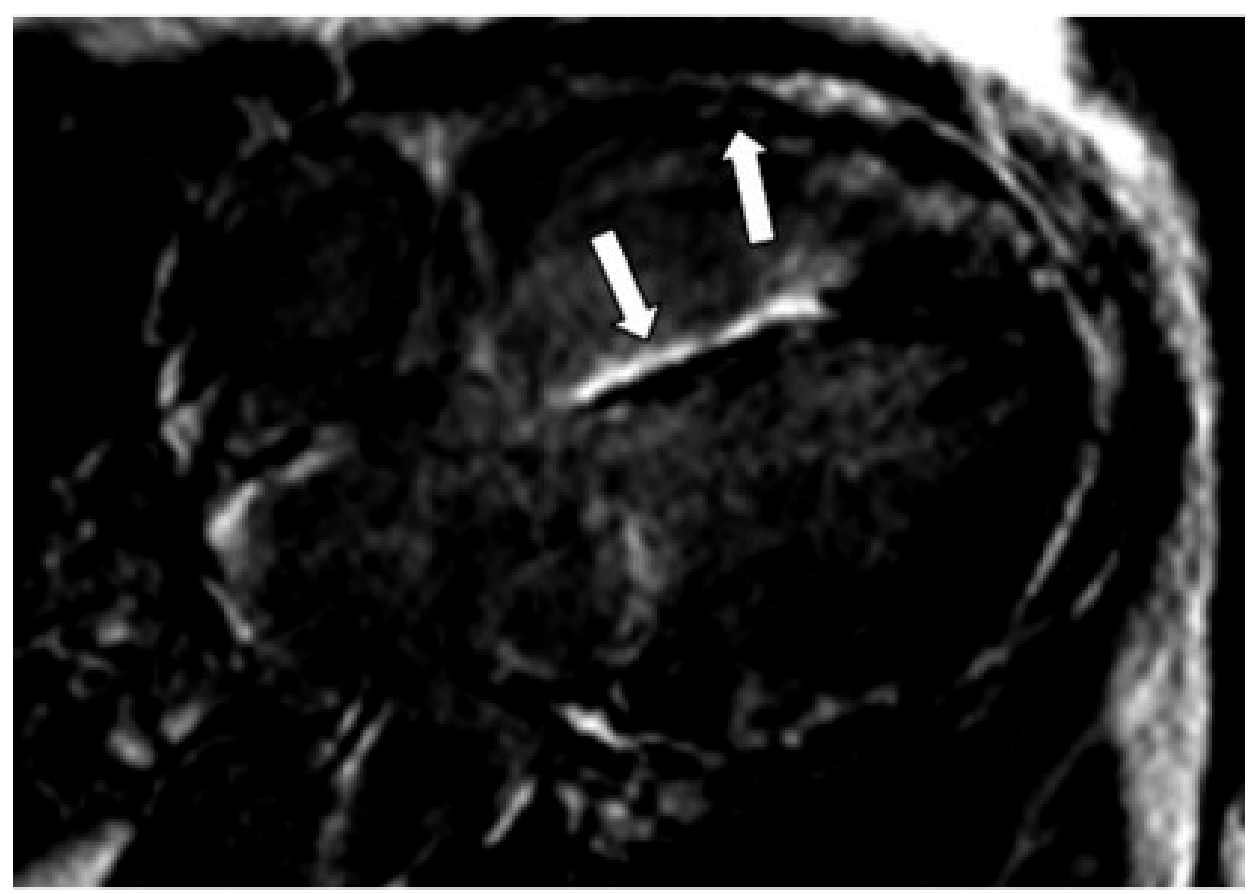

Figure 1A: Contrast-enhanced magnetic resonance study in a patient with biventricular congestive heart failure (Inversion-Recovery Gradient Echo sequence, horizontal long axis view) demonstrates enhancement of the right ventricular free wall and right-sided interventricular septum. 
Right ventricular involvement in cardiac sarcoidosis

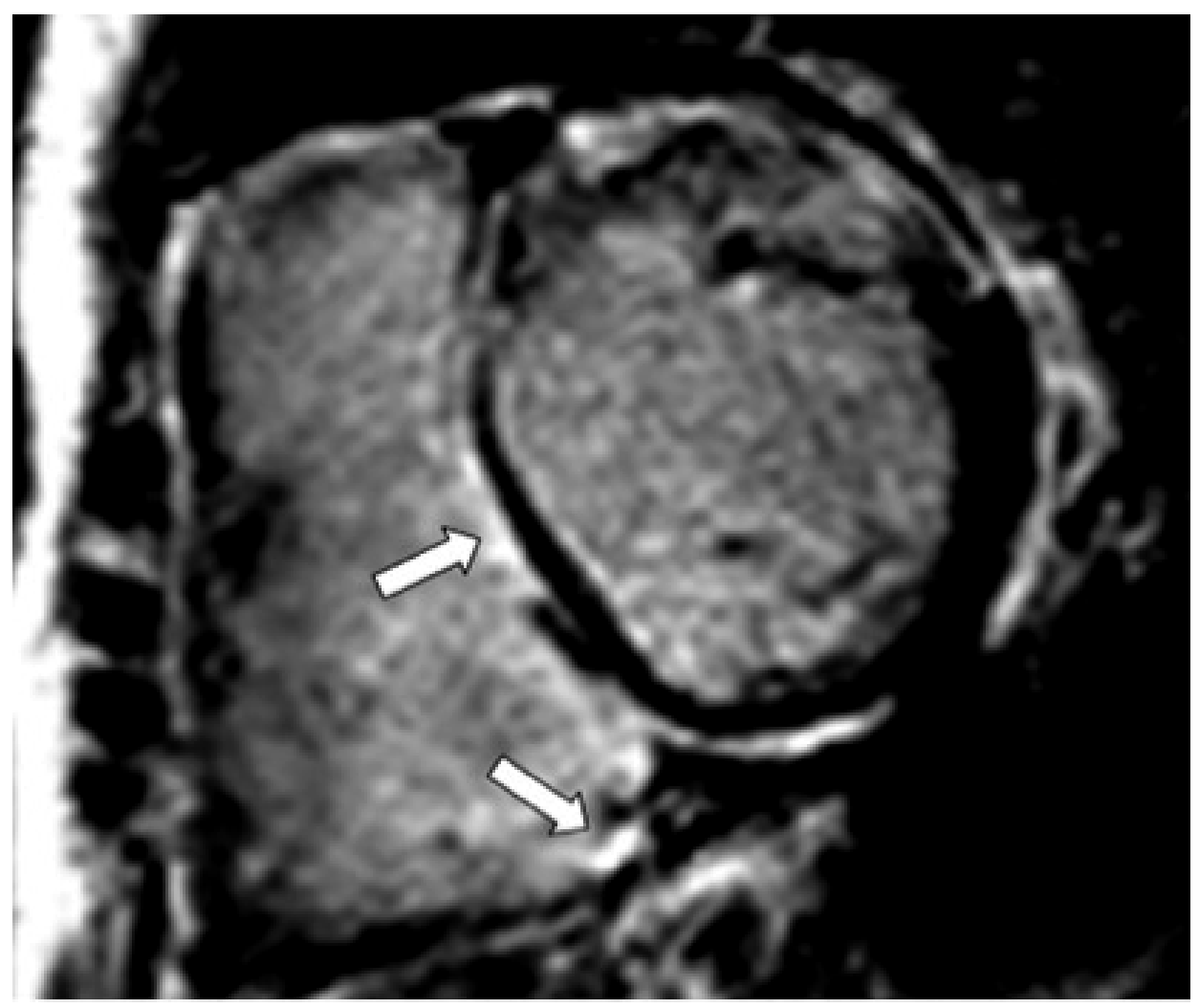

Figure 1B: Short axis view in the same patient demonstrates enhancement of the right-sided interventricular septum and inferior right ventricular insertion point. 


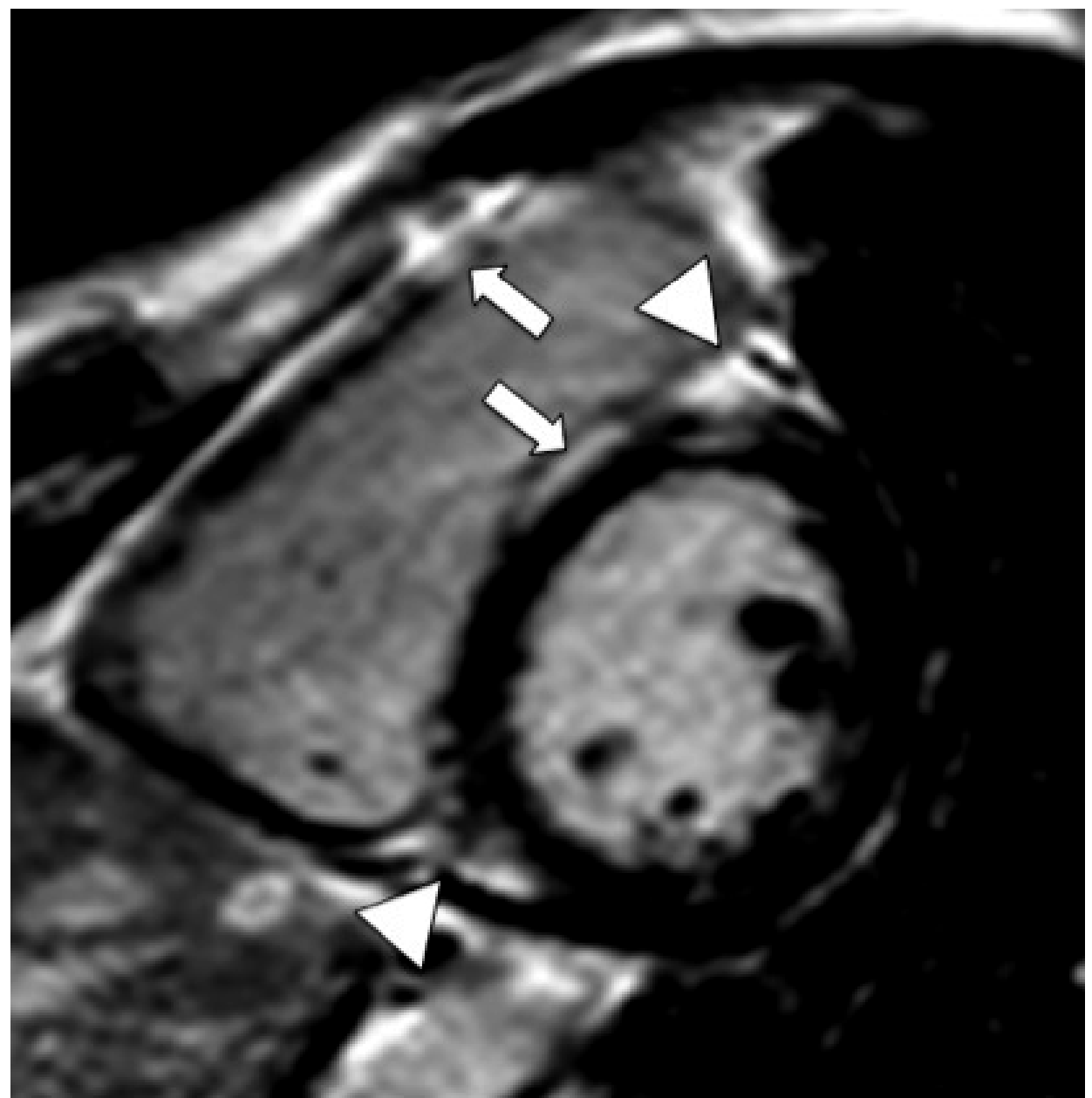

Figure 2: Contrast-enhanced magnetic resonance study (Inversion-Recovery Gradient Echo sequence, enddiastolic frame, short axis view) demonstrates enhancement of the right ventricular free wall (arrow), ventricular insertion points (triangles) and right-sided septum (arrow). 


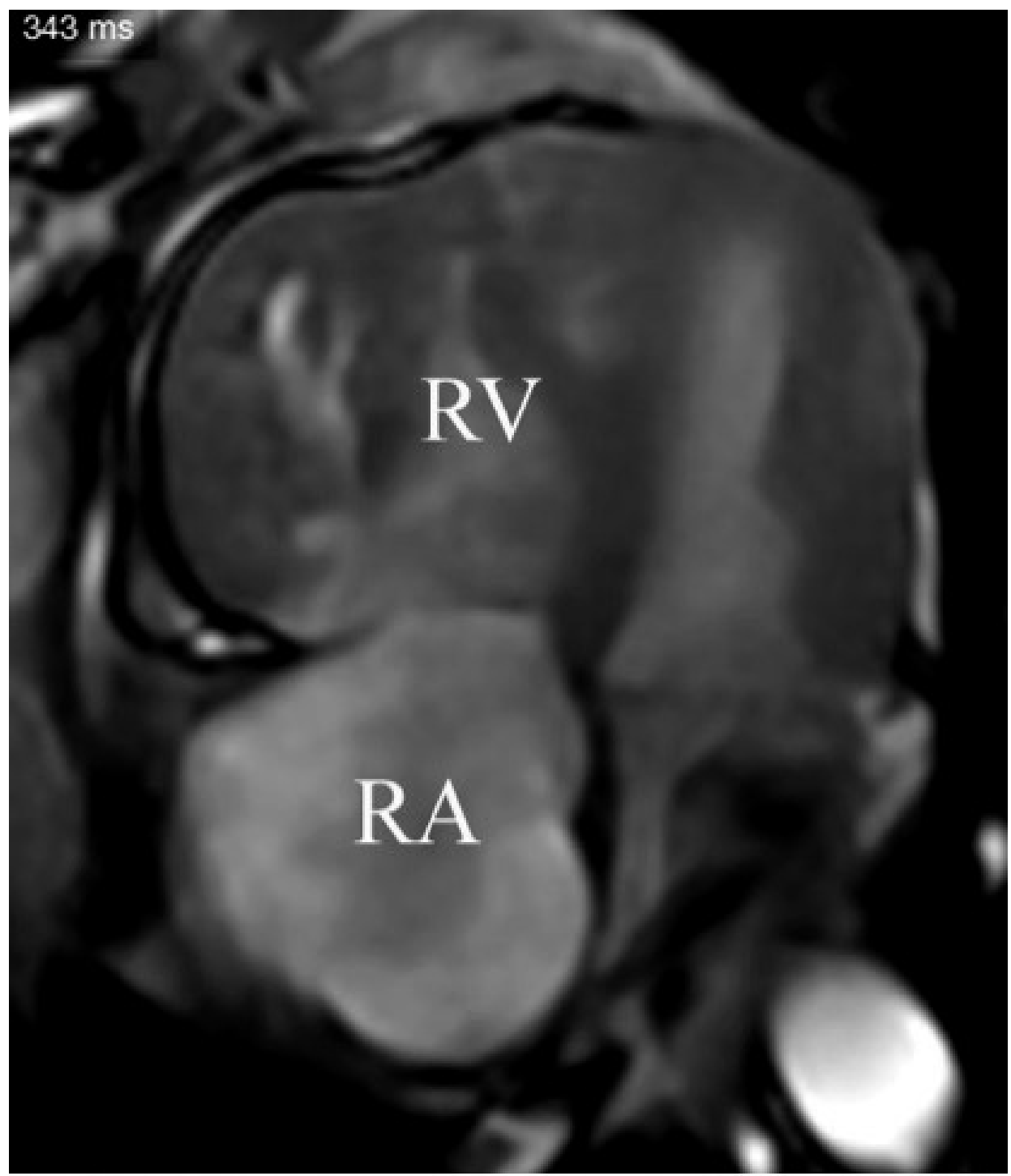

Figure 3A: Magnetic resonance study (Steady-State-Free Precession sequence, horizontal long axis view, enddiastolic frame), demonstrates dilation of the right ventricle (RV) and right atrium (RA), marked right ventricular hypertrophy, with displacement of the interventricular septum towards the left ventricle, both left ventricle and atrium are compressed. 


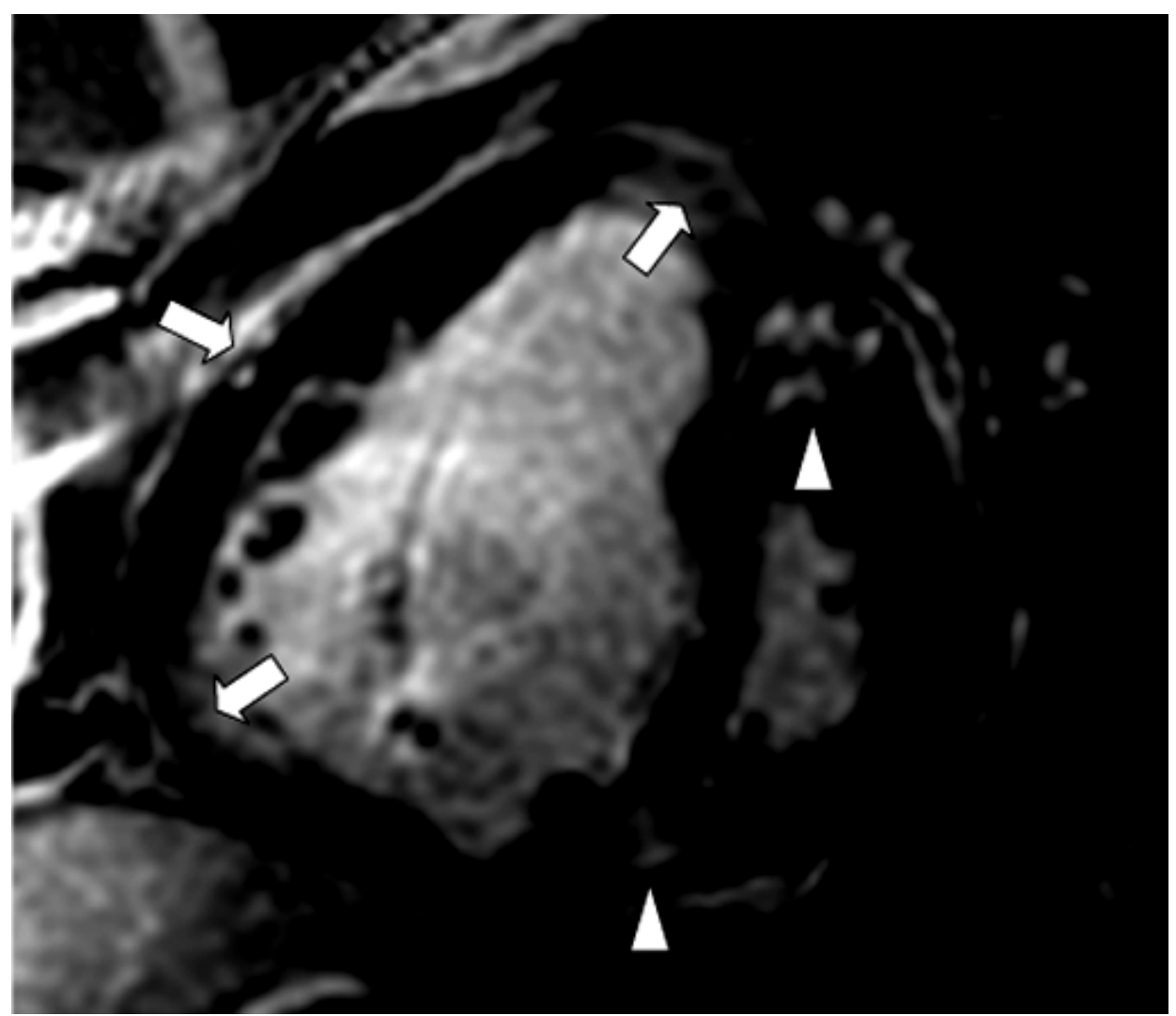

Figure 3B: Contrast-enhanced magnetic resonance study (Inversion Recovery-Gradient Echo sequence, short axis view, end-diastolic frame) of the identical patient with pulmonary vascular sarcoidosis and resulting severe pulmonary arterial hypertension, demonstrates contrast-enhancement of the right ventricular hinge points (triangles) and free wall (arrows). 


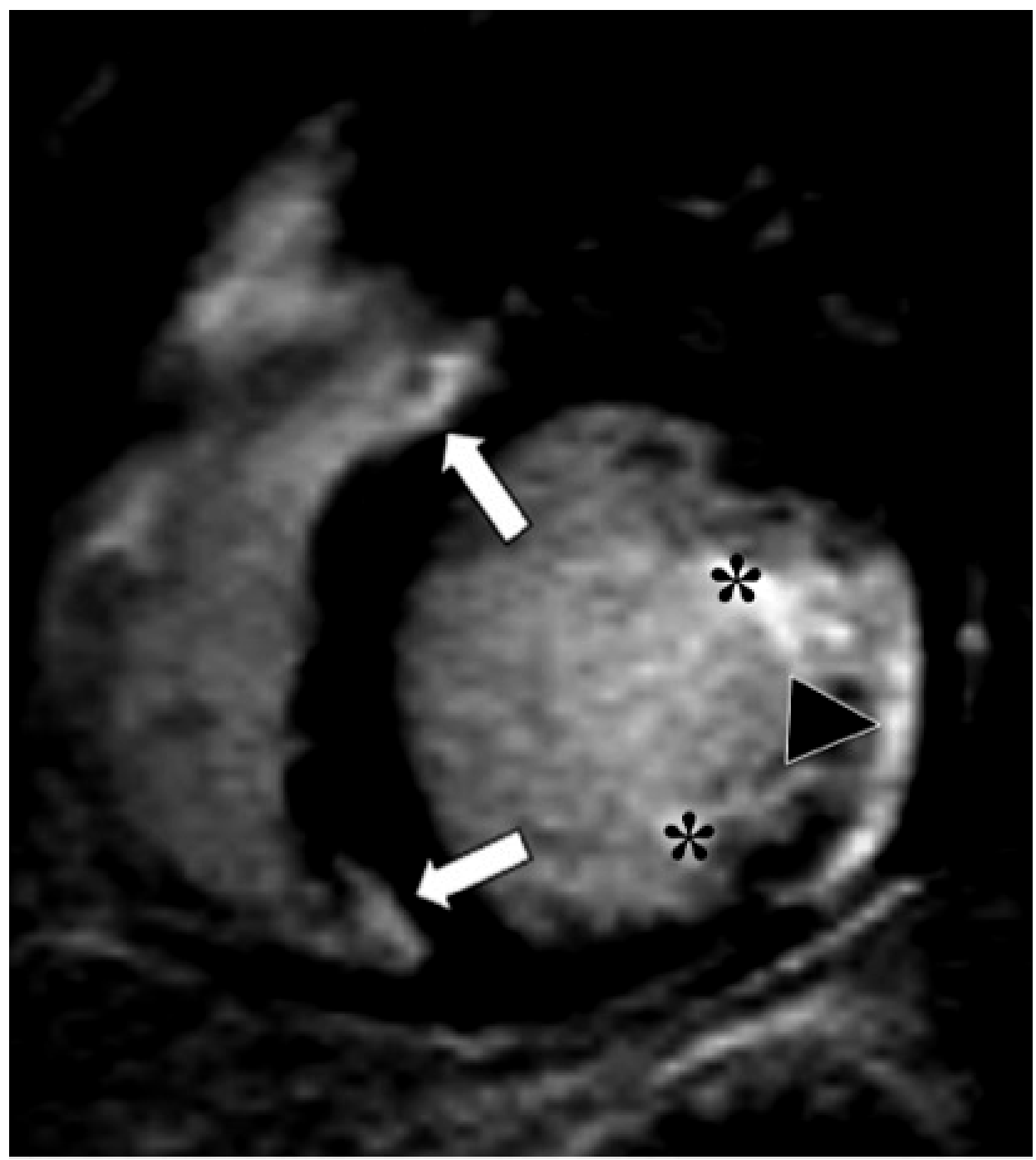

Figure 4: Contrast-enhanced magnetic resonance study (Inversion-Recovery Gradient Echo sequence, short axis view) in a patient without pulmonary hypertension demonstrates enhancement of the ventricular insertion points (arrows), papillary muscles (asterisks) and postero-lateral left ventricular segments (triangle). 


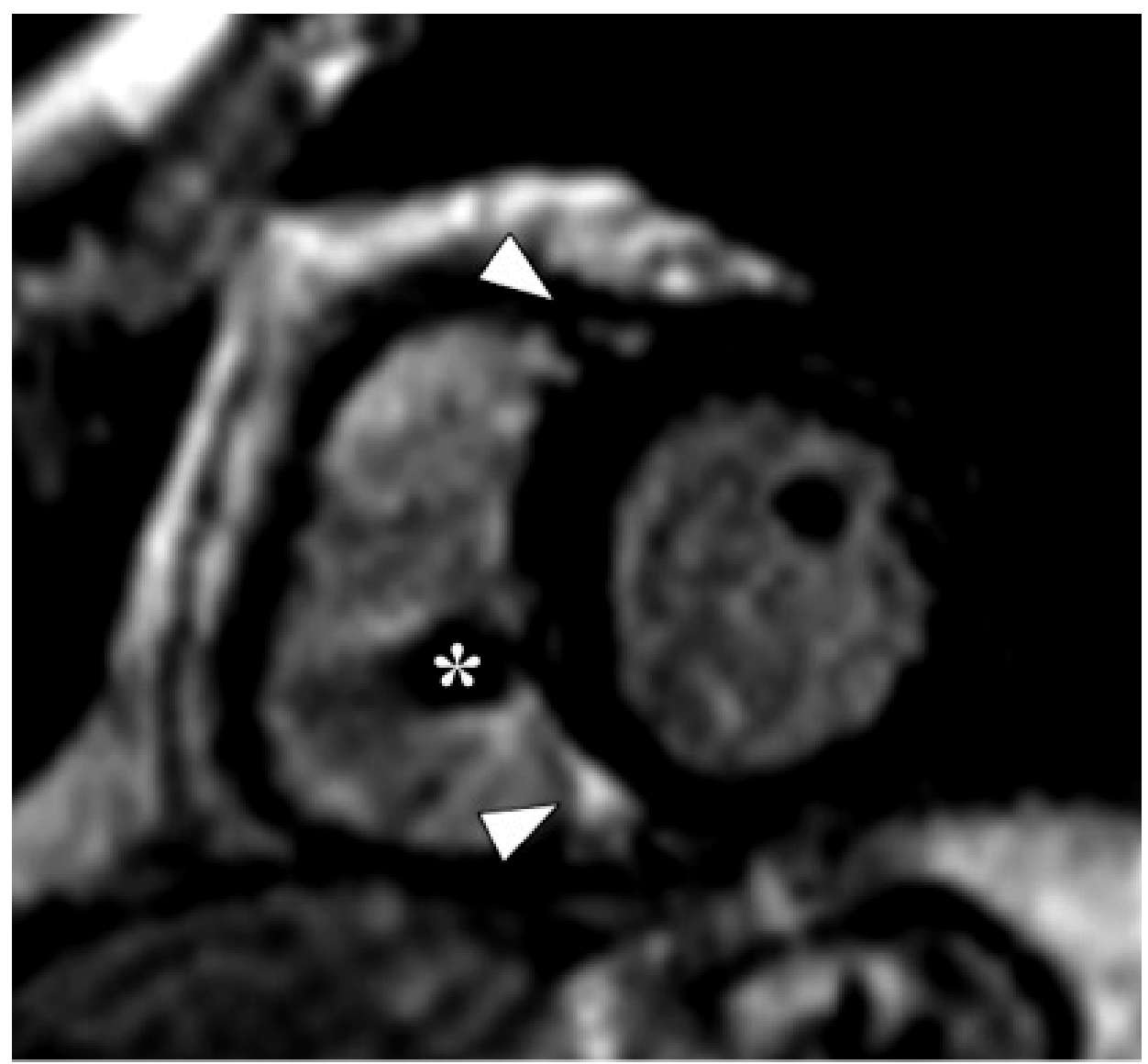

Figure 5A: Contrast-enhanced magnetic resonance study (Inversion-Recovery Gradient Echo sequence, enddiastolic frame, short axis view) in a patient diagnosed with a high degree atrio-ventricular block secondary to active cardiac sarcoidosis. A dual chamber pacemaker had been inserted. Ventricular insertion point enhancement is demonstrated (triangles). Pulmonary pressures were normal. (asterisk - artifact of right ventricular pace lead) 


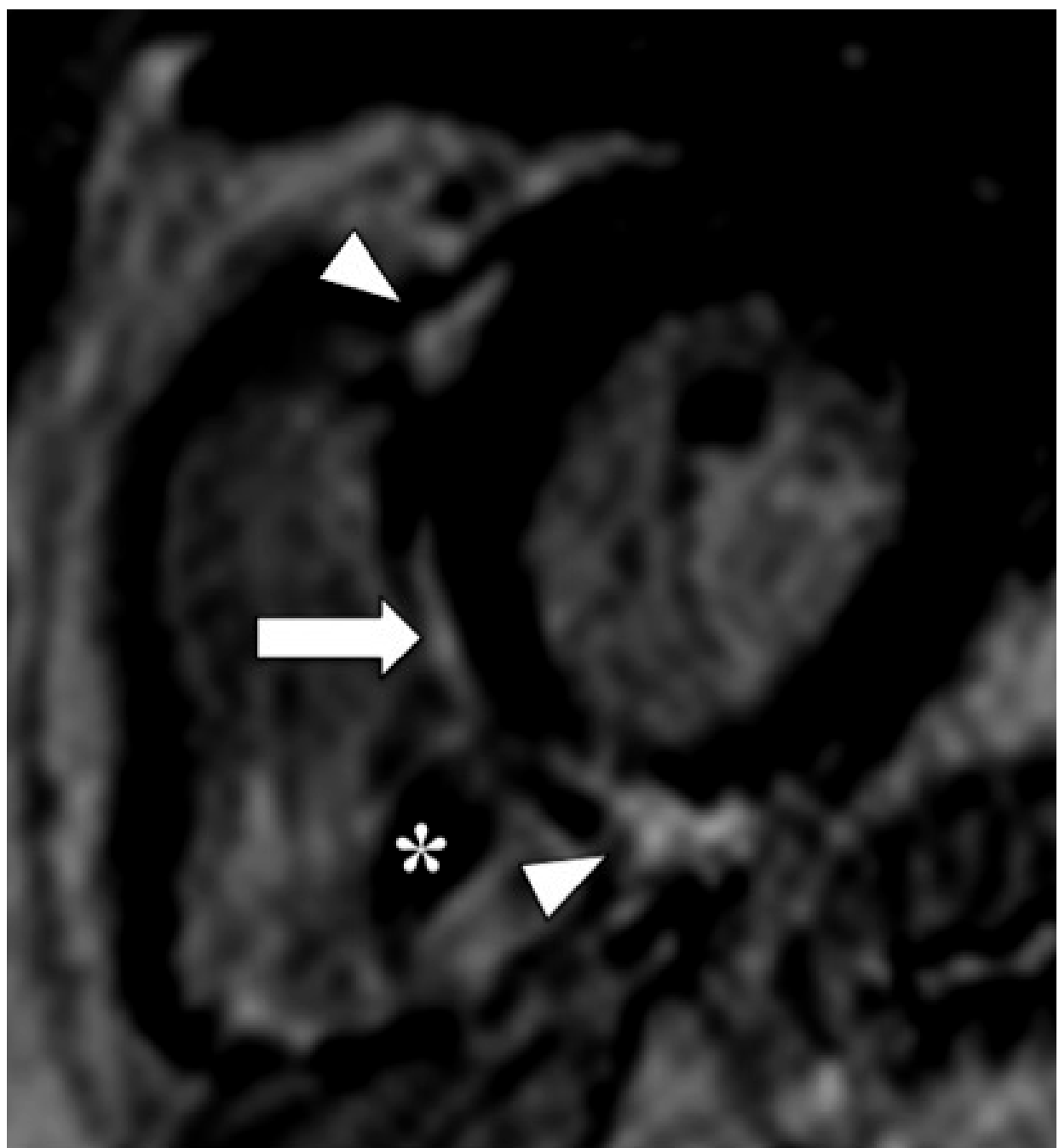

Figure 5B: Contrast-enhanced magnetic imaging study (Inversion-Recovery Gradient Echo sequence, enddiastolic frame, short axis view) in the identical patient when reassessed 7 years later demonstrates substantially more enhancement of the right-sided septum (arrow) and the insertion points (triangles). The percentage time pacing had increased from $5 \%$ to $15 \%$ of the time. (asterisk = artifact produced by right ventricular pace lead) 



\section{Chapter 8}

\section{Right ventricular involvement and the extent of left ventricular enhancement with magnetic resonance predict adverse outcome in pulmonary sarcoidosis}

ESC Heart Failure 2018;5(1):157-171.

Jan-Peter Smedema ${ }^{1}, \mathrm{MD}, \mathrm{MSc}$, Robert-Jan van Geuns ${ }^{2}, \mathrm{MD}, \mathrm{PhD}$, Joris Ector ${ }^{3}, \mathrm{MD}$, $\mathrm{PhD}$, Hein Heidbuchel ${ }^{4}$, MD, PhD, Gillian Ainslie ${ }^{5}$, MB ChB, FRCP, Harry J.G.M.Crijns ${ }^{1}$, $\mathrm{MD}, \mathrm{PhD}$.

${ }^{1}$ Department of Cardiology, Maastricht University Medical Centre, Maastricht, The Netherlands. Email: hjgmcrijns@mumc.nl

2Department of Cardiology, Erasmus Medical Centre, Rotterdam, The Netherlands. Email: r.vangeuns@erasmusmc.nl

${ }^{3}$ Department of Cardiology, University Hospitals Gasthuisberg, Leuven, Belgium. Email: jorisector@gmail.com

4.University of Hasselt Heart Centre, Virga Jesse Hospital, Hasselt, Belgium. Email: heinheid@gmail.com

${ }^{5}$ Respiratory Clinic, Department of Medicine, Groote Schuur Hospital, Cape Town, epublic of South Africa. Email: Gillian.Ainslie@uct.ac.za 


\begin{abstract}
Objectives: To determine whether delayed right ventricular (RV) gadolinium enhancement (LGE) with cardiovascular magnetic resonance (CMR) predicts adverse events in addition to left ventricular LGE during long-term follow up of pulmonary sarcoidosis patients.
\end{abstract}

Background: Cardiac involvement is the main determinant of poor outcomes in sarcoidosis. Right ventricular dysfunction and LV LGE were reported to be predictive of adverse outcome in non-ischemic cardiomyopathies.

Methods: 84 consecutive biopsy proven pulmonary sarcoidosis patients were followed for a median of 56 months [38-74] after baseline DECMR. The composite primary end-point consisted of admission for congestive heart failure, sustained VT, appropriate ICD therapy, pacemaker implantation for high degree atrio-ventricular block, or cardiac death. The composite secondary end-point included all cause mortality in addition to the primary endpoint.

Results: RV and LV LGE were demonstrated in respectively 12 and 27 patients. Five of ten events included in the primary end-point occurred in the group with RV LGE. RV LGE, LV or biventricular LGE yielded Cox hazard ratios of 8.71 (95\% CI 1.90-23.81), 9.22 (95\% CI 1.96-43.45), and 12.09 (95\% CI 3.43-42.68) for the composite primary end-point. In a multi-variate model the predictive value of biventricular LGE for the composite primary and secondary end-points was strongest. Kaplan Meier event-free survival curves were most significant for RV LGE and biventricular LGE (log rank with $\mathrm{p}<0.001$ ).

Conclusions: Biventricular LGE at presentation is the strongest, independent predictor of adverse outcome during long-term follow up. Asymptomatic myocardial scar $<8 \%$ of LV mass carried a favorable long-term outcome.

\title{
Contents
}

Abstract 186

$\begin{array}{lll}8.1 & \text { Introduction } & 187\end{array}$

$\begin{array}{lll}8.2 \text { Methods } & 187\end{array}$

$\begin{array}{lll}8.3 & \text { Results } & 189\end{array}$

$\begin{array}{lll}8.4 & \text { Discussion } & 191\end{array}$

8.5 Study limitations: 193

$\begin{array}{lll}8.6 & \text { Conclusions } & 193\end{array}$

$\begin{array}{lll}8.7 & \text { Perspectives } & 193\end{array}$

8.8 Acknowledgements 194

$\begin{array}{ll}\text { Disclosures } & 194\end{array}$

$\begin{array}{ll}\text { References } & 195\end{array}$ 


\subsection{Introduction}

Sarcoidosis is a rare inflammatory condition of unknown etiology, which results in granulomatous infiltration and focal myocardial scar in approximately a third of patients. Major cardiac morbidity or mortality have been reported in approximately five to ten percent of sarcoidosis patients. (1) Diagnosing cardiac sarcoidosis (CS) can be challenging and several non-invasive diagnostic imaging modalities have been used over the years. Recently delayed contrast-enhanced cardiac magnetic resonance (DECMR) and positron electron tomography (PET) showed most diagnostic and prognostic promise. (2-15) DECMR has become the gold standard test for detecting and quantifying focal myocardial scar, a predictor of high degree atrio-ventricular block (AVB), heart failure (CCF), ventricular tachycardia (VT), and sudden cardiac death (SCD) in CS. (2-8,10-15) (Table 1) Systolic right ventricular (RV) impairment and multi-focal RV late gadolinium enhancement (LGE) on CMR, and active RV granulomatous inflammation on PET have been associated with adverse outcomes in retrospective studies. $(\mathbf{2}, 7,11,12,21,22,24)$ We previously demonstrated the diagnostic accuracy of DECMR for CS, and reported on RV involvement in pulmonary sarcoidosis utilizing CMR. $(\mathbf{1 6 , 2 5 )}$ We currently report on the prognostic value of RV LGE in addition to left ventricular (LV) LGE in patients suffering from pulmonary sarcoidosis.

\subsection{Methods}

\section{Patient population}

Between July 2001 and August 2010 we prospectively followed 84 consecutive patients with histologically proven pulmonary sarcoidosis. These patients had been referred for cardiac evaluation, either because of cardiac symptoms (palpitations, congestive heart failure, (pre)syncope, chest discomfort) or for routine cardiac assessment. (Figure 1) Patients were excluded in case of standard contraindications to DECMR. Approval for our project was obtained from the local Institutional Review Board.

\section{CMR protocol}

CMR studies were performed using a commercial $1.5 \mathrm{~T}$ MRI scanner with a cardiacdedicated, phased-array coil. The CMR studies were ECG triggered by standard software. Studies consisted of multi-phase multi-slice steady-state-free precession (SSFP), and fatsaturated T2-weighted spin-echo (64 patients) breath-hold sequences of the short axis, vertical long axis and horizontal long axis views. The short-axis images covered the LV and RV from base to apex. The SSFP sequences (typical repetition time: $3.5 \mathrm{msec}$; echo-time $1.4 \mathrm{msec}$; flip-angle $55^{\circ}$, temporal resolution $50 \mathrm{msec}$, voxel size $1.6 \times 1.6 \times 10 \mathrm{~mm}$, no gap) were performed to assess regional wall-motion abnormalities, ventricular volumes, masses and ejection fractions (LVEF, RVEF). T2-weigthed studies were performed to assess for the presence of myocardial inflammation. Contrast-enhanced and T2-weighted images were obtained in diastole to minimize artifact due to cardiac motion. Ten minutes after the addi- 
tional administration of $0.1 \mathrm{mmol} / \mathrm{kg}$ gadolinium-diethylenetriaminepenta-acetic acid (Schering, Berlin, Germany), a two-dimensional segmented inversion recovery-gradient echo breath-hold sequence (short axis, vertical long axis, and horizontal long axis, voxel size $1.6 \times 1.6 \times 10 \mathrm{~mm}$, without gap), was used to assess for LGE. The inversion time (250 to $400 \mathrm{~ms}$ ) was determined on an individual basis to obtain optimal nulling of the unenhanced myocardial signal.

\section{CMR analysis}

CMR studies were analyzed off-line by two experienced blinded observers who independently evaluated the study findings using commercially available software (CAAS MRV 3.4, Pie Medical Imaging, Maastricht, The Netherlands). Endocardial and epicardial contours were manually delineated in end-diastolic and end-systolic short axis slices to determine LV and RV end-diastolic volumes (LVEDV, RVEDV), end-systolic volume (LVESV, RVESV), LVEF, RVEF and LV and RV end-diastolic masses, which were indexed to body surface area. The presence and distribution of LGE and increased T2 signal, was determined by consensus, and LV LGE localized according to the 17-segment model. LGE was considered present only if confirmed on both short-axis and matching long-axis myocardial locations. LGE was quantified by a semiautomatic detection method using the signal intensity threshold of $\geq 2$ SD above a remote reference region. The distribution of LGE was characterized as sub-endocardial, mid wall, sub-epicardial, patchy or confluent trans-mural. When more than one pattern was present, the distribution was characterized on the basis of the predominant pattern. There was excellent intra- and interobserver correlation for ventricular volumes, masses, and ejection fractions. Interobserver agreement when determining the presence/localization of LGE was good (kappa 0.85, $\mathrm{p}<0.001$ ). The intra-class correlation coefficient for LV LGE was 0.989 [0.981-0.993] $(\mathrm{p}=0.001)$.

\section{Clinical follow-up}

Scheduling of follow up visits was 4-6 monthly and left at the discretion of the managing clinician. Resting 12-lead surface ECG's, and 24-72 hour ambulatory ECGs were performed, and evaluated for intermittent intra-ventricular or atrio-ventricular conduction disease, and/or ventricular arrhythmias. Sustained VT (sVT) was defined as VT with a rate $>100$ beats/min, lasting for at least 30 seconds. Implanted Cardioverter Defibrillators (ICD) or pacemakers (PM) were interrogated every 4 months and assessed for ventricular arrhythmias. At regular intervals and at the end of our study, outcome data were collected from the family physician and managing specialist.

\section{$V$ ariables, definitions, adverse events, and composite end-points}

The composite primary end-point consisted of newly developed AVB resulting in PM implantation, admission for CCF, sVT, appropriate ICD therapy for sVT or ventricular fibrillation (VF), or cardiac death. Appropriate ICD therapy was defined as ATP or shock for fast VT (R-R $<320 \mathrm{~ms}$ ) or VF. The composite secondary end-point included all-cause 
death in addition to the primary end-point. Peak systolic RV pressures over $40 \mathrm{~mm} \mathrm{Hg}$ were considered to represent pulmonary hypertension (PH). RV end-diastolic wall thickness over $5 \mathrm{~mm}$ was considered evidence of RV hypertrophy (RVH). RV dysfunction was defined as a right ventricular ejection fraction (RVEF) $<45 \%$ by CMR. (17)

Statistical Analysis: All statistical analyses were performed using statistical software (Version 21.0, SPSS; Chicago, IL). Continuous normally distributed data were expressed as mean $\pm \mathrm{SD}$, and between-group comparisons were made using the parametric $t$ test for independent samples or Mann-Whitney test when appropriate. In non-normally distributed continuous data, the median and interquartile ranges were determined, and between-group comparisons were made with the Wilcoxon test. Categorical variables were assessed using the chi-square or Fischer Exact test when appropriate. Linear regression analysis was used to determine the relationship between LGE, LVEDV and LVEF. Univariate analyses of the risk for adverse outcome associated with selected variables were performed with the Cox proportional hazards model. A $\mathrm{p}$ value of $<0.05$ was considered statistically significant. The hazard ratio (HR) for the prediction of events was calculated for each of the outcomes using a multivariable Cox regression model, two tailed values of $\mathrm{p}<0.05$ were considered significant. Composite event curves were determined according to the KaplanMeier method, and comparisons of cumulative event rates were performed using the logrank test. Receiver-operating characteristic (ROC) curves were used to examine the performance characteristic of \%LGE mass. Area under the curve (AUC), and 95\% confidence of the ROC curve, was calculated to provide a measure of the accuracy of $\%$ LGE mass to predict combined adverse outcomes.

\subsection{Results}

\section{Patient characteristics}

Figure 1 and Table 2 demonstrate the baseline characteristics of the included 84 patients. Twenty-nine patients had CS according to the HRS criteria, 18 according to the JMHW criteria. (19) Twenty-five $(30 \%)$ patients presented with cardiac symptoms (palpitations, (pre)syncope, chest discomfort, congestive heart failure) whilst the remaining 59 patients $(70 \%)$ experienced of non-specific symptoms (fatigue) or were routinely screened for CS. According to the ACC/AHA/HRS 2008 guidelines for device-based therapy of cardiac rhythm abnormalities, an ICD or PM were implanted in respectively 14 (4 ICD/biventricular PM) and 2 patients after the baseline DECMR study. (18) Heart failure was managed according to current optimal practice.

\section{CMR findings}

The findings with DECMR are displayed in Table 3. Twelve patients (44\%) had RV LGE, predominantly involving the right-sided interventricular septum, and/or ventricular insertion points. Two patients had isolated RV LGE. Patients with RV LGE had significantly 
more \%LV LGE than those without RV involvement (32\% [23-41] versus 10\% [2-18], $\mathrm{p}=0.006)$, and RV LGE correlated with the presence of PH ( $\mathrm{p}=0.022), \mathrm{RVH}(\mathrm{p}=0.050)$ and impaired RV function ( $\mathrm{p}<0.001)$.

LGE involved 4-39\% of LV mass, and predominantly involved the interventricular septum, and basal LV segments. LGE was equally distributed over the three myocardial layers, and was patchy (56\%) or confluent trans-mural (44\%). In 16 patients (59\%) three myocardial layers were involved. In twelve percent of patients $(7 / 59)$ who were routinely evaluated, asymptomatic myocardial scar was present, though significantly smaller when compared to the symptomatic group ( $8 \%$ [4-20] versus $28 \%$ [8-39], $\mathrm{p}=0.001)$. LGE strongly correlated with the presence of cardiac symptoms, ECG abnormalities, VT, LVEDV and LVEF $(p<0.001)$. The trans-mural extent of LGE correlated with segmental wall motion abnormalities $(\mathrm{p}<0.001)$. Linear regression analysis demonstrated a decrease in LVEF of $7.27 \%$ for every $10 \%$ increase in LGE $\left(\mathrm{r}^{2} 0.392\right.$, value $\left.\mathrm{p}<0.001,95 \% \mathrm{CI},-5.25 \%--9.30 \%\right)$. For every $10 \%$ increase in LGE the LVEDV increased $9.1 \mathrm{ml}$ in volume $\left(\mathrm{r}^{2} 0.239, \mathrm{p}<0.001\right.$, $95 \%$ CI $5.27 \mathrm{ml}-12.92 \mathrm{ml}$ ). In patients with sVT at presentation the median LVEF was significantly poorer, and LV mass, LVEDV index, and \%LV LGE significantly higher when compared to those without sVT at presentation (respectively 60\% [53-67] versus 51\% [34-68] ( $\mathrm{p}=0.001), 55 \mathrm{ml}$ [44-66], versus 73ml [50-95] ( $\mathrm{p}=0.011), 111$ [80-142] versus 145 gram [95-195] ( $p=0.028)$, and 14 (range $2-38)$ versus 33\% [22-55] $(\mathrm{p}=0.016))$.

\section{Follow up results}

Figures 1A and 1B illustrate the base-line findings and outcomes. During a median FU of 56 months (1-90 months) 8 patients (30\%) with LGE experienced an adverse event. All adverse cardiac events occurred in patients who had presented with cardiac symptoms. A 57 year-old female (NYHA class 3, non-dilated, scarred LV, LV LGE 32\%, non-sustained VTs (nsVT) at rest) awaiting elective ICD implantation experienced SCD, one patient was admitted because of CCF, and six patients had appropriate ICD therapy/discharge for sVT/VF. The average annualized ICD therapy rate was $11.1 \%$. Three layer confluent transmural LGE predicted arrhythmic events $(\mathrm{p}=0.018)$ and the composite primary endpoint $(p=0.003)$. VT at inclusion or during FU did not correlate with any specific localization grouping of LGE, such as basal septal LGE. Two patients, one presenting with palpitations, the other with pre-syncopal symptoms, both without LGE $(2 / 57,4 \%)$ or T2 signal, suffered an adverse event, respectively sVT after 10 months, and PM implantation for $3^{\text {rd }}$ degree AVB after 6 months. End-points occurred up to 80 months after baseline CMR. Three patients died of non-cardiac conditions, respectively sepsis, respiratory failure due to extensive pulmonary fibrosis and malignancy. None of the patients with non-specific symptoms, such as fatigue, who had been routinely evaluated for cardiac involvement and had LGE (7/59 (12\%), LV LGE 5.9 $\pm 3.8 \%$ ) developed adverse cardiac events during follow up. 


\section{Predictors of events}

Table 4 demonstrates the predictors of adverse events in our study. RV, LV and biventricular LGE were the strongest predictors for adverse events. Six of eight arrhythmic endpoints occurred in patients with LVEF $>35 \%$. Univariate Cox regression analysis found RV LGE 8.71 (95\% CI 1.90-23.81), LV LGE (HR 9.22 (95\% CI 1.96-43.45), and biventricular LGE (HR 12.09 (95\% CI 3.43-42.68), to be the strongest predictors of the composite primary end-point. Multivariate Cox regression analysis, including CCF, LV LGE, biventricular LGE, any LGE, LGE with systolic ventricular dysfunction, LV dilation, and LV or RV systolic dysfunction, revealed biventricular LGE to be the best independent predictors of the composite primary and secondary end-points at follow up $(\mathrm{p}<0.001$, HR 10.2, 95\% CI 2.92-35.71, respectively $p=0.001$, HR 6.80, 95\% CI 2.19-21.28). None of the other parameters reached statistical significance. The Kaplan Meier event-free survival curves found RV LGE, LV LGE (log rank (Mantel Cox) p = 0.001) and biventricular LGE (log rank (Mantel Cox) $\mathrm{p} \leq 0.001)$ the strongest predictors for the composite primary endpoint. RV LGE and biventricular LGE were the strongest predictors for the composite secondary end-point (log rank (Mantel Cox) $\mathrm{p} \leq$ 0.001). (Figure 2)

In the present patient population ROC curve analysis indicated that $\% \mathrm{LV}$ LGE had the modest ability to predict the composite primary adverse outcome (AUC $=0.77,95 \% \mathrm{CI} 0.58$ to 0.95$)$. A cut-off level of $7 \%$ LV LGE best predicted combined adverse cardiac outcomes, with a sensitivity of $70 \%$, and a specificity of $85 \%$. The test's positive and negative predictive values were $39 \%$ and $95 \%$ respectively. Figures 3.1-4 demonstrate LGE in symptomatic patients with and without adverse events during FU.

One appropriate shock would be delivered for every 2.5 implanted ICDs in sarcoidosis patients, when based on the combination of cardiac symptoms, abnormal ECG and/or rhythm monitoring, and biventricular LGE or LGE $>7 \%$ of LV mass.

\subsection{Discussion}

Our findings demonstrate that the extent and distribution of myocardial LGE in a cohort of predominantly middle-aged Caucasian women with chronic pulmonary sarcoidosis, correlates with ventricular volumes, systolic impairment, and most significantly predicts adverse (arrhythmic) outcome. Our study is the first to prospectively detail RV assessment, and include $\mathrm{T} 2$-weighted assessment in the majority of patients. (Table 1)

Recently systolic RV impairment, multi-focal RV LGE, and active RV granulomatous inflammation in CS were associated with LV LGE, sVT and death. (2,7,11,12,21,22,24) Our study is the first to demonstrate the direct relationship between RV LGE, RV volumes, RV systolic impairment, sVT, and death of all cause. In the absence of data on VT morphology it remains unclear whether the prognostic relevance of RV LGE is related to more extensive biventricular arrhythmogenic substrate or whether RV LGE itself is particularly arrhythmogenic. $(20,24)$

None of our patients with asymptomatic LGE suffered adverse cardiac events, while the majority of arrhythmic end-points (75\%) occurred in patients with LVEF $>35 \%$. Our 
findings support the findings of several recent studies which evaluated the risk of SCD in CS patients and also recorded appropriate ICD therapy in a large proportion of patients with LVEF > 35\%. $(4,7,12,19)$

The risk of VT and SCD in CS seems primarily related to amount and distribution of granulomas and scar, and not the systolic function. Arrhythmic substrate imaging with DECMR and/or PET determines the risk of arrhythmic events more accurately than LVEF. $(2,4,7,12,19,20,22,24)$ The negative predictive value of LGE negative DECMR is excellent, with only one patient with LV LGE $<8 \%$ developing an arrhythmic event after 10 months of follow up. In the absence of prospective data, it seems prudent to follow asymptomatic sarcoidosis patients up with ECG and cardiac ultrasound, and evaluate suspected or confirmed CS with PET and/or DECMR. (19) Ongoing research will determine the optimal study follow-up interval. By reserving DECMR for sarcoidosis patients with cardiac symptoms, and/or abnormalities on basic assessment (resting ECG, ambulatory rhythm monitoring, and/or cardiac ultrasound), and implanting ICDs in patients with LGE $\geq 7 \%$, costs could be contained and benefit optimized - in our study one appropriate shock was delivered for every 2.5 implanted devices. Our appropriate annual ICD therapy/ discharge rate of $11.1 \%$ compares to the $8.6-14.5 \%$ previously reported. (19) The value of routine programmed electrical stimulation (PES) as part of risk stratification in patients without palpitations or pre/syncopal events, with LGE and LVEF $>35 \%$ remains to be determined. (19)

Our study supports the recommendations of the 2014 HRS guidelines concerning the use of DECMR and device implantation. (19)

The prognostic studies summarized in Table 1 generally report LGE in 25-30\% of unselected patients cohorts, and uniformly confirm the relationship between the extent of LGE and adverse outcome. Our study confirmed the favorable prognosis of small, asymptomatic myocardial scars as previously reported. $(\mathbf{7 , 9 , 1 2 , 1 3 , 1 4 )}$ The remarkable difference between our conclusions and those of Patel et al, who reported small asymptomatic scar in patients with mildly impaired systolic ventricular functions to be a strong risk factor for adverse events, could partly be explained by a difference in patient population and the distribution of LGE. (4) Patel's cohort mainly consisted of African American women with RV LGE in 67\%, a potentially high risk scenario. Long-term outcomes in pts with CS have markedly improved due to modern heart failure management, including device therapy and arrhythmia ablation in selected patients. $(\mathbf{1 0}, \mathbf{1 1}, \mathbf{2 4 , 2 6 , 2 7 )}$ Current annual mortality rates range from $0-4.2 / 100$ pts compared to $7.5-12 / 100$ pts as previously reported by Yazaki (2001) and Fleming (1987). (19) Our study is the first to include data on T2-weigthed edema imaging. Active myocardial inflammation may increase arrhythmogenicity, but conflicting data exist concerning the efficacy of current immune-suppressive and anti-arrhythmic treatment in actually improving long-term outcomes in patients with active disease. $(\mathbf{1 1}, 12,19,20,24,26,27)$ The presence of increased T2 signal in our cohort, managed with corticosteroids and methotrexate, did not correlate with adverse events during follow up. The accuracy for detecting active granulomatous sarcoidosis with T2-weighted Spin Echo sequences is however suboptimal, and we may well have underestimated inflammatory changes. (3) T2-mapping and FDG-PET have shown promise in diagnosing active disease 
and guiding immune-suppressive management and have replaced spin echo assessment. $(2,12,23,24)$

\subsection{Study limitations:}

Our study is limited by relatively small number of, predominantly Caucasian, patients and few events. African-American and Japanese populations generally have higher rates of cardiac involvement with more extensive myocardial involvement, and possibly a more malignant course. $(4,7,12,27)$ Intermittent ambulatory rhythm monitoring may have missed sVT. However the long-term outcome in our cohort still remained favorable. T1/T2 mapping would have increased the detection of interstitial fibrosis and inflammation and have potentially increased prognostic accuracy. $(23,28)$

\subsection{Conclusions}

LGE is the strongest, independent CMR predictor of future adverse cardiac events in sarcoidosis patients. RV involvement in addition to LV LGE increases risk of adverse cardiac outcomes and death of all causes. DECMR should ideally be performed in sarcoidosis patients with cardiac symptoms. Asymptomatic patients with LGE $<8 \%$ of LV mass and mildly impaired LVEF may not benefit from device therapy, and be monitored. Future prospective studies will help determine the timing of DECMR studies. Current medical management including device therapy has improved survival in this condition. $(10,19,22,27)$

\subsection{Perspectives}

Biventricular myocardial LGE is the strongest, independent CMR predictor of future adverse cardiac events in sarcoidosis patients. DECMR should ideally be performed in every sarcoidosis patient with cardiac symptoms and/or abnormal basic assessment. The presence of symptoms and extent of LGE, and not predominantly LVEF will guide the managing clinician in when to implant an ICD. Asymptomatic Caucasian patients with limited LGE $<8 \%$ of LV mass, and preserved LVEF, may not benefit from device therapy, and can be safely observed. Future research will focus on comprehensive diagnostic and prognostic strategies which will include serological markers of disease activity and heart failure, LGE characteristics, and pre- and post contrast T1/T2 mapping to evaluate interstitial fibrosis and extracellular matrix volume. Hybrid PET-CMR imaging may optimize detection and management of active inflammation. DECMR may help plan VT ablation and potentially increase success rates and outcomes. 


\subsection{Acknowledgements}

We are greatly indebted to dr J. Schreurs (The Hague) who enrolled patients and provided us kindly with the follow up findings. The expert statistical advise of $\mathrm{dr}$ P. Nelemans of the Department of Epidemiology at Maastricht University Medical Centre is greatly valued. We gratefully acknowledge Pie Medical Imaging (Maastricht, The Netherlands) which provided the CMR post-processing software.

\section{Disclosures}

None. 


\section{References}

1. Youssef G, Beanlands RSB, Birnie DH, Nery PB. Cardiac sarcoidosis: applications of imaging in diagnosis and directing treatment. Heart 2011;97:2078-2087.

2. Blankstein R, Osborn M, Naya M, Waller A, Ki CK, Murthy VL, Kazemian P, Kwong RY, Tokuda M, Skali H, Padera R, Hainer J, Stevenson WG, Dorbala S, Di Carli MF. Positron emission tomography enhances prognostic assessments of patients with suspected cardiac sarcoidosis. J Am Coll Cardiol 2014:63:329-336.

3. Cheong BY, Muthupillai R, Nemeth M, Lambert B, Dees D, Huber S, Castriotta R, Flamm SD. The utility of delayed-enhancement magnetic resonance imaging for identifying non-ischemic myocardial fibrosis in asymptomatic patients with biopsy-proven systemic sarcoidosis. Sarcoidosis Vasc Diffuse Lung Dis 2009;26:39-46.

4. Patel MR, Cawley PJ, Heitner JF, Klem I, Parker MA, Jaroudi WA, Meine TJ, White JB, Elliott MD, Kim HW, Judd RM, Kim RJ. Detection of myocardial damage in patients with sarcoidosis. Circulation 2009;120:1969-1977.

5. Shafee MA, Fukuda K, Wakayama Y, Nakano M, Kondo M, Hasebe Y, Kawana A, Shimokawa H. Delayed enhancement on cardiac magnetic resonance imaging is a poor prognostic factor in patients with cardiac sarcoidosis. J Cardiol 2012;60:448-453.

6. Greulich S, Deluigi CC, Gloekler S, Wahl A, Zürn C, Kramer U, Nothnagel D, Bültel H, Schumm J, Grün S, Ong P, Wagner A, Schneider S, Nassenstein KM, Sechtem U, Bruder O, Mahrhold H. CMR Imaging predicts death and other adverse events in suspected cardiac sarcoidosis. J Am Coll Cardiol Img 2013;6:501-511.

7. Crawford T, Mueller G, Sarsam S, Prasitdumrong H, Chaiyen N, Gu X, Schuller J, Kron J, Nour KA, Cheng A, Ji SY, Feinstein S, Gupta S, Lig K, Sinno M, Abu-Hashih S, Al-Mallah M, Sauer WH, Ellenbogen K, Morady $\mathrm{F}$, Bogun $\mathrm{F}$. Magnetic resonance imaging for identifying patients with cardiac sarcoidosis and preserved or mildly reduced left ventricular function at risk of ventricular arrhythmias. Circ Arrhythm Electrophysiol 2014;7:1109-1115.

8. Ise T, Hasegawa T, Morita Y, Yamada N, Funada A, Takahama H, Amaki M, Kanzaki H, Okamura H, Kamakura S, Shimizu W, Anzai T, Kitakaze M. Extensive late gadolinium enhancement on cardiovascular magnetic resonance predicts adverse outcomes and lack of improvement in LV function after steroid therapy in cardiac sarcoidosis. Heart 2014;100:1165-1172.

9. Nagai T, Kohsaka S, Okuda S, Anzai T, Asano K, Fukuda K. Incidence and prognostic significance of myocardial late gadolinium-enhancement in sarcoidosis patients without cardiac manifestation. Chest 2014;146(4):1064-1072.

10. Nadel J, Lancefield T, Voskoboinik A, Taylor AJ. Late gadolinium enhancement identified with cardiac magnetic resonance imaging in sarcoidosis patients is associated with long-term ventricular arrhythmia and sudden cardiac death. Eur Heart J Cardiovasc Imaging 2015;16:1634-1641.

11. Ekström K, Lehtonen J, Hänninen H, Kandolin R, Kivistö, Kupari M. Magnetic resonance imaging as a predictor of survival free of life-threatening arrhythmias and transplantation in cardiac sarcoidosis. $\mathrm{J}$ Am Heart Assoc 2016;5:e003040

12. Murtagh G, Laffin LJ, Beshai JF, Maffessanti F, Bonham CA, Patel AV, Yu Z, Addetia K, Mor-Avi V, Moss JD, Hogarth DK, Sweiss NJ, Lang RM, Patel AR . Prognosis in myocardial damage in sarcoidosis patients with preserved left ventricular ejection fraction. Risk stratification using cardiac magnetic resonance. Circ Cardiovasc Imag 2016;9:e003738.

13. Agoston-Coldea L, Kouaho S, Sacre K, . High mass ( $>18 \mathrm{~g})$ of late gadolinium enhancement on CMR imaging is associated with major cardiac events on long-term outcome in patients with biopsy proven extracardiac sarcoidosis. Int J Cardiol 2016;222:950-956.

14. Yasuda M, Iwanaga Y, Kato T, Izumi T, Inuzuka Y, Nakamura T, Miyaji Y, Kawamura T, Ikeguchi S, Inoko M, Kurita T, Miyazaki S. Risk stratification for major adverse cardiac events and ventricular tachyarrhythmia's by cardiac MRI in patients with sarcoidosis. Open Heart 2016;3:e000437. Doi:10.1136/openhrt-2016000437

15. Smedema JP, Snoep G, van Kroonenburgh MPG, van Geuns RJ, Dassen WR, Gorgels T, Crijns HJGM . Evaluation of the accuracy of gadolinium-enhanced cardiovascular magnetic resonance in the diagnosis of cardiac sarcoidosis. J Am Coll Cardiol 2005;45:1683-1690.

16. Rudski LG, Lai WW, Afilalo J, Hua L, Handschumacher MD, Chandrasekaran K, Solomon SD, Louie EK, Schiller NB. Guidelines for the echocardiographic assessment of the right heart in adults: a report from the American Society of Echocardiography endorsed by the European Association of Echocardiography, a reg- 
istered branch of the European Society of Cardiology, and the Canadian Society of Echocardiography. J Am Soc Echocardiogr 2010;23:685-713.

17. Gulati A, Ismail TF, Jabbour A, Alpendurada F, Guha K, Ismail NA, Raza S, Khwaja D, Brown TD, Morarji K, Liodakis E, Roughton M, Wage R, Pakrashi TC, Sharma R, Carpenter JP, Cook SA, Cowie MR, Assomull RG, Pennell DJ, Prasad SK. The prevalence and prognostic significance of right ventricular systolic dysfunction in non-ischemic dilated cardiomyopathy. Circulation 2013;128(15):1623-1636.

18. Epstein AE, DiMarco JP, Ellenbogen KA, et al. American College of Cardiology/American Heart Association Task Force on Practice Guidelines (Writing Committee to Revise the ACC/AHA/NASPE 2002 Guideline Update for Implantation of Cardiac Pacemakers and Antiarrhythmia Devices); American Association for Thoracic Surgery; Society of Thoracic Surgeons. ACC/AHA/HRS 2008 guidelines for device-based therapy of cardiac rhythm abnormalities. Circulation 2008;117:e350-e408.

19. Birnie DH, Sauer WH, Bogun F, Cooper JM, Culver DA, Duvernoy CS, Judson MA, Kron J, Mehta D, Cosedis Nielsen J, Patel AR, Ohe T, Raatikainen P, Soejima K. HRS Expert consensus statement on the diagnosis and management of arrhythmias associated with cardiac sarcoidosis. Heart Rhythm 2014;11(7):13051323.

20. Jefic D, Joel B, Good E, Morady F, Rosman H, Knight B, Bogun F. Role of radiofrequency catheter ablation of ventricular tachycardia in cardiac sarcoidosis: Report from a multi-centre registry. Heart Rhythm 2009;6:189-195.

21. Schuller JL, Zipse M, Crawford T, Bogun F, Beshai J, Patel AR, Sweiss NJ, Nguyen DT, Aleong RG, Varosy PD, Weinberger HD, Sauer WH . Implantable cardioverter defibrillator therapy in patients with cardiac sarcoidosis. J Cardiovasc Electrophysiol 2012;23:925-929.

22. Patel AR, Klein MR, Chandra S, Spencer KT, Decara JM, Lang RM, Burke MC, Garrity ER, Hogarth DK, Archer SL, Sweiss NJ, Beshai JF. Myocardial damage in patients with sarcoidosis and preserved left ventricular systolic function: an observational study. Eur J Heart Fail 2011;13:1231-1237.

23. Crouser ED, Ono C, Tran T, He X, Raman SV. Improved detection of cardiac sarcoidosis using magnetic resonance with myocardial T2 mapping. Am J Resp Crit Care Med 2014;189:109-112

24. Muser D, Santangeli P, Patahk RK, Castro SA, Liang JJ, Magnani S, Hayashi T, Garcia FC, Hutchinson MD, Supple GE, Frankel DS, Riley MP, Lin D, Schaller RD, Desjardins B, Dixit S, Callans DJ, Zado ES, Marchlinski FE. Long-term outcomes of catheter ablation of ventricular tachycardia in patients with cardiac sarcoidosis. Circ Arrhythm Electr 2016;9(8):e004333.

25. Smedema JP, van Geuns RJ, Ainslie G, Ector J, Heibuchel H, Crijns HJGM. Right ventricular involvement in cardiac sarcoidosis demonstrated with cardiac magnetic resonance. ESC Heart Failure 2017, in print.

26. Kandolin R, Lehtonen J, Airaksinen J, Vihinen T, Miettinen H, Kaikkonen K, Hataaja P, Kerola T, Kupari M. Cardiac sarcoidosis. Epidemiology, characteristics, and outcome over 25 years in a nationwide study. Circulation 2015;131:624-1632.

27. Zhou Y, Lower EE, Li H, Farhey Y, Baughman RP. Cardiac sarcoidosis: the impact of age and implanted devices on survival. Chest (2016), doi:10.1016/ j.chest.2016.08.1457.

28. Greulich S, Kitterer D, Latus J, Aguor E, Steubing H, Kaesemann P, Patrascu A, Greiser A, Groeninger S, Mayr A, Braun N, Alscher MD, Sechtem U, Mahrhold H. Comprehensive cardiovascular magnetic resonance assessment $\mathrm{n}$ patients with sarcoidosis and preserved left ventricular ejection fraction. Circ Cardiovasc Imaging 2016;9:e005022.doi:10.1161/CIRCIMAGING.116. 005022 


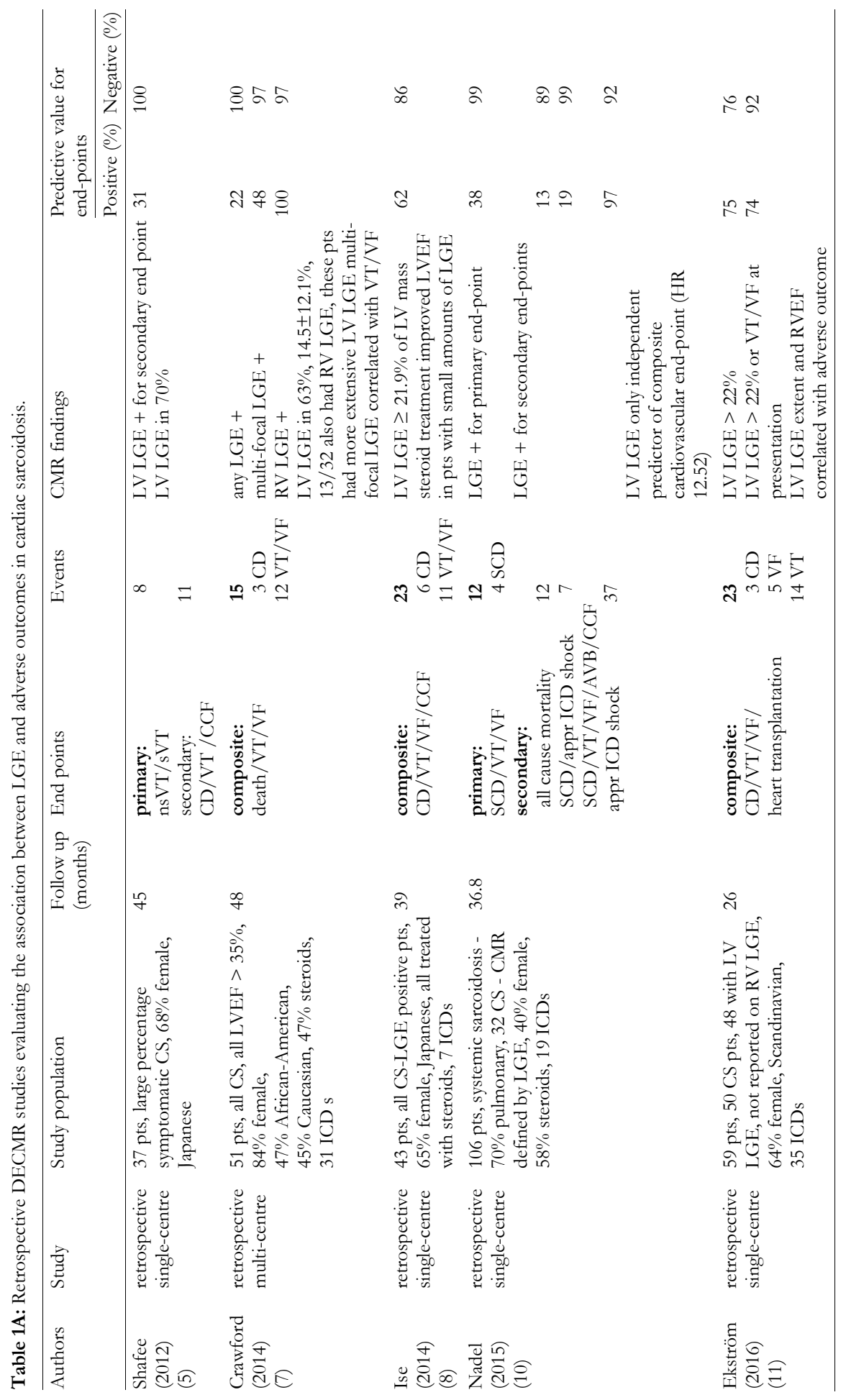




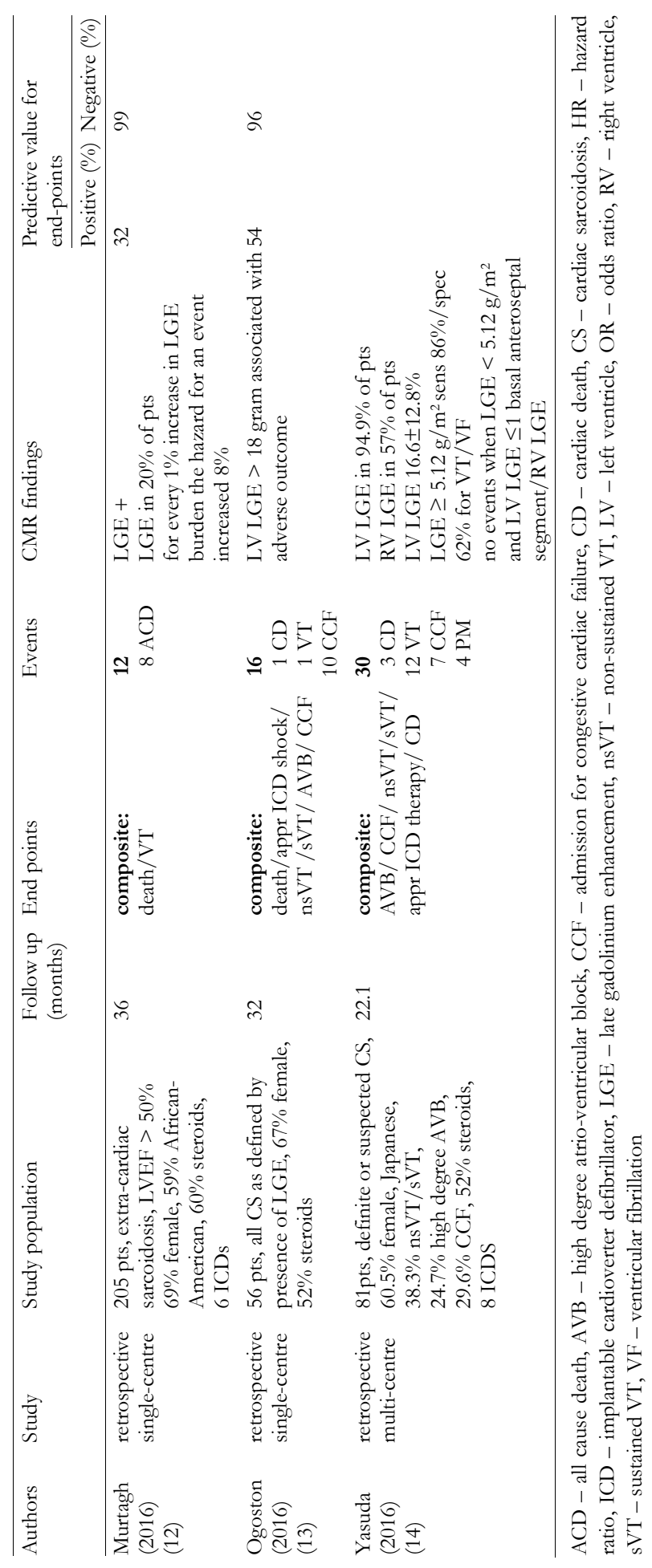




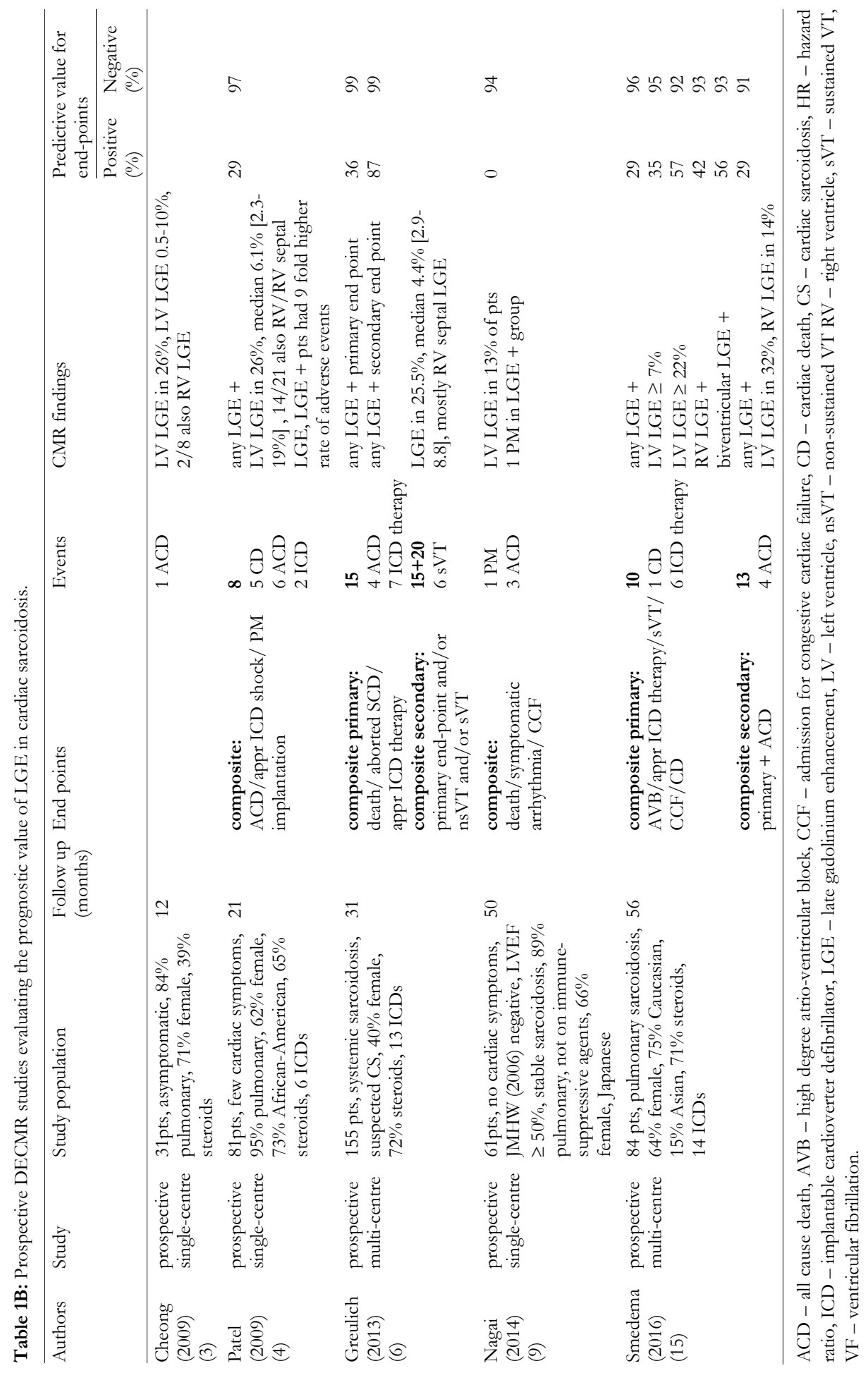


Table 2: Patients baseline characteristics.

\begin{tabular}{|c|c|}
\hline All patients with follow up & $84(100)$ \\
\hline Female & $54(64)$ \\
\hline Caucasian & $63(75)$ \\
\hline Age, years & $53.3 \pm 9.8$ \\
\hline Diabetes Mellitus & $3(4)$ \\
\hline Hypertension & $7(8)$ \\
\hline Cardiac presentation & $25(30)$ \\
\hline Syncope & $4(5)$ \\
\hline Palpitations & $9(11)$ \\
\hline Clinical congestive heart failure & $8(10)$ \\
\hline Dyspnea NYHA class 0 & $50(59)$ \\
\hline NYHA class 1-2 & $29(35)$ \\
\hline NYHA class $3-4$ & $5(6)$ \\
\hline Sustained Ventricular Tachycardia & $10(12)$ \\
\hline Aborted Sudden Cardiac Death & $1(1)$ \\
\hline Chest discomfort & $3(4)$ \\
\hline Abnormal electrocardiogram & $26(31)$ \\
\hline Pulmonary Arterial Hypertension & $14(17)$ \\
\hline \multicolumn{2}{|l|}{ Medication at any time } \\
\hline Steroids & $60(71)$ \\
\hline Methotrexate & $6(7)$ \\
\hline Loop diuretics & $10(12)$ \\
\hline Spironolactone & $10(12)$ \\
\hline Ace inhibitors/ATIIRB & $11(13)$ \\
\hline Beta blockers & $13(16)$ \\
\hline Amiodarone & $15(18)$ \\
\hline \multicolumn{2}{|l|}{ CMR imaging parameters } \\
\hline LVEF, $\%$ & 60 [14-84] \\
\hline Impaired LVEF ( $\leq 50 \%)$ & $16(19)$ \\
\hline Impaired LVEF with LGE & $13(15)$ \\
\hline Impaired RVEF ( $\leq 45 \%)$ & $19(18)$ \\
\hline LVEDV, $\mathrm{ml}$ & 112 [88-136] \\
\hline LVEDV index, $\mathrm{ml} / \mathrm{m}^{2}$ & $58[47-70]$ \\
\hline LV dilation & $8(10)$ \\
\hline LV mass, gr & $116[90-142]$ \\
\hline $\mathrm{LV}$ mass index, $\mathrm{gr} / \mathrm{m}^{2}$ & $64[44-84]$ \\
\hline LVH & $22(26)$ \\
\hline LV LGE present & $27(32)$ \\
\hline LV LGE, gr & $20[8-45]$ \\
\hline LV LGE, $\%$ of LV mass & $15[6-33]$ \\
\hline RVEF, $\%$ & $47[40-54]$ \\
\hline RVEDV, $\mathrm{ml}$ & $140[97-183]$ \\
\hline RVEDV index, $\mathrm{ml} / \mathrm{m}^{2}$ & 78 [58-98] \\
\hline $\mathrm{RV}$ dilation & $13(15)$ \\
\hline RV mass, gr & $43[34-52]$ \\
\hline $\mathrm{RV}$ mass index, $\mathrm{gr} / \mathrm{m}^{2}$ & $23[18-28]$ \\
\hline RVH & $11(11)$ \\
\hline RV LGE present & $12(14)$ \\
\hline T2 positive & $10 / 69(14)$ \\
\hline Follow up, months & $59.0 \pm 22.2[3-108]$ \\
\hline
\end{tabular}




\begin{tabular}{lc}
\hline Cardiac events during follow up & $10(12)$ \\
Cardiac death & $1(1)$ \\
Admission for congestive heart failure & $1(1)$ \\
Appropriate ICD therapy & $7(8)$ \\
Atrio-ventricular block, Pacemaker implantation & $1(1)$ \\
Time to events, months & $6(1-80)$
\end{tabular}

Values are $\mathrm{n}(\%)$, median $[\mathrm{IQR}]$, or mean \pm SD. CMR = cardiac magnetic resonance; EDV = end-diastolic volume; ICD = implantable cardioverter-defibrillator; LGE = late gadolinium enhancement; $L V=$ left ventricle; LVEDV = left ventricular end-diastolic volume; $\mathrm{LVEF}=$ left ventricular ejection fraction; $\mathrm{RV}=$ right ventricle; $\mathrm{RVEF}=$ right ventricular ejection fraction; $\mathrm{RVH}=$ right ventricular hypertrophy 
Table 3: Characteristics of patients with and without LGE.

\begin{tabular}{|c|c|c|c|c|}
\hline & $\begin{array}{l}\text { LGE - } \\
\mathrm{n}=56\end{array}$ & $\begin{array}{l}\mathrm{LGE}+ \\
\mathrm{n}=28\end{array}$ & $\mathrm{p}$ Value & OR $(95 \% \mathrm{CI})$ \\
\hline Female & $19(34)$ & $11(39)$ & 0.638 & \\
\hline Caucasian & $42(75)$ & $21(75)$ & 1 & \\
\hline Age, years & $52.4 \pm 10.1$ & $55.3 \pm 8.9$ & 0.37 & \\
\hline $\begin{array}{l}\text { Cardiac presentation } \\
\text { Syncope } \\
\text { Palpitations } \\
\text { Clinical congestive heart failure } \\
\text { Sustained Ventricular Tachycardia } \\
\text { Aborted Sudden Cardiac Death } \\
\text { Chest discomfort }\end{array}$ & $\begin{array}{l}5(9) \\
1(2) \\
2(4) \\
2(4) \\
3(5) \\
0 \\
0\end{array}$ & $\begin{array}{l}20(71) \\
3(11) \\
7(26) \\
6(21) \\
7(25) \\
1(4) \\
3(11)\end{array}$ & $\begin{array}{l}<\mathbf{0 . 0 0 1} \\
0.096 \\
\mathbf{0 . 0 0 4} \\
\mathbf{0 . 0 1 2} \\
\mathbf{0 . 0 1 1} \\
0.321 \\
\mathbf{0 . 0 3 1}\end{array}$ & $\begin{array}{l}29.71(8.44-104.60) \\
7(0.69-70.74) \\
9.60(1.84-50.25) \\
7.86(1.47-42.04) \\
6.30(1.483-26.765) \\
1.13(0.99-0.129)\end{array}$ \\
\hline $\begin{array}{ll}\text { Dyspnea } & \text { NYHA 0-2 } \\
& \text { NYHA 3-4 }\end{array}$ & $\begin{array}{l}55(96) \\
2(4)\end{array}$ & $\begin{array}{l}26(93) \\
1(4)\end{array}$ & $\begin{array}{l}0.96 \\
1\end{array}$ & \\
\hline Diabetes Mellitus & $3(5)$ & 0 & 0.548 & \\
\hline Hypertension & $6(11)$ & $1(4)$ & 0.420 & \\
\hline $\begin{array}{l}\text { Medication at any time } \\
\text { Steroids } \\
\text { Methotrexate } \\
\text { Loop diuretics } \\
\text { Spironolactone } \\
\text { Ace inhibitors/ATIIRB } \\
\text { Beta blockers } \\
\text { Amiodarone }\end{array}$ & $\begin{array}{l}36(63) \\
1(2) \\
2(4) \\
2(4) \\
1(2) \\
2(4) \\
2(4)\end{array}$ & $\begin{array}{l}24(86) \\
5(18) \\
8(29) \\
8(29) \\
10(36) \\
11(39) \\
12(43)\end{array}$ & $\begin{array}{l}0.019 \\
0.012 \\
0.001 \\
0.001 \\
<0.001 \\
<0.001 \\
<0.001\end{array}$ & \\
\hline Pulmonary Arterial Hypertension & $5(9)$ & $9(32)$ & 0.010 & $5.100(1.508-17.243)$ \\
\hline $\begin{array}{l}\text { CMR imaging parameters } \\
\text { LVEF \% } \\
\text { LVEDV ml } \\
\text { LVEDV index } \mathrm{ml} / \mathrm{m}^{2} \\
\mathrm{LV} \text { mass } \\
\mathrm{LV} \text { mass index } \mathrm{gr} / \mathrm{m}^{2} \\
\mathrm{LVH} \\
\mathrm{LV} \text { dilation } \\
\mathrm{LVEF} \leq 50 \% \\
\mathrm{RVH} \\
\mathrm{RV} \text { mass } \\
\mathrm{RV} \text { mass index } \mathrm{gr} / \mathrm{m}^{2} \\
\mathrm{RV} \leq 45 \% \\
\text { RVEF } \% \\
\text { RVEDV ml } \\
\text { RVEDV index } \mathrm{ml} / \mathrm{m}^{2} \\
\text { T2 positive }\end{array}$ & $\begin{array}{l}64[50-70] \\
111[91-131] \\
55[48-62] \\
114[94-134] \\
64[44-84] \\
26(46) \\
3(5) \\
3(5) \\
4(7) \\
39[32-46] \\
22[16-28] \\
6(11) \\
48[42-54] \\
185[143-227] \\
81[61-101] \\
1 / 45(2)\end{array}$ & $\begin{array}{l}55[49-72] \\
132[92-172] \\
71[49-93] \\
122[92-152] \\
64[43-85] \\
15(54) \\
11(39) \\
13(48) \\
7(25) \\
42[35-49] \\
23[20-26] \\
13(48) \\
46[36-56] \\
183[140-226] \\
96[69-123] \\
9 / 24(38)\end{array}$ & $\begin{array}{l}<\mathbf{0 . 0 0 1} \\
\mathbf{0 . 0 2} \\
\mathbf{0 . 0 0 7} \\
0.599 \\
0.363 \\
0.410 \\
<\mathbf{0 . 0 0 1} \\
<\mathbf{0 . 0 0 1} \\
\mathbf{0 . 0 2 9} \\
0.753 \\
0.964 \\
\mathbf{0 . 0 1 1} \\
0.288 \\
0.084 \\
\mathbf{0 . 0 2 8} \\
<\mathbf{0 . 0 0 1}\end{array}$ & $\begin{array}{l}11.216(2.796-44.988) \\
11.842(2.342-59.879) \\
4.333(1.147-16.366)\end{array}$ \\
\hline Follow up, months & $52.4 \pm 22.1$ & $61.7 \pm 21.5$ & 0.62 & \\
\hline Events during follow up & $2(4)$ & $8(30)$ & 0.001 & \\
\hline Time to event, months & $8(6-10)$ & $4.5(1-80)$ & 0.701 & \\
\hline
\end{tabular}

Values are $\mathrm{n}(\%)$, median $[\mathrm{IQR}]$, or mean \pm SD. $*$ Values are for all patients with sarcoidosis $(84)$. CI $=$ confidence interval; $\mathrm{CMR}=$ cardiac magnetic resonance; $\mathrm{EDV}=$ end-diastolic volume; $\mathrm{ICD}=$ implantable cardioverterdefibrillator; LGE = late gadolinium enhancement; $L V=$ left ventricle; LVEDV = left ventricular end-diastolic volume; $\mathrm{LVEF}=$ left ventricular ejection fraction; $\mathrm{RV}=$ right ventricle; $\mathrm{RVEF}=$ right ventricular ejection fraction; $\mathrm{RVH}=$ right ventricular hypertrophy. 
Table 4: Univariate analysis for association with the primary composite end-point.

\begin{tabular}{|c|c|c|c|c|}
\hline & $\begin{array}{l}\text { No end-points } \\
\mathrm{n}=74\end{array}$ & $\begin{array}{l}\text { Primary } \\
\text { composite } \\
\text { end-point } \\
n=10\end{array}$ & $\mathrm{p}$ Value & OR $(95 \% \mathrm{CI})$ \\
\hline Female & $48(65)$ & $6(60)$ & 0.76 & $0.81(0.21-3.14)$ \\
\hline Caucasian & $56(76)$ & $7(70 \%)$ & 0.70 & $0.75(0.175-3.21)$ \\
\hline Age, years & $53 \pm 10$ & $55 \pm 7$ & 0.66 & \\
\hline Cardiac presentation & $15(20)$ & $10(100)$ & $<0.001$ & $12.44(3.037-50.994)$ \\
\hline Syncope & $2(3)$ & $2(20)$ & 0.02 & $9.00(1.11-72.88)$ \\
\hline Palpitations & $4(5)$ & $5(50)$ & $<0.001$ & $17.50(3.54-86.46)$ \\
\hline Clinical congestive heart failure & $5(7)$ & $3(30)$ & 0.02 & $5.91(1.16-30.15)$ \\
\hline Sustained Ventricular Tachycardia & $6(8)$ & $5(50)$ & $<0.001$ & $11.33(2.54-50.51)$ \\
\hline Aborted Sudden Cardiac Death & $1(1)$ & 0 & 0.71 & \\
\hline Chest discomfort & $3(4)$ & 0 & 0.52 & \\
\hline Dyspnea NYHA class $0-2$ & $72(97)$ & $9(90)$ & 0.24 & \\
\hline NYHA class 3-4 & $2(3)$ & $1(10)$ & 0.24 & $4(0.33-48.66)$ \\
\hline Diabetes Mellitus & $3(4)$ & 0 & 0.52 & \\
\hline Hypertension & $7(9)$ & 0 & 0.31 & \\
\hline \multicolumn{5}{|l|}{ Medication at any time } \\
\hline Steroids & $51(69)$ & $9(90)$ & 0.17 & \\
\hline Methotrexate & $5(7)$ & $1(10)$ & 0.71 & \\
\hline Loop diuretics & $6(8)$ & $4(40)$ & 0.003 & \\
\hline Spironolactone & $6(8)$ & $4(40)$ & 0.003 & \\
\hline Ace inhibitors/ATIIRB & $6(8)$ & $5(50)$ & $<0.001$ & \\
\hline Beta blockers & $5(7)$ & $8(80)$ & $<0.001$ & \\
\hline Amiodarone & $7(9)$ & $8(80)$ & $<0.001$ & \\
\hline ECG abnormalities at presentation & $19(26)$ & $7(70)$ & 0.004 & $6.75(1.59-28.78)$ \\
\hline Pulmonary Hypertension & $9(12)$ & $5(50)$ & 0.039 & $4.24(1.133-15.833)$ \\
\hline \multicolumn{5}{|l|}{ CMR imaging parameters } \\
\hline LVEF \% & 55 [48-62] & $50[35-65]$ & 0.01 & \\
\hline LVEDV ml & $111[88-134]$ & $139[107-171]$ & 0.02 & \\
\hline LVEDV index $\mathrm{ml} / \mathrm{m}^{2}$ & $58[38-78]$ & $81[64-98]$ & 0.02 & \\
\hline $\mathrm{LVEF}<50 \%$ & $11(15)$ & $5(50)$ & 0.01 & $5.36(1.33-21.68)$ \\
\hline $\mathrm{LVEF} \leq 35 \%$ & $3(4)$ & $2(20)$ & 0.06 & $5.58(0.81-38.60)$ \\
\hline LV dilation & $5(7)$ & $3(30)$ & 0.02 & $5.83(1.44-29.72)$ \\
\hline LVH & $17(23)$ & $5(50)$ & 0.12 & \\
\hline LV mass & $106[84-128]$ & $189[151-227]$ & 0.004 & \\
\hline LV LGE present & $12(4-74)$ & $49[32-66]$ & $<0.001$ & $11.58(2.26-59.39)$ \\
\hline LV LGE, $\%$ of LV & $14[3-36]$ & $28[20-36]$ & $<0.001$ & \\
\hline LV LGE with systolic impairment & $8(11)$ & $5(50)$ & 0.001 & $8.25(1.95-34.84)$ \\
\hline RV LGE present & $6(8)$ & $6(60)$ & 0.002 & $9.29(2.350-36.696)$ \\
\hline Biventricular LGE & $5(7)$ & $5(50)$ & 0.004 & $10.47(2.323-47.171)$ \\
\hline RVH & $7(9)$ & $4(40)$ & 0.062 & $4.10(0.989-16.691)$ \\
\hline RV mass & $42[33-51]$ & $50[38-62]$ & 0.305 & \\
\hline $\mathrm{RV}$ mass index $\mathrm{gr} / \mathrm{m}^{2}$ & $22[17-27]$ & $25[19-31]$ & 0.1993 & \\
\hline RV dysfunction & $14(19)$ & $5(50)$ & 0.042 & $3.82(1.101-13.285)$ \\
\hline RVEF \% & $47[41-53]$ & 47 [32-62] & 0.793 & \\
\hline RVEDV & 140 [98-182] & 137 [93-187] & 0.785 & \\
\hline RVEDV index $\mathrm{ml} / \mathrm{m}^{2}$ & 77 [57-97] & 84 [61-107] & 0.431 & \\
\hline T2 positive & 7/52 (13) & $3 / 7(43)$ & 0.13 & $3.18(0.67-15.24)$ \\
\hline Follow up, months & $53 \pm 20$ & $71 \pm 33$ & 0.01 & \\
\hline Time to event, months & & $14.3(1-80)$ & & \\
\hline
\end{tabular}

Values are $\mathrm{n}(\%)$, median $[\mathrm{IQR}]$, or mean $\pm \mathrm{SD}$. CI $=$ confidence interval; $\mathrm{CMR}=$ cardiac magnetic resonance; $\mathrm{EDV}=$ end-diastolic volume; ICD = implantable cardioverter-defibrillator; LGE = late gadolinium enhancement; $\mathrm{LV}=$ left ventricle; $\mathrm{LVEDV}=$ left ventricular end-diastolic volume; $\mathrm{LVEF}=$ left ventricular ejection fraction; $\mathrm{RV}$ $=$ right ventricle; $\mathrm{RVEF}=$ right ventricular ejection fraction; $\mathrm{RVH}=$ right ventricular hypertrophy. 
84 pts with pulmonary sarcoidosis

Baseline investigations: 12 -lead resting ECG, a mbulatory rhythm monitoring, cardiac ultrasound, DECMR

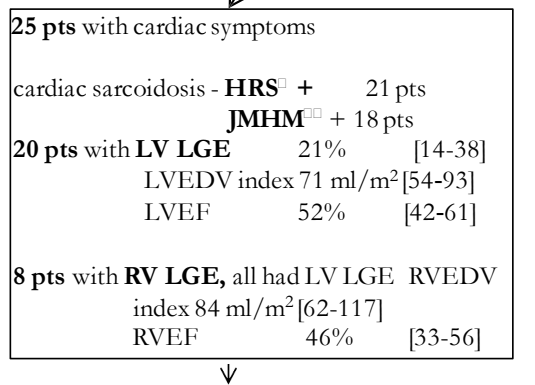

RV EMBs in 9 pts -2 positive for CS

PES in 16 pts - 8 pts sustained VT -3 pts ablated 2 pts AVNRT - ablated

10 ICDs/4 ICD-Bivent PM and 2 PMs implanted

\begin{tabular}{|l|}
$\downarrow$ \\
Follow up 54 [24-84] months \\
\hline
\end{tabular}

59 pts without cardiac symptoms

$$
\begin{aligned}
& \text { cardiac sarcoidosis - HRS }+\quad 8 \text { pts } \\
& \text { JMHW }+0 \text { pts } \\
& 7 \text { pts with LV LGE } \quad 6 \% \quad[4-8] \\
& \text { LVEDV index } 54 \mathrm{ml} / \mathrm{m}^{2} \text { [48-63] } \\
& \text { LVEF } \quad 62 \% \quad \text { [57-68] }
\end{aligned}
$$

4 pts with RV LGE, 2 only with RV LGE + RVEDV index $71 \mathrm{ml} / \mathrm{m}^{2}$ [53-91] RVEF $\quad 47 \% \quad$ [43-52] no EMBs taken PES in 3 pts - negative

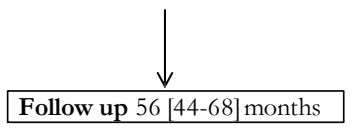

4-6 monthly FU by Cardiology and Respiratory Out-Patient Departments, General Practitioner FU assessment with 12-lead resting ECG, ambulatory rhythm monitoring, cardiac ultrasound

10 cardiac events

1 - SCD at 3 months - biventricular LGE, LV LGE $32 \%$

1 - $3^{\text {rd }}$ degree AVB/PM implantation at 6 months - no LGE

1 - admission for CCF at 15 months - LV LGE $35 \%$

7 - appropriate ICD therapy for VT/VF at 1-80 months - 4 biventricular LGE, LV LGE 21\% [14-33]

\section{Programmed Electrical Stimulation:}

6/8 (75\%) PES with inducible sustained VT had arrhythmic end-point - i.e.VT/VF

1/11 (9\%) PES without inducible VT had arrhythmic end-point - sustained VT at 10 months - no LGE

PPV of PES + for arrhythmic end point: $75 \%$, NPV of PES - for arrhythmic end point $91 \%$,

PES + for sustained VT correlated with trans-mural LGE $(\mathrm{p}=0.018)$, and impaired LVEF $<40 \%(\mathrm{p}=0.018)$,

LVEF in PES + compared to PES - respectively 38\% [20-56] and 61\% [52-70], $\mathrm{p}=0.012$

RVEF in PES + compared to PES - respectively $47 \%[40-54]$ and $51 \%[42-60], \mathrm{p}=0.566$

Figure 1A: Flow diagram of the baseline findings and adverse events during follow up included in the primary composite end-point up in 84 sarcoidosis patients.

HRS - Heart Rhythm Society 2014 criteria for the diagnosis of cardiac sarcoidosis (19)

JMHW - Japanese Ministry of Health and Welfare 2007 criteria for the diagnosis of cardiac Sarcoidosis (1) 


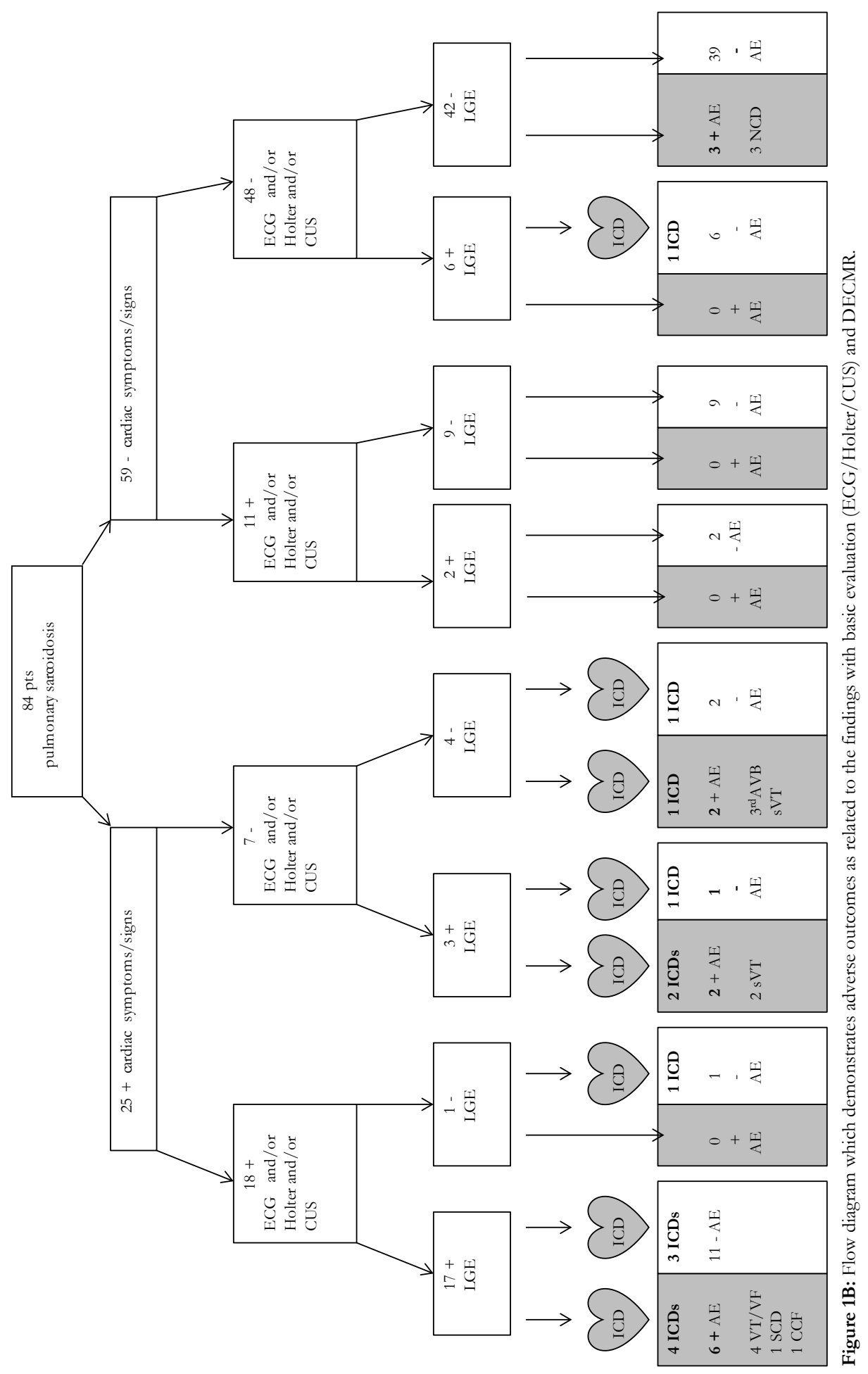




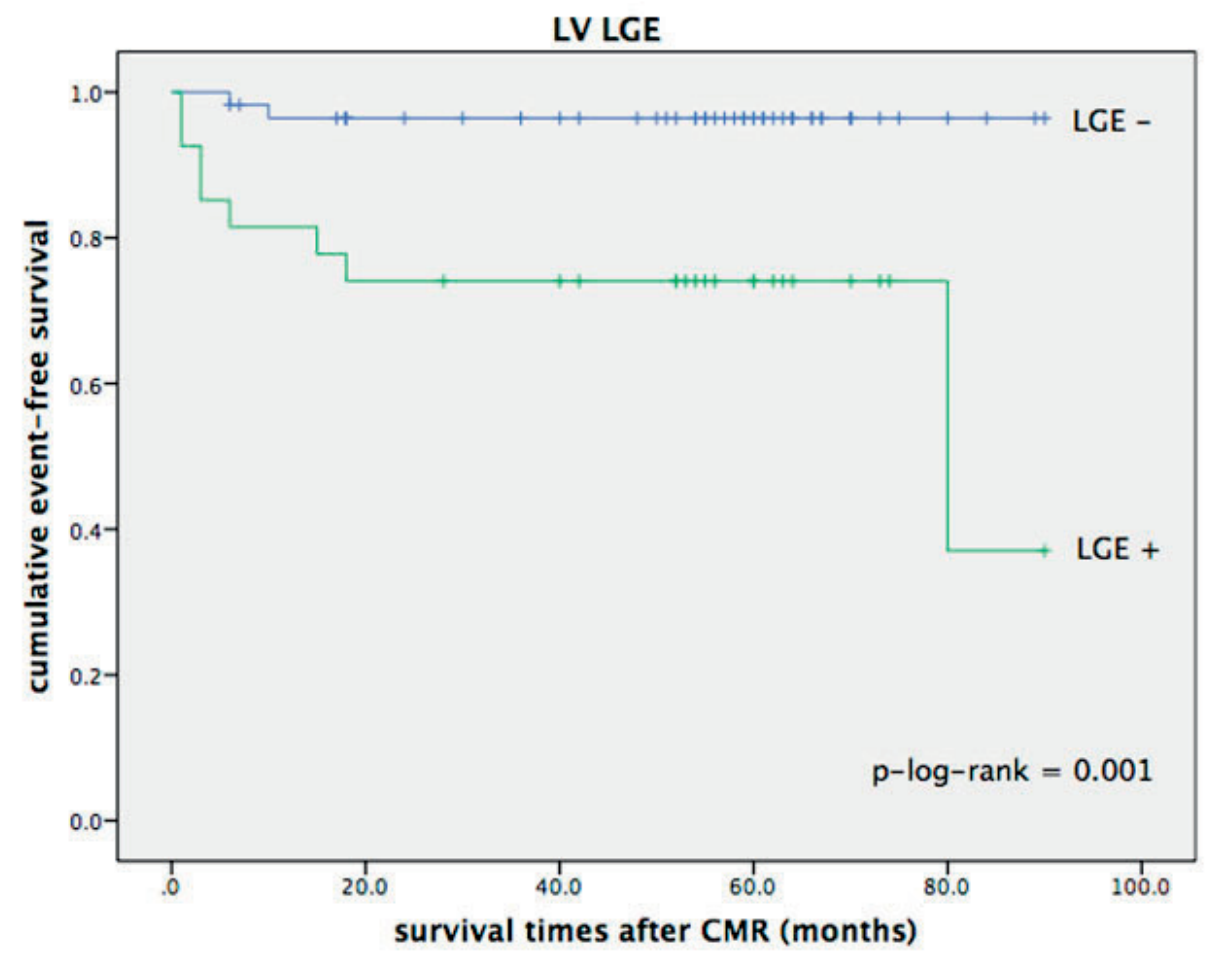

Patient at risk

\begin{tabular}{llllll}
\hline Months & 0 & 20 & 40 & 60 & 80 \\
\hline No LGE & 57 & 45 & 40 & 21 & 3 \\
LV LGE & 26 & 20 & 18 & 8 & 1 \\
\hline
\end{tabular}

Figure 2A: Kaplan-Meier survival curve for LV LGE (composite primary end point). 


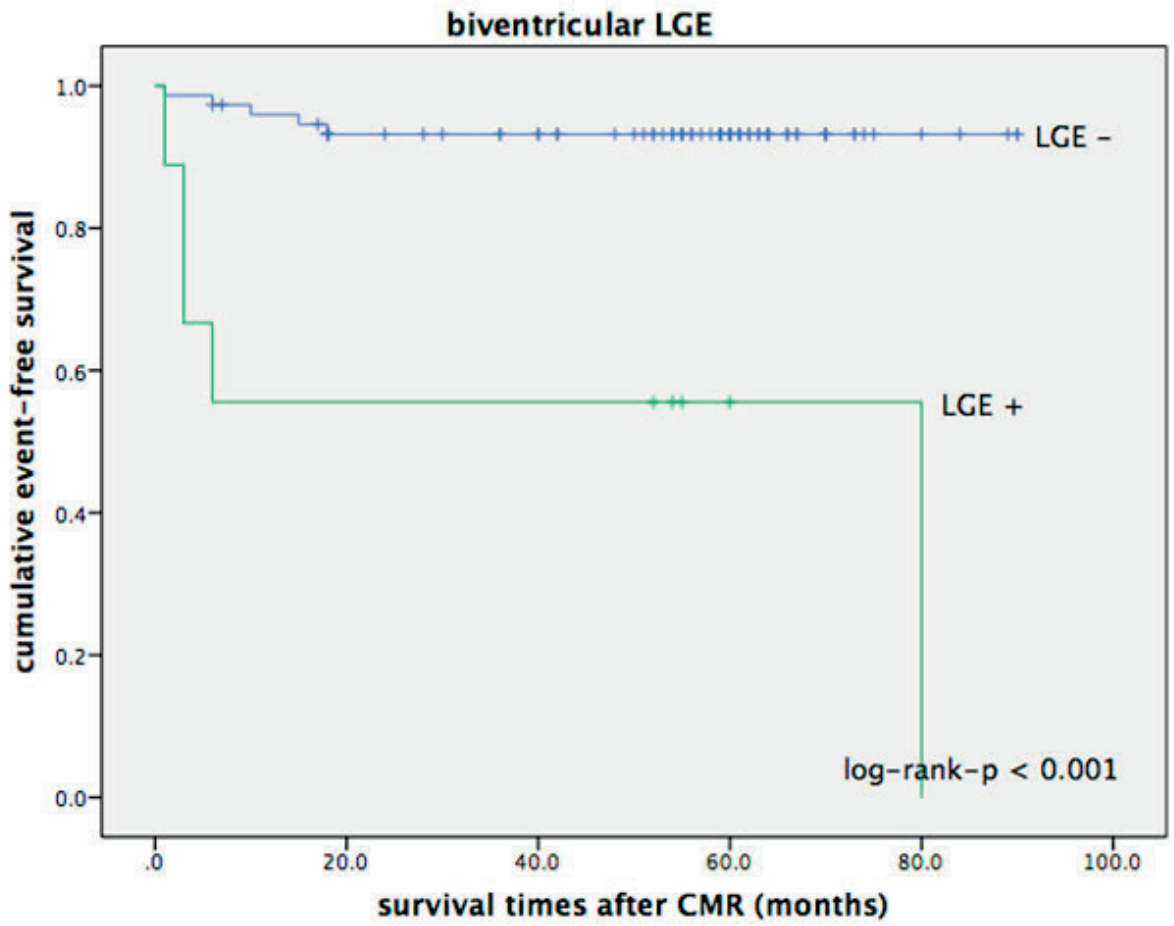

Patients at risk

\begin{tabular}{lrrrrl}
\hline Months & 0 & 20 & 40 & 60 & 80 \\
\hline No LGE & 75 & 55 & 54 & 28 & 4 \\
Bivent LGE & 9 & 5 & 5 & 1 & 0 \\
\hline
\end{tabular}

Figure 2B: Kaplan-Meier survival curve for biventricular LGE (composite primary end point). 


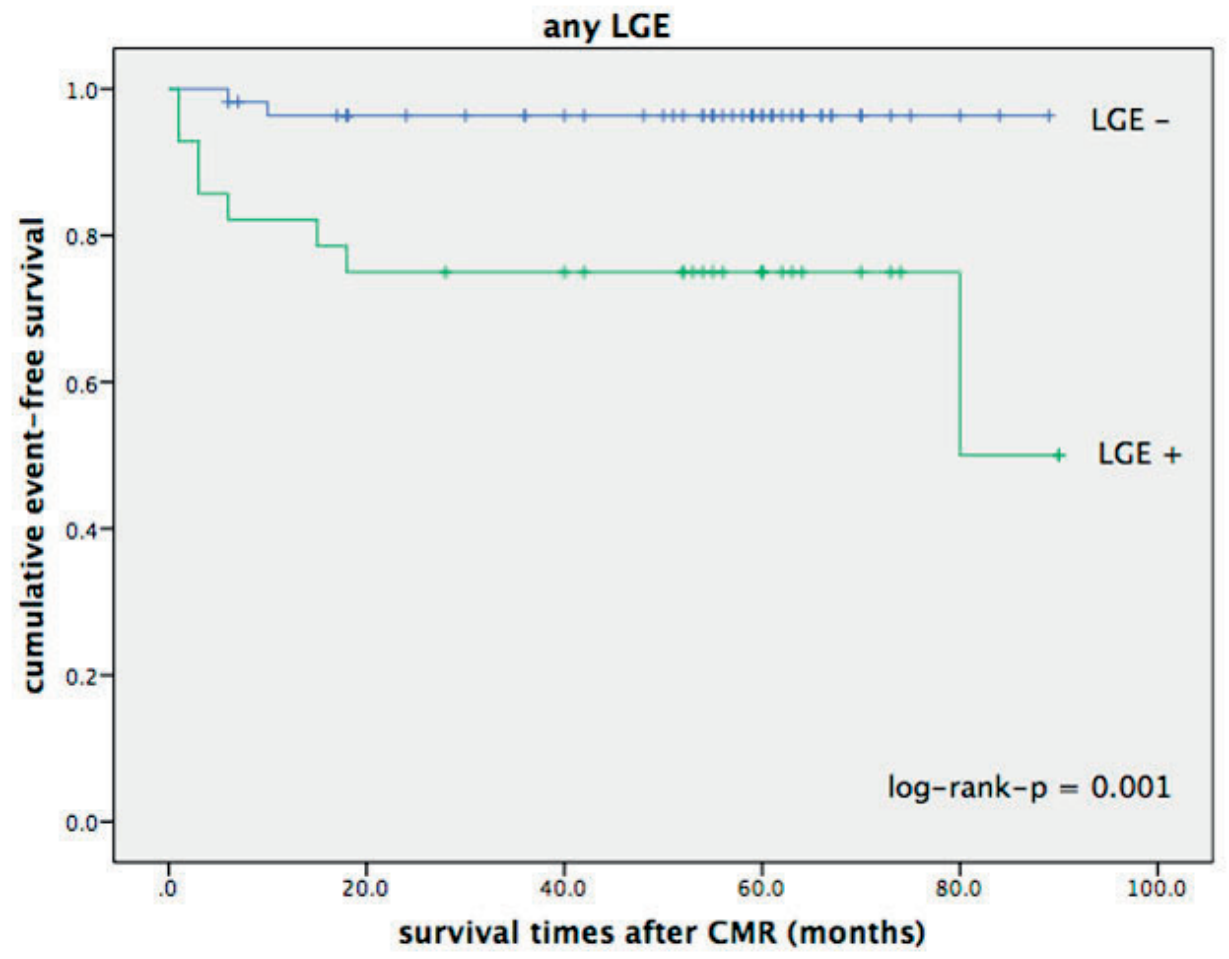

Patients at risk

\begin{tabular}{llllll}
\hline Months & 0 & 20 & 40 & 60 & 80 \\
\hline No LGE & 57 & 44 & 39 & 20 & 2 \\
Any LGE & 27 & 21 & 19 & 9 & 1 \\
\hline
\end{tabular}

Figure 2C: Kaplan-Meier survival curve for any myocardial LGE (composite primary end point). 


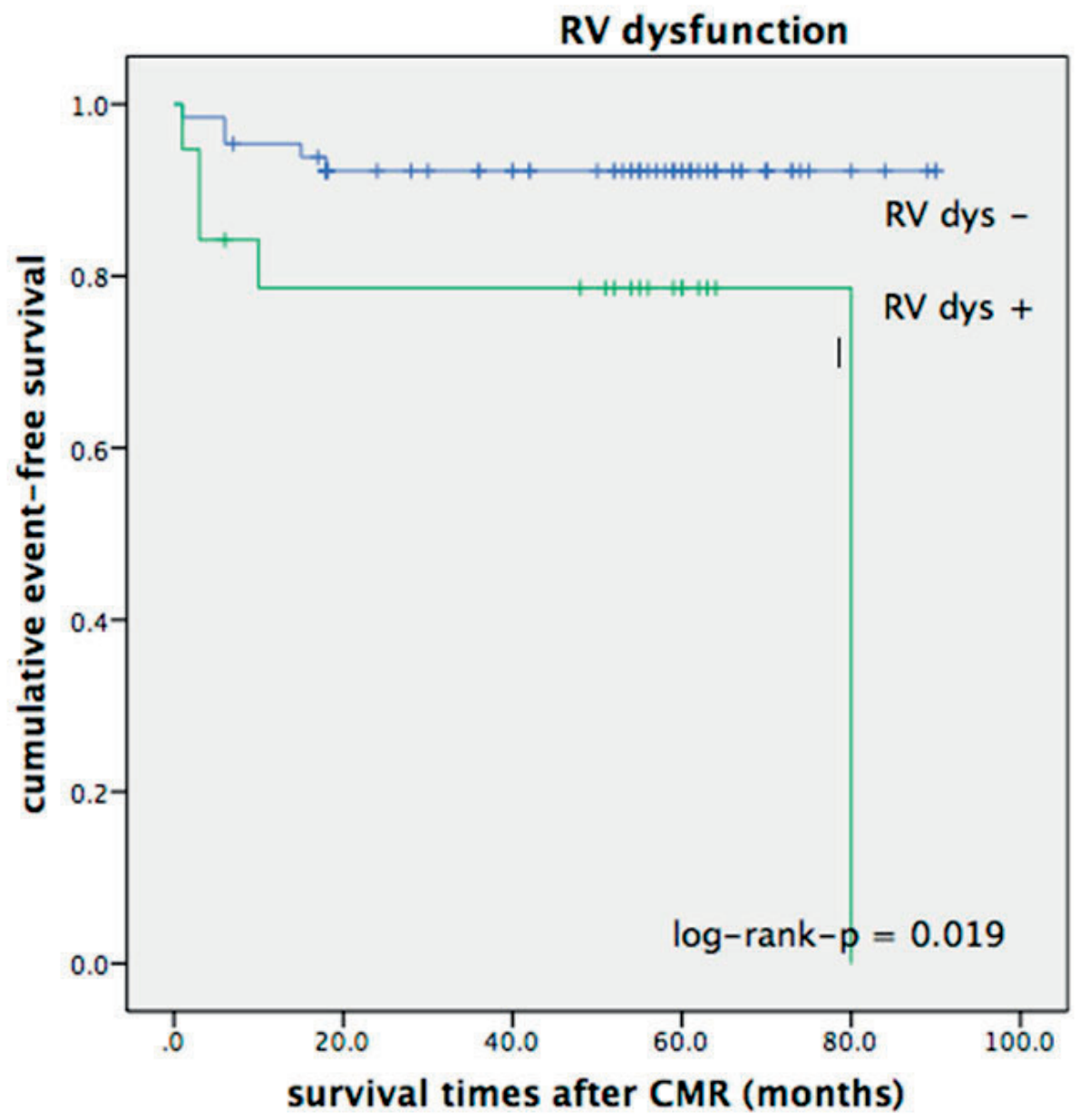

Patients at risk

\begin{tabular}{llllll}
\hline Months & 0 & 20 & 40 & 60 & 80 \\
\hline RV dysfunction - & 66 & 45 & 25 & 26 & 4 \\
RV dysfunction + & 18 & 14 & 14 & 6 & 0 \\
\hline
\end{tabular}

Figure 2D: Kaplan-Meier survival curve for RV dysfunction (composite primary end point). 


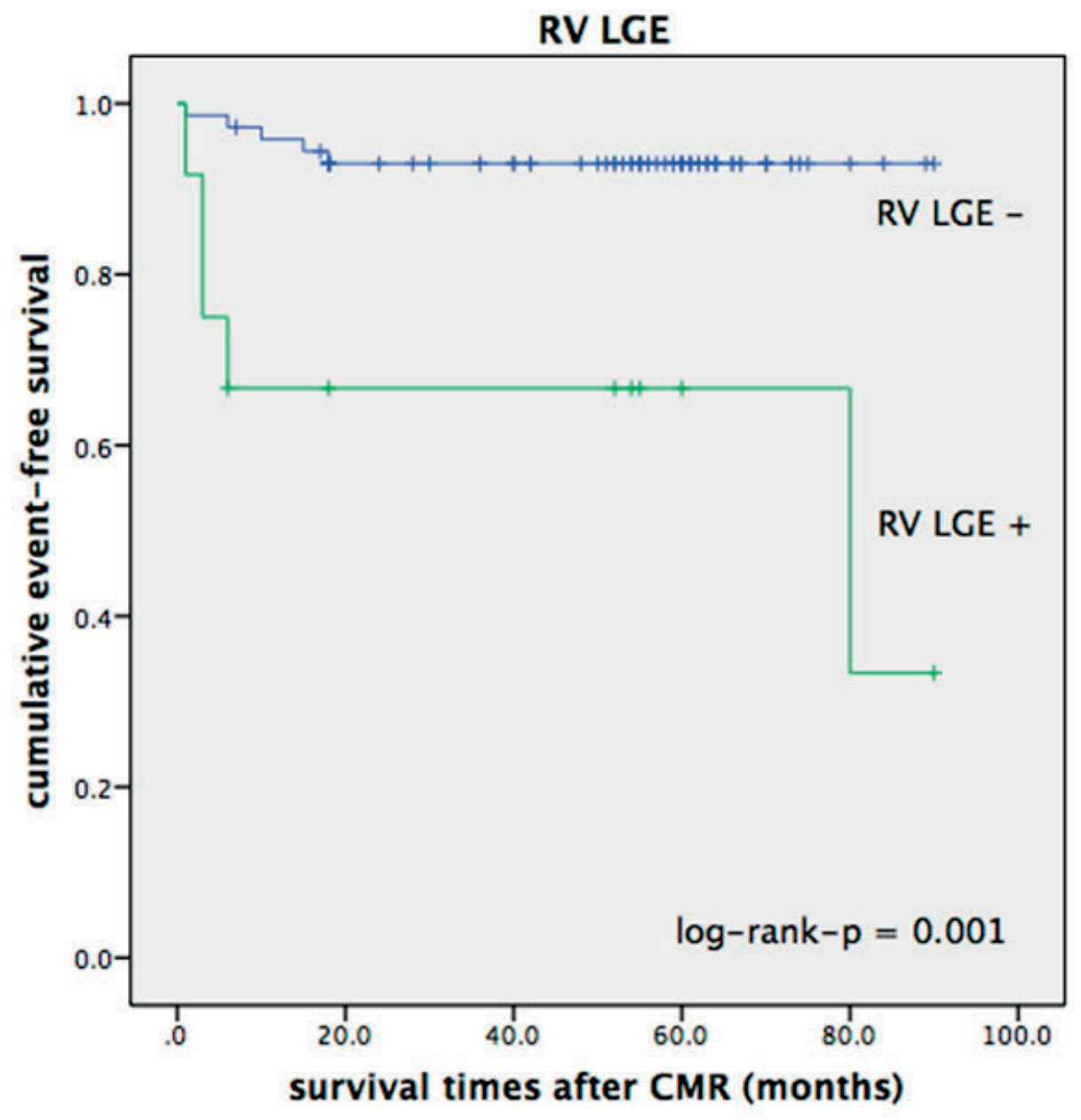

Patients at risk

\begin{tabular}{llrrrl}
\hline Months & 0 & 20 & 40 & 60 & 80 \\
\hline No LGE & 72 & 59 & 53 & 27 & 3 \\
RV LGE & 12 & 6 & 6 & 2 & 1 \\
\hline
\end{tabular}

Figure 2E: Kaplan-Meier survival curve for RV LGE (composite primary end point). 


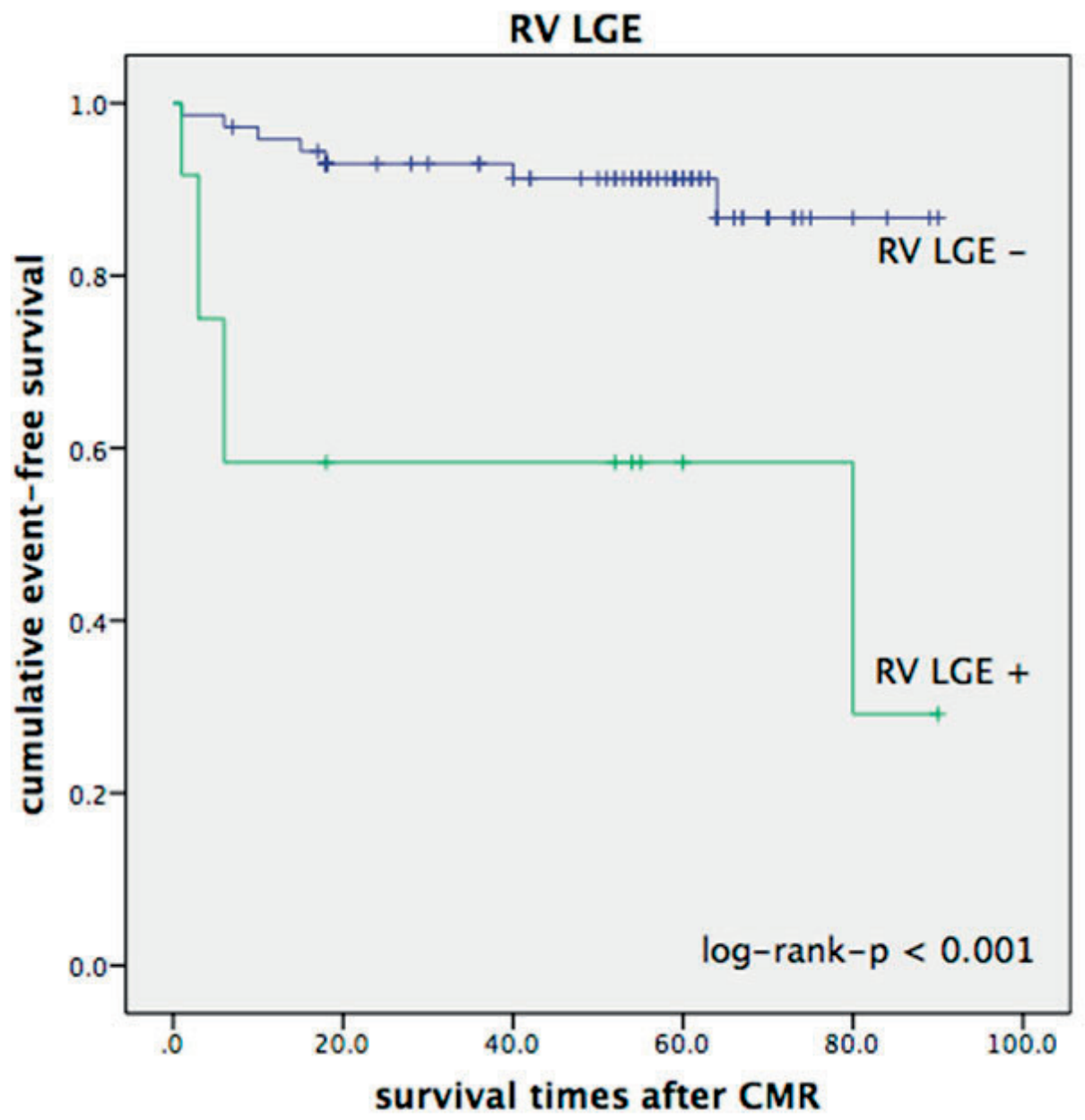

Patients at risk

\begin{tabular}{llrrrl}
\hline Months & 0 & 20 & 40 & 60 & 80 \\
\hline No LGE & 72 & 59 & 53 & 30 & 3 \\
RV LGE & 12 & 6 & 6 & 2 & 1 \\
\hline
\end{tabular}

Figure 2F: Kaplan-Meier survival curve for RV LGE (composite secondary end point). 


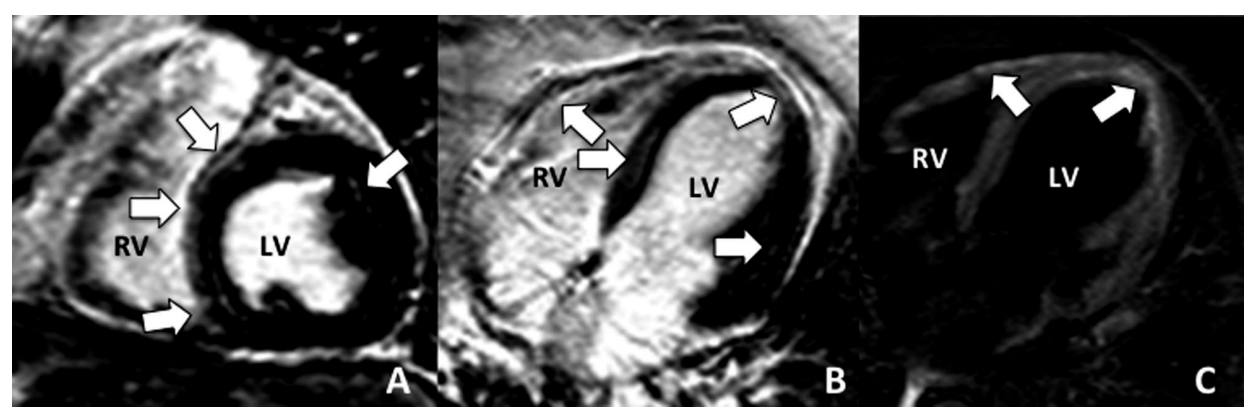

Figure 3.1: DECMR of a middle-aged female patient who presented in CCF with frequent nsVT, left bundle branch block, LVEF 38\% and extensive patchy LGE (38\% LV mass), predominantly involving the right-sided interventricular septum, RV free wall, and LV mid- and sub-epicardial layers (arrows). A biventricular PM/ICD was implanted. During follow up of 62 months no adverse events occurred. (A. Inversion Recovery-Gradient Echo sequence, horizontal long axis view, B. Inversion Recovery-Gradient Echo sequence, short axis view, C.T2 weighted Spin Echo sequence - increased signal signifies inflammation of the RV free wall and LV apex)

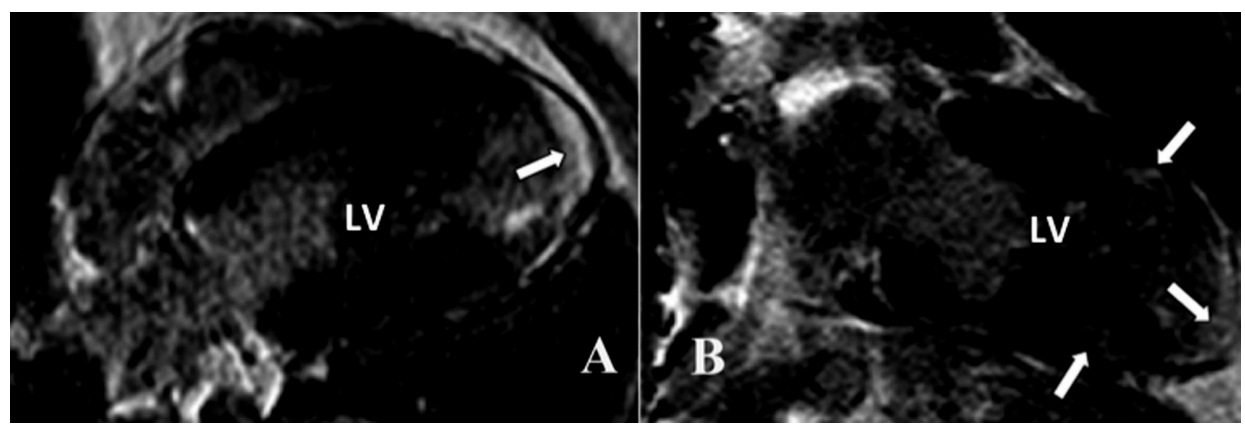

Figure 3.2 DECMR in a patient with preserved systolic LV function, who presented with dyspnea and palpitations demonstrating predominantly apical LGE (arrows) (LV LGE 8\%). Holter monitoring detected frequent episodes of nsVT. ${ }^{111}$ Indium-pentetreotide scintigraphy demonstrated active apical inflammation. An ICD was implanted and immune-suppressive and anti-arrhythmic therapy initiated. (A. Inversion Recovery-Gradient Echo sequence, horizontal long axis view, B. Inversion Recovery-Gradient Echo, vertical long axis view) 


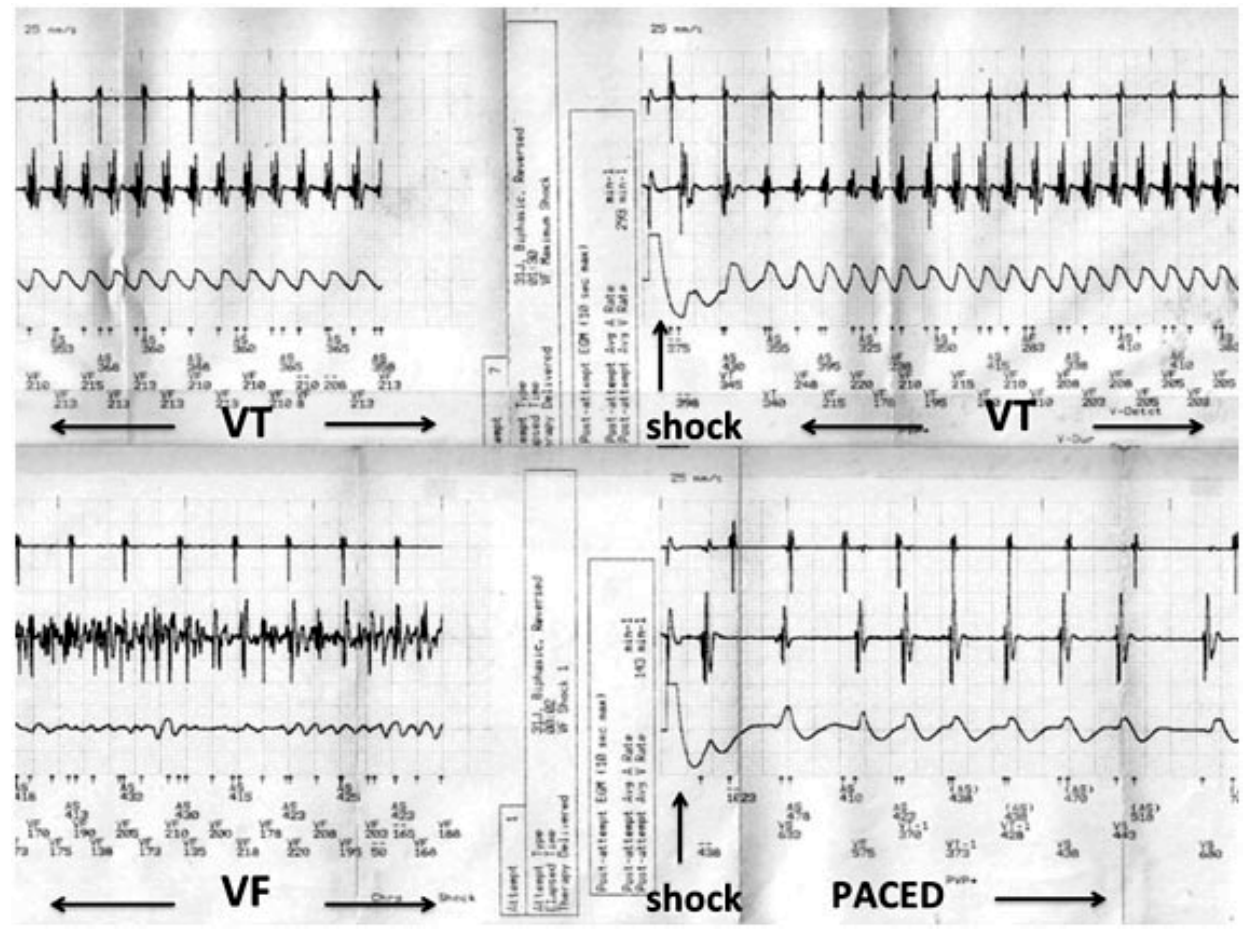

Figure 3.3 The implanted ICD detected episodes of sVT after 18 months. The top strip demonstrates unsuccessful ICD discharge for fast monomorphic VT, with eventual VF successfully reverted to a paced rhythm

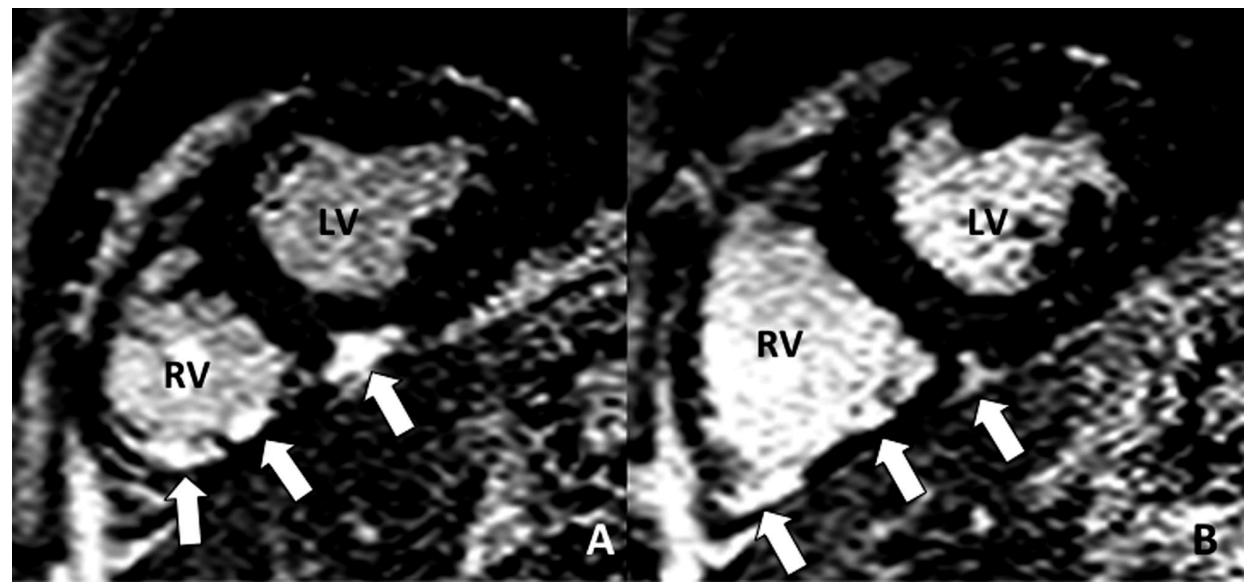

Figure 3.4 DECMR of a middle-aged male patient who presented with pre-syncopal symptoms due to sustained VT originating from the RV. DECMR demonstrated extensive patchy LV, in addition to LGE of the inferior RV segments and inferior RV insertion point (arrows). Anti-arrhythmic and immune suppressive treatment was started, and an ICD was implanted. PES on treatment was unable to illicit any monomorphic VT. During follow up of 79 months no adverse events occurred. (A. and B. Inversion Recovery-Gradient Echo sequence, short axis views 



\section{Chapter 9}

\section{General discussion}

Sarcoidosis is a multi-system granulomatous disorder of unknown etiology with symptomatic cardiac presentation in approximately five percent of patients. (1)

The clinical features of cardiac sarcoidosis (CS) include congestive heart failure, cor pulmonale, supraventricular- and ventricular arrhythmias, conduction disturbances, ventricular aneurysms, pericardial effusion and sudden death. (2)

The diagnosis of CS is made in the co-existence of non-caseating granulomas on myocardial biopsy or biopsies of any extra-cardiac tissue (with the exclusion of other causes for granulomatous inflammation such as mycobacterial or fungal infection), and cardiovascular abnormalities for which other possible causes have been excluded. $(2,3,4,5)$ The prevalence of sarcoidosis in The Netherlands is estimated to be 20-30 per 100000 population, while the prevalence of cardiac involvement was unknown until we published our findings in 2005. Postmortem studies in the United States suggested cardiac involvement in $20-30 \%$ percent of patients with sarcoidosis. $(6,7)$ In Japan cardiac involvement is reported to be present in as many as $58 \%$ of sarcoidosis patients, and is responsible for as many as $85 \%$ of deaths from sarcoidosis. (8,9) As recent as in 2003 the illustrious OP Sharma (1936-2012), professor of medicine at the Keck School of Medicine (UCLA, Los Angeles), and life-long scholar of sarcoidosis, stated in an editorial entitled "Diagnosis of cardiac sarcoidosis. An imperfect science, a hesitant art" (10) "The diagnosis should de established early and accurately, and the treatment should be aggressive and effective. Both of these conditions have remained only partially realized. The search for a safe, reliable, and easily available diagnostic test for CS continues. "In 1988 uncontrasted T1-weigthed Cardiovascular magnetic resonance (CMR) was first used to demonstrate high-intensity mass lesions in the basal interventricular septum and apico-lateral left ventricular (LV) segment of a 30-year-old Caucasian man with syncopal episodes. (11) Since that first case-report in 1988 the number of publications on CMR in CS has accelerated from 1 manuscript per annum to over one per week. (PubMed, retrieved January 2019, total number of published manuscripts 534) Over the last 30 years CMR has continued to evolve. CMR is able to delineate and quantify the amount as well as the distribution of the pathological substrate, it being myocyte necrosis, infiltration, deposition or focal or diffuse interstitial replacement fibrosis. CMR provides superior image quality, without exposing patients to ionizing radiation, whilst generating direct information of the tissue characteristics of the myocardium. When we set out to study the promise of delayed contrast-enhanced CMR (DECMR) in the diagnosis, management and risk assessment of CS, only few case-reports had been published. Clinicians relied on electrocardiography, Doppler echocardiography, and ${ }^{201}$ thallium SPECT, ${ }^{67}$ gallium 
SPECT, or technetium ${ }^{99} \mathrm{MIBI}$ SPECT. DECMR, positron emission tomography (PET), and hybrid PET/CMR have overtaken these diagnostic tools.

Chapter 3 of this thesis reported on the aim to determine the prevalence of cardiac involvement in patients with pulmonary sarcoidosis (PS) in The Netherlands.

In 2005 we retrospectively evaluated the prevalence of cardiac involvement in 101 consecutive patients with pulmonary sarcoidosis (PS) who had been evaluated at the University Medical Centers of Rotterdam and Maastricht between 1998-2004. Our study was the largest published series to include CMR and first to systematically evaluate cardiac involvement in patients with sarcoidosis in The Netherlands. Standard assessment included 12-lead electrocardiography (ECG), ambulatory ECG, Doppler echocardiography, ${ }^{201}$ thallium SPECT, DECMR, coronary angiography to exclude coronary artery disease when indicated. CS was diagnosed according to a modified guideline of the Japanese Ministry of Health and Welfare (1993). Because of the limited diagnostic yield and invasive nature of the procedure with an associated risk of morbidity, we felt not justified to systematically subject patients who were screened for CS to endo-myocardial biopsy (EMB), and only performed biopsies in 9 patients. For the purpose of the present study the Japanese guideline was modified by excluding EMB as a diagnostic parameter. Sixteen of the 19 (84\%) patients who presented with cardiac symptoms and 3 of the $82(4 \%)$ who were screened for cardiac involvement were diagnosed with CS. DECMR diagnosed abnormalities suggestive of CS in 24 of $87(28 \%)$ study patients. The prevalence of cardiac involvement in those screened for this condition based on the modified Guideline or DECMR were respectively $4 \%$ and $15 \%$. The prognosis was good. The patients who presented with cardiac failure or ventricular arrhythmias due to CS had significant morbidity and a mortality rate of $25 \%$ during a mean follow up of 15 months. Nine (47\%) patients had a pacemaker and/or ICD implanted. The prevalence of CS among Dutch patients with PS seems rather low. Nevertheless, looking at the grim prognosis of CS, physicians should consider regular screening of their PS patients for cardiac involvement. Relatively straight forward detection methods may be used for this purpose.

The introduction of the ICD might improve outcome in CS. During a mean follow up of 15 months (3-54 months), the ICD delivered appropriate therapy in only 1 of 6 patients.

Chapters 3, 4, 5, 6 and 7 reported on the aim of this thesis to determine the distribution and extent of myocardial sarcoidosis with DECMR. Our findings correlated with those at postmortem reported by Silverman and Roberts. $(6,7)$

DECMR demonstrated patchy 3 layer left ventricular (LV), mid-myocardial and epicardial enhancement in predominantly the basal interventricular septum (IVS), lateral LV, and the anterolateral papillary muscles in approximately $30 \%$ of PS patients. Resulting eccentric mitral regurgitation was significant in few patients. In several patients confluent, (near) transmural late gadolinium enhancement (LGE) was present, generally with local loss of wall thickness, signifying replacement fibrosis. Chapter 7 described the first prospective CMR study to specifically report on the prevalence, and distribution of RV involvement due to CS in 87 PS patients. LGE was demonstrated in 30 patients (34\%). Right ventricular LGE was present in $16 \%$ of patients diagnosed with pulmonary sarcoidosis, and in $48 \%$ of patients with LV enhancement. The pattern of RV LGE consisted predominantly in free 
wall enhancement (8 pts), ventricular insertion point enhancement (10 pts) and right-sided IVS enhancement (11 pts). The presence of RV LGE correlated with pulmonary arterial hypertension, RV systolic dysfunction, hypertrophy and dilation.

In Chapter 4 we determined that DECMR was of additional diagnostic value to standard assessment with electrocardiography, Doppler-echocardiography, ${ }^{201}$ thallium scintigraphy and DECMR in 55 PS patients who underwent evaluation for cardiac involvement between 2002-2004. DECMR provided accurate estimation of the extent of cardiac involvement and revealed signs of early infiltration not detected by standard assessment. Standard evaluation diagnosed CS in 13 while DECMR diagnosed myocardial scarring ( $2.5 \pm 1.9$ segments $)$ in an additional 6 patients. The extent of LGE correlated with disease duration, ventricular dimensions and function, severity of mitral regurgitation and the presence of ventricular tachycardias. Patients in whom cardiac involvement was only diagnosed with DECMR had significantly less myocardial scarring and functional impairment compared to patients diagnosed with standard assessment. Our study was the first to evaluate the value of comprehensive CMR assessment in addition to resting and ambulatory ECGs, Dopplerechocardiography and ${ }^{201}$ thallium scintigraphy. We found DECMR to be highly sensitive in detecting abnormalities that suggest cardiac involvement in patients with histologically confirmed PS. Our findings demonstrate a strong correlation between electrocardiographic and echocardiographic abnormalities and the amount of LV LGE with DECMR. The distribution of LGE correlated well with the localization of inflammation and fibrosis reported in postmortem studies of patients with CS. $(6,7)$ Limited, non-transmural or patchy myocardial scar tissue, frequently found at postmortem evaluation of CS patients, may remain undetected by electrocardiography, ultrasound or scintigraphy, but be detected by DECMR due to its high spatial resolution and inversion-recovery gradient echo sequences that increase signal intensity differences by almost $500 \%$. (12,13) The additional value of CMR to standard assessment was demonstrated in 6 patients $(11 \%)$ in whom LV involvement was only diagnosed by DECMR. Since disease in this group was of shorter duration in comparison to the group diagnosed with standard assessment, and myocardial involvement limited, the findings in these patients may well reflect early involvement.

In Chapter 5 we determined the diagnostic accuracy of DECMR for cardiac involvement in patients with PS compared to the, in 2005, gold-standard diagnostic Japanese Guidelines (1993). We demonstrated DECMR to be a valuable diagnostic tool to determine cardiac involvement in patients with sarcoidosis. We performed an analysis of the 12-lead ECGs, 24-hours ambulatory ECGs, echocardiograms, thallium scintigrams and DECMR studies in 58 biopsy proven PS patients assessed for CS. The diagnosis of CS was made in 12 of 58 (21\%) patients. The sensitivity and specificity of CMR were $100 \%$, and $78 \%$, the PPV and NPV $55 \%$ respectively $100 \%$, with an overall accuracy of $83 \%$. The low PPV of $55 \%$ reflects the ability of CMR to detect small myocardial lesions which had not resulted in conduction delay, repolarization abnormalities, or regional changes in wall thickness or function, and hence were not detected by ECG, echocardiography or SPECT. Long-term FU as discussed in Chapter $\mathbf{8}$ will determine the significance of these small asymptomatic contrast-enhancing LV lesions. 
In Chapter 6 we reviewed the value of DECMR in differentiating patients with CS from those with coronary artery disease (CAD) and recent myocardial infarcts. LGE by CMR is the most accurate non-invasive method to evaluate myocardial necrosis or focal replacement fibrosis caused by acute myocardial infarction, chronic myocardial infarction or NICM. (14) The distribution of LGE was valuable in differentiating between ischemic and non-ischemic myocardial scarring. (15-18) Since a significant number of patients with CS present with symptoms of heart failure or chest pain, similar in nature to those in patients with CAD, we aimed to determine whether CMR, and specifically the pattern of LGE, would allow us to distinguish the CS patients from patients with CAD and recent myocardial infarcts. Our study was the first to systematically compare the distribution of LGE in CS with the findings in patients with coronary artery disease. DECMR was helpful in the non-invasive differentiation between patients with CS and patients with CAD and previous myocardial infarcts. However, in approximately a third of patients with coronary artery disease, subepicardial or patchy, three layer LGE was found, a pattern that was suggestive of a non-ischemic etiology such as CS. Additional angiographic information was needed to correctly classify 11 patients with CAD and 3 with CS. Two experienced blinded observers classified patients by assessing the distribution of LGE. LV LGE was present in 29/30 CS (mean 3.8 segments, range $0-12$ ), all infarct (mean 4.3 segments, range $0-9$ ), and none of the patients in the control group. The amount of LV LGE did not differ significantly between CS and infarct patients $(19 \pm 11 \%$ and $19 \pm 12 \%, \mathrm{P}=0.8)$. The CS group exhibited a predominantly patchy, 3 layer LGE $(\mathrm{P}=0.01)$, whereas confluent transmural LGE $(\mathrm{P}=$ 0.04) with a vascular distribution $(\mathrm{P}<0.001)$ was prevalent in the infarct group. Significantly more RV LGE $(\mathrm{P}=0.01)$ and dilation $(\mathrm{P}=0.02)$ were found in the CS group. The two observers classified patients correctly as CS in $72 \%$ and $83 \%$ of cases, as ischemic in nature in $77 \%$ and $80 \%$ of cases, and as normal in $90 \%$ and $100 \%$ respectively.

In Chapter 7 we describe the prevalence and distribution of RV LGE in patients diagnosed with PS. This was the first prospective DECMR study to specifically evaluate the prevalence, and distribution of RV involvement in CS. Left ventricular involvement demonstrated by DECMR has been extensively reported. Until recently limited attention has been given to the prevalence, relevance and prognostic value of RV involvement in CS. CMR is the preferred imaging tool to evaluate the healthy and diseased RV. (19-23) We evaluated 87 patients diagnosed with PS with DECMR for RV involvement. Pulmonary artery pressures were non-invasively evaluated with Doppler echocardiography. Approximately $30 \%$ of an unselected patient population with PS had LV involvement, half of which had RV involvement. More extensive LV enhancement correlated with RV involvement. Right ventricular enhancement may result from direct infiltration and resulting scar, or pulmonary hypertension. Previous studies associated impaired systolic RV function and RV enhancement with ventricular tachy-arrhythmias. We demonstrated RV enhancement with DECMR to be mostly multi-focal, involve the septum and correlate with increased right ventricular volumes, hypertrophy and impaired systolic function. Pulmonary arterial hypertension correlated with the presence of RV LGE. The direct relationship between RV enhancement, its size and systolic function, as previously demonstrated in LV sarcoidosis, has not been reported before. Since RV sarcoidosis occurs in patients with more extensive LV disease, the reported prognostic relevance of RV disease may at least partly reflect the 
extent of left ventricular arrhythmogenic substrate. Right ventricular enhancement in sarcoidosis may be caused by direct granulomatous infiltration, but also be related to pulmonary hypertension. Right ventricular dilation, systolic impairment, and inflammation, as demonstrated with positron emission tomography (PET), in sarcoidosis have been associated with adverse outcome, particularly ventricular tachy-arrhythmias. (23-30) In Chapter 8 we investigated whether RV LGE carries similar prognostic relevance in the first prospective study of this kind. We determined that RV LGE predicts adverse events in addition to LV LGE during long-term follow up. Our study is the first to demonstrate the direct relationship between RV LGE, RV volumes, RV systolic impairment, sVT, and death of all cause. RV and LV LGE were demonstrated in respectively 12 (14\%) and 27 $(32 \%)$ of 84 consecutive biopsy proven PS patients. RV LGE, LV or biventricular LGE yielded Cox hazard ratios of 8.71 (95\% CI 1.90-23.81), 9.22 (95\% CI 1.96-43.45), and 12.09 (95\% CI 3.43-42.68) for the composite primary end-point of adverse cardiac outcomes. In a multi-variate model the predictive value of biventricular LGE for the composite primary and secondary end-points was strongest. Kaplan Meier event-free survival curves were most significant for RV LGE and biventricular LGE. Biventricular LGE at presentation is the strongest, independent predictor of adverse outcome during long-term follow up. Asymptomatic myocardial scar $<8 \%$ of LV mass carried a favorable long-term outcome. Right ventricular LGE indeed predicted ventricular tachy-arrhythmias and all cause death during follow up. In the absence of data on VT morphology it remains unclear whether the prognostic relevance of RV LGE is related to more extensive biventricular arrhythmogenic substrate or whether RV LGE itself is particularly arrhythmogenic. None of our patients with asymptomatic LGE suffered adverse cardiac events, while the majority of arrhythmic end-points $(75 \%)$ occurred in patients with LVEF $>35 \%$. Our findings are supported by the results of recent studies which evaluated the risk of SCD in CS patients, and also recorded appropriate ICD therapy in a large proportion of patients with LVEF $>35 \%$. $(3,20,23,25)$ The risk of VT and SCD in CS seems predominantly related to amount and distribution of granulomatous infiltration and scar, and not primarily the systolic function. Arrhythmic substrate imaging with DECMR and/or PET determines the risk of arrhythmic events more accurately than LVEF. $(3,20,23,25,20,28,30,31)$ The negative predictive value of LGE negative DECMR is excellent, with only one patient with LV LGE $<8 \%$ developing an arrhythmic event after 10 months of follow up. In the absence of prospective data, it seems prudent to follow asymptomatic sarcoidosis patients up with ECG and cardiac ultrasound, and evaluate suspected or confirmed CS with PET and/or DECMR. (3) Our studies support the recommendations of the 2014 HRS guidelines concerning the use of DECMR and device implantation. (3)

Until recently the prognosis of CS was considered dismal. Flemming et al reported $44 \%$ of 250 CS patients surviving 5 years. (32) Roberts et al estimated survival after the development of cardiac symptoms to be 2 years. (6) Long-term outcomes in patients with CS have remarkably improved due to accurate diagnostic imaging with CMR and PET, modern medical heart failure management, including device therapy, arrhythmia ablation and transplantation in selected patients. (33-38) Current annual mortality rates range from 0 4.2/100 pts compared to 7.5-12/100 pts as previously reported by Yazaki (2001) and Fleming (1986). (39,40) Five-year survival rates have improved from 44\% to current 5 and 10 - 
year survival rates of over $90 \%$. (32-40) Our studies are limited by the relatively small number of predominantly Caucasian, patients and few events. African-American and Japanese populations generally have higher rates of cardiac involvement with more extensive myocardial involvement, and possibly a more malignant course. $(2,8,9)$ LGE is the strongest, independent CMR predictor of future adverse cardiac events in sarcoidosis patients. RV involvement in addition to LV LGE increases risk of adverse cardiac outcomes and death of all causes. DECMR should ideally be performed in sarcoidosis patients with cardiac symptoms. Asymptomatic patients with LGE $<8 \%$ of LV mass and mildly impaired LVEF may not benefit from device therapy, and be monitored. When we first evaluated the diagnostic accuracy of DECMR the only published Guidelines had not included this modality yet. (41) Since 2007 DECMR has gradually been included in the Diagnostic and Management Guidelines of 5 professional Societies, and the prominence of DECMR has increased from being a minor to becoming a major criterium for the diagnosis of CS with additional prognostic relevance. $(3,42-47)$ Recent editorials have captured the current status of CMR in the management of (suspected) CS. "CMR has high diagnostic accuracy and prognostic value for the evaluation of individuals with suspected CS.” (48) “... novel CMR mapping techniques can detect subclinical myocardial changes in sarcoidosis, adding value to the diagnosis of patients otherwise being classified as having normal cardiac findings." (49) The limits of CMR have not been reached. Ultra-high field CMR and compressedsensing techniques will continue to shorten scan times, improve image resolution and quality, which will allow for detailed assessment of the atria and RV. (50,51,52,53) Prospective studies will help determine the value of native T1 mapping, and the assessment of the extra cellular myocardial volume (ECV) by the use of native and post-contrast T1 maps. Hybrid PET-CMR imaging with T2 mapping have optimized detection and management of active inflammation. Mapping multiple relaxation times simultaneously will similarly save time and remove confounders introduced by the interaction of relaxation times. Threedimensional mapping allows for more complete characterization of disease distribution, socalled CMR fingerprinting of the myocardium, which implies the generation of structural, mechanical, functional as well as biochemical information. $(\mathbf{5 0 , 5 4 , 5 5 )}$ DECMR will help plan arrhythmia ablation and potentially increase success rates and outcomes. Recent advances have made real-time CMR guided EMB in patients with suspected isolated CS and CMR-guided ablations of (supra)ventricular arrhythmias a reality. $(\mathbf{5 6 , 5 7 , 5 8 )}$ 


\section{References}

1. Valeyre D, Prasse A, Nunes H, Uzunhan Y, Brillet PY, Müller-Quernheim J. Sarcoidosis. Lancet 2014;383:1155-1167. doi: 10.1016/S0140-6736(13)60680-7.

2. Birnie DH, Nery PB, Ha AC, Beanlands RS. Cardiac sarcoidosis. J Am Coll Cardiol. 2016;26;68(4):411-21. doi: 10.1016/j.jacc.2016.03.605.

3. Birnie DH, Sauer WH, Bogun F, Cooper JM, Culver DA, Duvernoy CS, Judson MA, Kron J, Mehta D, Cosedis Nielsen J, Patel AR, Ohe T, Raatikainen P, Soejima K. HRS Expert consensus statement on the diagnosis and management of arrhythmias associated with cardiac sarcoidosis. Heart Rhythm 2014;11(7):13051323. doi: 10.1016/j.hrthm.2014.03.043.

4. Ikeda U. Editorial. Diagnosis of cardiac sarcoidosis - What is the role of endo-myocardial biopsy? J Cardiol Cases 2015;12(3):72-73. doi:10.1016/j.jccase.2015.01.015.

5. Vita T, Okada DR, Veillet-Chowdhury M, Bravo PE, Mullins E, Hulten E, Agrawal M, Madan R, Taqeti VR, Steigner M, Skali H, Kwong RY, Stewart GC, Dorbala S, Di Carli MF, Blankstein R. Complementary value of cardiac magenetic resonance imaging and positron emission tomography/computed tomography in the assessment of cardiac sarcoidosis. Circ Cardiovasc Imaging 2018;11:e007030. doi: 10.1161/ circimaging.117.007030.

6. Roberts WC, McAllister HA Jr, Ferrans VJ. Sarcoidosis of the heart: a clinico-pathological study of 35 necropsy patients (group 1) and review of 78 previously described necropsy patients (group II). Am J Med 1977;63:86-108.

7. Silverman KJ, Hutchins GM, Bulkley BH. Cardiac sarcoid: a clinico-pathologic study of 84 unselected patients with systemic sarcoidosis. Circulation 1979;58:1204-1211.

8. Matsui Y, Iwai K, Tachibana T, Fruie T, Shigematsu N, Izumi T, Homma AH, Mikami R, Hongo O, Hiraga Y, Yamamoto M. Clinicopathological study on fatal myocardial sarcoidosis. Ann NY Acad Sci 1976;278:45569.

9. Tachibana T, Iwai K, Takemura T. Study on the cause of death in patients with sarcoidosis in Japan. XII World Congress on Sarcoidosis, Kyoto, Japan, September 8-13,1991. Abstract

10. Sharma OP. Diagnosis of cardiac sarcoidosis. An imperfect science, a hesitant art" Chest 2003;123(1):18-19. doi: $10.1378 /$ chest.123.118.

11. Riedy K, Fisher MR, Belic N, Koenigsberg DI.MR Imaging of myocardial sarcoidosis. Am J Roentgenol 1988;151:915-916.

12. Wagner A, Mahrholdt H, Holly TA, Elliott MD, Regenfus M, Parker M, Klocke FJ, Bonow RO, Kim RJ, Judd RM. Contrast-enhanced MRI and single photon emission computed tomography (SPECT) perfusion imaging for the detection of subendocardial myocardial infarcts: an imaging study. Lancet 2003;361:374-379.

13. Simonetti OP, Kim RJ, Fieno DS, Hillenbrand HB, Wu E, Bundy JM, Finn JP, Judd RM. An improved MR imaging technique for the visualzation of myocardial infarction. Radiology 2001;218:215-223.

14. Pennell DJ, Sechtem UP, Higgins CB, Manning WJ, Pohost GM, Rademakers FE, van Rossum AC, Shaw LJ, Yucel EK. Special article. Clinical indications for cardiovascular magnetic resonance (CMR): Consensus Panel report. European Heart Journal 2004;25:1940-1965. doi:

15. Hunold P, Schlosser T, Vogt FM, Eggebrecht H, Schmermund A, Bruder O, Schuler WO, Barkhausen J. Myocardial late enhancement in contrast-enhanced cardiac MRI: distinction between infarction scar and non-infarction-related disease. Am J Roentgenol 2005;184(5):1420-6.

16. Lim RP, Srichai MB, Lee VS. Non-Ischemic Causes of delayed myocardial hyper-enhancement on MRI. Am J Roentgenol 2007; 188:1675-1681. Doi:

17. Mahrholdt H, Wagner A, Judd RM, Sechtem U, Kim RJ. Delayed enhancement cardiovascular magnetic resonance assessment of non-ischemic cardiomyopathies. Eur Heart J 2005;26:1461-1474.

18. Laissy JP, Hyafil F, Feldman LJ, Juliard JM, Schouman-Claeys E, Steg PG, Faraggi M. Differentiating acute myocardial infarction from myocarditis: diagnostic value of early- and delayed-perfusion cardiac MR imaging. Radiology 2005;237:75-82.

19. Samar HY, Thompson DV, Doyle M, Williams RB, Yamrozik JA, Reddy ST, Shah M, Biederman RW. arcoidosis; is it confined to just the LV? An RV LGE study. From 17th Annual SCMR Scientific Sessions, New Orleans, LA, USA. 16-19 January 2014.

20. Patel MR, Cawley PJ, Heitner JF, Klem I, Parker MA, Jaroudi WA, Meine TJ, White JB, Elliott MD, Kim HW, Judd RM, Kim RJ. Detection of myocardial damage in patients with sarcoidosis. Circulation 2009;120:1969-77. doi: 10.1161/CIRCULAIONAHA.109.851352. 
21. Cheong BY, Muthupillai R, Nemeth M, Lambert B, Dees D, Huber S, Castriotta R, Flamm SD. The utility of delayed-enhancement magnetic resonance imaging for identifying non-ischemic myocardial fibrosis in asymptomatic patients with biopsy-proven systemic sarcoidosis. Sarcoidosis Vasc Diff Lung Dis 2009;26:3946.

22. Nadel J, Lancefield T, Voskoboinik A, Taylor AJ. Late gadolinium enhancement identified with cardiac magnetic resonance imaging in sarcoidosis patients is associated with long-term ventricular arrhythmia and sudden cardiac death. Eur Heart J Cardiovasc Imaging 2015;16:1634-41. doi: 10.1093/ehjci/jeu294.

23. Crawford T, Mueller G, Sarsam S, Prasitdumrong H, Chaiyen N, Gu X, Schuller J, Kron J, Nour KA, Cheng A, Ji SY, Feinstein S, Gupta S, Ilg K, Sinno M, Abu-Hashish, Al-Mallah M, Sauer WH, Ellenbogen K, Morady F, Bogun F. Magnetic resonance imaging for identifying patients with cardiac sarcoidosis and preserved or mildly reduced left ventricular function at risk of ventricular arrhythmias. Circ Arrhythm Electrophysiol 2014;7:1109-15. doi: 10.1161?CIRCEP.113.000156.

24. Gulati A, Ismail TF, Jabbour A, Alpendurada F, Guha K, Ismail NA, Raza S, Khwaja J, Brown TD, Morarji K, Liodakis E, Roughton M, Wage R, Pakrashi TC, Sharma R, Carpenter JP, Cook SA, Cowie MR, Assomull RG, Pennell DJ, Prasad SK. The prevalence and prognostic significance of right ventricular systolic dysfunction in non-ischemic dilated cardiomyopathy. Circulation 2013;128(15):1623-36. doi: 10.1161/CIRCULATIONAHA.113.002518.

25. Murtagh G, Laffin LJ, Beshai JF, Maffessanti F, Bonham CA, Patel AV, Yu Z, Addetia K, Mor-Avi V, Moss JD, Hogarth DK, Sweiss NJ, Lang RM, Patel AR. Prognosis in myocardial damage in sarcoidosis patients with preserved left ventricular ejection fraction. Risk stratification using cardiac magnetic resonance. Circ Cardiovasc Imag 2016;9:e003738. doi: 10.1161/CIRCIMAGING.115.003738.

26. Schuller JL, Zipse M, Crawford T, Bogun F, Beshai J, Patel AR, Sweiss NJ, Nguyen DT, Aleong RG, Varosy PD, Weinberger HD, Sauer WH. Implantable cardioverter defibrillator therapy in patients with cardiac sarcoidosis. J Cardiovasc Electrophysiol 2012;23:925-9. doi: 10.1111/j.1540-8167.2012.02350.x.

27. Patel AR, Klein MR, Chandra S, Spencer KT, DeCara JM, Lang RM, Burke MC, Garrity ER, Hogarth DK, Archer SL, Sweiss NJ, Beshai JF. Myocardial damage in patients with sarcoidosis and preserved left ventricular systolic function: an observational study. Eur J Heart Fail 2011;13:1231-37. doi: 10.1093/eurjhf/hfr099.

28. Muser D, Santangeli P, Patahk RK, Castro SA, Liang JJ, Magnani S, Hayashi T, Garcia FC, Hutchinson MD, Supple GE, Frankel DS, Riley MP, Lin D, Schaller RD, Desjardins B, Dixit S, Callans DJ, Zado ES, Marchlinski FE. Long-term outcomes of catheter ablation of ventricular tachycardia in patients with cardiac sarcoidosis. Circ Arrhythm Electr 2016;9(8):e004333. doi: 10.1161/CIRCEP.116.004333.

29. Ekström K, Lehtonen J, Hänninen H, Kandolin R, Kivistö S, Kupari M. Magnetic resonance imaging as a predictor of survival free of life-threatening arrhythmias and transplantation in cardiac sarcoidosis. J Am Heart Assoc 2016;5:e003040. doi: 10.1161/JAHA.115.003040.

30. Blankstein R, Osborn M, Naya M, Waller A, Kim CK, Murthy VL, Kazemian P, Kwong RY, Tokuda M, Skali H, Padera R, Hainer J, Stevenson WG, Dorbala S, Di Carli MF. Positron emission tomography enhances prognostic assessments of patients with suspected cardiac sarcoidosis. J Am Coll Cardiol 2014:63:329-36. doi: 10.1016/j.jacc.2013.09.022.

31. Jefic D, Joel B, Good E, Morady F, Rosman H, Knight B, Bogun F. Role of radiofrequency catheter ablation of ventricular tachycardia in cardiac sarcoidosis: Report from a multi-centre registry. Heart Rhythm 2009;6:189-195. doi: 10.1016/j.hrthm.2008.10.039.

32. Flemming H, Baily S. Cardiac sarcoidosis. In: James DG, ed. Sarcoidosis and other granulomatous disorders. (volume 73) New York, NY: Marcel Dekker, 1994;323-334.

33. Kron J, Sauer W, Mueller G, Schuller J, Bogun F, Sarsam S, Rosenfeld L, Mitiku TY, Cooper JM, Mehta D, Greenspon AJ, Ortman M, Delurgio DB, Valadri R, Narasimhan C, Swapna N, Singh JP, Danik S, Markowitz SM, Almquist AK, Krahn AD, Wolfe LG, Feinstein S, Ellenbogen KA, Crawford T. Outcomes of patients with definite and suspected isolated cardiac sarcoidosis treated with an implantable cardiac defibrillator. J Interv Card Electrophysiol 2015;43(1):55-64. doi: 10.1007/s108040-015-9978-3.

34. Chapelon-Abric C, Sene D, Saadoun D, Cluzel P, Vignaux O, Costedoat-Chalumeau N, Piette JC, Cacoub P. Cardiac sarcoidosis: Diagnosis, therapeutic management, and prognostic management. Arch Cardiovasc Dis 2017;110(8-9):456-465. Pii: S1875-2136(17)30096-7. doi: 10.1016/j.acvd.2016.12.014

35. Zhou Y, Lower EE, Li H, Costae A, Attari M, Baughman RP. Cardiac sarcoidosis: the impact of age and implanted devices on survival. Chest 2017;151(1):139-148. doi: 10.1016/j.chest.2016.08.1457 
36. Fussner LA, Karlstedt E, Hodge DO, Fine NM, Kalra S, Carmona EM, Utz JP, Isaac DL, Cooper LT. Management and outcomes of cardiac sarcoidosis: a 20-year experience in two tertiary care centres. Eur J Heart Fail 2018 Oct 31. doi: 10.1002/ejhf.1319 [Epub ahead of print]

38. Ghanizada M, Rossing K, Bundgaard H, Gustafsson F. Clinical presentation, management and prognosis of patients with cardiac sarcoidosis. Dan Med J 2018;65(4). Pii: A5462.

38. Smedema JP, van Geuns RJ, Ector J, Ainslie G, Heidbuchel H, Crijns HJGM. Right ventricular involvement and the extent of left ventricular enhancement with magnetic resonance predict adverse outcome in pulmonary sarcoidosis. ESC Heart Failure 2018; (1):157-171. doi: 10.1002/ehf2.12201.

39. Yazaki Y, Isobe M, Hiroe M, Morimoto A, Hiramitsu, Nakano T, Izumi T, Sekiguchi M. Prognostic determinants of long-term survival in Japanese patients with cardiac sarcoidosis treated with prednisone. Am J Cardiol 2001;88:1006-1010.

40. Fleming HA, Bailey SM. The prognosis of sarcoid heart disease in the United Kingdom. Ann N Y Acad Sci 1986;465:543-550. doi: 10.1111/j.1749-6632.

41. Hiraga H, Yuwai K, Hiroe M. Guideline for the diagnosis of cardiac sarcoidosis: study report on diffuse pulmonary diseases [in Japanese]. Tokyo, Japan: The Japanese Ministry of Health and Welfare; 1993;23-24.

42. Revised Guidelines for Diagnosis Cardiac Sarcoidosis 2006 (Japanese Society of Sarcoidosis and Other Granulomatous Disorders) criteria Japanese Ministery of Health 2006. Japanese Ministery Health Welfare. Diagnostic standard and guidelines for sarcoidosis. Jpn J Sarcoidosis Granulomatous Disord (Jpn) 2007;27:89-102.

43. Ishida Y, Yoshinaga K, Miyagawa M, Moroi M, Kondoh C, Kiso K, Kumita S. Recommendations for 18Ffluodeoxyglucose positron emission tomography imaging for cardiac sarcoidosis: Japanese Society of Nuclear Cardiology Recommendations. Ann Nucl Med 2014;28:393-403. doi: 10.1007/s12149-014-0806-0.

44. Costabel U, Skowasch D, Pabst S, Störk S, Schöpe C, Allewelt M, Worth H, Müller-Quernheim J, Grohé C. Diagnostik and Therapie der kardialen Sarkoidose. Pneumatologie 2014;68:124-132. doi: 10.1055/s-00331359197.

45. A joint procedural position statement on imaging in cardiac sarcoidosis: from the Cardiovascular and Inflammation \& Infection Committees of the European Association of Nuclear Medicine, the European Association of Cardiovascular Imaging, and the American Society of Nuclear Cardiology. Eur Heart J CVImaging 2017;18:1073-1089. doi:10.1093/ehjcvi/jex146

46. Terasaki F, Yoshinaga K. New guidelines for diagnosis of cardiac sarcoidosis in Japan. Ann Nucl Cardiol 2017;3:42-45. doi: 10.17996/anc.17-00042.

47. Slart RHJA, Glaudemans AWJM, Lancellotti P, Hyafil F, Blankstein R, Schwartz RG, Jaber WA, Russell R, Gimelli A, Rouzet F, Hacker, Gheysens O, Plein S, Miller EJ, Dorbala S, Donal E. A joint procedural position statement on imaging in cardiac sarcoidosis: From the Cardiovascular and Inflammation \& Infection Committees of the European Association of Nuclear Medicine, the European Association of Cardiovascular Imaging, and the American Society of Nuclear Cardiology. J Nucl Cardiol 2018;25(1):298-319. doi: 10.1007/s12350-017-1043-4.

48. Bravo PE, Taqueti VR. Cardiac MRI vs PET for the evaluation of cardiac sarcoidosis: Consider MRI first. JACC 2017; https://www.acc.or/latest-in-cardiology/articles/2017/ 04/10/08/43/cardiac-mri-vs-pet.

49. Ferreira VM, Piechnik SK. Seeing beyond the obvious. Subclinical cardiac sarcoidosis revealed by Cardiovascular Magnetic Resonance Mapping. Circ Cardiovasc Imag 2016;9:e005592. doi: 10.1161/CIRCIMAGING.116.005592.

50. Messroghli DR, Moon JC, Ferreira V, Grosse-Wortmann L, He T, Kellman P, Mascherbauer J, Nezafat R, Salerno M, Schelbert EB, Taylor AJ, Thompson R, Ugander M, van Heesweek RB, Friedrich MG. Clinical recommendations for cardiovascular magnetic resonance mapping of T1, T2, T2* and extracellular volume: A consensus statement by the Society for Cardiovascular Magnetic Resonance (SCMR) endorsed by the European Association for Cardiovascular Imaging (EACVI). J Cardiovascular Magn Res 2017;19:75-99. doi: 10.1186/s12968-017-0389-8.

51. Bhaskaran A, Kumar S, Kizana E, Thomas SP, Chik WWB. Multimodality imaging, electrophysiologic, electroanatomic, and histopathologic characterization of atrial sarcoidosis presenting with sinus arrest and reentrant right atrial flutter. HeartRhythm Case Rep 2018 Aug 16;4(10):469-474. doi: 10.1016/j.hrcr.2018.06.014

52. Spence S, Pena E, Thornhill RE, Nery PB, Birnie DH. Bi-atrial fibrosis detected using three-dimensional late gadolinium enhancement magnetic resonance imaging in a patient with cardiac sarcoidosis. Oxf Med Case Reports 2018 May 16;2018(5):omy016. doi:10.1093/omcr/omy016. eCollection 2018 May. 


\section{Chapter 9}

53. Kowallick JT, Kutty S, Edelmann F, Chiribiri A, Villa A, Steinmetz M, Sohns JM, Staab W, Bettencourt N, Unterberg-Buchwald C, Hasenfuß G, Lotz J, Schuster A. Quantification of left atrial strain and strain rate using Cardiovascular Magnetic Resonance myocardial feature tracking: a feasibility study. J Cardiovasc Magn Res 2014, 16:60 doi:10.1186/s12968-014-0060-6

54. Haaf P, Garg P, Messroghli DR, Broadbent DA, Greenwood JP, Plein S. Cardiac T1 Mapping and Extracellular Volume (ECV) in clinical practice: a comprehensive review. J Cardiovasc Magn Res 2016;18:89. doi: 10.1186/s12968-016-0308-4.

55. Hamilton JI, Jiang Y, Chen Y, Ma D, Lo W-C, Griswold M, Seiberlich N. MR fingerprinting for rapid quantification of myocardial T1, T2, and proton spin density. Mag Reson Med 2017;77(4):1446-1458. doi: $10.1002 / \mathrm{mrm} .26212$.

56. Unterberg-Buchwald C, Ritter CO, Reupke V, Wilke RN, Stadelmann C, Steinmetz M, Schuster A, Hasenfuß G, Lotz J, Eucker M. Targeted endomyocardial biopsy guided by real-time cardiovascular magnetic resonance. J Cardiovasc Magn Reson 2017 Apr 19;19(1):45. doi: 10.1186/s12968-017-0357-3.

57. Chubb H, Williams SE, Whitaker J, Harrison JL, Razavi R, O’Neill M. Cardiac electrophysiology under MRI guidance: an emerging technology. Arrhythm Electrophysiol Rev 2017;6(2):85-93. doi: 10.15420/aer.2017.1.2.

58. Mukherjee RK, Whitaker J, Williams SE, Razavi R, O’Neill MD. Magnetic resonance imaging guidance for the optimization of ventricular tachycardia ablation. Europace 2018;20(11):1721-1732. doi: 10.1093/europace/euy040 


\section{Chapter 10}

\section{Summary}

When we set out to formally evaluate the value of contrast-enhanced cardiovascular magnetic resonance (CMR) for the diagnosis and management of cardiac sarcoidosis (CS) little was known of the prevalence of cardiac sarcoidosis in The Netherlands.

In Chapter 2 we reviewed contrast-enhanced CMR in the diagnosis and management of CS. The aim of this review was to provide relevant clinical and pathophysiological background on CS, whilst introducing cardiovascular magnetic resonance imaging (CMR) as a technology, and detailing its past, current and future role in the management of CS. Chapter 3 details the prevalence of cardiac involvement in patients with pulmonary sarcoidosis (PS) assessed at two University Medical Centres in The Netherlands. We reviewed the findings in 101 consecutive patients who either presented to us with symptoms of cardiac involvement, or were screened for CS during 1998 -2004. Sixteen of the 19 (84\%) patients who presented with cardiac symptoms and 3 of the $82(4 \%)$ who were screened for cardiac involvement were diagnosed with CS (adapted Japanese 1993 criteria). Once PS patients developed CS, their prognosis became grim, with 4 deaths $(20 \%)$, and 9 cardiac devices implants (47\%). The CS patients who presented with cardiac failure or ventricular arrhythmias had significant morbidity and a mortality rate of $25 \%$ during a mean follow up of 15 months. In contrast, PS patients diagnosed with asymptomatic, small, focal scar fared well. Our study was the first to systematically evaluate cardiac involvement in patients with sarcoidosis in The Netherlands, and at that stage the largest published to employ CMR. Chapter 4 reported the additional value of gadolinium-enhanced CMR to standard assessment with electrocardiography, Doppler-echocardiography, and ${ }^{201}$ thallium scintigraphy for cardiac involvement in 55 patients with PS.

Of the 55 patients evaluated, standard evaluation diagnosed cardiac involvement in 13 while CMR diagnosed myocardial scarring in an additional $6(11 \%)$ patients. The extent of delayed enhancement correlated with disease duration, ventricular dimensions and function, severity of mitral regurgitation and the presence of ventricular tachycardias. Patients in whom cardiac involvement was only diagnosed with CMR had less focal myocardial scarring and functional impairment compared to patients diagnosed with standard assessment. CMR provided an accurate estimation of the extent of cardiac involvement, and may have reveal signs of early infiltration that were not detected by standard assessment. The extent of LGE related to severity of cardiac involvement and may therefore have prognostic implications

Chapter 5 evaluated the accuracy of gadolinium-enhanced CMR in the diagnosis of cardiac sarcoidosis. 
In 2005 the diagnosis of CS was made according to the guidelines of the Japanese Ministry of Health and Welfare (1993). CMR had not been incorporated in the guidelines, and the diagnostic accuracy of CMR for the diagnosis of CS had not yet been evaluated. We performed an analysis of the 12-lead ECGs, 24-hours ambulatory

ECGs, echocardiograms, thallium scintigrams and gadolinium-enhanced CMR studies in 58 biopsy proven PS patients assessed for CS. The diagnostic accuracy of CMR for CS was determined with modified Japanese guidelines as gold standard. Twelve of the 58 patients were diagnosed with CS (21\%). The sensitivity and specificity of CMR were $100 \%$, and $78 \%$, the PPV and NPV 55\% respectively $100 \%$, with an overall accuracy of $83 \%$. The combination of ECG and CMR had a sensitivity and specificity of $100 \%$ and $96 \%$, and PPV and NPV of respectively $86 \%$, and $100 \%$, with an overall accuracy of $97 \%$.

Late gadolinium enhancement (LGE) accurately delineates myocardial necrosis or focal fibrosis. The pattern of LGE in ischemic and non-ischemic myocardial disease is different, and might be helpful in distinguishing CS from ischemic disease. The pattern of LGE in CS has been reported to be patchy, multi-segmental, not related to coronary artery territories, while predominantly involving the mid myocardial and subepicardial layers. In Chapter 6 we reviewed the value of delayed contrast-enhanced CMR (DECMR) in differentiating patients with CS from those with coronary artery disease and recent myocardial infarctions. The DECMR studies of 30 patients with CS were compared to those performed in 30 consecutive infarct patients, who had been managed with primary coronary interventions, and 10 healthy controls. Two experienced blinded observers classified patients by assessing the distribution of LGE. Gadolinium CMR was helpful in differentiating patients with CS from patients with ischemic heart disease and previous myocardial infarctions. In a subgroup of ischemic patients the pattern of LGE was atypical, and suggestive of nonischemic etiology. Cardiac involvement in sarcoidosis is reported in up to $30 \%$ of patients. Left ventricular involvement demonstrated by DECMR has been well validated. We sought to determine the prevalence and distribution of RV LGE in patients diagnosed with PS. In Chapter 7 Right ventricular involvement in CS demonstrated with CMR. We prospectively evaluated 87 patients diagnosed with PS with contrast-enhanced cardiac magnetic resonance for RV involvement. Right ventricular enhancement was present in $16 \%$ of patients diagnosed with PS, and in $48 \%$ of patients with LV enhancement. The presence of RV enhancement correlated with pulmonary arterial hypertension, RV systolic dysfunction, hypertrophy and dilation. More extensive LV enhancement correlated with RV involvement. Right ventricular enhancement may result from direct infiltration and resulting scar, or pulmonary hypertension. Previous studies associated impaired systolic RV function and RV enhancement with ventricular tachy-arrhythmias. We demonstrate RV enhancement with cardiac magnetic resonance to be mostly multi-focal, involve the septum and correlate with increased RV volumes, hypertrophy and impaired systolic function. Finally in Chapter 8 we present the long-term follow up of 84 consecutive biopsy proven pulmonary sarcoidosis patients after baseline DECMR. Biventricular LGE at presentation was the strongest, independent predictor of adverse outcome during long-term follow up. Small asymptomatic LV myocardial scar of $<8 \%$ of LV mass carried a favorable long-term outcome. RV delayed enhancement correlates with systolic RV dysfunction and predicts ventricular tachy-arrhythmias as well as all cause death during follow up. This is the first pro- 
spective study to detail the prognostic relevance of right ventricular involvement in pulmonary sarcoidosis, and reports on improved long-term outcomes when cardiac sarcoidosis is managed according to current guidelines. 



\section{Chapter 11}

\section{Valorisation}

Cardiac sarcoidosis (CS) is a relatively rare but potentially devastating condition, which predominantly affects productive, middle-aged individuals. The results of the studies summarized in this thesis have helped improve the diagnosis and management of this condition. Knowledge valorization stands for the translation of academic wisdom to societal benefit. This can potentially be achieved by a broad range of products and activities. Our work made use of commercially available (imaging) technology, hardware and (postprocessing) software. Its aim was to demonstrate the exciting benefits of available imaging technology, Delayed-Enhanced Cardiovascular Magnetic Resonance (DECMR), and improve patient outcomes i.e. to prevent sudden cardiac death and promote quality of life. The practical application of our work was translated into the current Guidelines. (1-7) Findings with DECMR have been included as a major criterium in international, diagnostic and management, guidelines for CS. DECMR has been demonstrated to be a valuable diagnostic tool in sarcoidosis patients with cardiovascular symptoms and, as second line imaging technology, in patients with electrocardiographic or Doppler-echocardiographic abnormalities. Ongoing long-term prospective studies, which make use of the latest advances in CMR, may increase the success of targeted arrhythmia ablation, and result in more cost-effective implantation of cardioverter defibrillators.

The words of professor OP Sharma uttered in 1994 still ring true. "Myocardial sarcoidosis is difficult to diagnose, follows a treacherous course that may lead to death, and reponds poorly and randomly to treatment." "To deal successfully with the menace of myocardial sarcoidosis ....., one must first learn to think of the entity ....". "Once the presence of the wolf is suspected, further diagnostic studies should be aggressively pursued to establish the extent and severity of the illness." (8)

Knowledge about this condition, its insidious, non-specific nature, which mimics a number of alternative systemic conditions, needs to be actively promoted amongst all medical disciplines. The threshold for cardiac referral and evaluation should be low and any delays in the diagnostic process avoided. All available platforms, analogue (brochures, lectures, workshops) and digital (websites, podcast, ebook), should be recruited, targeting healthcare workers, professional societies, medical insurers, and the greater public. 


\section{References}

1. Birnie DH, Sauer WH, Bogun F, Cooper JM, Culver DA, Duvernoy CS, Judson MA, Kron J, Mehta D, Cosedis Nielsen J, Patel AR, Ohe T, Raatikainen P, Soejima K. HRS Expert consensus statement on the diagnosis and management of arrhythmias associated with cardiac sarcoidosis. Heart Rhythm 2014;11(7):13051323. doi: 10.1016/j.hrthm.2014.03.043.

2. Revised Guidelines for Diagnosis Cardiac Sarcoidosis 2006 (Japanese Society of Sarcoidosis and Other Granulomatous Disorders) criteria Japanese Ministery of Health 2006. Japanese Ministery Health Welfare. Diagnostic standard and guidelines for sarcoidosis. Jpn J Sarcoidosis Granulomatous Disord (Jpn) 2007;27:89-102.

3. Ishida Y, Yoshinaga K, Miyagawa M, Moroi M, Kondoh C, Kiso K, Kumita S. Recommendations for 18Ffluodeoxyglucose positron emission tomography imaging for cardiac sarcoidosis: Japanese Society of Nuclear Cardiology Recommendations. Ann Nucl Med 2014;28;393-403. doi: 10.1007/s12149-014-0806-0.

4. Costabel U, Skowasch D, Pabst S, Störk S, Schöpe C, Allewelt M, Worth H, Müller-Quernheim J, Grohé C. Diagnostik and Therapie der kardialen Sarkoidose. Pneumatologie 2014;68:124-132. doi: 10.1055/s-00331359197.

5. A joint procedural position statement on imaging in cardiac sarcoidosis: from the Cardiovascular and Inflammation \& Infection Committees of the European Association of Nuclear Medicine, the European Association of Cardiovascular Imaging, and the American Society of Nuclear Cardiology. Eur Heart J CVImaging 2017;18:1073-1089. doi:10.1093/ehjcvi/jex146

6. Terasaki F, Yoshinaga K. New guidelines for diagnosis of cardiac sarcoidosis in Japan. Ann Nucl Cardiol 2017;3:42-45. doi: 10.17996/anc.17-00042.

7. Slart RHJA, Glaudemans AWJM, Lancellotti P, Hyafil F, Blankstein R, Schwartz RG, Jaber WA, Russell R, Gimelli A, Rouzet F, Hacker, Gheysens O, Plein S, Miller EJ, Dorbala S, Donal E. A joint procedural position statement on imaging in cardiac sarcoidosis: From the Cardiovascular and Inflammation \& Infection Committees of the European Association of Nuclear Medicine, the European Association of Cardiovascular Imaging, and the American Society of Nuclear Cardiology. J Nucl Cardiol 2018;25(1):298-319. doi: 10.1007/s12350-017-1043-4.

8. OP Sharma. Myocardial sarcoidosis. A wolf in sheep's clothing. Chest 1994;106(4):988-990. doi: $10.1378 /$ chest106.4.988 


\section{Chapter 12}

\section{Acknowledgements}

During the twenty years between being introduced to and receiving formal training in cardiovascular magnetic resonance, I met many individuals, who either in some small but significant, or major conceptual and practical way contributed to the inception, development and delivery of the project detailed in this dissertation. All these helpful, selfless, hospitable, knowledgeable, and inspiring collegues, friends, relatives and mentors enriched my prolonged journey. This group consists of engineers, physicists, radiographers, radiologists, pulmonologists, technicians, nuclear physicians, and, inevitably, cardiologists.

Professor Harry Crijns - Dear Harry, you welcomed me in your department, supervised my projects, proved always available with advice, new insights and direction. I admire you as a leader, manager, enthousiast, unfatiqueable worker, a gentle Giant, who remained patient with me and supported my efforts after all those years. The $10000 \mathrm{kms}$ between Cape Town and Maastricht didn't dampen the spirit. I fondly remember dinner meetings with their unique Maastricht ambiance, grand rounds, and presentations by world class visiting lecturers. Your Department reflects the young, dynamic, pioneering spirit and international composition of Maastricht University.

Professor Anton Gorgels, Dr Pim Dassen, Dr Miel Cheriex, Dr Sebastiaan Bekkers, MUMC - Dear Anton without your vision, expertise, enthousiasm and support my efforts would have never been successful. Pim, Miel and Bas, I am grateful to have worked with you during my years at the MUCC. I greatly benefited from your practical support, and the high standards you set in clinical practice.

Fond memories of our secretaries - Emmy Damhuis (MUCC), Miriam Habex (MUCC), Karin Bouloukos (BBH, Cape Town) - ja Karin, this time I'm really finished!

Emmy, Miriam and Karin - you were always willing to support and assist, create appointments and meetings out of thin air, going extra miles, regularly reminding me I should stick to my promises, helping me to keep my feet firmly rooted on Dutch and African soil.

Yvonne Vercauteren - beste Yvonne, dank je dat bereid was om je eigen werkzaamheden en vrije tyd op te offeren om de software problemen van een wild-vreemde bezoeker uit Zuid Afrika op te lossen. You went the extra mile!

Dr Marinus van Kroonenburgh, Department of Nuclear Medicine, MUCC - Dear Marinus, I continue to admire your expertise and enthousiasm for nuclear cardiac imaging. Our collaboration was vital to the sarcoidosis program. 
Department of Radiology, MUCC - Dr Gabriel Snoep, radiologist and Liesbeth Boymans, radiographer. Dear Gabriel and Liesbeth, I remember our fruitful collaboration clearly, as we aimed to restart the CMR service at the hospital. I am thankful of the patience and grace you displayed when I firmly pushed for more cardiovascular scan time in an all ready stretched clinical program.

Dr Pieter van Paassen, immunologist - Pieter I continue to admire your expertise, drive and sense of humor. Your contributions to the sarcoidosis program in Maastricht were crucial.

Athol Wijnhoven, Pie Medical Imaging, Maastricht. Dear Athol I remain imdebted to your company for providing us with the post-processing software which allowed us to analyse the CMR studies on our patients in great detail. Your contributions were critical to our project.

\section{Cape Town}

Dr Rene Truter, radiologist, professor Anton Doubell, professor Gillian Ainslie and professor Bongani Mayosi, who recently tragically passed away. You all played a unique role in my training, practically, clinically, academically, and imaging wise. Dear Gillian, you have made a difference in the lifes of countless sarcoidosis patients. Thank you for all your efforts when allowing me to evaluate your patients with CMR.

\section{Rotterdam}

Dr Robert-Jan van Geuns, cardiologist, Erasmus MC - beste Robert-Jan, jy introduceerde me by het CMR programma in het EMC, en continueerde de evaluatie, zorg en opvolg van kardiale sarcoidose patienten in het EMC, nadat ik mijn opleiding tot cardiololoog in het EMC had afgerond, en Rotterdam voor Maastricht verruild had. Ik ben je dankbaar voor je kennis, visie, toewijding en vruchtbare samenwerking.

Dr Dave van Kraaij and dr Jacqueline Buys - beste Dave en Jacqueline, jullie vriendschap, gastvrijheid, inspirerende en motiverende aansporingen en vermaningen, de sauvignon blanc, stokjes satay, bitterballen en chocolate croissants van bakkerij van de Weerdt hebben uiteindelijk geresulteerd in het beoogde! I hope to continue our friendship over years to come and welcome you in Cape Town once again.

Specilene and Rudolph Francis - ek waardeer julle aanhoudende ondersteuning, julle het my gedruk tot op die meet! Die boekie is nou eindelik klaar, nou drink ons ietsie saam ...

\section{The Belgian connection}

I will forever be grateful for and impressed by the expertise, efficiency, hospitality and support of my Belgian colleagues, dr Veerle Reenaers, and dr Robert Geukens Hasselt, Belgium dr Joris Ector (Leuven), professor Hein Heidbuchel (Leuven, Hasselt, Antwerp). Thank you for sharing your knowledge and patients with me!

Professor Dudley Pennell, London - dear Dudley, thank you for providing me with accredited CMR training at the Royal Brompton Hospital, I have many fond memories of your Unit - you did not just introduce me to CMR but also to British hospitality, and some early pioneers in the field of cardiovascular magnetic resonance. 
My gratitude to those collegues who shared their knowledge and patients with me in the Netherlands - Liselotte van Erven (LUMC, Leiden), dr Joop Schreurs (the Hague), professor Richard Hauer (UMC, Utrecht).

Dr Harald Kuehl, cardiologist, Aachen, Germany- dear Harald, thank you for your willingness to help me interpret DECMR images of our first Maastricht patients.

\section{The Amsterdam connection}

Professor Albert van Rossum, and dr Aernout Beek, VUMC. Dear Bert, thank you for introducing me to cardiovascular magnetic resonance in 1986, even as a volunteer guinea pig the introduction was exhilarating, eventually leading me to my future wife in Cape Town, and a PhD in cardiac sarcoidosis. Aernout, dank je voor je bereidwilligheid om CMR beelden te evalueren!

To the many tireless radiographers, ('radiologisch laboranten") at MUCC, Groote Schuur Hospital, Panorama and Constantiaberg Medi-Clinics, Netcare N1 City Hospital as well as those at City Park Hospital the current Christiaan Barnard Memorial Hospital.

The artists

Stella van Schalkwijk, Michael Wyeth, Cape Town/Johannesburg, and Geert-Jan Zonneveld, MUCC - dear Stella, Michael and Geert-Jan, thank you for your diligence and skill in providing me with the beautiful, didactic figures which have improved our manuscripts and chapters substantially. Stella ek glo rerig die Here gaan jou terugvat Kaap toe!

Dear Heinz Morkel, nuclear physician (Cape Town), thank you for providing us with state-of-the-art nuclear imaging of some of our sarcoidosis patients in Cape Town.

Andrea Palmans, Michiel Janssen, cardiac technologists, MUCC - dank julle voor jullie vriendelijkheid, geduld en grote mate van deskundigeid in de evaluatie van onze sarcoidose patienten, met echocardiografie of de evaluatie van ICDs/PM, jullie waren altijd beschikbaar!

Dr Marlies Wysenbeek, MUCC - Marlies, jou werkzaamheden voor wat betreft de lange termijn opvolg van onze sarcoidose patienten was van onschatbaar belang voor de hoofdstukken 7 en 8 . Ek skuld jou big time!

I am greatly imdebted to dr Alfred Musekiwa's (University of the Witwatersrand, Johannesburg), dr Patty Nelemans, PhD, (University of Maastricht), and dr Justin Harvey (Stellenbosch University) who provided me with expert statistical support.

Lat but not least: Elmarie, Joshua, and Maja - thank you for allowing me to withdraw from your uplifting, fun-filled company, and allowing me to spend time in offices, quiet corners, and hotel rooms, to read and write, reflect, ... or leave to visit Harry, Dave and Jacqueline in Maastricht. The season to catch up and make good has arrived, more uninterrupted weekend break-aways, humbling rounds of golf, and exhilarating rides on surfboards.

Solo Deo Gloria! 



\section{Chapter 13}

\section{Curriculum Vitae}

Jan-Peter Smedema was born on May 1st 1964 in Sneek, The Netherlands. He attended high school in Den Helder, and studied Medicine at the Free University in Amsterdam. After finishing his compulsory basic military training he enjoyed working in the Respiratory Unit, Department of Medicine, at the Central Military Hospital in Utrecht (1989-1990). His first clinical work experience as civilian was in Cardio-Thoracic Surgery (Prof L Eysman, 1990-1991) and the Amsterdam Department of Interventional Cardiology (Dr F Kiemeney,1991-1992) at the Onze Lieve Vrouwe Gasthuis. The doors of opportunity and clinical exposure beckoned in South Africa. Starting in the Cardiac Unit at Ga-Rankuwa Hospital, Medical University of South Africa, Pretoria (Prof P Magobo) and Baragwanath Hospital, Johannesburg (Prof P Sareli, 1992). In 1993 he transferred to Tygerberg Hospital, the training hospital of Stellenbosch University, where he finished his training as a specialist physician (FCP (SA), MMed (Int Med), 1998), and enrolled in the cardiology training program (Prof AF Doubell). Whilst working in the Cardiac Unit, he encountered his first patient with cardiac sarcoidosis (CS), an encounter which ignited a life-long fascination with this condition. He visited professor AC van Rossum (VUMC, Amsterdam), immersed himself in Cardiovascular Magnetic Resonance (CMR) and started a local CMR program in Cape Town. After marrying his sweetheart CMR radiographer (who taught him more than just the basics of CMR), he took up professor D Pennell's invitation of a CMR fellowship at the Royal Brompton (2000). Subsequently he finished his clinical training as a cardiologist at the Thorax Centre, Erasmus University Rotterdam (Prof ML Simoons, 2002), where he met dr Robert-Jan van Geuns. They combined efforts and studied CS with delayed enhanced CMR (DECMR) in Rotterdam. Their collaboration continued after Jan-Peter was appointed as consultant cardiologist at Maastricht University Medical Centre (2002). Under the tutelage of professors AP Gorgels and HJGM Crijns his preliminary work studying of the value of DECMR in the management of CS, expanded formally into a PhD project. In 2005 he was invited to return to Cape Town to provide cardiovascular imaging services, and be trained in interventional cardiology ( $\mathrm{dr}$ A Saaiman). He was appointed honoury consultant at the Department of Medicine, Groote Schuur Hospital, University of Cape Town, and studied cardiomyopathy and tuberculous heart disease with DECMR (professors BM Mayosi and G Ainslie, 2005-2006). Since 2005 he has practiced as an interventional cardiologist with Netcare in Cape Town. Jan-Peter developed an interest in stroke medicine, joined a local stroke team, and obtained the MSc (Stroke Medicine), through the European Stroke Organization/Danube University, Austria (2015). He is currently enrolled in the Sports Science/Exercise Medicine program at the Sport Science Institute of South 
Africa (MPhil, UCT), and hopes to qualify as a sports physician in 2020. Jan-Peter continues to reside in Cape Town, South Africa, and enjoys life with his wife Elmarie, and two children, Joshua (19) and Maja (15). In his spare time he runs, hikes, and attempts to improve his golfing skills under the tutelage of his son.

Awards: Best oral presentation, South African Heart Association meeting 2010. 


\section{Publications}

1. Smedema JP, Laarman GJ. Perifere vasculaire complicaties bij hart catheterisatie. Netherlands Heart Journal 1993;1:4-8.

2. Wyser CP, Walzl G, Smedema JP, Swart F, van Schalkwijk E, van de Wal B. Corticosteroids in the treatment of tuberculous pleurisy: a double-blind, placebo-controlled, randomized study. Chest 1996;110:333-338.

3. Smedema JP, Doubell A, Kalis N, Gie R. Congenital unilateral absense of a pulmonary artery (UAPA) as an isolated finding and UAPA with contralateral peripheral pulmonary artery stenosis. Cardiovascular Journal of Southern Africa 1996;86;5:258-261.

4. Smedema JP. Geisoleerde congenitale unilaterale afwezigheid van een pulmonale arterie. Nederlands Militair Geneeskundig Tijdschrift 1996;6:192-193.

5. Smedema JP, Weich HFH. Peripheral vascular complications of cardiac catheterisation. Cardiovascular Journal of Southern Africa 1997;87:86-90.

6. Smedema JP, Saaiman A. Carotid Stent-Assisted Angioplasty: results in the first 33 patients. Cardiovascular Journal of Southern Africa 1997;87:9-14.

7. Smedema J.P. Cardiac Disease - An Update: Magnetic Resonance Imaging of the cardiovascular system. CME, March 2000.

8. van Rossum AC, Wynschank, Wagener G, du Plooy J, Smedema JP, Brink AJ. Round table discussion: Magnetic Resonance Imaging in cardiology - part 1. Cardiovascular Journal of Southern Africa, 2000;11(suppl 4).

9. van Rossum AC, Wynschank S, Wagener G, du Plooy J, Smedema JP, Brink AJ. Round table discussion: Magnetic Resonance Imaging in cardiology - part 2. Cardiovascular Journal of Southern Africa 2000;11(suppl 5).

10. Smedema JP, Katjitae I, Reuter H, Louw V, Doubell AF. 12 lead electrocardiography in tuberculous pericarditis. Cardiovascular Journal of Southern Africa, 2001.

11. Smedema JP, Katjitae I, Reuter H, Doubell AF. Ewart's sign in tuberculous pericarditis. South African Medical Journal, 2000;90(11):114.

12. Louw VJ, Reuter H, Smedema JP, Katiyitae I, Burgess L, Doubell AF. Twodimensional echocardiographically guided pericardiocentesis: experience in 170 consecutive patients. Netherlands Heart Journal, 2002.

13. Smedema JP, Doubell AF. Cardiac sarcoidosis: case-report and review of the literature. Cardiovasc J South Africa 2000;11(suppl 4).

14. De Backer T, Smedema JP, Klautz R, Maat L. Cardiac Myxoma. Thoraxcentre Journal, 2000;12(4):97.

15. Smedema JP, Doubell AF. Imaging: Congenital coronary fistula. Netherlands Heart Journal, 2001. 
16. De Backer T, Smedema JP, Carlier S. Primary pulmonary hypertension. Biodrugs 2001. 17. Smedema JP, Zondervan P, Bresser P, ten Cate FJ, Doubell AF, Hoogsteden HG, Balk AGHH. An African woman with erythema nodosum, and ventricular tachycardias. Thorax Centre Journal, 2001.

18. Smedema JP, Zondervan P, Bresser P, ten Cate FJ, Doubell AF, Hoogsteden HG, Balk AGHH. Cardiac sarcoidosis: case-reports and review of the literature. Netherlands Heart Journal, 2002.

19. van Kimmenade R, Smedema JP, van Suylen RJ. Rheumatic carditis in an 18 year-old man. Heart 2003;89 (9):1066.

20. Diacon AH, van de Wal BW, Wyser C, Smedema JP, Bezuidenhout J, Bolliger CT, Walzl G. Diagnostic tools in tuberculous pleurisy: a direct comparative study. Eur Respir J. 2003;22(4):589-591.

21. Oosting M, Smedema JP, Bekkers SCAM. A 60 year-old male with dyspnoea. Netherlands Heart Journal. 2004;12(8):353-354.

22. Liem N, Smedema JP, Steffels M. Cardiac involvement in CMML. Netherlands Heart Journal. 2004;12(10):467-468.

23. Smedema JP, Kroonenburgh van MPG, Snoep G, Bekkers SCAM, Bekkers, Gorgels AP. The diagnostic value of PET in cardiac sarcoidosis. J Nuclear Med 2004;45(11):1975.

24. Smedema JP, van Kroonenburgh MJPG, Snoep G, Backes W, Gorgels AP. Cardiac sarcoidosis in a patient with hypertrophic cardiomyopathy demonstrated by Magnetic Resonance Imaging and SPECT Dual Isotope scintigraphy. Circulation 2004;110:e529-531.

25. Smedema JP, Paassen van P, Snoep G, Kroonenburgh van MJPG, Crijns HJGM, Cohen Tervaert JW. Cardiac involvement in Churg Strauss syndrome demonstrated by CMR. Clin Exper Rheum, 2004;22(Suppl 36);S75-S78.

26. Smedema JP, Winckels S, Snoep G, Bekkers SCAM, Vainer J, Crijns HJGM. Tropical Endomyocardial Fibrosis (Davies' disease): Case report demonstrating the role of magnetic resonance imaging. Intern J of Cardiovasc Imaging, 2004;20:517-222.

27. Smedema JP, van Geuns RJ, Balk AH. A 53-year old female with biventricular heart failure. Netherlands Heart J 2005;13:193.

28. Smedema JP, Snoep G, van Kroonenburgh MPG, Dijkman B, Gorgels AP, Crijns HJGM. Nieuwe diagnostische en therapeutische technieken verbeteren de prognose van cardiale sarcoidose. Dutch Medical Journal. 2005;149:1168-1173.

29. Smedema JP, Snoep G, van Kroonenburgh MJPG, van Geuns RJ, Dassen WRM, Gorgels AP, Crijns HJGM. Evaluation of the diagnostic accuracy of gadolinium CMR in cardiac sarcoidosis. J Am Coll Cardiol 2005;45(10):1683-1690.

30. Smedema JP, Snoep G, van Kroonenburgh MPG, van Geuns, RJ, Dassen WRM, Gorgels AP, Crijns, HJGM. Prevalence of cardiac involvement in sarcoidosis in The Neth- 
erlands: Findings in 19 patients presenting with symptoms of cardiac involvement and 82 patients screened for cardiac involvement. Chest 2005;128(1):30-35.

31. Smedema JP, Snoep G, van Kroonenburgh MPG, Gorgels APM, Crijns HJGM. The value of gadolinium enhanced magnetic resonance imaging in addition to standard assessment for cardiac involvement in patients with pulmonary sarcoidosis. Chest 2005;128(3):1623-1628.

32. Smedema JP, Truter R, de Klerk PA, Zaaiman L, White L, Doubell AF. Cardiac sarcoidosis evaluated with gadolinium-enhanced cardiovascular magnetic resonance and contrast-enhanced 64-slice computed tomography. Int J Cardiol 2006;112(2):261-263.

33. Smedema JP, Tadamura E. Previous studies on delayed enhanced MRI for the detection of cardiac sarcoidosis. AJR Am J Roentgenol. 2006;186(2):578; author reply 578-579.

34. Smedema JP, Snoep G, Eerens F. Left ventricular viability in a patient with heart failure due to left main stem stenosis predicted by gadolinium enhanced magnetic resonance but not by dobutamine stress echocardiography. Cardiovasc J of South Africa 2005;1-3.

35. Smedema JP, Reenaers V, Geukens R. A 60-year-old woman with cardiac sarcoidosis. 2006;92(5):688.

36. Alzand B, Smedema JP, Barenbrug P. Late complication after closure of VSR. Netherlands Heart Journal, 2004.

37. Meijers, BKI, Schalla S, Eerens F, Van Suylen RJ, Broers B, Cheriex EM, Smedema JP. Protein loosing enteropathy in association with constrictive pericarditis. Int J Cardiovasc Imaging 2006;22(3-4):389-392.

38. Smedema JP, Bekkers SCAM, Snoep G. Comprehensive assessment of left ventricular pseudoaneurysms with magnetic resonance imaging. Cardiovasc J of South Africa, 2006;17(1):24-26.

39. Smedema JP. Letter to the Editor. The correlation between late gadolinium enhanced (LGE) magnetic resonance (MR), assessed with inversion-recovery gradient echo (IRGRE). Eur J Heart Fail. 2006;8(3):331; author reply 330.

40. Smedema E, Mayosi BM, Smedema JP. Hydatid disease - the 'water lily' sign. S Afr Med J. 2006;96(10):1042.

41. Smedema JP, Bekkers S, Snoep G. Late complication after closure of a ventricular septal rupture. Cardiovasc J S Afr. 2006;17(4):200-201.

42. Smedema JP, Bekkers SC, Snoep G. Comprehensive assessment of left ventricular pseudo-aneurysms with magnetic resonance imaging. Cardiovasc J S Afr. 2006;17(3):130-4.

43. Smedema JP, Snoep G, Van Kroonenburgh M, Eerens F. Left ventricular viability in a patient with heart failure due to left main stem stenosis, predicted by SPECT and gadolinium-enhanced magnetic resonance but not by dobutamine stress echocardiography. Cardiovasc J S Afr. 2006;17(1):24-26. 
44. Smedema JP. The correlation between late gadolinium enhanced (LGE) magnetic resonance (MR), assessed with inversion-recovery gradient echo (IRGRE). Eur J Heart Fail. 2006;8(3):331; author reply 330 .

45. Meijers BK, Schalla S, Eerens F, Van Suylen RJ, Broers B, Cheriex EM, Smedema JP. Protein-losing enteropathy in association with constrictive pericarditis. Int $\mathrm{J}$ Cardiovasc Imaging. 2006;22(3-4):389-392.

46. Smedema JP, Freeman VP. Images in cardiovascular medicine: Transkei heart. Neth Heart J. 2007;15(11):390-391.

47. Smedema JP. Images in cardiovascular medicine: myocardial inflammation in viral perimyocarditis detected by tissue Doppler echocardiography and magnetic resonance imaging. Cardiovasc J Afr. 2007;18(4):238-240.

48. Smedema JP, Abrahams S, Louw VJ. Partial reversal of hemolysis-associated pulmonary arterial hypertension in response to oral administration of L-arginine in a patient with hereditary spherocytosis. Acta Haematol. 2007;118(3):167-168.

49. Smedema JP, Louw VJ. Pulmonary arterial hypertension after splenectomy for hereditary spherocytosis. Cardiovasc J Afr. 2007;18(2):84-89.

50. Smedema JP, White L, Klopper AJ. FDG-PET and MIBI-Tc SPECT as follow-up tools in a patient with cardiac sarcoidosis requiring a pacemaker. Cardiovasc J Afr. 2008;19(6):309-310.

51. Russell JB, Syed FF, Ntsekhe M, Mayosi BM, Moosa S, Tshifularo M, Smedema JP. Tuberculous effusive-constrictive pericarditis. Cardiovasc J Afr. 2008;19(4):200-201.

52. Syed FF, Aje A, Ntsekhe M, Mayosi BM, Moosa S, Tshifularo M, Smedema JP. Resolution of nodular myocardial tuberculosis demonstrated by contrast-enhanced magnetic resonance imaging. Cardiovasc J Afr. 2008;19(4):198-199.

53. Smedema JP, Müller GJ. Coronary spasm and thrombosis in a bodybuilder using a nutritional supplement containing synephrine, octopamine, tyramine and caffeine. S Afr Med J. 2008 May;98(5):372-373.

54. Smedema JP. Tissue Doppler imaging in cardiac sarcoidosis. Eur J Echocardiogr. 2008;9(4):579-80.

55. Smedema JP, Berlion AE, Morkel H. Myocardial infarcts in a patient with a single right sided coronary ostium and interarterial course of the circumflex artery: the role of multimodality imaging. Int J Cardiol. 2009;132(1):e42-44.

56. Smedema JP, James R. Tissue Doppler imaging and contrast-enhanced cardiac magnetic resonance in primary cardiac amyloidosis. Eur J Echocardiogr. 2009;10(8):984-986.

57. Smedema JP. Cardiac magnetic resonance predicts reversibility of cocaine-induced ventricular dysfunction. Cardiovasc J Afr. 2009;20(3):198-199. 
58. Sliwa K, Blauwet L, Tibazarwa K, Libhaber E, Smedema JP, Becker A, McMurray J, Yamac H, Labidi S, Struman I, Hilfiker-Kleiner D. Evaluation of bromocriptine in the treatment of acute severe peripartum cardiomyopathy: a proof-of-concept pilot study. Circulation. 2010;121(13):1465-1473.

59. Smedema JP, Freeman V, Brink J. Aneurysm of the left aortic sinus causing acute myocardial infarction. Ann Paed Cardiology 2011.

60. Smedema JP, Pistorius L, Brink J. Late-onset pulmonary arterial hypertension after arterial switch surgery at birth. SA Heart J 2016.

61. Smedema JP, van Geuns RJ, Truter R, Mayosi BM, Crijns HJGM. Contrast-Enhanced Cardiac Magnetic Resonance: Distinction between cardiac sarcoidosis and infarction scar. Sarc Vasc Diff Lung Dis 2017.

62. Smedema JP van Geuns RJ, Ainslie G, Ector J, Heidbuchel H, Crijns HJGM. Right ventricular involvement in cardiac sarcoidosis demonstrated with cardiac magnetic resonance. ESC Heart Failure 2017.

63. Smedema JP, van Geuns RJ, Ector J, Heidbuchel H, Ainslie G, Crijns HJGM. The extent of delayed left ventricular enhancement and right ventricular involvement with contrast-enhanced cardiac magnetic resonance in cardiac sarcoidosis predict adverse outcome. ESC Heart Failure 2018. 
